\title{
Leitfaden
}

\section{für die Vorlesungen über darstellende Geometrie}

\author{
Von
}

Dr. Reinhold Muller

Vriag von fredr Veweg \& Sohn Braunsehvelg 


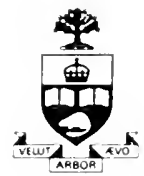

Presented to the

LIBRARY of the

UNIVERSITY OF TORONTO

from

the estate of

7.5. 


\section{.}


. 


\title{
Leitfaden
}

\section{für die Vorlesungen über}

darstellende Geometrie

\author{
Von \\ Dr. Reinhold Müller \\ Professor an der Technischen Hochschule zu Darmstadt
}

Dritte neubearbeitete und vermehrte Auflage

Mit 240 Abbildungen

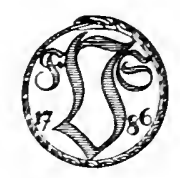

Braunschweig

Druck und Verlag von Friedr. Vieweg \& Sohn

1917 
Alle Rechte vorbehalten.

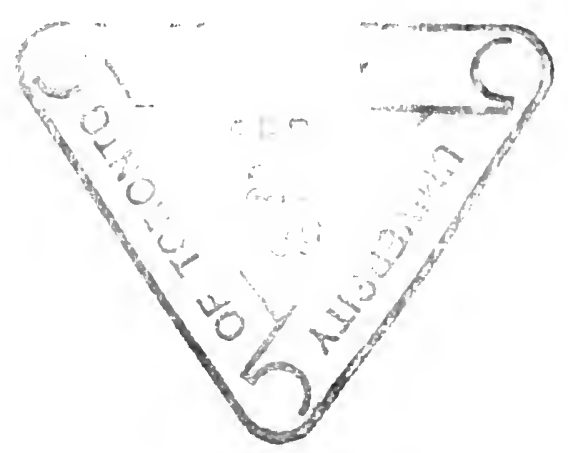

Copyriglit, 1917, by Friedr. Vieweg \& Sohn, Braunschweig, Germany. 


\section{Vorwort zur dritten Anflage.}

Die rorliegende dritte Auflage meines Leitfadens unter'scheidet sich von den vorhergehenden haułtsächlich durch eine stärkere Berücksichtigung der Anwendnngen. Einzelne Abschnitte, wie die über die topographischen Flächen und die Zentralprojektion, sind ganz neu bearbeitet worden; völlig unverändert sind nur kleine Teile des Buches geblieben. In theoretischer Hinsicht hat der behandelte Stoff keine wesentliche Vermehrung erfahren; namentlich babe ich anf die Heranziehung der projektiven Geometrie, der Photogrammetrie usw., die gesondert vorgetragen werdeu, nach wie vor verzichtet.

Wie bisher ist der Leitfaden in erster Linie für die Studierenden bestimmt, die gleichzeitig meine Vorlesungen hören. $\mathrm{Cm}$ aber seine Benutzung auch weiteren Kreisen zu erleichteru, sind auf Wunsch des Herrn Verlegers die Figuren stark vermehrt worden. Dabei habe ich mich zumeist auf die Angabe sorgfältig ausgewählter Daten beschränkt, um hierdurch den Leser zu zwingen, die Zeichnung aus den gegebenen Bestimmungsstücken - am besten in vergrößiertem Maßstabe - auf Grund des ausführlichen Textes eigenhändig zu entwerfen. Ich hoffe durch diese Anordnung namentlich solchen Studierenden zu nützen, die hereits einige Torkenntuisse in der darstellenden Geometrie besitzen, unter anderen auch denen, die das ku'z gefaßte Buch zur Vorbereitung auf eine Prüfung verwenden mörhten.

Darmstadt, in März 1917.

Der Verfasser. 


\section{Inhaltsverzeichnis.}

\section{Finleitung..................... . . . 1 \\ Erster Abschnitt.}

\section{Die Parallelprojektionen.}

I. Darstellung einfacher Raumgebilde in schiefer Parallelprojektion . 2

II. l'unkt, gerade Linie und Ebene in senkrechter Projektion auf zwei zueinander senkrechte Projektionsebenen. . . . . . . . 4

III. Hbenflächige Gebilde . . . . . . . . . . . . . . . . 29

IV. Der lireis . . . . . . . . . . . . . . . . . . 42

V. Die Kugel . . . . . . . . . . . . . . . . . . . 50

VI. Kegel- und Zylinderflächen . . . . . . . . . . . . . 54

VII. Umdrehungsflächen . . . . . . . . . . . . . . . . . . 86

VIII. Schraubenflächen . . . . . . . . . . . . . . . . 101

IX. Windschiefe Flächen . . . . . . . . . . . . . 116

X. Grundzüge der Belenchtungslehre . . . . . . . . . . . . . 121

XI. Kotierte Projektion und topographische Fläcben . . . . . . . . 123

તll. Axonometrie . . . . . . . . . . . . . . . 130

Zweiter Abschnitt.

Die Zentralprojektion.

I. Theoretische (freie) Perspektive . . . . . . . . . . . . 139

II. Angewandte Persjektive . . . . . . . . . . . . . 150

Anhang. Grundzüge der Reliefperspektive. . . . . . . . . . 177 


\section{Einleitung.}

Die darstellende Geometrie lehrt, räumliche Figuren in einer Ebene abzubilden und Aufgaben über diese Figuren auf Grundlage der Abbildung durch Zeichnung zu lösen.

Zur Herstellung solcher Abbildungen dient das Verfahren der Projektion: Um die Zentralprojektion einer gegebenen Figur zu erhalten, zieht man von einem festen Punkte (Auge oder Projektionszentrum) nach allen Punkten der Originalfigur gerade Linien (Sehstrahlen oder projizierende Strahlen) und bestimmt ihre Schnittpunkte mit der Bild- oder Projektionsebene. Rückt das Projektionszentrum in unendliche Entfernung, werden also die projizierenden Strahlen untereinander parallel, so entsteht eine Parallelprojektion, die als senkrecht (orthogonal) oder schief bezichuet wird, je nachdem die projizierenden Strahlen auf der Bildebene senkrecht stehen oder nicht.

An Stelle der Ebene kann unter Umständen eine krumme Fläche als Bildfläche treten; auch läßt sich das Projektionsverfabren in solcher Weise verallgemeinern, daß von den räumlicheu Objekten Bilder entstehen, die selbst wieder drei Dimensionen haben (Reliefperspektive, vgl. Anhang). 


\section{Erster Abschnitt. \\ Die Parallelprojektionen.}

\section{Darstellung einfacher Raumgebilde in schiefer Parallelprojektion.}

1. Ist $\Pi$ die Projektionsebene und $l$ eine Gerade, welche die Richtung der projizierenden Strahlen angibt, so erhält man von irgend einem Originalpunkte $P$ die Projektion $P_{s}$ als den Schnittpunkt von $\Pi$ mit der Parallelen durch $P$ zu 7 .

Konstruiert man zu allen Punkten $A, B, C \ldots$ einer Originalgeraden $g$, die nicht $\| l$ ist, die Projektionen $A_{s}, B_{s}, C_{s} \ldots$, so bilden die projizierenden Strahlen eiue Ebene (die projizierende Ebene von $g$ ),

Fig. 1.

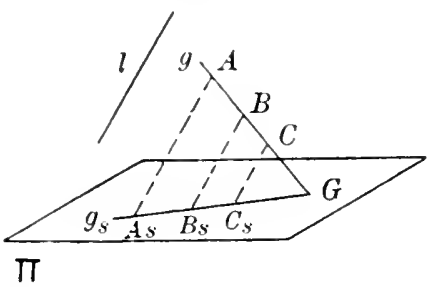

Fig. 2.

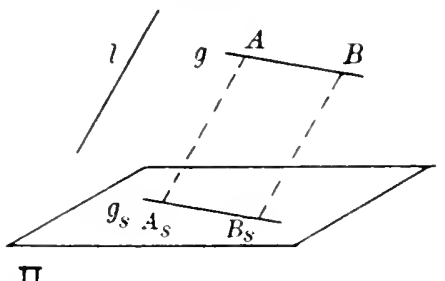

$\pi$

und diese schneidet die $\Pi$ in der Bildgeraden $g_{s}$ (Fig. 1). Die Projektion einer Geraden, die nicht die Riclitung der projizierenden Strahlen hat, ist also wieder eine Gerade; ist aber die Gerade || 1 , so ist ihre Projektion ein Punkt.

Die Gerade $g_{s}$ geht durch den Schnittpunkt $G$ von $g$ mit $\Pi$ (Spur oder Spurpunkt ron $g$ ).

Aus Fig. 1 folgt: Die Abschnitte auf der Bildgeradenverhalten sich wie die entsprechenden Abschnitte auf der Originalgeraden.

.Je nach der Wahl der Projektionsrichtung ist $A_{s} B_{s} \leq A B$. Ziehen wir aber in Fig. $2 g \| \Pi$, so wird $g_{s} \| g$, mithin $A_{s} B_{s}=A B$. Die

Fig. 3.

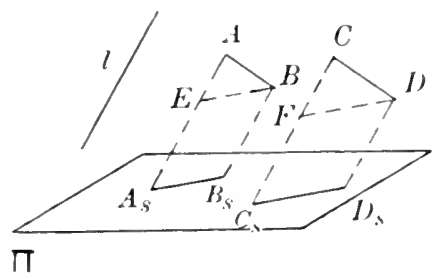

Projektion einer zur Bildebene parallelen Strecke ist also der Originalstrecke gleich und parallel. Ist demnach eine ebene Figur zur Bildebene parallel, so sind Bild-und Originalfigur kongruent und parallel.

2. Sind die Originalgeraden $A B$ und $C D$ parallel, so sind auch ihre 
projizierenden Ebenen parallel, mithin ist $A_{s} B_{s} \| C_{s}^{\prime} D_{s}$ (Fig. 3 ). D. h.: Parallelen Originalgeraden entsprechen parallele Bildgeraden.

Zieht man $B E \| B_{s} A_{s}$ bis $A A_{s}$ und $D F \| D_{s} C_{s}$ bis $C C_{s}$, so ergibt sich aus der Ähnlichkeit der Dreiecke $A B E$ und $C D F$ : Die Projektionen paralleler Strecken verhalten sich wie die Originalstrecken.

3. Die Fig. 1 bis 3 geben uns von der im Raume liegenden Bildebene $\Pi$ sowie von der Originalfigur und ihrer Projektion selbst wieder eine Abbildung - nämlich eine schiefe Parallelprojektion - auf die Zeichenebene. Indem wir jetzt dazu übergehen, einfache Körper in Parallelprojektion darzustellen, lassen wir die Bildebene $\Pi$ naturgemäß mit der Zeichenebene zusammenfallen. Dabei empfiehlt es sich, bezüglich der an sich vollkommen willkürlichen Richtung der projizierenden Strahlen ein Fig. 4. für allemal eine bestimmte Verabredung zu treffen; wir wollen etwa festsetzen: Diese Richtung soll immer so gewählt werden, daß die Projektion jeder auf der Zeichenebene $\Pi$

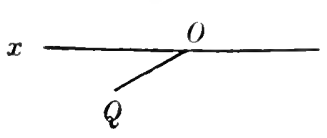
senkrechten Strecke halb so groß wird wie die Originalstrecke und mit der Breitenrichtung der Zeichenebene einen Winkel von $30^{\circ}$ einschließt. Oder genauer ausgedrückt: Ziehen wir in der vertikal gedachten Ebene $\pi$ durch irgend einen Punkt $O$ die horizontale Gerade $x$ sowie die beliebig lange Strecke $O Q$ unter einem Winkel von $30^{\circ}$ gegen $x$ und errichten in $O$ zu $\Pi$ nach vorn das Lot $O P=2 . O Q$, so soll die im Raume liegende Gerade $P Q$ die Richtung der projizierenden Strahlen angeben (Fig. 4).

Unter dieser Voraussetzung stellt in Fig. 1 bis 3 das mit $\Pi$ bezeichnete Parallelogramm ein horizontales Rechteck dar, von dem zwei Seiten zur Zeichenebene parallel sind. Man zeichne ebenso die schiefe Projektion eines Würfels mit horizontaler Grundfläche und zwei zu $\Pi$ parallelen Seitenflächen, ferner die Projektion eines aufrecht stehenden Kreuzes, dessen vordere Fläche $\| \Pi$ ist, dann die einer Treppe, deren Stufen auf $\Pi$ senkrecht stehen, usw.

4. Die schiefe Projektion eines Vielflachs ist ohne weiteres konstruierbar, wenn eine Reihe von Strecken bekannt ist, die zur Bildebene parallel oder senkrecht sind, und die das Vielflach in der angenommenen Lage bestimmen.

Darstellung einer regelmäligen fünfseitigen Pyramide mit horizontaler Grundfláche. Die vor $\Pi$ befindliche Grundfläche $A B C D E$ sei gegeben durch die Schnittlinie $x$ ihrer Ebene mit $\Pi$ und durch die Lage $A_{0} B_{0} C_{0} D_{0} E_{0}$, in die sie gelangt, wenn sie um $x$ nach unten in $\Pi$ umgelegt wird; dabei möge $C_{0} D_{0} \| x$ sein. Die Höhe der Pyramide sei $=h$ (Fig. 5 ).

Um zunächst die schiefe Projektion $A_{s}$ von $A$ zu ermitteln, ziehe man $A_{0} J \perp x$ als Umlegung des ron $A$ auf $x$ gefällten Lotes, sowie $J A_{s}$ unter $30^{\circ}$ gegen $x$. Nacht man dann $J A_{s}=\frac{1}{2} J A_{0}$ (bequemer zieht man $A_{0} A_{s}$ unter $60^{\circ}$ gegen $x$ ), so ist $J A_{s}$ die Projektion von 
$J A$. - Auf $A_{0} J$ liegen die Mittelpunkte $F_{0}, G_{0}$ von $B_{0} E_{0}, C_{0} D_{0}$, sowie der Mittelpunkt $M_{0}$ des Fünfecks. Zieht man durch diese Punkte Paralleleu zu $A_{0} A_{s}$, so findet man anf $J A_{s}$ die entsprechenden Punkte $F_{s}, G_{s}, M_{s}$. Wa $B E \| x$, also $\| \Pi$ ist, so ist auch $B_{s} L_{s} \| x$ und

Fig. 5.

\section{$x$}

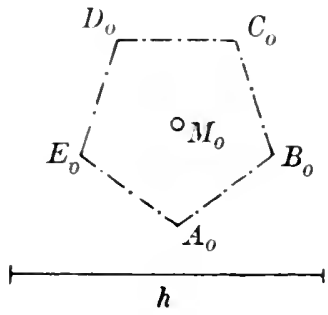

$F_{s} B_{s}=F_{s} E_{s}=F_{0} B_{0}$. Ebenso ergibt sich $C_{s}^{\prime} D_{s}$. Die Hölenlinie der Pyramide ist $\| \Pi$ und $\perp x$; ihre I'rojektion geht also durch $M_{s} \perp x$ und ist $=h$.

5. Projiziert man die bisher dargestellten Körper in unveränderter Lage senkrecht auf $\Pi$, so zeigt sich, daß die entstehenden Bilder weniger anschaulich sind als die zuvor erlialtenen, weil alle Geraden und Ebenen, die auf $\Pi$ senkrecht stehen, bzw. als Punkte und Geraden abgebildet werden. $\mathrm{Um}$ auch in senkrechter Projektion anschauliche Bilder zu erhalten, mübte man die besondere Lage aufgeben, welche die Körper gegen die Bildebene einnehmen, was jedoch die Konstruktion der Bilder erheblich erschweren würde. Die schiefe Projektion erweist sich demnach als vorzugsweise geeignet, um von stereometrischen Figuren anschauliche skizzen zu zeichnen, und in diesem Sinne werden wir sie im folgenden immer benutzen.

\section{Punkt, gerade Linie und Ebene in senkrechter Projektion auf zwei zueiuander senkrechte Projektionsebenen.}

Darstellung des Punktes.

6. Ein Punkt im Raume ist durch Angabe seiner schiefen oder senkrechten Projektion noch nicht bestimmt. Das gebräuchlichste Verfabren, um die Lage des Originalpunktes zu bestimmen, besteht in der Anwendung senkrechter Projektion anf zwei zueinander senkrechte Projektionsebenen, von denen die eine immer horizontal, die andere also vertikal gestellt wird. Indem wir diese Darstellungsweise den folgenden Entwickelungen zugrunde legen, lassen wir die vertikale Projektionsebene stets mit der Zeichenebene (Wandtafel) zusammenfallen und gebrauchen die folgenden Bezeichnungen:

Horizontal-oder Grundrißebene, erste Projektionsebene, e rste Tafel, $\Pi_{1}$;

Vertikal- oder Anfrißebene, zweite Projektionsebene, zweite Tafel, $\Pi_{2}$;

Projektionsachse oder kurz Achse für die Schnittlinie $x$ beider Ebenen;

ferner in bezug auf einen Originalpunkt $P$ :

Horizontalprojektion oder Grundriß, erste Projektion, $P^{\prime}$; Vertikalprojektion oder Aufrib, zweite Projektiou, $P^{\prime \prime}$; erster bzw. " weiter projizierender Strahl $P I^{\prime \prime}, P P^{\prime \prime}$. 
Wir unterscheiden vordere und hintere $\Pi_{1}\left(+\Pi_{1}\right.$ und $\left.-\Pi_{1}\right)$, sowie obere und untere $\Pi_{2}\left(+\Pi_{2}\right.$ und $\left.-\Pi_{2}\right)$. Die Projektionsebenen teilen den Raum in vier $Q u a d r a n t e n$, die in der Reihenfolge $\left(+\Pi_{1},+\Pi_{2}\right),\left(-\Pi_{1},+\Pi_{2}\right),\left(-\Pi_{1},-\Pi_{2}\right),\left(+\Pi_{1},-\Pi_{2}\right)$ als erster bis vierter Quadrant bezeichnet werden.

Bei der Herstellung von Grund - und Aufriß denken wir uns das unendlich ferne Auge oberhalb der $\Pi_{1}$ bzw. vor der $\Pi_{2}$ und die Projektionsebenen als undurchsichtig; deshall, wird der abzubildende Gegenstand nur dann von beiden Augen gesehen, wenn er sich im ersten Quarranten befindet, und wir legen ihn daher in Zukunft, wenn möglich, immer in diesen Quadranten.

Fig. 6 zeigt rechteckig begrenzte Teile der beiden Projektionsebenen und einen im ersten Quadranten liegenden Punkt $Y$ mit seinen

Fig. 6.

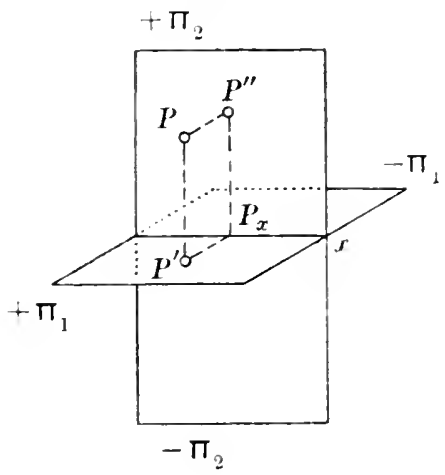

Fir. 6 a.

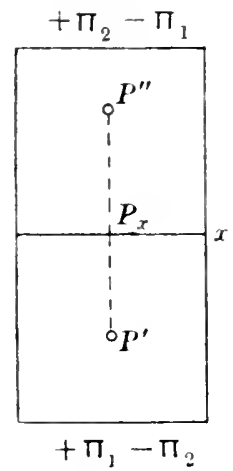

projizierenden Strahlen, dargestellt in schiefer Projektion unter der Voraussetzung, daß die $\Pi_{2}$ mit der Zeichenebene zusammenfällt, oder zu ihr parallel ist; der Index $s$, den wir bisher zur Bezeichnung der schiefen Projektion benutzt hatten, ist hier der Einfachheit wegen weggelassen.

7. Die Ebene $P P^{\prime} P^{\prime \prime}$ ist senkrecht auf $\Pi_{1}$ und $\Pi_{2}$, also auch auf $x$; verstelien wir demnach unter $P_{x}$ ihren Schnittpunkt mit $x$, so sind $P^{\prime} P_{x}$ und $P^{\prime \prime} P_{x}$ auf $x$ senkrecht, d. h.: Die von den beiden Projektionen eines Punktes a $f$ die Achse gefällten Lote treffen sich in der Achse.

In dem Rechteck $P P^{\prime} P_{x} P^{\prime \prime}$ ist $P P^{\prime}=P^{\prime \prime} P_{x}$; in Worten: Die Entfernung eines Punktes von der ersten Projektionsebene (sein erster Tafelabstand) ist gleich der Entfernung seiner zweiten Projektion von der Achse. Ebenso ist $P P^{\prime \prime}=P^{\prime} P_{x}$.

8. Um die Zeichnung auf eine einzige Ebene zu beschränken, drehen wir die $\Pi_{1}$ um $x$, bis sie mit $\Pi_{2}$ zusammenfällt, und zwar wollen wir diese Umlegung immer in der Weise ausführen, daß die $+\Pi_{1}$ auf die $-\Pi_{2}$ zu liegen kommt. Dann fällt $P^{\prime} P_{x}$ in die Gerade $P^{\prime \prime} P_{x}$, d. h.: Die beiden Projektionen eines Punktes liegen in einem Lote zur Projektionsachse (Fig. 6a). 
L'mgekehrt bilden irgend zwei Punkte $P^{\prime}$ und $P^{\prime \prime}$ der Zeichenebene, deren Verbindungslinie auf $x$ senkrecht steht, die beiden Projektionen eines Raumpunktes $P$, der auf diese Weise eindeutig bestimmt ist.

Fig. 7 stellt einen Punkt $Q$ des zweiten Quadranten dar. Man zeichne ehenso die Projektionen eines Punktes des dritten sowie des

Fig. 7.

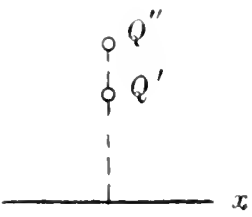
vierten Quadranten. Nur bei den Puukten des ersten Quadranten liegt derGrundribunterhalb $x$, der Aufrib oberhalb $x$.

Liegt ein Punkt in der ersten Projektionsebene, so liegt seine $\mathrm{zweite}$ Projektion in der Achse; befindet sich der Punkt in der zweiten Projektionsebene, so liegt seine erste Projektion in der Achse.

Wir nennen Halbierungsebenen die beiden durch die Achse gehenden Ebenen $\mathrm{H}_{1}$ und $\mathrm{H}_{2}$, die den ersten und dritten bzw. den zweiten und vierten Quadranten halbieren. Die beiden Projektionen eines Punktes der $H_{1}$ liegen symmetrisch zu $x$, diejenigen eines Punktes der $\mathrm{H}_{2}$ fallen zusammen.

9. Obwohl ein Raumgebilde durch Grund- und Aufrib seiner Punkte bereits vollkommen bestimmt ist, erweist es sich unter Umständen als

Fig. 8.

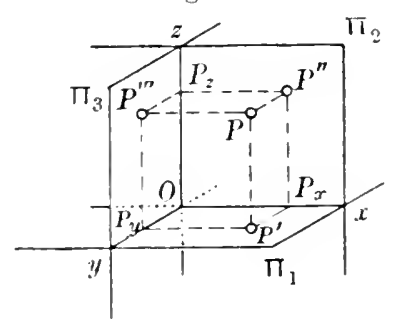
zweckmäßig, seine senkrechte Projektion auf eine dritte Ebene $\Pi_{3}$ hinzuzufügen, die auf $\Pi_{1}$ und $\Pi_{2}$, also auf $x$ senkrecht ist und Seitenriß-oder Kreuzrißebene genannt wird. Dann entstehen die neuen Projektionsachsen $y=\Pi_{1} \times \Pi_{3}$ und $z=\Pi_{2} \times \Pi_{3}$, und die drei Geraden $x, \eta, z$ schneiden sich rechtwinklig im Punkte $O=\Pi_{1} \times \Pi_{2} \times \Pi_{3}$. Fig. 8 gibt hiervon eine Skizze in schiefer Parallelprojektion. Ist $P^{\prime \prime \prime}$ die dritte Projektion des Punktes $P$, so haben die von $P^{\prime}$ und $P^{\prime \prime \prime}$ auf $y$ gefällten Lote denselben Fußpunkt $P_{y}$ (Art. 7). Ebenso treffen sich die Lote von $P^{\prime \prime}$ und $P^{\prime \prime \prime}$ auf $z$ in einem Punkte $P_{z}$.

Fig. 8 a.

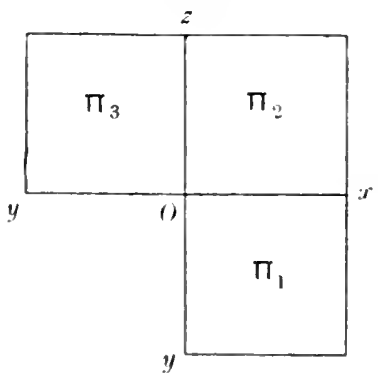

Fig. 8 b.

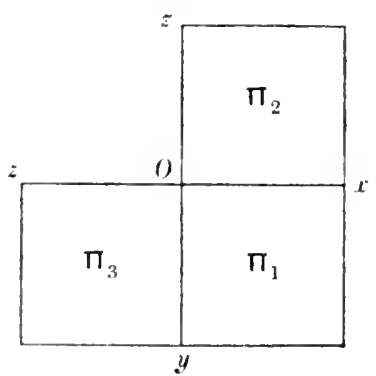

Die drei Tafelabstände $P^{\prime \prime \prime} P=O P_{x}, P^{\prime \prime} P=O P_{y}, P^{\prime} P=O P_{z}$ werden in der analytischen Geometrie des Raumes als die Koordinaten 
von $P$ bezeichnet. Sie dienen zur eindeutigen Bestimmung von ${ }^{\prime}$, wenn jeder einzelne Abstand von der betreffenden Ebene aus nach der einen Seite positiv, nach der entgegengesetzten negativ gerechnet wird.

Zum Zwecke der Darstellung wird naturgemäls auch die $\Pi_{3}$ mit der Zeichenebene $\Pi_{2}$ zur Deckung grebracht. Man erreicht dies am einfachsten durch eine Drehuug um $z$, etwa so, daß die vordere $\Pi_{3}$ auf die linke Halbebene $\Pi_{2}$ zu liegen kommt (Fig. 8a). Man kann aber auch die $\Pi_{3}$ zunächst um $y$ in die $\Pi_{1}$ umlegen, z. B. die obere $\Pi_{3}$ in die linke Halbebene $\Pi_{1}$, und dann die vereinigten Ebenen um $x$ in dem früher festgesetzten Sinne so lange drehen, bis sie mit $\Pi_{2}$ zusammenfallen (Fig. 8b). MIan beachte, daß bei der ersten Art der Umlegung die $y$-Achse, bei der zweiten die $z$-Aclise in der Figur doppelt anftritt.

10. Grundaufgabe. Aus der ersten und zweiten Projektion $P^{\prime}$ und $P^{\prime \prime}$ eines Punktes $P$ die dritte $P^{\prime \prime \prime}$ zu konstruieren (Fig. 9). Die Ebene $\Pi_{3}$ sei durch die Gerade $z \perp x$ gegeben.

Fig. 9.

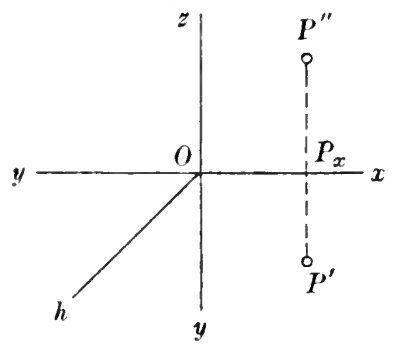

Fig. 10.

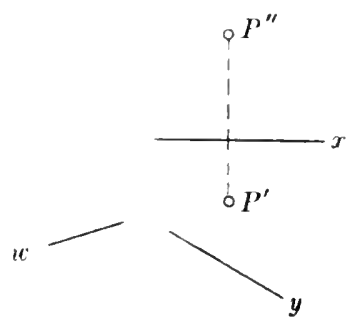

Legt man sie wie in Fig. $8 \mathrm{a}$ in die Ebene $\Pi_{2}$ um, so fällt die Strecke $P_{z} P^{\prime \prime \prime}$, die $=P_{x} P^{\prime}$ ist, in die Verlängerung von $P^{\prime \prime} P_{2}$. Hieraus folgt für die Bestimmung von $P^{\prime \prime \prime}$ die einfache Regel: Nan ziehe durch $P^{\prime \prime}$ eine Parallele zu $x$ und mache aufihr von $z$ aus die Strecke $P_{s} P^{\prime \prime \prime}=P_{x} P^{\prime}$. - Ohne Benutzung des Zirkels erhält man $P^{\prime \prime \prime}$ mittels der Geraden $h$, die den rechten Winkel zwischen den beiden Umlegungen desselben Teiles der $y$-Achse halbiert, durch $P^{\prime} P_{h} \| x$ bis $h$ und $P_{h} P^{\prime \prime \prime} z$.

Bei der in Fig. $8 \mathrm{~b}$ angedeuteten Art der Umlegung ziehe man die Gerade $P^{\prime} P_{y} \| x$ bis $y$ und mache auf ihrer Terlängerung die Strecke $P_{y} P^{\prime \prime \prime}=P_{x} P^{\prime \prime}$.

11. Zuweilen ist es vorteilhaft, die neue Projektionsebene so zu wählen, daß sie nur auf einer der beiden ursprünglichen Tafeln senkrecht steht. Sei z. B. $\Pi_{3} \perp \Pi_{1}$ und $y$ die Schnittlinie beider Ebenen (Fig. 10). Legt man, wie bei der zweiten Lösung der vorhergehenden Aufgabe, die $\Pi_{3}$ um $y$ in die $\Pi_{1}$ um, so ist nach Art. $8 P^{\prime} P^{\prime \prime \prime} \perp y$ und nach Art. $7 P^{\prime \prime \prime} P_{y}=$ Abstand $P \Pi_{1}=P^{\prime \prime} P_{x}$. In Worten: Der A bstand der neuen Projektiou von der neuen Achse ist gleich dem Abstande der wegfallenden Projektion von der alten Achse.

12. In derselben Weise findet man weiter die Projektion $P^{1 V}$ von $P$ auf eine vierte Tafel $\Pi_{4}$, die auf der $\Pi_{3}$ senkrecht steht. Dann bildet die Schnittlinie $v$ der beiden Tafeln, die in Fig. 10 durch ihre 
Umlegung in die $\Pi_{1}$ gegeben ist, eine neue Projektionsachse. Denkt man sich also die $\Pi_{4}$ um $w$ in die $\Pi_{3}$ und mit.dieser in die $\Pi_{1}$ umgeklappt, so ist $J^{\prime \prime \prime} P^{\prime \prime} \perp u$ und Abstand $I^{\prime \prime} w=$ Abstand $I^{\prime} y$.

Die Einführung neuer Projektionsebenen kann dazu dienen, um von einem Gegenstande anschauliche Bilder zu erhalten, wenu die ursprünglichen Projektionen wegen der besonderen Lage des Gegenstandes gegen die Projektionsebenen zu wenig anschaulich sind.

Man verfahre so bei einem Würfel, dessen Grundfläche $A B C D$ in $\Pi_{1}$ liegt, und dessen Kante $A B \| x$ ist.

13. Ist ein Gegenstand durcl seine Projektionen $A^{\prime} B^{\prime} C^{\prime} \ldots$ und $A^{\prime \prime} B^{\prime \prime} C^{\prime \prime} \ldots$ gegeben, und verschiebt man die Projektionsachse $x$ parallel $z u$ sich selbst um die Strecke $e$ z. B. nach unten, so vermebrt man die ersten Tafelabstinde aller Punkte $A, B, C \ldots$ um $e$ und rermindert ihre zweiten 'Tafelabstande um denselben Betrag. Dann sind aber' $A^{\prime} B^{\prime} C^{\prime} \ldots$ und $A^{\prime \prime} B^{\prime \prime} C^{\prime \prime} \ldots$ die Projektionen, die sich von dem dargestellten Gegenstande ergeben, wemn man ihn $\perp \Pi_{1}$ nach ohen und $\perp \Pi_{2}$ nach hinten jedesmal um $e$, also im ganzen um die Strecke e $1 \overline{2} \perp \mathrm{H}_{1}$ verschiebt.

Läbt man daher die Projektionsachse ganz weg, so ist durch die beiden Projektionen der Gegenstand selbst bestimmt, seine Lage gegen die Projektionsebenen aber nur bis auf Schiebungen senkrecht zur Halbierungsebene $\mathrm{H}_{1}$.

Nun kommt es bei technischen Zeichnungen auf die Lage des Gegenstandes gegen die Projektionsebenen nicht an, und deshalb pflegt man bei solchen Zeichnungen auf die Angabeder Projektionsachse überhaupt zu verzichten.

Um dann von dem durch seine Projektionen $A^{\prime} B^{\prime} C^{\prime} \ldots$ und $A^{\prime} B^{\prime \prime} C^{\prime \prime} \ldots$ gegebenen Gegenstande eine dritte Projektion auf eine $\Pi_{3} \perp \Pi_{1}$ zu koustruieren, darf man z. B. $A^{\prime \prime \prime}$ beliebig wählen. Zieht man durch $A^{\prime \prime}$ die Gerade $u \perp A^{\prime} A^{\prime \prime}$ und durch $A^{\prime \prime}$ die Gerade $\imath \perp A^{\prime} A^{\prime \prime \prime}$, so ergibt sich $B^{\prime \prime \prime}$ aus der Bedingung

$$
\text { Abstand } B^{\prime \prime \prime} v=\text { Abstand } B^{\prime \prime} u \text {. }
$$

\section{Darstellung der Geraden.}

14. Eine Originalgerade $q$, die auf keiner der Projektionsebenen senkrecht steht, hat zu ihrem Grund- und Aufrib zwei Geraden $g^{\prime}$ und $g^{\prime \prime}$, nämlich die Schnittlinien ihrer ersten und zweiten projizierenden Ebene bzw. mit $\Pi_{1}$ und $\Pi_{2}$ (vgl. die skizze 11 in schiefer Parallelprojektion und die entsprechende Fig. 1la in Grund- und Aufriß).

Ist $J^{\prime}$ ein Punkt von $g$, so liegt $l^{\prime}$ auf $g^{\prime}, P^{\prime \prime}$ auf $g^{\prime \prime}$, und zwar so, daf $P^{\prime} P^{\prime \prime} \perp x$ ist. Daher verhalten sich die Abschnitte auf $g^{\prime}$ wie die entsprechenden Abschnitte auf $g^{\prime \prime}$.

15. Gerade Linien in besonderer Lage gegen die Projektionsebenen.

a) Ist $y \| \Pi_{1}$, so ist $g^{\prime \prime} \| x$ (und $g^{\prime} \| g$ ). - Ist $g \| \Pi_{2}$, so ist $g^{\prime} \| x$ (und $g^{\prime \prime} \|(y)$.

b) Ist $g \| x$, so sind $g^{\prime}$ und $g^{\prime \prime} \| x$. 
c) Ist $g \perp \Pi_{1}$, so ist $g^{\prime}$ ein P'unkt und $g^{\prime \prime} \perp x$. - Ist $g \perp \Pi_{2}$, so ist $g^{\prime} \perp x, g^{\prime \prime}$ ein Punkt.

d) Ist $g \perp x$, d. h. liegt $g$ in einer zu $x$ senkrechten Ebene, so hefiuden sich $q^{\prime}$ und $g^{\prime \prime}$ in einer zu $r$ senkrechten Geraden.

16. Zwei beliebige Geraden $g^{\prime}$ und $g^{\prime \prime}$ der Zeichenebene, von denen keine auf $x$ senkrecht steht, können umgekehrt als Projektionen einer Geraden (I im Raume angesehen werden, die a uf diese Weise eindeutig bestimmt ist. Bringt man nämlicb die $\Pi_{1}$ mit der in ihr liegenden Geraden $g^{\prime}$ in ihre ursprüngliche Lage $\perp \Pi_{2}$, so ergibt sich $g$ als Schnittlinie der beiden projizierenden Ebenen, die durch $g^{\prime}$ und $g^{\prime \prime}$ bzw. $\perp \Pi_{1}$ und $\perp \Pi_{2}$ gelegt werden.

Stelit aber $g^{\prime} \perp x$, so ist $g^{\prime \prime}$ entweder ein Punkt, oder $g^{\prime}$ und $g^{\prime \prime}$ liegen in derselben Senkrechten zu $x$. Im letzten Falle decken sich die beiden projizierenden Ebenen; die Originalgerade ist also durch Angabe von $g^{\prime}$ und $g^{\prime \prime}$ noch nicht bestimmt. Hierzu bedarf es der Projektionen $A^{\prime}$, $A^{\prime \prime}$ und $B^{\prime}, B^{\prime \prime}$ zweier Punkte $A$ und $B$ (Fig. 12). Um dann zu einem dritten Punkte $C$ der Geraden, der durch $C^{\prime}$ bestimmt ist, die zweite Projektion $C^{\prime \prime} z u$ ermitteln, lege man die projizierende Ebene von $g$ um $g^{\prime \prime}$ in die $\Pi_{2}$ um, oder man ermittle die dritte Projektion $g^{\prime \prime \prime}=1^{\prime \prime \prime} B^{\prime \prime \prime}$ auf eine $\Pi_{3} \perp x$.

Fig. 11.

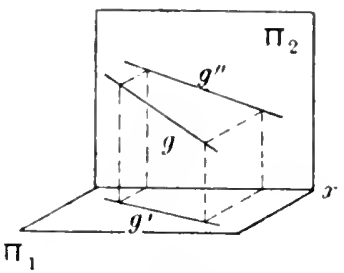

Fig. $11 \mathrm{a}$.

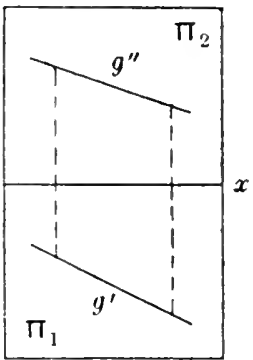

17. Unter dem ersten bzw. zweiten Spurpunkte der Geraden $g$ versteht man ihre Schnittpunkte $G_{1}$ und $G_{2}$ mit $\Pi_{1}$ und $\Pi_{2}$ (Art. 1 ).

Grundaufgabe. Die Spurpunkte der durch ihre Projektionen $g^{\prime}, g^{\prime \prime}$ gegebenen Geraden $g$ zu konstruieren (Fig. 13).

Fig. 12.

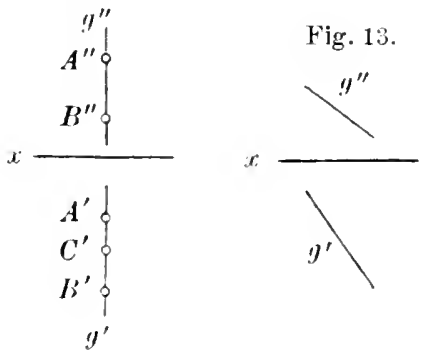

Fig. 14.
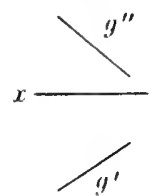

Fig. 15.

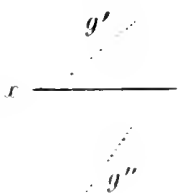

Da der Punkt $G_{1}$ der Geraden $g$ angehört, so liegt $G_{1}^{\prime}$ in $g^{\prime}$ und $G_{1}^{\prime \prime}$ in $g^{\prime \prime}$ : dabei ist $G_{1}^{\prime} G_{1}^{\prime \prime} \perp x$ (Art. 14). Da sich ferner $G_{1}$ in $\Pi_{1}$ befindet, so fällt $G_{1}^{\prime}$ mit $G_{1}$ zusammen, und $G_{1}^{\prime \prime}$ liegt in $x$. Nan bestimme demnach den Schnittpunkt $G_{1}^{\prime \prime}$ von $g^{\prime \prime}$ mit $x$ und ziehe $G_{1}^{\prime \prime} G_{1} \perp x$ bis $g^{\prime}$. In analoger Weise ergibt sich $G_{2}$ aus $G_{2}^{\prime}=g^{\prime} \times x, G_{2}^{\prime} G_{2} \perp x$ bis $g^{\prime \prime}$. 
Nach Ermittelung der Spurpunkte erkennt man, welcher Teil der Geraden $g$ im ersten Quadranten liegt; die entsprechenden Teile von $g^{\prime}$ und $g^{\prime \prime}$ sind als sichtbar auszuziehen (Art. 6). Der im zweiten Quadranten befindliche T'eil von $g$ wirl zwar vom ersten projizierenden Auge gesehen, aber der zugehörige 'Teil von g' liegt auf der $-\Pi_{1}$, und diese wird beim UTmlegren ron der $+\Pi_{2}$ verdeckt. Ebenso ist der im vierten Quadranten liegende Teil von $g$ für das zweite projizierende Auge sichtbar, aber der entsprechende 'leil ron $g$ " wirl durch die umgelegte $+\Pi_{1}$ verleckt. - In Fig. 13 liegt $G_{1}$ unterhalb $x$ und deshalb in der $+\Pi_{1}, G_{2}$ oberhalb $x$, mithin in der $+\Pi_{2}$; von der gegebenen Geradeu befindet sich also die Strecke $G_{1} G_{2}$ im ersten Quadranten.

Man bestimme auch bei den in Figr. 14 und 15 dargestellten Goraden die Spurpunkte und den im ersten Quadranten liegenden Teil. Diesar Teil hat seinen Grundrib stets gauz unterhalb $x$ und seinen Aufrif oberhalb $x$ (Art. 8). In Fig. 15 tritt also die Gerade $g$ überhaupt nicht in den ersten Quadranten ein.

Liegt die Gerade, wie in Fig. 12, in einer auf $x$ senkrechten Ebene, so erhält man inre Spurpunkte durch Umlegung dieser Ebene in die $\Pi_{2}$ oder mittels eines Seitenrisses (Art. 16).

18. Grundaufgabe. Die Neigungswinkel $\gamma_{1}, \gamma_{2}$ zu ermitteln, welche die durch ibre Spurpunkte $G_{1} . G_{2}$ gegebene Gerade $g$ bzw. mit $\Pi_{1}, \Pi_{2}$ bildet. (Erste und zweite Tafel-

Fig. 16.

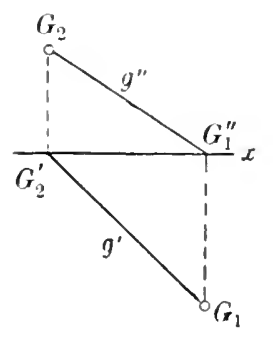

Fig. 16 a.

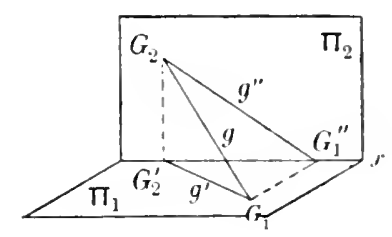

neigung von $y$, Fig. 16 mit der Skizze 16a in schiefer Parallelprojektion.) Der Winkel $\gamma_{1}$ liegt im Raume bei $G_{1}$ in dem rechtwinkligen Dreieck $G_{2} G_{1} G_{2}^{\prime}$, dessen Katheten $G_{2} G_{2}^{\prime}$ und $G_{2}^{\prime} G_{1}^{\prime}$ bekannt siud; macht man also auf $x$ die Strecke $G_{2}^{\prime} \dot{r}_{1}^{0}=G_{2}^{\prime} G_{1}^{\prime}$, so ist $\angle G_{2} G_{1}^{\prime \prime} G_{2}^{\prime}=\gamma_{1}$ und $\Delta G_{2} G_{1}^{0} G_{2}^{\prime}$ die Umlegung ron $\Delta G_{2} G_{1} G_{2}^{\prime}$ in die $\Pi_{2}$. Ebenso erhält man $\gamma_{2}$ durch Imlegung von $\Delta G_{1} G_{2} G_{1}^{\prime \prime}$ in die $\Pi_{1}$.

Unter allen Winkeln, welche $g$ mit den durch $G_{2}$ gehenden Geraden ron $\Pi_{2}$ einschliebt, ist bekanntlich $\gamma_{2}$ der kleinste, also $\gamma_{2} \leqq \angle G_{1} G_{2} G_{2}^{\prime}$, wobei das Gleichbeitszeichen sich auf den Ausnabmefall $g \perp x$ beziebt. Nun ist aber $\angle G_{1} G_{2} G_{2}^{\prime}=90^{\circ}-\gamma_{1}$, folglich

$$
\gamma_{1}+\gamma_{2} \leqq 90^{\circ}
$$

Bezeichnet man mit $7, l^{\prime}, l^{\prime \prime}$ bzw. die Längen einer auf $y$ liegenden Originalstrecke, sowie ihrer ersten und zweiten Projektion, so ist

$$
l^{\prime}=7 \cos \gamma_{1}, \quad l^{\prime \prime}=l \cos \gamma_{2} \text {. }
$$


Ist also die Strecke $l \mathrm{zu}$ keiner Projektionsebene parallel, so erscheint sie in jeder Projektion verkürzt, und $z$ war sind $\cos \gamma_{1}$ und $\cos \gamma_{2}$ die Verkürzungsverhältnisse.

19. Grundaufgabe. Die wahre Längederdurch ihre Projektionen $A^{\prime} B^{\prime}, A^{\prime \prime} B^{\prime \prime}$ gegebenen Strecke $A B$ zu konstruieren (Fig. 17 und Skizze 17 a in schiefer Parallelprojektion). Zieht man in der ersteu projizierenden Ebene von $A B$ die Gerade $B C \| B^{\prime} A^{\prime}$ bis $A A^{\prime}$, so entstelit das rechtwinklige Dreieck ill $C$ mit den Katheten $C B=A^{\prime} B^{\prime}$ und $A C=A A^{\prime}-B B^{\prime}=A^{\prime \prime} A_{x}-B^{\prime \prime} B_{x}$. Die wahre Länge von $A B$ ist also gleich der Hypotenuse eines rechtwiukligen

Fig. 17.

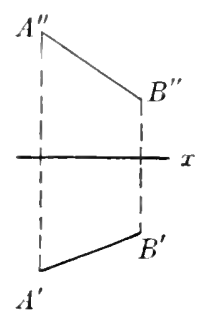

Fig. 17 a.

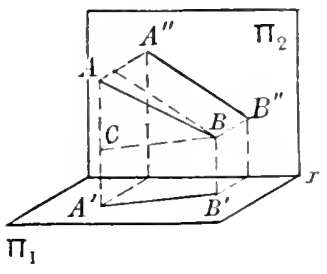

Dreiecks, welches $A^{\prime} B^{\prime}$ und $A^{\prime \prime} A_{x}-B^{\prime \prime} B_{x}$ zu Katheten hat. Man ziehe daher die Gerade $B^{\prime \prime} C^{\prime \prime} \mid x$ bis $A^{\prime \prime} A^{\prime}$ und mache auf ihr $C^{\prime \prime} B_{0}^{\prime \prime}$ $=A^{\prime} B^{\prime} ;$ dann ist $A^{\prime \prime} B_{0}^{\prime \prime}=A B$.

Die ebeu ausgeführte Konstruktion läßt sich auch so deuten: Das Dreieck $A B C$ ist durch Drehung um seine vertikale Kathete $A C$ in eine neue Lage $A B_{0} C$ gebracht worden, in der es $\| \Pi_{2}$ ist. Dann erscheint es im Aufriß in wahrer Größe.

Ebenso ergibt sich $A B$ als Hypotenuse eines rechtwinkligen Dreiecks mit den Katheten $A^{\prime \prime} B^{\prime}$ und $A A^{\prime \prime}-B B^{\prime \prime}=A^{\prime} A_{x}-B^{\prime} B_{x}$. Überhaupt gilt also der Satz: Die wahre Länge der Strecke $A B$ ist die Hypotenuse eines rechtwinkligen Drejecks, das die eine Projektion von $A B$ und die Differenz der Abstände der Punkte $A$ und $B$ von der betreffendeu Projektionsebene zu Katheten hat.

20. Zwei Geraden liegen entweder in einer Ebene - und danu schneiden sie sich in einem endlichen oder einem unendlich fernen Punktel) (parallele Geraden) - oder sie sind windschief zueinander.

1) Man sagt bekanntlich, jede Gerade $g$ besitze einen unendlich fernen Punkt, weil durch einen auBerhalb liegenden Punkt $P$ nur eine Parallele zu $g$ gezogen werden kann, und weil auch jede andere Gerade, die in der Ebene $P g$ durch $P$ gelegt wird, einen einzigen Punkt mit $g$ gemein hat. Der unendlich ferne Punkt einer Geraden ist identisch mit dem, was man sonst ihre Richtung nemnt. Ebenso wird jeder Ebene eine unendlich ferne Gerade zugeschrieben, nämlich der Ort der unendlich fernen Punkte aller in ihr liegenden Geraden. Zwei parallele Ebenen haben dieselbe unendlich ferne Gerade (Stellung). - Unter der unendlich fernen Ebene des Raumes versteht man den Inbegriff aller unendlich fernen Punkte und Geraden. 
Wenn zwei Geraden einander schneiden, so liegen die Schnittpunkte der gleichnamigen Projektionen in einem Lot zur. Achse. Der umgekehrte Satz gilt nur daun nicht unbedingt, wenn eine der beiden Geraden $\perp x$ ist.

Sind $g^{\prime}, q^{\prime \prime}$ und $h^{\prime}, h^{\prime \prime}$ die Projektionen zweier windschiefen Geraden $g$ und $h$, so ist der Schnittpunkt von $g^{\prime}$ und $h^{\prime}$ der Grundrib eines Punktes al von ! und eines Punktes $B$ von $h$. Liegr, wie in

Fig. 18.

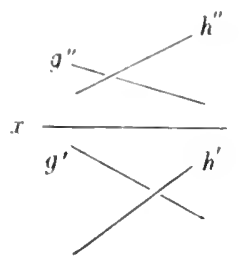

Fig. is, $B^{\prime \prime}$ höher als $A^{\prime \prime}$, so liegt $B$ über $A$, und die Gerade $h^{\prime}$ überdeckt $!^{\prime}$ in $A^{\prime}$. Ebenso entsprechen dem schnittpunkte von $g^{\prime \prime}$ und $l^{\prime \prime}$ auf $g^{\prime}$ und $l^{\prime}$ bzw. zwei Punkte $C^{\prime}$ und $D^{\prime}$. Liegt dann $C^{\prime}$ näher an $x$ als $I^{\prime}$, so liegt $l$ ) vor $C$, und $l^{\prime \prime}$ überdeckt $l^{\prime \prime}$ in $C^{\prime \prime}$. Sind zwei Geraden einander parallel, so sind die gleichuamigen Projektionen parallel (Art. "2). - Sind umgekehrt die Geraden $g$ und $h$ durch ihre Projektionen $g^{\prime}, l^{\prime \prime}$ und $l^{\prime}, h^{\prime \prime}$ gegeben, und ist $g^{\prime}\left\|h^{\prime}, g^{\prime \prime}\right\| h^{\prime \prime}$, so sind die gleichnamigen projizierenden Ebenen parallel, folgtich ist auch $g \| h$. In dem Ausnahmefalle, daß $g^{\prime}$ und $g^{\prime \prime}$, sowie $h^{\prime}$ und $h^{\prime \prime}$ in je einer Senkrechten zu $x$ liegen, hat man $g^{\prime \prime \prime}$ und $h^{\prime \prime \prime}$ zu konstruieren, um zu entscheiden, ob $y \| l$ ist.

\section{Darstellung der Ebene.}

21. Eine Ebene $E$ ist bestimmt durch die Projektionen von drei ihrer Punkte $A, B, C$ oder von zwei ihrer Geraden $g, h$. Von der

Fig. 19 a.

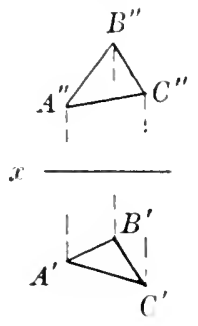

Fig. $19 \mathrm{~b}$.

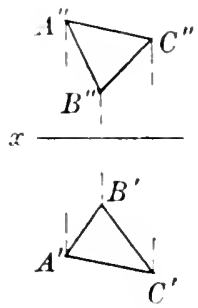

Fig. 20.

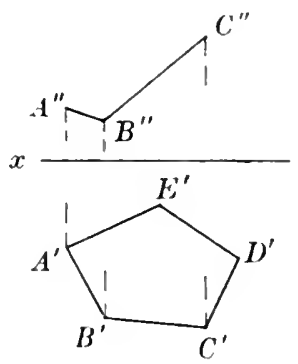

Ebene $A B C$ sieht man im Grund- und Aufrif dieselbe Seite oder entgerrengesetzte Seiten, je nachdem die Dreiecke $A^{\prime} B^{\prime} C^{\prime \prime}$ und $A^{\prime \prime} B^{\prime \prime} C^{\prime \prime}$ gleichen oder entgegengesetzten Sinnes sind (Fig 19 a und b).

Kennt man von einem ebenen Vieleck $A B C D \ldots$ den Grundriß $A^{\prime} B^{\prime} C^{\prime} D^{\prime} \ldots$ und von drei Punkten $A, B, C$ auch den Aufriß $A^{\prime \prime}, B^{\prime \prime}, C^{\prime \prime}$, so sind die zweiten Projektionen der übrigen Eckpunkte bestimmt (Fig. 20). Nan findet z. B. zum Punkte $J^{\prime}=A^{\prime} C^{\prime} \times I^{\prime} I^{\prime}$ den Punkt $J^{\prime \prime}$ auf $I^{\prime \prime} C^{\prime \prime}$, somit die Gerade $I^{\prime \prime} J^{\prime \prime}$ und auf dieser den Punkt $D^{\prime \prime}$.

22. Häufig benutzt man zur Bestimmung der Ebene E ihre Spurlinien $e_{1}$ und $e_{2}$, d. h. ihre Schnittlinien mit $\Pi_{1}$ und $\Pi_{2} ; e_{1}$ und $e_{2}$ treffen sich auf $x$ in Achsenschnitpunkte $E_{x}$. Zwischen der Bestimmung einer Ebene durch Spuren und der Bestimmung durch zwei boliebige Geraden besteht aber kein wesentlicher Unterschied; es ist nur 
$e_{1}^{\prime}=e_{1}, e_{1}^{\prime \prime}=x$ und $e_{2}^{\prime}=x, e_{2}^{\prime \prime}=e_{2}$. Bilden die sichtbaren 'Teile von $e_{1}$ und $e_{2}$ mit derselben $R$ chtung von $x$ spitze Winkel, so sieht man im Grund- und Aufrils dieselbe Seite der Ebene (Fig.21 a und b).

Aufgabe. Die dritte Spurlinie $e_{3}$ der Ebene $E\left(e_{1}, e_{2}\right) z u$ konstruieren (Fig. 22 und Skizze 22a in schiefer Parallelprojektion).

Fig. 22.

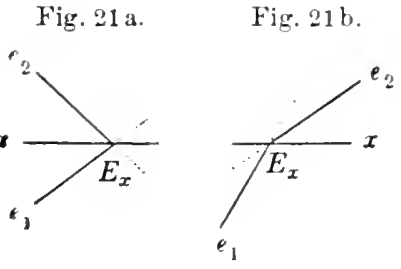

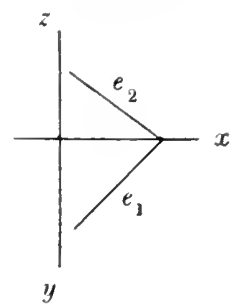

Fig. 22 a

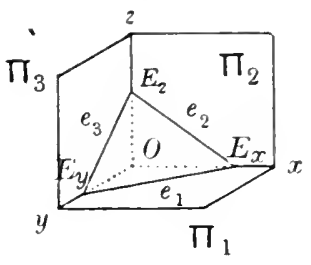

Man bestimme die Punkte $E_{y}=e_{1}>y$ und $E_{z}=e_{2} \times z$, daun ist $e_{3}=E_{y} E_{z}$. Wird die $\Pi_{3}$ um $z$ in die $\Pi_{2}$ umgelegt, so gibt es in Fig. 22 zwei Lagen rou $F_{y}$.

Ebenen in besonderer Lage gegen die Projektionstafeln.

a) Ist $\mathbf{E} \| \Pi_{1}$, so ist $e_{2} \| r$ und $e_{1}$ unendlich fern. Jeder Punkt von $\mathrm{E}$ hat seinen Aufrif auf $e_{2}$. - Ist $\mathrm{E} \| \Pi_{2}$, so ist $e_{1} \| x$ und $\rho_{2}$ unendlich fern.

b) Ist $\mathrm{E} \perp \Pi_{1}$, so ist $e_{2} \perp x$, und der Grundrib jedes Punktes ron $E$ liegt auf $e_{1}$. Ist $\mathrm{E} \perp \Pi_{2}$, so ist $e_{1} \perp x$.

c) Ist $\mathrm{E} \| x$, so sind $e_{1}$ und $e_{2} \| x$.

23. Gerade Linien und Punkte in einer Ebene. Grundanfgabe. Von der Geraden $i$, die in der Ebene E liegt, ist gegeben $i$, gesucht $i^{\prime \prime}$. Ist $E$ durch zwei sich schneidende Geraden

Fig. 23.

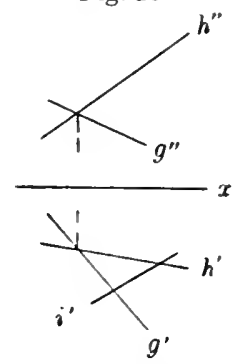

Fig. 24.

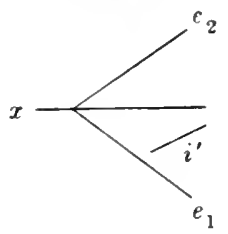

$g$ und $h$ gegeben, so kennt man von den Schnittpunkten $A$ und $B$ der Geraden $i$ mit $g$ und $h$ die Grundrisse $A^{\prime}$ und $B^{\prime}$; dann ist $i^{\prime \prime}=A^{\prime \prime} B^{\prime \prime}$ (Fig. 23).

Liegt eine Gerade in einer Ebene, so liegen die Spurpunkte der Geraden in den gleichnamigen Spurlinien der Ebene. Ist also die Ebene $\mathbf{E}$ durch ihre Spuren $e_{1}$ und $e_{2}$ bestimmt und die in ihr liegende Gerate $i$ durch $i$ gegeben, und soll wieder $i^{\prime \prime}$ konstruiert werden, so suche man zu den Schnittpunkten $J_{1}$ und $J_{2}^{\prime}$ von $i^{\prime}$ mit $e_{1}$ und $x$ die zweiten Projektionen $J_{1}^{\prime \prime}$ auf $x$ und $J_{2}$ auf $e_{2}$; dann ist $i^{\prime \prime}=J_{1}^{\prime \prime} J_{2}$ (Fig. 24). 
Grundaufgabe. Vom Pnnkte $P$ der Ebene E ist gegeben $P^{\prime}$, gesucht $P^{\prime \prime}$. Man ziehe durch $P^{\prime}$ irgend eine Gerade $i^{\prime}$ als Grundrib einer in $E$ liegenden Geraden $i$ nud ermittle wie vorhin $i^{\prime \prime}$; dann liegt $l^{\prime \prime}$ auf $i^{\prime \prime}$.

24. Anfgabe. DieSpurlinien der EbeneE zu konstruieren, die durch $z$ wei sich schneidendeGeraden $y$ und $h$ bestimmt ist (Fig. 25). Lösung: $e_{1}=\left(r_{1} H_{1}, e_{2}=\left(i_{2} I_{2}\right.\right.$. Lontrolle: $e_{1}$ und $e_{2}$ schneiden sich auf $x$. - Sind die Spurpunkte von $g$ und $h$ zum Teil oder sämtlich unerreichbar, so ziehe man in geeigneter Weise eine oder mehrere Hilfsgeraden, deren jede $g$ mol $h$ schneidet. Wählt man den

Fig. 27.

Fig. 25 .

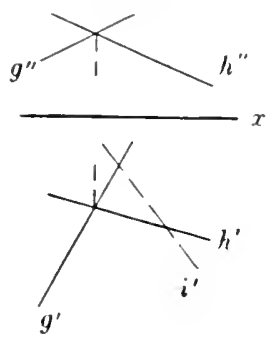

Fig. 26 a. Fig. 26 b.
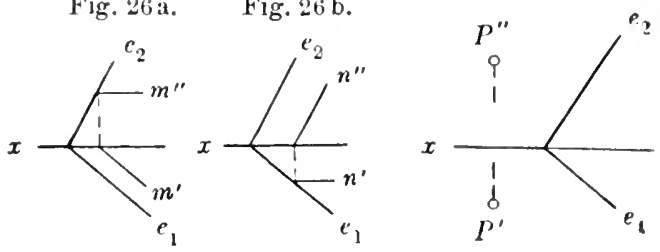

Grundric $i^{\prime}$ einer solchen Geraden beliebig, so findet man bieraus $i^{\prime \prime}$, und daun gehen $e_{1}$ und $e_{2}$ bzw. durch die Spurpunkte $J_{1}$ und $J_{2}$ von $i$. Aufgabe. Gegeben zweizueinander windschiefeGeraden $g$ und $h$; durch $g$ eine Ebene E zu legen $\| h$. Man ziehe durch einen beliebigen Punkt von $g$ die Gerade $l \| h$, so ist $E=g l$.

25. Unter Hauptlinien (Spurparallelen) einer Ebene versteht man die Geraden der Ebene, die zu einer Projektionsebene, also zur betreffenden Spurlinie parallel sind. Ist $m$ eine erste, $n$ eine $z$ weite Hauptlinie von $\mathrm{E}$, so ist $m^{\prime}\left\|e_{1}, m^{\prime \prime}\right\| x$ und $x^{\prime}\left\|x, n^{\prime \prime}\right\| c_{2}$ (Fig. $26 \mathrm{a} \mathrm{u.} \mathrm{b).}$

Durch Benutzung der Hauptlinien gelangt man zu neuen Lösungen der früheren Grundaufgabe (Art. 23): Von dem Punkte P, der in der Ebene $\mathrm{E}=e_{1} e_{2}$ liegt, ist gegeben $P^{\prime}$, gesucht $I^{\prime \prime}$.

26. Sind zwei Ebenen parallel, so sind diegleichnamigen Spuren parallel.

Aufgabe. Durch den Punkt $P$ eine Ebene $\Phi$ zu legen, die zur Ebene $E$ parallel ist. Zieht man durch $P$ eine Gerade $m$ parallel zu irgend einer Geraden von $E$, so geht $\Phi$ durch $m$. Ist $E$ durch die Spuren $e_{1}$ und $e_{2}$ gegeben, und sind diese nicht $\| x$, so legt man zweckmäbig $m \| e_{1}$ oder $\| e_{2}$; im ersten Falle geht die Spur $f_{2}$ von $\Phi$ durch den Spurpunkt $M_{2}$ von $m$ und ist $\| e_{2}$ (Fig. 27).

\section{Schnitte ron Ebenen und Geraden.}

27. Grundaufgabe. Die Schnittlinie $g$ zweier Ebenen $E$ und $\Phi$ zukonstruieren, die durchibre Spurlinien $e_{1}, e_{2}$ und $f_{1}, f_{2}$ bestimmt sind (Fig. 2s). Nach dem Satze in Art. 23 kennt man von $g$ die Spurpunkte $G_{1}=e_{1} \times f_{1}$ und $i_{2}=e_{2} \times f_{2}$. - Ist $G_{1}$ unerreichbar, 
so benutze man eine Hilfsebene $\Delta$ und konstruiere zunächst die Schnittlinien $h=\Delta \times \mathrm{E}$ und $i=\Delta \times \Phi$; damn geht $g$ durch den Schnittpunkt von $h$ mit $i$. Wählt man $\Delta \| \Pi_{1}$, so ist $h \| e_{1}$ und $i \| f_{1}$.

28. Grundaufgabe Den Schnittpunkt $P$ der Geraden $g$ mit der Ebene $\mathrm{E}=A B C \mathrm{zu}$ konstruieren (Fig. 29). IIan lege durch $g$ eine Hilfsebene, am zweckmäbigsten eine projizierende Ebene, z. B. die erste, und bestimme ihre Schnittlinie $i$ mit E; diese schneidet $g$ in $I^{\prime}$. Bekannt ist $i^{\prime}=g^{\prime}$; hieraus ergibt sich $i^{\prime \prime}$ and $P^{\prime \prime}=g^{\prime \prime} \times i^{\prime \prime}$. - Aus dem Aufriß erkennt man,

Fig. 29.

Fig. 28.

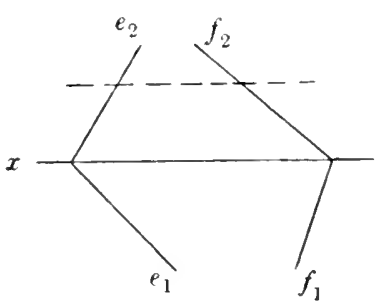

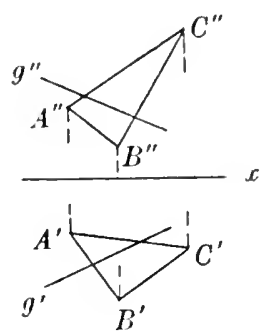

Fig. 30.

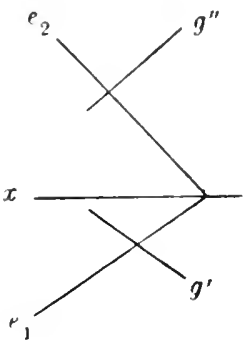

welcher Teil von $g$ unterhalb $i$ liegt, also in Grundriß von der undurchsichtigen Dreiecksfläche verdeckt wird. Oder man untersucht wie in Art. 20, ob die Seite $A^{\prime} B^{\prime}$ die Gerade $g^{\prime}$ überdeckt, oder ob sie umgekehrt von $g^{\prime}$ überdeckt wird. - Sind $A^{\prime} B^{\prime} C^{\prime}$ und $A^{\prime \prime} B^{\prime \prime} C^{\prime \prime}$ gleichen Sinnes, so sieht man in Grund- und Aufriß denselben Teil von $g$.

Ebenso findet man den Schnittpunkt von $g$ mit einer Ebene, die durch ibre Spuren gegeben ist (Fig. 30).

29. Grundaufgabe. Die Schnittlinie $g$ der Ebenen zweier Dreiecke $A B C$ und $D E F \quad$ zu konstruieren (Fig. 31). Man bestimme nach der vorigen Aufgabe z. B. die Schnittpunkte $J$ und $K$ der Geraden $D E$ und $E F$ mit der Ebene $A B C$, dann ist $g=J K$. In Fig. 31 liegt

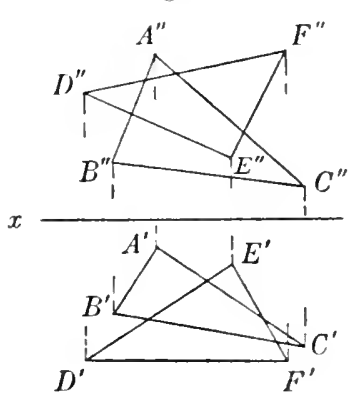
$K$ außerhalb des Dreiecks $A B C$, deshalb reicht die Schnittlinie der begrenzten Ebenen nur bis zum Schnittpunkte von $J K$ mit $A C$, den man natürlich auch als Schnitt ron $A C$ mit der Ebene $D E F$ hätte ermitteln können.

Man beachte, daß bei diesen wie bei den folgenden Aufgaben die Projektionsachse entbehrlich ist, falls die gegebenen Geraden und Ebenen nur durch Projektionen, nicht durch Spuren festgelegt siud (vgl. Art. 13).

Gerade Linien und Ebenen in rechtwinkliger Lage.

30. Ist ein Schenkel eines rechten Winkels zu einer Projektionsebene parallel, so ist die senkrechte Projektion des Winkels anf diese Ebene wieder ein rechter. Sind nämlich $g$ 
und $h$ die Schenkel eines rechten Winkels mit dem Scheitel $A$, und ist $h \| \Pi_{1}$, so steht $h$ senkreclit anf $A A^{\prime}$ und auf $g$, also auf der Ebene $A A^{\prime}, g$. Dann ist aber auch die zu $h$ parallele Gerade $h^{\prime}$ auf derselben Ebene senkrecht, also senkrecht auf der Geraden $g^{\prime}$ dieser Ebene.

Bezeichnet man mit $i$ irgend eine zu ! parallele Gerade im Raume, so ist auch $i^{\prime} \perp h^{\prime}$, d. h.: Sind überhaupt zwei (sich schneidende oder windschiefe) Geradensenkrecht a ufeinander, und ist die eine ron ihuen zu einer Projektionsebene parallel, so bilden ihre senkrechten Projektionen auf diese Ebene gleichfalls einen rechten Winkel. Ist umgekelurt $h^{\prime} \perp i^{\prime}$ und $h \| \Pi_{1}$, so ist $h \perp i$.

Steht die Gerade g senkrecht auf der Ebene E, so steht sie auch senkrecht auf deren Spuren $e_{1}$ und $e_{2}$. I ann ist aber nach dem zweiten

Fig. 83 .

Fig. 32.

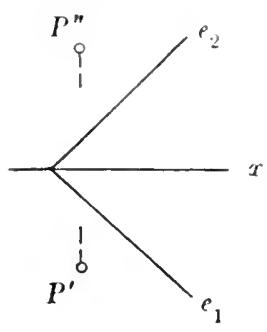

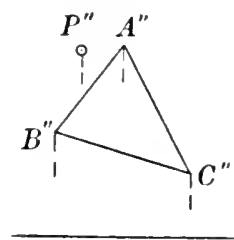

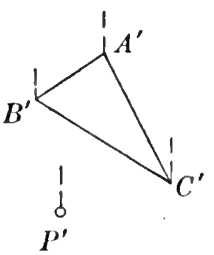

Fig. 34 .

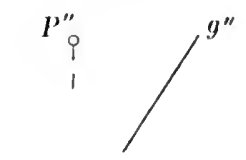

$x$

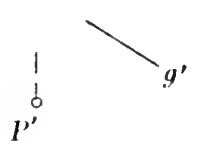

Satze $g^{\prime} \perp e_{1}$ und $g^{\prime \prime} \perp e_{2}$. Daraus folgt: Steht eine Gerade senkrecht auf einer Ebene, so stehendiesenkrechten Projektionen der Geraden senkrecht auf den gleichnamigen Spurlinien der Ebene. Umgekehrt folgt aus $g^{\prime} \perp e_{1}$ und $g^{\prime \prime} \perp e_{2}$ auch $g \perp \mathrm{E}$, falls nicht $\mathrm{E} \| x$ ist.

31. Anwendungen. Grundaufgabe. Vom Punkte $P$ auf die Ebene $\mathrm{E}\left(e_{1} e_{2}\right)$ ein Lot $l$ zu fällen (Fig. 32) Man ziehe durch $P^{\prime}$ und $P^{\prime \prime}$ bzw. $l^{\prime} \perp e_{1}$ und $l^{\prime \prime} \perp r_{2}$. - Ist $\mathrm{E}$ durch drei Punkte $A, B, C$ gegeben, so lege man etwa durch $B$ die Hauptlinien $m$ und $n$ der $E\left(m^{\prime \prime}\right.$ und $\left.n^{\prime} \| r\right)$, daun ist nach dem zweiten Satze in Art. $30 l^{\prime} \perp m^{\prime}$ und $l^{\prime \prime} \perp n^{\prime \prime}$ (Figr. 33).

Aufgabe. Die Entfernung des Punktes $P$ von der Ebene E zu bestimmen. Man ermittelt den Fuppunkt $Q$ des Lotes von $P$ auf E (Art. 28) und die wahre Länge von $P^{\prime} Q$ (Art. 19).

32. Grundaufgabe. Durch den Punkt $P$ eine Ebene zu legen senkrechtzur Geraden g (Fig. 34). Bezeichnet man mit $m$ die erste, mit $n$ die zweite durch ' $P$ 'gehende Hauptlinie der gesuchten Ebene $E$, so sind $m^{\prime \prime}$ und $n^{\prime} \| x$, und nach dem zweiten Satze in Art. 30 ist $m^{\prime} \perp g^{\prime}$ und $n^{\prime \prime} \perp g^{\prime \prime}$. Durch $m$ und $n$ ist aber $\mathrm{E}$ bestimmt. - Hierauf 
beruht die Lösung der Aufgabe: Die Entfernung des P'unktes $P$ von der Geraden!" zu bestimmen, denn die Ebene E schneidet? im Fußpunkt des von $l^{\prime}$ auf $g$ gefällten Lotes.

Anmerkung. Um die schiefe Projektion des Lotes zu zeichnen, das von einem Punkte auf eine Gerade oder auch auf eine Ebene gefällt ist. sind wir vorläufig noch gezwungen, die Aufgabe für dieselben I laten zuerst in senkrechter Projektion zu lösen und vom Fubpunkte des so gefundenen Lotes nachträglich die schiefe Projektion zu ermitteln (vgl. jedoch Art. 43 u. 4 f).

33. Aufgabe. Die kürzeste Entfernung zweier windschiefen Geraden $g$ und $h$ zu bestimmen, $d$. h. diejenige zwischen $g$ und $h$ liegende Strecke, die auf beiden Geraden senkrecht steht (Fig. 35). Man lege durch $g$ eine Ebene $\mathrm{E}\left(e_{1} e_{2}\right) \| h: \mathrm{zu}$ dem Zwecke ziehe man durch irgendeinen Punkt von $g\left(\%\right.$ B. $\left(\boldsymbol{r}_{2}\right)$ eine Parallele $i$ zu $h$. Dann fälle man von einem beliebigen Punkte $A$ von $h$ auf $\mathrm{E}$ das Lot $l$ und bestimme seinen Schnittpunkt $B$ mit E. Zieht man jetzt $B C \| h$ bis $g$ und $C D \| l$ bis $h$, so ist $C D$ die gesuchte kürzeste Entfernung, deren wahre Länge noch konstruiert werden mub.

Einfache Sonderfälle dieser Fig. 35.

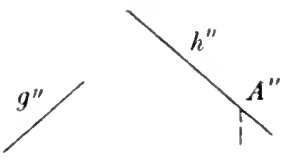
A ufgabe: a) Ist $g \perp \Pi_{1}, h$ beliebig, so ist $C^{\prime} D^{\prime}$ das Lat von $g^{\prime}$ auf $h^{\prime}$ und zugleich die wahre Länge von $C D$. b) Sind $g$ und $h \| \Pi_{1}$, so ist $C^{\prime} D^{\prime}=g^{\prime} \times h^{\prime}$ und $C D=C^{\prime \prime} D^{\prime \prime}$.

Anmerkung. Die Wahrnehmung, daß die Lösung der letzten Aufgabe sich auffallend einfach gestaltet, wenu die von den gegebenen Geraden gebildete Figur eine besondere Lage zu einer der Projektionsebenen hat, legt den Gedanken nalıe, die Aufgabe im allgemeinen Falle durch Einführung neuer Projektionsebenen zu lösen. Diese Transformation der Projektionsebenen ist überhaupt ein wichtiges Hilfsmittel zur Vereinfachung verwickelter Aufgaben.

Das in Art. 11 und 12 dargelegte Verfahren für die Ermittelung neuer Projektionen einer Figur ans zwei vorhandenen geht von der Annahme aus, daß jede neue Projektionsebene auf der vorhergehenden senkrecht steht. Man kann daher durch Einführung einer neuen Projektionsebene $\Pi_{3}$ stets erreichen, daß eine gegebene Gerade $g$ zu ihr parallel ist, indem man nämlich die $\Pi_{3}$ zu einer projizierenden Ebene von $g$ parallel stellt. Dagegen bedarf es zweier neuen Projektionsebenen, wenn die Gerade $g$ auf einer von ihnen senkrecht sein soll, nämlich zunächst einer $\Pi_{3}$ parallel zu einer projizierenden Ebene von $g$ und darauf einer $\Pi_{4}$, die auf $g$ (und daher auch auf $\Pi_{3}$ ) senkrecht steht. - Andererseits kann man unmittelbar eine $\Pi_{3}$ einführen, senkrecht zu einer gegebenen Ebene $E$, nämlich senkrecht zu einer ihrer Spuren, und dann, wenn nötig, noch eine $\Pi_{4}$, die zu $E$ parallel ist. 
Neigungswinkel einer Ebene gegen die Projektionsebenen.

34. Fallinien einer Ebene sind die Geraden der Ebene, die auf einer Spurlinie senkrecht stehen. Ist $f$ eine erste Fallinie von $E$, so ist $f^{\prime} \perp e_{1}$. Die ersten Fallinien baben unter allen Geraden der Ebene die gröbte Neigrung gegen die $\Pi_{1}$; sie bestimmen daber den Neigungswinkel der Ebene gegen $\Pi_{1}, \varepsilon_{1}=-t f^{\prime}$. (Erste Tafelneigung, Horizontalneigung der Ebene.)

Grundaufgabe. Die Tafelneigungen $\varepsilon_{1}, \varepsilon_{2}$ der Ebene $E\left(e_{1} e_{2}\right)$ zu bestimmen (Fig. 36). Eine Normalebene zu $e_{1}$ schneidet

Fig. 36.

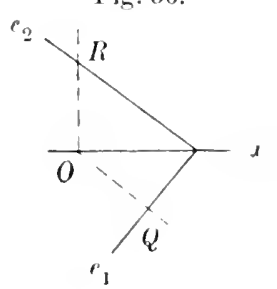

$\Pi_{1}$ in $O Q \perp e_{1}, \Pi_{2}$ in $O l \perp \perp x$ und $\mathrm{E}$ in der Falllinie $Q R \perp e_{1}$. Dimn ist $\angle O Q R=\varepsilon_{1}$, und man erhält seine wahre Giröße durch Umlegen des rechtwinkligen Dreiecks $R O Q$ entweder um $O R$ in $\Pi_{2}$, oder um $O Q$ in $\Pi_{1}$. - Ebenso ergibt sich $\varepsilon_{2}$ durch einen Normalschnitt zu $e_{2}$.

Da $\varepsilon_{1}$ und $\varepsilon_{2}$ komplementär sind zu den entsprechenden Tafelueigungen einer auf $E$ senkrecliten Geraden, so folgt aus Art. 18, dab $\varepsilon_{1}+\varepsilon_{2} \geqq 90^{\circ}$ ist.

Ist $\mathrm{E}$ durch $e_{1}$ und einen Punkt $P$ gegeben, so findet man $\angle \varepsilon_{1}$ mittels der Fallinie $P J \perp e_{1}\left(P^{\prime} J \perp e_{1}\right)$ und durch Umlegung des rechtwinkligen "Neigungsdreiecks" $P P^{\prime} J$ um $P^{\prime} J$ in $\Pi_{1}$, oder

Fig. 37 .

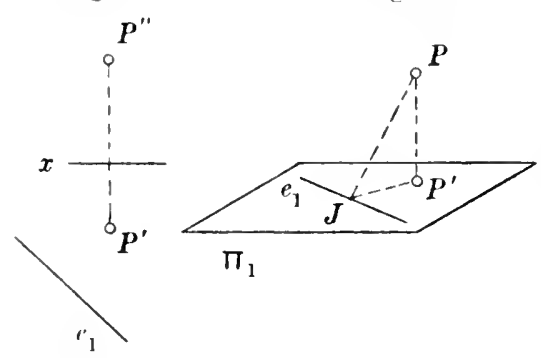
durch Drehung um $Y^{\prime} I^{\prime}$, bis es 1| $\Pi_{2}$ wird. Von diesem Dreieck kennt man die Katheten $P^{\prime} J$ und $P P^{\prime}=P^{\prime \prime} P_{x} . \quad$ Fig. 37 u. $37 \mathrm{a}$. Bei der in schiefer Projektion gezeichneten Fig. 37a ergibt sich $P^{\prime} J$ durch Umlegung der $\Pi_{1}$ in die Zeichenebene $\Pi_{2}$.)

Das Verhältnis $P P^{\prime}: P^{\prime} J$ $=\operatorname{tg} \varepsilon_{1}$ heißt die Böschung der Ebene; $\varepsilon_{1}$ wird darum auch als ihr Böschungswinkel bezeichnet.

Häufig bestimmt man eine Ebene durch ihre Spur $e_{1}$ und den Böschungswinkel $\varepsilon_{1}$; dann muß man aber linzufügen, in welchem Sinne dieser Winkel mit $\Pi_{1}$ gebildet wird.

35. Schneidet man die Ebene E (mit der Grundrißspur $e_{1}$ und der Horizontalneigung $\varepsilon_{1}$ ) in der Höhe $h$ über $\Pi_{1}$ durch

Fig. 38.

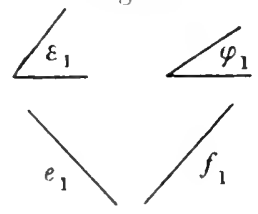
eine horizontale Ebene in der Hauptlinie $m \| e_{1}$, so ist der Abstand $m^{\prime} e_{1}$ die zwoite Kathete eines rechtwinkligen Neigungsdreiecks, in dem die Kathete $h$ dem Winkel $\varepsilon_{1}$ gegenüberliegt.

Dies dient zur Lösung der Grundaufgabe: Die Schnittlinie $g$ der Ebenen $\mathrm{E}\left(e_{1}, \varepsilon_{1}\right)$ und $\Phi\left(f_{1}, \varphi_{1}\right)$ zu bestimmen (Fig. 38). Schneidet man beide Ebenen in beliebiger Höhe $h$ über $\Pi_{1}$ mit der horizontalen Hilfsebene $\Sigma$ in den 
Hauptlinien $m$ und $n$, so geht $g$ durch den Punkt $m \times n$ und überdies durch $e_{1} \times f_{1}$. - Bei der Ausführung der Konstruktion ist der Aufriß entbehrlich.

Ist $-\varepsilon_{1}=-\varphi_{1}$, so halbiert $g^{\prime}$ den Winkel $e_{1} f_{1}$.

36. Anwendungen. a) Den Durchschnitt zweier Gräben von der 'Tiefe $t$, den in $\Pi_{1}$ liegenden Sohlen $a \| b$ und $c \| d$ und den Böschungswinkeln $\varphi$ und $\psi$ zu konstruieren (Fig. 39). Man

Fig. 39.
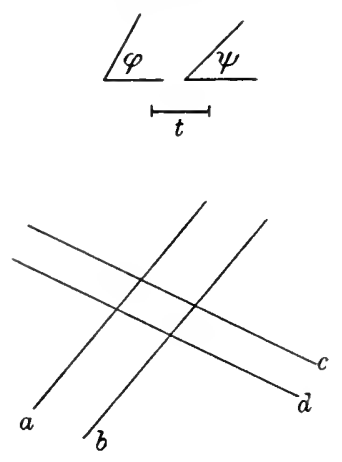

Fig. 40.

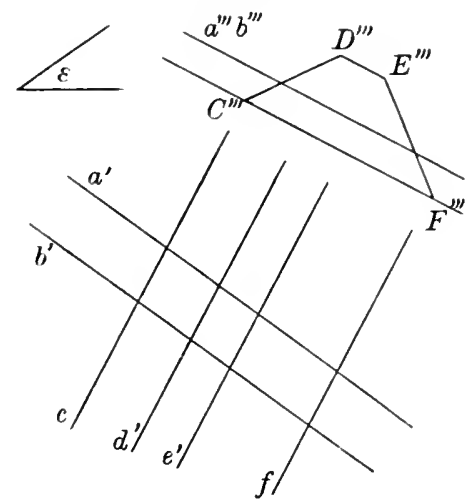

zeichne (nur im Grundriß) die Schnittlinien $e, f \| a, b$ und $g, h \| c, d$ der Böschungsflächen der Gräben mit der Ebene des Erdbodens usw.

b) Durchschnitt eines Eisenbahndammes mit einem Festungswall (Fig. 40). Der Wall ist durch seinen Normalschnitt C $D E F$ gegeben; vom Damm kennt man die Krone $a b$ und den Böschungswinkel $\varepsilon$. Lnter demselben Winkel soll der entstehende Einschnitt abgeböscht werden. - Der Seitenriß liefert dieSchnittpunkte der Geraden $a, b$ mit den Seitenflächen des Walles. Man zeichne ferner die Grundribspuren der Dammböschungen und die Schnittlinien der Einschnittsböschungen mit der horizontalen Ebene $d e$. Daraus ergeben sich die Schnittlinien der vier Böschungsflächen mit den Seitenflächen des Walles.

c) Über einem durch die Ebene $\mathrm{E}\left(e_{1}, \varepsilon_{1}\right)$ dargestellten abfallenden Gelände soll in der Höhe $h$ über der Bodenebene $\Pi_{1}$ ein horizontaler Platz

Fig. 41.

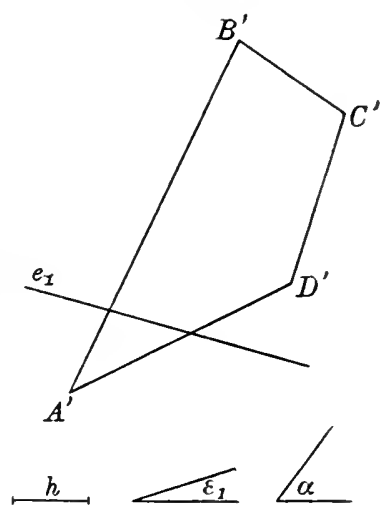
$A B C D$ unter dem Winkel $\alpha$ abgeböscht werden (Fig.41). Man ermittle zunächst die Schnittlinie $m$ der horizontalen Ebene $A B C D$ mit $\mathrm{E}$ und ihre Schnittpunkte $F$ und $G$ mit $A B$ und $C D$. Durch die Geraden $F A, A D, D G$ sind die Böschungsflächen I, II, III der aufzuschüttenden Erdmasse, durch $F B, B C, C G$ die Böschungsflächen IV, V, VI des abzutragenden Geländes zu legen. 
Von diesen sechs Ebenen bestimme man nach dem Satze in Art. 35 die Grundrißspuren s, $t, u ; v, w, z$. Dann findet man sofort die Schnittlinien $I \times I I, I I \times I I, I V \times V$, $V \times V I$, sowie die Schnittlinien von $E$ mit I, III, IV nnd VI. Die Schnittlinie von II und $E$ verbindet die Punkte $t \times e_{1}$ und $A D \times m$, und die drei Schnittlinien von II, III und $E$ treffen sich in einem Punkte. Ebenso geht die Schnittlinie von $\mathrm{V}$ und E durclı die Punkte $w \times e_{1}$ und $B$ (' $\vee m$.

37. Grundaufgabe. Durch die Gerade $g$ eine Ebene zu legen, die mit der $\Pi_{1}$ den Winkel $u$ bildet (Fig.42). Sei $P$ ein

Fig. 42.
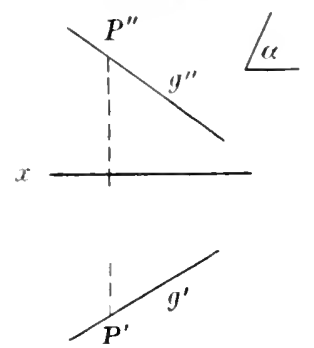
beliebiger Punkt von $\%$ Alle Ebenen, die durch $l^{\prime}$ gehen und mit der $\Pi_{1}$ den Winkel $\alpha$ bilden, sind die Berïhrungsebenen eines geraden Kreiskegels mit der Achse $P P^{\prime}$ und dem Basiswinkel $\alpha$; ihre Grundrißspuren berïhren also den Grundkreis $k$ dieses "Böschungskegels". Der Radius $r$ von $k$ ist die zweite Kathete eines rechtwinkligen Dreiecks, in dem die Kathete $P P^{\prime}=P^{\prime \prime} P_{x}$ dem Winkel $\alpha$ gectenüberliegt. - Die Grundrißspur der gesuchten Ebene geht durch den ersten Spnrpunkt von $g$ und berührt $k$.

Anwendung. Anfstieg auf einen Damm (Fig. 43). Der Damm sei gegeben durch seine Projektion $a^{\prime} b^{\prime} c d$ auf die Bodenebene $\Pi_{\mathbf{I}}$ und den Böschungswinkel $\alpha$ (Abstand $a^{\prime} c=$ Abstand $b^{\prime}(l)$. Senkrecht zur

Fig. 43.

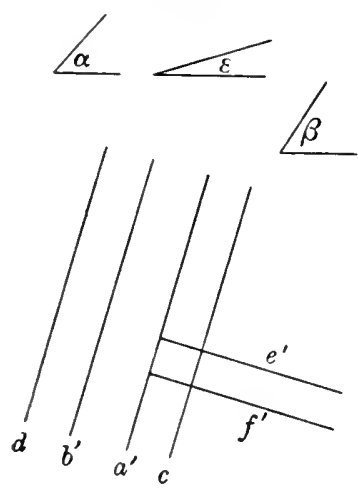

Kronenkante a soll unter dem gegebenen Neigungswinkel $\varepsilon$ der Weg ef' aufgeschïttet und unter dem Winkel $\beta$ abgeböscht werden. Man ermittle zunächst die Höhe $h$ des Dammes, darauf die Spurpunkte $E$ und $F$ der Wegkanten $e$ und $f$. Die Grundrißspuren der durch $e$ und $f$ gehenden Böschungsflächeu ergeben sich nach der vorigen Aufgabe mit Hilfe von Böschungskegeln.

\section{Drehung einer Ebene um eine Spur oder um eine Hauptlinie.}

38. Fine ebene Figur erscheint in senkrechter Projektion nur dann in wahrer Größe, wenn sie zur betreffenden Projektionsebene parallel liegt ${ }^{1}$ ). Ist dalıer eine ebene Figur in beliebiger (schräger) Lage in Grund- und Aufriß dargestellt, so findet man ihre wahre Gröbe, indem man sie zu einer Projektionsebene parallel macht oder ganz in diese mmlegt. Die erste Lagenveränderung wird bewirkt durch Drehung um eine zur betreffenden Projektionsebene parallele Hauptlinie, die zweite durch Drehung um die entsprechende Spur.

1) In schiefer Projektion erscheint die Figur anch dann in wahrer Gröbe, wenn die projizierenden Strahlen auf eiuer der beiden Ebenen senkrecht stehen, welche die Winkel \%wischen Original- und Bildebene halbieren. 
Bei Darstellungen in schiefer Projektion auf die $\Pi_{2}$ als Zeichenebene dreht man naturgemäß um eine zweite Hauptlinie oder um die $z$ we ite Spur.

39. Grundaufgabe. Die durch ihre s'pur $e_{1}$ und den Punkt $l^{\prime}$ bestiminte Ebene $E$ um $e_{1}$ in $\Pi_{1}$ umzulegen (vgl. Fig. 37 und 37 a). Der Punkt $P$ beschreibt einen Kreisbogen in einer auf $e_{1}$ senkrechten Ebene $11 \mathrm{~m}$ den Fulpunkt $J$ des Lotes von $l^{\prime}$ auf $e_{1}$. Dann ist auch $P^{\prime} J \perp e_{1} ; P$ bewegt sich also in der Ebene $P^{\prime} P^{\prime} . J$ und gelangt nach $P_{0}$ auf der Geraden $P^{\prime} J$, so daß $P_{0} J=P J$ wird, d. h. gleich der Hypotenuse des rechtwinkligen Dreiecks $P P^{\prime} J$ mit den bekannten Katheten $P^{\prime} J$ und $P^{\prime} P^{\prime}=P^{\prime \prime} P_{x}$.

Für die Umlegung einer ebenen Figrur in eine Ebene gelten hiernach die Sätze: 1. Die Umlegung jedes Punktes und seine senkrechte Projektion auf die Ebene liegeu immer in einem Lote zur Drehungsachse.

2. Der Abstand dieser Umlegung von der Drehungsachse ist die Hypotenuse eines rechtwinkligen Dreiecks, welches das vom Punkte auf die Ebene gefällte Lot und den Abstaud seines Fußpunktes von der. Drehnngsachse zu Katheten hat.

Dazu kommt noch: 3. Die Umlegung jeder Geraden geht durch den Schnittpunkt der Geraden mit der Drehungsachse.

Anwendung. Die wahreGröbedes Winkels

Fig. 44.
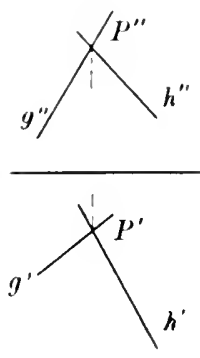
zweier im Punkte $P$ sich schneidenden Geraden $g$ und $h$ und die Projektionen seiner Halbierungslinie zu bestimmen (Fig. 44). Man konstruiere von $g$ und $h$ die Spurpunkte $G_{1}$ und $H_{1}$ und vom Dreieck $P G_{1} H_{1}$ seine Umlegung in $\Pi_{1}$. Darauf halbiere man den Winkel $G_{1} P_{0} H_{1}$ usw.

Fig. 45 .

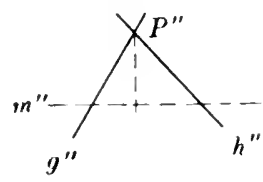

Fig. 46.
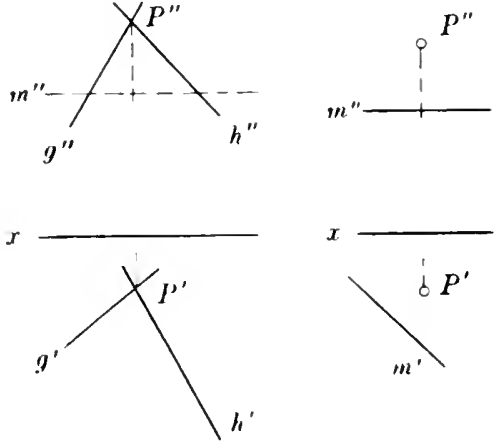

Fig. 46 a.

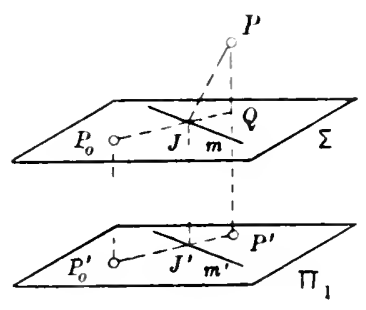

m

40. Ist der Punkt $P$ von der Spurlinie $G_{1} H_{1}$ der Ebene gh zu weit entfernt, so dreht man die Ebene besser um eine erste Hauptlinie $m$, bis sie $\| \Pi_{1}$ wird (Fig. 45). Dabei ist $m^{\prime \prime} \| x$ passend $z u$ wählen; daraus ergibt sich sofort $m^{\prime}$. 
Ihies fülırt zu der weiteren Grundaufuabe: Die durch die horizontale Gerade $m$ und den Punlit $P$ bestimmte Ebene E durch lorehung um $m$ in horizontale Lage zu bringen (Fig. 46 und Skizze $t 6$ a in schiefer Parallelprojektion). Ist $\Sigma$ die durch $m$ gehende Horizontalebene, (? ihr Schnittpunkt mit $I^{\prime} P^{\prime}, J$ der Fußpunkt des Lotes ron $P$ auf $m$, so ist auch $(Y, J \perp m$, und man erbält die Um$\operatorname{leguug} P_{0}$ von $P$ in $\Sigma$, indem man $J P$ von $J$ aus auf $Q, J$ abträgt. Da $Q^{\prime}$ mit $P^{\prime}$ zusammenfallt, so ist das Lot von $I^{\prime \prime}$ anf $m^{\prime}$ der Grundriß von $Q . J$. Nan ziehe also $I^{\prime} J^{\prime} \perp m^{\prime}$ und mache $J^{\prime} I_{0}^{\prime}$ gleich der Hypotenuse eines rechtwinkligen Dreiecks mit den Katheten $P^{\prime} J^{\prime}$ und $P^{\prime \prime} Q^{\prime \prime}$.

4l. Beziehungen zwischen der Gröbe ejnes Winkels und derGrößeseiner senkrecliten Projektion. Sei $B A C$ ein spitzer

Fig. 47.

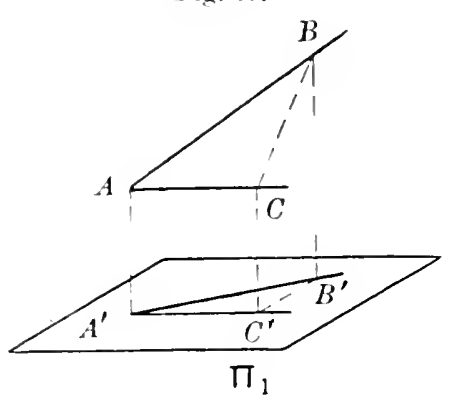

Winkel und $A C \| \Pi_{1}$ (Fig. 47). Zieht man $l ; C \perp A C$, so ist auch $l^{\prime} C^{\prime} \perp A^{\prime} C^{\prime}$ (Art. 30). In den rechtwinkligen Dreiecken $b^{\prime} A^{\prime} C^{\prime}$ und $B A C$ sind die Katheten $A^{\prime} C^{\prime}$ und $A C$ einander gleich, dagegen ist $B^{\prime} C^{\prime}<B C$, folglich auch $\angle A^{\prime}<\angle A$. D. h.: Ein spitzer Winkel, dessen einer Schenkel zur Projektionsebene parallel ist, wird durch senkrechte Projektion verkleinert.

Aus denselben Dreiecken ergibt sich ferner, daf $<B^{\prime}>-B$ ist. In Worten: Ein spitzer Winkel, dessen einer Schenkel eine Falllinie seiner Ebene ist, wird durch senkrechte Projektion vergröbert.

Wenn ein Winkel die durch seinen Scheitel gehende Hauptlinie einschließt, die durch denselben Punkt gehende Fallinie aber ausschließt, so wird er durch senkrechte Projektion verkleinert. Schliebt er umgekehrt die Fallinie ein, die Hauptlinie aber aus, so wird er vergrößert. Schliebt er beide Linien ein oder beide aus, so kann er verkleinert oder vergrößert werden, oder ungeändert bleiben.

42. Mit der Aufgabe, den Winkel zweier Geraden zu bestimmen, sind zugleich die folgenden Aufgaben gelöst:

a) Den Neigungswinkel $\alpha$ zu ermitteln, den die Gerade $g$ mit der Ebene E bildet. Fält man von einem beliebigen Punkte von ! auf $E$ das Lot l, so ist $\alpha=90^{\circ}--g l$.

b) Den Neigungswinkel der Ebonen $\mathbf{E}\left(e_{1} e_{2}\right)$ und $\boldsymbol{\Phi}\left(f_{1} f_{2}\right)$ zu konstruieren. Fällt man vou einem beliebigen Punkte auf $E$ und $\Phi$ die Lote $g$ und $h$, so billen diese den gesuchten Winkel.

Figr. 48 enthält eine zweite Lösung dieser Aufgrabe, dargestellt in schiefer Parallelprojektion auf die Zeichenebene $\Pi_{2}$ : Man lege senkrecht zur Schnittlinie $G_{1} G_{2}$ von $E$ und $\Phi$ eine Ebene $N$; diese schneidet $E$ und $\Phi$ in den Schenkeln des gesuchten Winkels $\alpha$. Ihre zweite Spurlinie $n_{2}$ ist $\perp G_{1}^{\prime \prime} G_{2}$ und kann im übrigen beliebig angenommen werden. Bezeichnet man mit $B, C$ und $I$ bzw. die Schnittpunkte ron 
${ }_{2}$ mit $e_{2}, f_{2}$ und $G_{1}^{\prime \prime} G_{2}$, mit $A$ den noch unbekannten Schnitpunkt von $\mathrm{N}$ und $G_{1} G_{2}$, so ist $\angle B A C=\%$. Die Gerade $D A$ ist die Schnittlinie der Ebenen $N$ und $G_{1} G_{1}^{\prime \prime} G_{2}$; sie ist die Höhenlinie im Dreieck $B A C$, weil $n_{2}$ auf der Ebene $G_{1} G_{1}^{\prime \prime} G_{2}^{\prime}$ senkrecht steht, und zugleich das Lot von $I$ auf $G_{1} G_{2}$, weil $N \perp \dot{r}_{1} G_{2}$ ist. Man erhailt daher die wahre Länge von $D A$, indem man das rechtwinklige Dreieck $G_{1} G_{1}^{\prime \prime} G_{2}$ um seine Kathete $G_{1}^{\prime} G_{2}$ in $\Pi_{2}$ unlegt: Zieht man $G_{1}^{\prime \prime} G_{1}^{0} \perp G_{1}^{\prime \prime} G_{2}$ nud $=$ der wahren Länge von $G_{1} G_{1}^{\prime \prime}$, sowie $D A^{0} \perp G_{1}^{0} G_{2}$, so ist

Fig. 48.

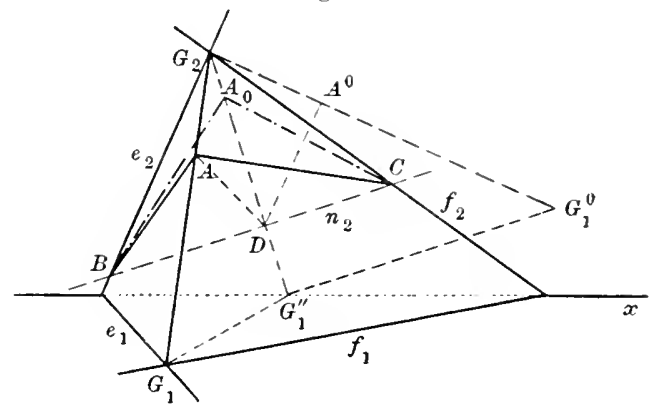

$D A=D A^{0}$. Jetzt ergibt sich die wahre Größe des Winkels $\alpha$ durch Umlegung des Dreiecks $B A C$ um $B C$ in $\Pi_{2}$. Dabei fällt die Höhenlinie $D A$ in die Gerade $G_{1}^{\prime \prime} G_{2}$; macht man auf dieser $D A_{0}=I A^{0}$, so findet man $\alpha=-B A_{0} C$. (Die schiefe Projektion des Punktes $A$ liegt auf der Parallelen durch $A^{0}$ zu $G_{1} G_{1}^{0}$.)

43. Fig. 49 gibt in schiefer Parallelprojektion eine neue Lösung der bereits in Art. 31 behandelten Aufgabe, die Entfernung des Punktes $P$ von der Ebene E $\left(e_{1} e_{2}\right)$ zu bestimmen; dabei ist $P$ durch sein Bild und seinen Aufrib $P^{\prime \prime}$ gegeben. Von dem Lot $P Q$, das man von $P$ auf $E$ zu fällen hat, kann man bei der gewählten Darstellungsweise zunächst nur den Aufrib zeichnen: dieser ist $\perp e_{2}$ und trifft $e_{2}$ in $R$ und $x$ in $S$. Die zugehörige zweite projizierende Ebene schneidet $\Pi_{1}$ in $S T \perp x$ und $\mathrm{E}$ in $R T$, und dann ist $P Q$ das Lot von $P$ auf die Gerade $L^{\prime} T$. Um das Lot in Fig. 49.

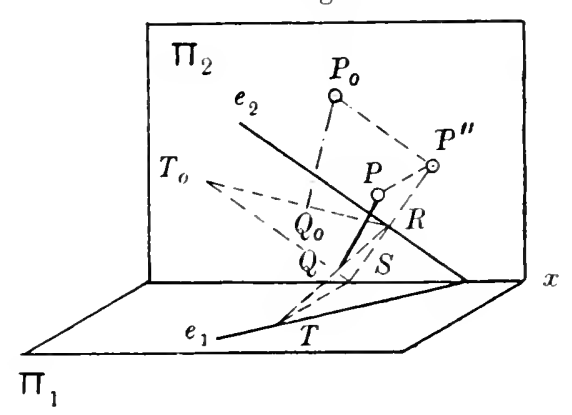
wahrer Größe und darauf auch in schiefer Projektion zu zeichnen, lege man seine zweite projizierende Ebene in die $\Pi_{2}$ um. Gelangen dadurch die Punkte $P$ und $T$ nach $P_{0}$ und $T_{0}$, so ist $P^{\prime \prime} P_{0} \perp P^{\prime \prime} S$ und $=$ der wahren Länge von $P^{\prime \prime} P$. Dann ist $P_{0} Q_{0} \perp R T_{0}$ die wahre Größe der gesuchteu Entfernung. Die schiefe Projektion des Fußpunktes $Q$ ergibt sich durch $Q_{0} Q \| T_{0} T$. 
Bei Anwendung senkrechter Projektion kann man natïrlich an Stelle der zweiten auch die erste projizierende Ebene des Lotes benutzen.

44. Auch die in Art. 32 erwänte Aufgabe, die Entfernungr des Punktes $l^{\prime}$ yon der Geraden $g$ zu bestimmen, kann jetzt in anderer Weise gelöst werden. In Fig. 50 sind $I^{\prime}$ und g nebst ihrem Grundrib $I^{\prime}, g^{\prime}$ in schiefer Projektion gegeben. Die gesnchte Entfernung wird durch das Lot $P Q$ vou $I^{\prime}$ auf g gemessen. Um nun den rechten Winkel zwischen $P Q$ und $g$ in schiefer Projektion in wabrer Gröbe

Fig. 50.

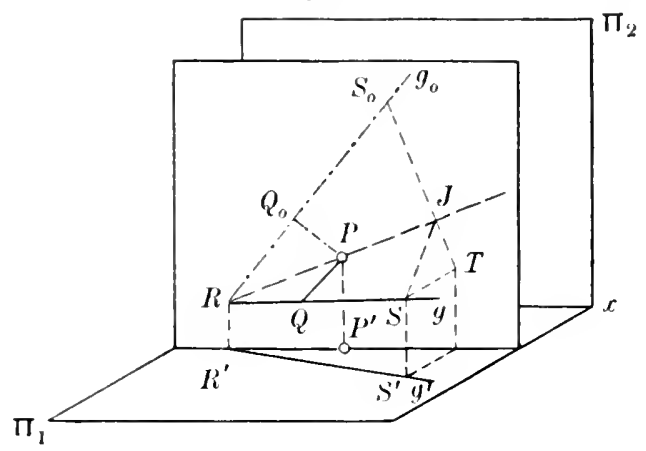

zeichnen zu können, muß man die Ebene $P g$ vorher drehen, bis sie $\| \Pi_{2}$ wird. Diese Drehung erfolgt um eine zweite Hauptlinie, die man zweckmäßig durch den Punkt $P$ legt; zieht man $H^{\prime} K^{\prime} \| x$ lis $g^{\prime}$, so ist $P R$ das Bild der Drehungsachse. Bei der Drehung bleiben die Punkte $P$ und $R$ fest, es ist also nur noch die neue Lage $S_{0}$ eines beliebigen Punktes $S$ der Geraden $g$ zu bestimmen. Bezeichnet man mit $T$ ' den Schnittpunkt des zweiten projizierenden Strahls von $S$ mit der Ebene $P R R^{\prime} P^{\prime}$, mit $J$ den Fubpunkt des Lotes von $T$ auf $I^{\prime} R$, so erhält man $S_{0}$, indem man die Strecke $J S$, d. h. die Hypoteuuse des recbtwinkligen Dreiecks $S T J$, dessen Katheten bekannt sind, von $J$ aus auf $T J$ abträgt. I)ann ist die Gerade $R S_{0}$ die Umlegung von $g$, mithin liefert $P Q_{0} \perp R S_{0}$ den gesuchten Abstand in wahrer Größe.

45. Aufgabe. Die wabre Gröbe des durch Grund-und Aufriß gegebenen Dreiecks $A B C$ zu bestimmen (Fig. 51). Man

Fig. 51 .

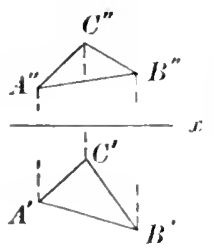
drehe die Ebene $A B C$ nicht um eine Spur, sondern um eine Hauptlinie, die durch einen Eckpunkt des Dreiecks gelegt wird, \%. B. um die zweite Hauptlinie $n$ durch $A$, bis sie $\| \Pi_{2}$ wird. Die Umlegungen der Eckpminte $B$ und $C$ werden dann genan so gefunden, wio die des Punktes $S$ in der vorhergehenden Aufgahe.

46. Grundaufgabe. Ein ebenes Vieleck $A B C \ldots$ ist durch die Spuren $e_{1}, e_{2}$ seiuer Ebono E und seine erste Projektion $A^{\prime} B^{\prime} C^{\prime} \ldots$ gegeben; sejne zweite Projektion und seine wahre Gröbe zu bestimmen (Fig. 52). Erste Lösung: Man konstruiere zuerst die zweiten Projektionen der Eckpunkte mit Hilfe von Hauptlinien (Art. 25), 
oder sogleich die zweiten Projektionen einzelner Seiten (Art. 23). Darauf ergibt sich die wahre Gröbe des Vielecks durch Unlegung der Ebene $\mathrm{E}$, etwa um $e_{1}$ in $\Pi_{1}$. Hat man von einem Eckpunkt $A$ (am zweckmäBigsten von demjenigen, der voll $e_{1}$ an weitesten entfernt ist) nach Art. 39 die Imlegung $A_{0}$ ermittelt, so geht $A_{0} B_{0}$ nach dem Sohnittpunkte von $A^{\prime} B^{\prime}$ mit $e_{1}$ usw.

Eine zweite Lösung beruht auf der Einführungr einel. neuen Projektionsebene $\Pi_{3}$, die auf $E$ und a uf $\Pi_{1}$ oder $\Pi_{2}$ senkrecht steht.

Nehmen wir die $\Pi_{3}$ z. P. $\perp \Pi_{1}$, also auch $\perp e_{1}$, und bestimmen sie durch ihre Schnittlinie init $\Pi_{1}, y=O Q \perp e_{1}$, so schneidet sie die $\Pi_{2}$ in $O R \perp x$ und die $E$ in der Fallinie $e_{3}=Q R \perp e_{1}$ (vgl. die Skizze 53

Fig. 52.

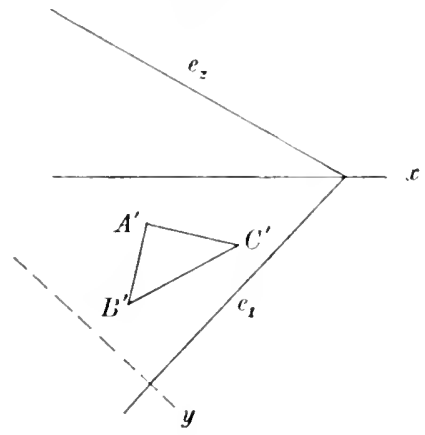

Fig. 53.

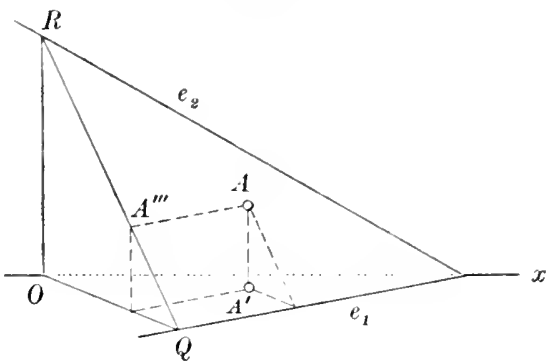

in schiefer Parallelprojektion). Durch Umlegung der $\Pi_{3}$ in die $\Pi_{1}$ gelangt das rechtwinklige Dreieck $O Q R$ nach $O Q R^{0}$, wobei $O R^{0} \perp O Q$ und $=O R$ ist. Da $\mathrm{E}$ auf $\Pi_{3}$ senkrecht steht, so liegen die dritten Projektionen aller Punkte der $\mathrm{E}$ a uf $e_{3}$, und zwar ist $A^{\prime} A^{\prime \prime \prime} \perp y$. Dann ergibt sich $A^{\prime \prime}$ nach Art. 11 aus der Bedingung: Abstand $A^{\prime \prime} x$ $=$ Abstand $A^{\prime \prime \prime} y$. Ferner ist nach Art. $39 A^{\prime} A_{0} \perp_{e_{1}}$ und Alostand $A_{0} e_{1}=Q A^{\prime \prime \prime}$.

Fällt $R$ oder $R^{0}$ in unerreichbare Entfernung, so findet man die Umlegung von $e_{3}$, indem man von irgendeinem Punkte der $\mathbf{E}, z$. B. von einem beliebigen Punkte $S$ der spur $e_{2}$, die dritte Projektion ermittelt: $S S^{\prime} \perp x, S^{\prime} S^{\prime \prime \prime} \perp y$, Abstand $S^{\prime \prime \prime} y=S S^{\prime}$.

Ebenso verfährt man, wenn $E$ nicht durch $c_{1}$ und $e_{2}$, sondern durch $e_{1}$ und einen beliebigen Punkt $P$ bestimmt ist.

$\angle e_{3} y$ ist gleich der ersten Tafelneigung der Ebene $\mathrm{E}$.

Kenut man ungekehrt von $\operatorname{dem}$ Vieleck $A B C \ldots$ seine Umlegung $A_{0} B_{0} C_{0} \ldots$, so erhält man seinen Grund- und Aufrib durch Zurückdrehen, indem man dieselben Konstruktionslinien in ungekehrter Reihenfolge zeichnet.

47. Grundaufgabe. Von einem ebenen Vieleck kennt man die erste Hauptlinie $m$ und den ersten Neigungswinkel $\varepsilon_{1}$ seiner Ebene $E$, sowie die erste Projektion $A_{0}^{\prime} B_{0} C_{0}^{\prime \prime} \ldots$ seiner Umlegung in die durch $m$ gehende Horizontalebene $\Sigma$; die Projektionen des Vielecks zu bestimmen (Fig. 54). Eine dritte 
Projektionsebene $\Pi_{3} \perp m$ schneidet die $\Sigma$ in einer Geraden $y \perp m\left(y^{\prime} \perp m^{\prime}\right)$ und die $E$ in einer Fallinie $e_{3}$, so dab $-e_{3} !=\varepsilon_{1}$ ist. Wird die $\Pi_{3}$ nm y in $\Sigma$ umgelegt, so erhält man den (irundrib $e_{3}^{\prime}$ der gedrehten Fall-

Fit. 54 .

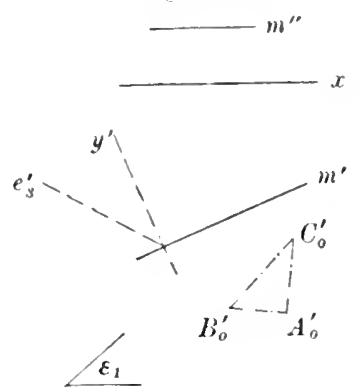

linie, indem man $-\varepsilon_{1}$ in $Q^{\prime}=\prime^{\prime} \times y^{\prime}$ in gegebenem Sinne an $y^{\prime}$ anträgt. Jlacht man auf $\iota_{3}^{\prime}$ die Strecke $\left(Q^{\prime} A^{\prime \prime \prime}=\right.$ Abstand $A_{n}^{\prime} m^{\prime}$, so ergibt sich $A^{\prime}$ als Schnittpunkt der Lote von $A_{0}^{\prime}$ auf 'l' ${ }^{\prime}$ und ron $A^{\prime \prime \prime}$ anf $y^{\prime}$, und es ist Abstand $A^{\prime \prime} m^{\prime \prime}=$ Abstand $A^{\prime \prime \prime} y^{\prime}$.

48. Anwendungen. a) Zwei Ebenen $E$ und $\phi$ sind durch ihre Grundribspuren $e_{1}$ und $f_{1}$ und durch je einen Punkt bzw. $A$ und $B$ gegeben. Es soll der kürzeste Weg von $A$ nach $B$ gefunden werden, der in $E$ vou $A$ bis $e^{\prime}$ : dann in $\Pi_{1}$ bis $f_{1}$ und schließlicb in $\Phi$ bis $B$ verläuft (Fig. 55). Klappt man $E$ und $\Phi$ in $\Pi_{2}$ um, so verwandelt sich jener kürzeste $W$ eg in die Gerade $A_{0} B_{0}$. Ihre Schnittpunkte $E$ und $F$ mit $c_{2}$ und $f_{1}$ liefern die gesuchte gebrochene Linie $A E F B$.

b) Dachausmittelung (Fig. 56 ). In der Anfangsebene $\Pi_{1}$ des Daches ist das Sechseck $A B C D E F$ gegeben, in dem $B C\|A F\| x$ und $C D \| F E$ ist. Durch die sechs Seiten (Trauflinieu) sollen Ebenen Fig. 55.

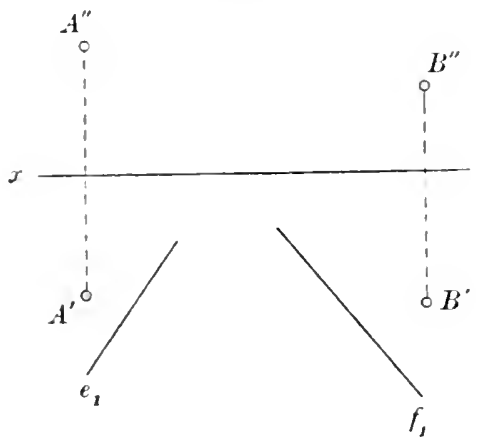

Fig. 56.

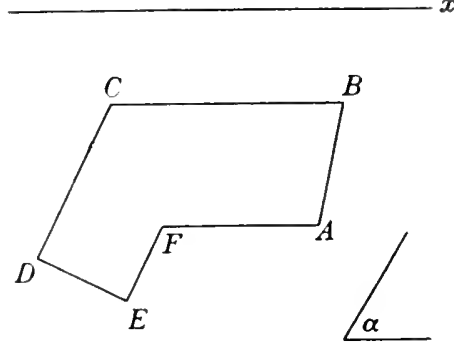

grelegt werden, die sümtlich mit $\Pi_{1}$ den Winkel $\alpha$ bilden. Von dem so entstehenden Walmdach ist zunäclast der Grundrif zu zeichnen.

Die durch $B C$ und $A F$ gehenden Dachflächen schneiden sich in der Firstlinie $G H$, deren Grundrißs ron $B C$ und $A F$ gleichweit entfernt ist. Je zwei benachbarte Dachflächen schneiden sich in einem Grat (z. B. $A G$ ) oder in einer Kehle $(F . J)$, deren erste Projektionen die Winkel zwischen den Trauflinien halbieren (vgl. Art. 35, Schluß). Der Grundriß der Verfallung $H . J$ halbiert den Winkel zwischen $A F$ und $C D$.

Man ermittele ferner die wahren Gröben der Dachtlächen durch Umlegung in $\Pi_{1}$, sowie den Aufrib des Daches: Die Abstände des 
Punktes $G$ von $A B$ und von $\Pi_{1}$ ergelsen sich als Hypotenuse und zweite Kathete in einem Neigungsdreieck mit dem Lot von $G^{\prime}$ auf $A B$ als Kathete und $\alpha$ als anliegendem Winkel.

\section{Affinität zwischen einer ebenen Figur und ihrer Parallelprojektion.}

49. Zwischen zwei ebenen Figuren, deren pine die senkrechte oder schiefe Parallelprojektion der anderen ist, besteht eine geometrische Abhängigkeit (Verwandtschaft), die als Affinität bezeirlnnet wird: Jedem Punkte und jeder durch ihn gebenden Geraden der einen Figur entspricht in der anderen bzw. ein Punkt und eine durch diesen gehende Gerade; parallelen Geraden entsprechen wieder parallele Geraden usf.

Projiziert man die in der Ebene $\mathrm{E}$ liegende Figur $A B C \ldots$ in heliebiger Richtung auf die Ebene $\Pi$, und ist $A^{\prime}$ die Projektion von $A$.

Fig. 57 .

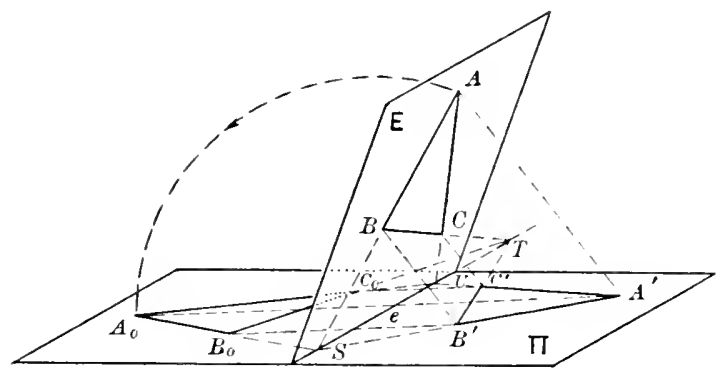

so geht das Bild rler Geraden $A B$ von $A^{\prime}$ nach ihrem Schnittpunkte $S$ mit der Spur $e$ von $E$ (Fig. 57 ). Die besondere Lage, in der sich hier die beiden affinen Figuren $A B C$.. und $A^{\prime} B^{\prime} C^{\prime} \ldots$ befinden, heibt perspektive Lage. Sie ist durch zwei Eigenschaften gekennzeichnet: 1. Alle Verbindungsinien entsprechender Punkte (Affinitätsstrahlen) sind parallel; 2. alle Schnittpunkte entsprechender Geraden liegen in einer Geraden, nämlich in der Spur e (Affinitätsachse).

In Fig. 57 verhält sich $S A: S B=S A^{\prime}: S B^{\prime}$. Bringt man die Ebene $E$ durch Drehung um $e$ in irgendeine neue Lage, so gilt dieselbe Proportion; es ist also wieter $A A^{\prime} \| B B^{\prime}$, d. h. die affinen Figuren $A B C \ldots$ und $A^{\prime} B C^{\prime} \ldots$ bleiben in perspektiver Lage. Wird die Ebene $E$ so weit gedreht, bis sie mit $\Pi$ zusammenfällt, so folgt: Die Parallelprojektion und die Cmlegung einer ebenen Figur in die Bildebene sind perspektiv affin mit der Spurlinie der Originalebene als Affinitätsachse.

Sind demnach von einer ebenen Figur $A B C \ldots$ die Spurlinie $e$, die Umlegung $A_{0} B_{0} C_{0} \ldots$ und das Bild $A^{\prime}$ eines Punktes $A$ bekannt, so ist die Bildfigur bestimmt.

Im Falle senkrechter Projektion ist $A_{0} A^{\prime} \perp e$ und Abstand $A^{\prime} e$ $<$ Abstand $A_{0} e$. 
50. Sei $A B C$ ein in $E$ liegendes Dreieck, das durch die Hauptlinie $A I)$ in die Dreiecke $A D B$ und $A D C$ zerlegt wird (Fig. 58).

Fig. 58.

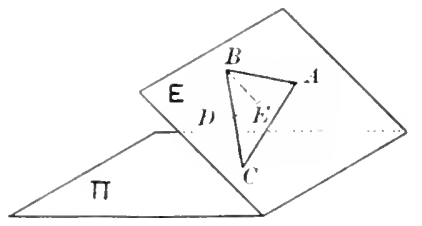
Zieht man $l, E \perp A l$, und bildet von der so erhaltenen Firur die senkrechte Projektion $A^{\prime} B^{\prime} C^{\prime} J^{\prime} E^{\prime}$ anf $\Pi$, so ist, wenn $\varepsilon$ den Neigungswinkel der Ebene $E$ gegen $\Pi$ bezeichnet, $A^{\prime} l^{\prime}=A I, B^{\prime} E \perp A^{\prime}()^{\prime}$ und $=B E \cos \varepsilon$, also $\triangle A^{\prime} D^{\prime} B^{\prime}=$ $\frac{1}{2} A^{\prime} D^{\prime} \cdot B^{\prime} E^{\prime}=\frac{1}{2} A D \cdot B E \cos \varepsilon=$ $\triangle A D B \cos \varepsilon$. Ebenso ist $\triangle A^{\prime} L^{\prime} C^{\prime}=$ $\triangle A D C \cos \varepsilon$, mithin auch $\Delta A^{\prime} B^{\prime} C^{\prime}$ $=\triangle A B C \cos \varepsilon$. $Z$ wischen len Flächeninhalten $F$ und $F^{\prime}$ einer ebenen Figur und ihrersenkrechten Projektion und der Tafelneigung $\varepsilon$ besteht demnach überhaupt die Beziehung:

$$
F^{\prime}=F \cos \varepsilon \text {. }
$$

51. Ein entsprechender Satz gilt auch im Falle schiefe r Parallelprojektion. Ist nämlicb $A B C$ ein Dreieck der Ebene E, $A^{\prime} B^{\prime} C^{\prime}$ seine Projektion auf $\Pi$ und $A_{0} B_{0} C_{0}$ seine I'mlegung in diese Ebene, so liegen die Schnittpunkte $S, T, U$ von $A_{0} B_{0}, B_{0} C_{0}, C_{0} A_{0}$ mit den entsprechenden Seiten des Dreiecks $A^{\prime} B^{\prime} C^{\prime}$ auf der Spurlinie $e$ (vgl. Fig. 57). Schneidet $e$ die Affinitätsstrahlen $A_{0} A^{\prime}, B_{0} B^{\prime}, C_{0} C^{\prime}$ bzw. in $\mathfrak{Z}, \mathfrak{B}, \mathfrak{C}$, so folgt unmittelbar aus der Figur:

$$
A_{0}^{\prime} \mathfrak{H}=\frac{B^{\prime} \mathfrak{B}}{B_{0} \mathfrak{B}}=\frac{C^{\prime} \mathfrak{S}}{C_{0} \mathfrak{S}} .
$$

Dieses für alle Punkte der Ebene E konstante Verhälnis heißt die Charakteristik $\delta$ der zwischen den Ebenen $E$ und $\Pi$ bestehenden Affinität.

Nun ist z. B.

$$
\frac{\Delta A^{\prime} S U}{\angle A_{0} S U}=\frac{A^{\prime} \mathscr{A}}{A_{0}: I^{\prime}}
$$

also $=\delta$. Bedenkt man weiter, dal.

$$
\Delta A^{\prime} B^{\prime} C^{\prime}=\Delta A^{\prime} S U+\Delta C^{\prime} T^{\prime} U-\Delta B^{\prime} S T^{\prime}
$$

ist, so ergibt sich

$$
\frac{\Delta A^{\prime} B^{\prime} C^{\prime}}{\Delta I_{0} S_{0} C_{0}}=\delta
$$

Das Verhältuis der Flächeninhalte ron Bild- und Originalfigur ist demnach überhaupt für alle in $E$ liegeuden Figuren konstant, nämlich $=d$.

52. Find $A^{\prime} B^{\prime} C^{\prime} \ldots$ und $A^{\prime \prime} B^{\prime \prime} C^{\prime \prime} \ldots$ Grund-und Aufriß eines ebenen Tielecks, so liegen im Schnittpunkte von $A^{\prime} B^{\prime}$ nnd $A^{\prime \prime} B^{\prime \prime}$ die beiden Projektionen des l'unktes vereinigt, in $\operatorname{dem} A B$ die zweite Halbierungsebene $\mathrm{H}_{2}$ schneidet (vgl. Art. 8 und Fig. 20). Das Entsprechende grilt vom Schnittpunkte von $I^{\prime} C^{\prime}$ und $I^{\prime} C^{\prime \prime}$ usf. Diese Schnittpunkte liegen aber sämtlich in einer (ieraden, nämlich in den zusammenfallenden Projektionen $w^{\prime}$ und $w^{\prime \prime}$ der Schnittlinie $*$ der 
Ebenen $A B C \ldots$ und $\mathrm{H}_{2}$. Daraus folgt: Grund-und Anfrib einer ebenen Figur sind perspektiv affin - aber selbstverständlich erst, wenn die Unlegung der vorderen $\Pi_{1}$ in die untere $\Pi_{2}$ bereits vollzogen ist.

\section{Ebenflächige Gebilde.}

Darstellung einiger. Tielflache.

53. Aufgabe. Ein gerades Prisma in Grund-und Aufriß darzustellen, wenn gegeben ist 1 . der Eckpunkt $A\left(A^{\prime}, A^{\prime \prime}\right)$; 2. vou der Girundfläche $A B C$ die durch $A$ gehende erste Hauptlinie $m$, der erste Neigungswinkel $\varepsilon_{1}$ und der Grundrif $A^{\prime} B_{0}^{\prime} C_{0}^{\prime}$ der Umlegung in die durch $m$ gehende Horizontalebene; 3 . die Länge der. Seitenkante $A D$ (Fig. 59). Man konstruiere zunächst die Projektionen der Grundfläche wie in Art. 47 mit Hilfe einer $\Pi_{3} \perp m$. Da die Kante $A D \| \Pi_{3}$ ist, so erscheint sie in dritter Projektion in wahrer Gröle und senkrecht auf der dritten Spur der Grundfläche. Dann ist $D^{\prime}$ der Schnittpunkt der Geraden $A^{\prime} D^{\prime} \perp m^{\prime}$ und $D^{\prime \prime \prime} D^{\prime} \| m^{\prime}$; ferner ist Abstand $D^{\prime \prime} m^{\prime \prime}=\mathrm{Ab}$ stand $D^{\prime \prime \prime} y^{\prime}$. Die Projektionen der Kanten $B E$ und $C F$ sind $\# A^{\prime} D^{\prime}$ bzw. $\Lambda^{\prime \prime} D^{\prime \prime}$.

Wird der dargestellte Körper als undurchsichtig vorausgesetzt, so enthält jeder projizierende Strahl, der den Körper sclnneidet, einen sichtbaren und einen unsichtbaren Punkt seiner Oberfläche. Das windschiefe Fünfeck $B C F D E$, in dem die ersten projizierenden Strahlen den Körper streifen (ohne ihn zu schneiden), heibt der erste wahre Umriß und sein Grundriß der erste schein-

Fig. 59 .

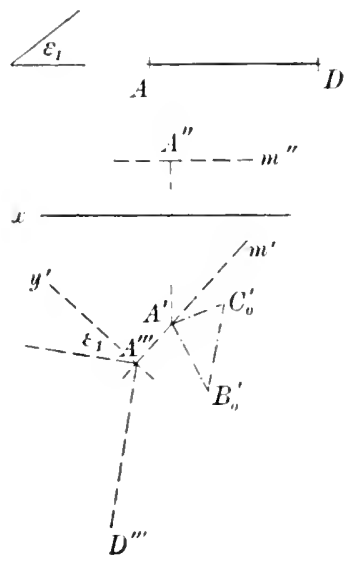

bare Umriß des Körpers. Der erste wahre Umrif trennt den im Grundrif sichtbaren Oberflächenteil vom unsichtbaren. - Die drei in $A$ zusammenstoßenden Flächen sind im Grundrib unsichtbar, denn der Aufriß zeigt, daß $A$ auf dem unteren, im Grundriß unsichtbaren Oberflächenteile liegt. Um zu entscheiden, welche der beiden Kanten $A D$ und $E F$ im Grundriß sicbtbar ist, könnte man aber auch wie in Art. 20 untersuchen, welcher Punkt dieser Kanten, dessen Grundriß mit dem Schnittpunkte von $A^{\prime} D^{\prime}$ und $E^{\prime} F^{\prime}$ zusammenfällt, höher liegt als der andere.

Für das Ausziehen der Figur ist noch zu beachten: Der scheinbare Umriß ist stets sichtbar. Die Bilder zweier sichtbaren oder zweier unsichtbaren Kanten dürfen sich zwischen ihren Endpunkten niemals schneiden. Die von einem nicht dem Unriß angehörenden Punkte ausgehenden Kanten sind entweder sämtlich sichtbar oder sämtlich unsichtbar, je nachdem der Punkt sichtbar oder unsichtbar ist.

54. Aufgabe. Ein Tetraeder zu konstruieren aus der in $\Pi_{1}$ liegenden Grundfläche $A B C$ und den Längen $a, b, c$ der drei Seitenkanten $A D, B D, C D$ (Fig. 60). Zeichnet man in $\Pi_{1}$ 
die Dreiecke $A B D_{0}$ und $B C D^{0}$ als Umlegungen der Flächen $\left.A L I\right)$ and $B\left(C D\right.$, so ergibt sich $l^{\prime}$ als Schnittpunkt (ler Lote von $D_{0}$ auf $A B$ und von $D^{0}$ auf $B C$. Schneidet $D_{0} D^{\prime}$ die Gerade $A B$ in $J$, so ist

Fig. 60.
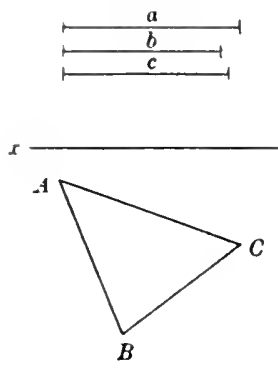
$D^{\prime \prime} D_{r}$, d. h. der erste Tafelabstand von $D$, gleich der zweiten Kathete eines rechtwinkligen Dreiecks mit der Fathete $J I^{\prime}$ und der Hypotenuse $J D_{0}$, oder auch eines rechtwinkligen Dreiecks mit der Kathete $1 D^{\prime}$ und ler Hypotenuse $a$.

55. A ufgabe. Kin regelmäbiges Zwölfflach darzustellen, dessen eine Fläche $A_{1} A_{2} A_{3} A_{4} d_{5}$ in $\Pi_{1}$ liegt (Fig. 61). Die übrigen Eckpunkte des hörper's befinden sich zu je fünf in drei horizontalen Ebenen und bilden in ihnen drei regelmälige Fünfecke, die wir in der Reihenfolge von unten nach oben mit $B_{1} \ldots B_{5}, C_{2} \ldots C_{5}$, $D_{1} \ldots D_{5}$ bezeichnen. Dabei soll $B_{1}$ denjenigen der Punkte $B$ bedeuten, der mit $A_{1}$ durch eine Kante verbunden ist. Die Punkte $A$ und $D$, sowie die Punkte $B$ und $C$ liegen paarweise auf 10 Geraden, die sich im Mittelpunkte $M$ des Körpers halbieren; solche Gegenecken sollen immer denselben Index erhalten. Die Punkte $A$ und die ersten Pro-

Fig. 61 .

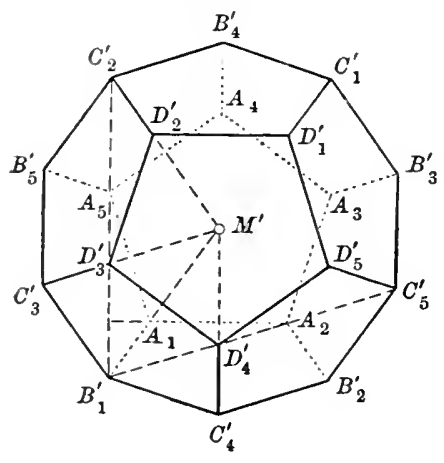
jektionen der Punkte $D$ bilden zusammen die Ecken eines regelmäbigen Zehnecks, und ebenso sind die Punkte $B^{\prime}$ und $C^{\prime}$ die Ecken eines zweiten regelmäßigen Zelnnecks, das mit dem ersten parallel und konzentrisch ist. Wir bezeichnen die Radien der umgeschriebenen Kreise für das erste und zweite Zehneck bzw. mit $r_{1}$ und $r_{2}$, die Seite des ersten mit $s$.

Betrachten wir die Grundfläche $A_{1} \ldots A_{5}$ als gegeben, so ist zur Bestimmung des Grundrisses des Körpers ein Eckpunkt des zweiten Zehnecks, z.B. $B_{1}^{\prime}$, erforderlich. Drehen wir nun das Fünfeck $A_{1} B_{1} C_{4} B_{2} A_{2}$ um $A_{1} A_{2}$, bis es nit der Cirundfläche zusammenfällt, so gelangt $\boldsymbol{B}_{1}$ nach $\boldsymbol{A}_{5}$; $B_{1}^{\prime}$ liegt folglich auf dem Lote von $A_{5}$ auf $A_{1} A_{2}$, und ebenso ist $A_{2} B_{1}^{\prime} \perp A_{1} A_{5}$. - Noch genauer erhalten wir $l_{1}^{\prime}$ durch folgende Überlegung: Die vier Punkte $B_{1}^{\prime}, D_{3}^{\prime}, A_{5}, C_{2}^{\prime}$ liegen auf einer Parallelen zu $M^{\prime} D_{4}^{\prime}$ und die Punkte $B_{1}^{\prime}, D_{4}^{\prime}, A_{2}$, $C_{5}^{\prime}$ auf einer Parallelen zu $M L^{\prime} l_{3}^{\prime}$. Das Viereck $M L^{\prime} D_{3}^{\prime} I_{1}^{\prime} I_{4}^{\prime}$ ist folglich ein Rhombus, also ist $D_{3}^{\prime} B_{1}^{\prime}=r_{1}$, und die Strecke $M^{\prime} B_{1}^{\prime}$ wird von $D_{3}^{\prime} D_{4}^{\prime}$ senkrecht halbiert. Ferner ist $A_{1} D_{3}^{\prime} \| M^{\prime} C_{2}^{\prime}$, also $\Delta B_{1}^{\prime} A_{1} I_{3}^{\prime} \sim \Delta B_{1}^{\prime} M^{\prime} C_{2}^{\prime}$, mithin $\Delta B_{1}^{\prime} A_{1} D_{3}^{\prime}$ gleichschenklig und $A_{1} B_{1}^{\prime}=s$. Hieraus folgt noch $r_{2}=r_{1}+s$.

Un den Aufrib za konstruieren, branchen wir nur noch die Entfernungen der Punkte $B$ und $C$ von $\Pi_{1}$ zu bestimmen. Bezeichnen wir sie bzw. mit $h$ und $i$, so ist der erste 'Tafelabstand der Punkte $I)=h+i$. - Da die Bildstrecken $A_{5} C_{2}^{\prime}$ und $A_{5} B_{1}^{\prime}$ auf $A_{5} A_{3}$ senk- 
recht stehen, so sind nach $A_{1}$ t. 30 auch die (Originalstrecken $A_{5} C_{2}$ and $A_{5} B_{1}$ senkrecht anf $A_{5} A_{3}$. Wir schließen hieraus allgemein, da $B$ von den sechs Diagonalen der Seitentlächen, die von einer Ecke ausgehen, je zwei aufeinauder senkrecht sind, die durch eine Diagonale getrennt werden. Daher steht auch $A_{5} C_{2} \perp A_{5} B_{1}$, und da beide Strecken einander gleich sind, so ist $\triangle A_{5} B_{1}^{\prime} B_{1}^{\prime} \sim \Delta C_{2}^{\prime} A_{5} C_{2}^{\prime}$, folglich $B_{1} B_{1}^{\prime}=A_{5} C_{2}^{\prime}$ und $C_{2} C_{2}^{\prime}=A_{5} B_{1}^{\prime}$. Nun ist aber $A_{5} C_{2}^{\prime}=I_{3}^{\prime} B_{1}^{\prime}=r_{1}$ und $A_{5} B_{1}^{\prime}$ $=A_{5} I_{3}^{\prime}+D_{3}^{\prime} B_{1}^{\prime}=s+r_{1}=r_{2}$, mithin ergibt sich $h=r_{1}$ und $i=r_{2}$.

Man konstruiere von dem dargestellten Zwölfflach auch die schiefe Projektion auf die $\Pi_{2}$.

\section{Schnitt eines Vielflachs mit einer Ebene und mit einer}

Geraden.

56. Um den Schnitt eines Vielflachs mit einer Ebene zu konstruieren, kann man durch wiederholte Anwendung des in Art. 28 entwickelten Verfahrens die Schnittpunkte seimer Kanten mit der Ebene ermitteln; dabei kommen aber nur solche Schnittpunkte in Betracht, die sich innerhalb der begrenzten Flächen befinden. Hierdurch erhält man alle Eckpunkte der gesuchten Schnittfigur, und dann hat man schließlich die Punkte, die in derselben Fläche liegen, zu verbinden (Kantenverfahren).

Besonders einfach gestaltet sich die Lösung, wenn die schneidende Ebene auf einer der Projektionstafeln senkrecht steht; dann ist nämlich die betreffende Projektion der Ebene, also auch der Schnittfigur, eine Gerade, und damit sind die Schuittpunkte der überhaupt in Frage kommeliden Kanten uumittelbar gegeben. Deshalb empfiehlt sich im allgemeinen Fall die Ein führung einer neuen Projektionsebene, senkrecht zur schneidenden Ebene und zu einer der alten Tafeln, d. h. senklecht zu einer Spur der gegebenen Ebene (vgl. Art. 46).

Aufgabe. Den Schnitt der Ebene $E\left(e_{1} f_{2}\right)$ mit einer Pyramide zu konstruieren, deren Grundfläche $A B C \ldots$ in $\Pi_{1}$ liegt (Fig. 62). Wir ermitteln die Schnittfigur $A_{1} B_{1} C_{1} \ldots$ mit Hilfe einer Seitenrißebene $\Pi_{3} \perp e_{1}$, ziehen also $y \perp e_{1}$ und zeichnen in der Umlegung in $\Pi_{1}$ die

Fig. 62.

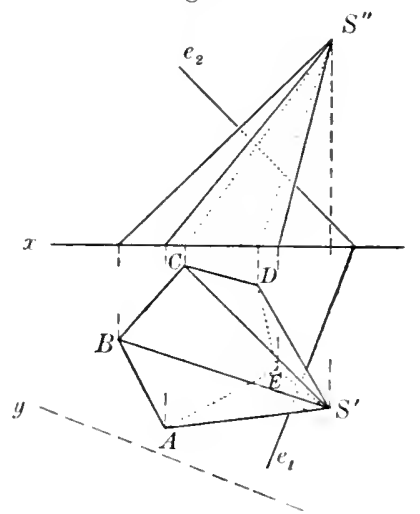
dritte Spur $e_{3}$ von E (Art. 46) sowie den

Seitenriß der Pyramide. Von der Spitze $S$ ergibt sich nach Art. 11 die dritte Projektion $S^{\prime \prime \prime}$ mittels $S^{\prime} S^{\prime \prime \prime} \perp y$ und $S_{y} S^{\prime \prime \prime}=S, S^{\prime \prime}$. Dann schneidet $\iota_{3}$ die Gerade $S^{\prime \prime \prime} A^{\prime \prime \prime}$ im Punkte $A_{1}^{\prime \prime}$, und diesem entsprechen auf $S^{\prime} A$ und $S^{\prime \prime} A^{\prime \prime}$ die Punkte $A_{1}^{\prime}$ und $A_{1}^{\prime \prime}$.

Die Grundkante $A B$ und die Seite $A_{1} B_{1}$ der Schnittigur treffen sich a uf $e_{1}$, denn die drei Schnittlinien der drei Ebenen $S A B, \Pi_{1}$ und $E$ gehen durch einen Punkt. 
Die wahre Größe der Schnittfigur wird durch l'mlegung in $\Pi_{1}$ gefunden (Art. 46).

Um das Netz der Pyramide zu konstruieren, ermittelt man zunächst die wahren Längen der Seitenkanten, z. B. der Kante SA als Hypotenuse eines rechtwinkligen Dreiecks mit den Katheten $S^{\prime} A$ und $S^{\prime \prime} S_{x}$ (oder $S^{\prime \prime \prime} S_{y}$ ). Dann zeichnet man das Dreieck $S A I$ a as soinen drei Seiten, heftet an $S B$ das Dreieck $S B C$ usw. Man kann aber auch jede Seitenfläche einzeln um ihre Grundkante in $\Pi_{1}$ umlegen (Art. 39).

Die Konstruktion des Netzes der abgestumpften Iyramide erfordert noch die Bestimmung der wahren Langen der Kantenabschnitte $A A_{1}, B B_{1} \ldots$ Nun verlalten sicb nach Art. 1 die Abschnitte auf der wabren Läuge einer Kante wie die entsprechenden Abschnitte auf einer ilurer Projektionen: hatte man also vorher die wahre Lünge von $S A$ durch Abtragen der Strecke $S^{\prime} A$ anf $x$ ermittelt, so findet man auf ihr die Länge von $A A_{1}$ mittels einer Parallelen zu $x$ durch den Punkt $A_{1}^{\prime \prime}$. Noch genauer bestimmt man die Kantenabschnitte mittels des Seitenrisses: Macht man auf $y$ die Streeke $S_{y} A_{0}=S^{\prime} A$ und zieht $A_{1}^{\prime \prime \prime} A_{10} \| y$ bis $S^{\prime \prime \prime} A_{0}$, so ist $A A_{1}=A_{0} A_{10}$.

Kontrolle: Die Strecke $A_{1} B_{1}$ des Netzes muB gleich sein der entsprechenden Seite der ungelegten Schnittfigur.

57. Aufgabe. Von einem schiefen Prisma mit der in $\Pi_{1}$ liegenden Grundfläche $A_{1} B_{1} C_{1} \ldots$ und der Deekfläche $A_{2} B_{2} C_{2} \ldots$ das Netz zu konstruiereu (Fig. (i3). Die Seitenflächen sind Parallelo-

Fig. 63 .

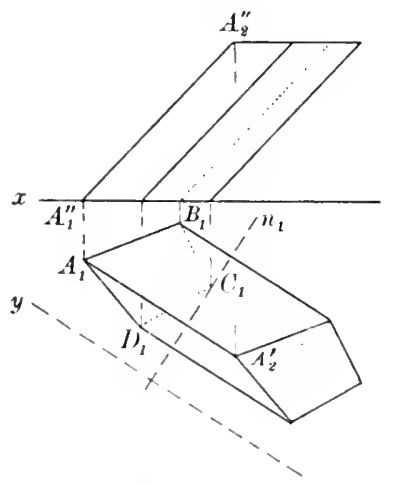
gramme, deren wahre Größe aus je zwei Seiten und einer Diagonale oder durch Umlegung in $\Pi_{1}$ leicht gefunden wird (vgl. Art. 19 und 39). Noch vorteilhafter ist in der Regel die Anwendung eines Normalschnittes: Sehneiden wir das Prisma senkrecht zu den Seitenkanten mit einer Ebene N, so erhalten wir ein Vieleck $A_{3} B_{3} C_{3} \ldots$, dessen Seiten auf den Kanten senkrecht stehen; dieser Schnitt verwandelt sich daher beim Abwickeln in eine Gerade.

Die Ausführung der Konstruktion gestaltet sich in folgender Weise: Wir ziehen die Spur $n_{1}$ von $N \perp A_{1} A_{2}^{\prime}$, am zweckmäbigsten durch eine Eeke der Grundfläche, etwa durch $C_{1}$, und zeichnen den Seitenrib auf eine $\Pi_{3} \perp n_{1}$ mittels $y \| A_{1} A_{2}^{\prime}, A_{1} A_{1}^{\prime \prime \prime}$ und $A_{2}^{\prime} A_{2}^{\prime \prime \prime} \perp y$, Abstand $A_{2}^{\prime \prime \prime} y=$ Abstand $A_{2}^{\prime \prime} x$ usw. Die Spur $n_{3}$ von $\mathrm{N}$ geht durch den Schnittpunkt $C_{1}^{\prime \prime \prime}$ von $n_{1}$ mit $y$, und zwar $\perp A_{1}^{\prime \prime \prime} A_{2}^{\prime \prime \prime}$; sie bestimmt auf den dritten Projektionen der Seitenkanten die Punkte $A_{3}^{\prime \prime \prime}, B_{3}^{\prime \prime \prime}$... Wir ermitteln ferner die wahre Größe des Normalschnitts durch Umlegung in $\Pi_{1}$; dabei fällt $A_{3}$ mach $A_{3}^{\prime \prime}$ auf $A_{1} A_{2}^{\prime}$, und es ist $A_{\text {bstand }} A_{3}^{\prime \prime} n_{1}=A_{3}^{\prime \prime \prime} C_{1}^{\prime \prime \prime}$. Nach diesen Vorbereitungen ziehen wir eine beliebige Gerade als Abwickelung des Normalschnittes und macben auf ihr die Strecke $A_{3} B_{3}$ $=A_{3}^{9} B_{3}^{\prime \prime}$ nsw. Da die Seitenkanten zu $\Pi_{3}$ parallel sind, so erscheinen 
sie im Seitenrib in wahrer Gröbe; im Netz ist also $A_{3} A_{1} \perp A_{3} B_{3}$ und $=A_{3}^{\prime \prime \prime} A_{1}^{\prime \prime \prime}$.

Kontrolle: Die im Netz und im Grundrib mit $A_{1} B_{1}$ bezeichneten Strecken müssen einander gleich sein.

58. Zur Ermittelung des ebenen Schnittes eines Vielflachs kann außer den bisher entwickelten Methoden, die stets zum Ziele führen, in gewissen Fällen auch ein Fläcbenverfahren benutzt werden, das unmittelbar die Schnittlinien der Flachen des hörpers mit der Ebene, also statt der Ecken sofort die Seiten der Schnittfigur liefert.

Aufgabe. Den Schnitt der Ebene $\mathbf{E}\left(e_{1} e_{2}\right)$ miteinemschiefen Prismazu konstruieren, dessen Grundfläche $A_{1} B_{1} C_{1} \ldots$ in $\Pi_{1}$ liegt (Fig. 64). Die Deckfläche $A_{2} B_{2} C_{2} \ldots$ befindet $\operatorname{sich}$ in einer borizontalen Ebene $\Sigma$, und cliese schneidet $E$ in einer Hauptlinie $m \| e_{1}$. Dann kennt man z. B. von der Schnittlinie der Ebenen $A_{1} B_{1} B_{2} A_{2}$ und $E$ zwei Punkte, nämlich in $\Pi_{1}$ den Punkt $V=A_{1} B_{1} \times e_{1}$ und in $\Sigma$ den Punkt $W=A_{2} B_{2} \times m$; die Gerade $V W$ liefert daher eine Seite $A_{3} B_{3}$ der Schnittfigur.

Fig. 64 .

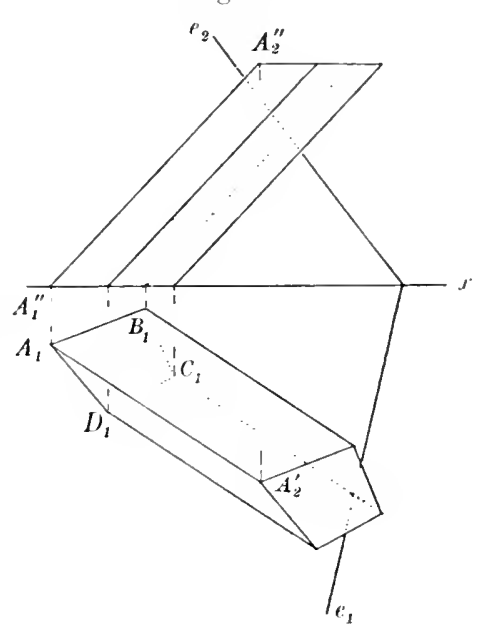

Ebenso findet man den ebenen Schnitt einer auf der $\Pi_{1}$ stehenden Pyramide mit Hilfe einer horizontalen Ebene $\Sigma$. die man zweckmäBir durch die Spitze $S$ legt: Die erweiterte Seitenfläche $S A B$ schneidet $\Sigma$ in einer Parallelen zu $A B$, und diese trifft die Hauptlinie $m$ wie vorhin in einem Punkte $W$ der Schnittlinie der Ebenen $\mathrm{E}$ und $S A B$.

59. Um die Schnittpunkte einer Geraden mit einem Vielflach zu bestimmen, legt man durch die Gerade eine Hilfsebene und ermittelt ihren Schnitt mit dem Vielflach, sowie die Schnittpunkte der Geraden mit dieser Schnittfigur. Die Hilfsebene ist naturgemäß so zu wählen, daß ihr Schnitt mit dem Vielflach möglichst leicht konstruiert werden kann: am geeignetsten sind daher in der Regel die projizierenden Ebenen der Geraden.

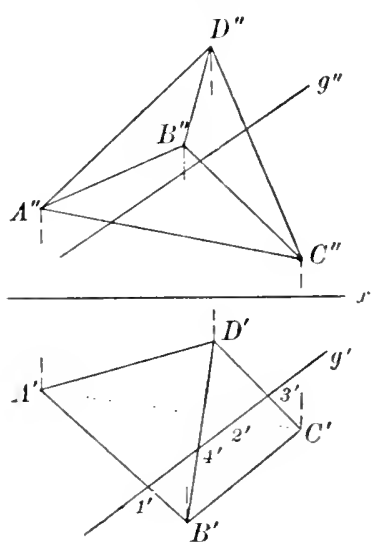

Aufgabe. Die Schnittpunkte $P, Q$ der Geraden $g$ mit dem Tetraeder $A B C D$ zu ermitteln (Fig. 65). Die erste projizierende Ebene von $g$ schneidet das Tetraeder in dem 
Viereck 1234 , dessen Grundrib bekannt ist, so daB sein Aufrib sofort konstruiert werden kann. Die Gerade $g^{\prime \prime}$ trifft $1^{\prime \prime} 2^{\prime \prime}$ in $P^{\prime \prime}, 3^{\prime \prime} 4^{\prime \prime}$ in $Q^{\prime \prime}$. Der Punkt $I^{\prime}$ liegt daher in der Fläche $A B C$ und $Q$ in der Fläche $B C D$. Der anßerlalb des Tetraeder's liegende 'Teil von $g$ ist im Aufriß von $P^{\prime}$ an sichtbar, im Grundriß dagegen nicht, weil die Fläche $A B C$ zwar im Aufrib, aber nicht im Grundrib geselen wird.

In Fig. 65 ist die Gerade $B^{\prime \prime} D^{\prime \prime}$ nahezu senkrecht auf $x$; um iu diesem Falle den Punkt $4^{\prime \prime}$ möglichst genau zu bestimmen, ziehe man im Dreieck $A B D$ die Hilfslinie $4 J \| B A$ bis $A D$, also $t^{\prime} J^{\prime} \| B^{\prime} A^{\prime}$, und irmittle . ${ }^{\prime \prime} 4^{\prime \prime}$.

Noch einfacher gestaltet sich in vorliegenden Falle die Lösung mittels der $z$ weiten projizierenden Ebene von $g$, weil diese das Tetraeder nur in einem Dreieck schneidet.

Durchdringung zweier Vielflache.

80. Zwei Vielflache durchschneiden sich entweder in einem oder in mehreren (windschiefen oder ebenen) Vielecken. Im ersten Falle sagt man, sie dringen ineinander ein (schneiden sich gegenseitig an), im zweiten spricht man von einer vollständigen Durchdringung (Durchbohrung) des einen Vielflachs durch das andere.

Um die Durchdringungsfigur zu konstruieren, bestimmt man in der Regel zunächst ihre Ecken als die Schnittpunkte der unverlängerten Kanten des einen Vielflachs mit den unerweiterten Flächen des anderen und verbindet dann immer zwei Eckpunkte miteinander, die in jedem Vielflach derselben Fläche angehören (Kantenverfahren). In gewissen Fällen findet man aber auch unmittelbar die Seiten der gesuchten Figur als die Schnittlinien der Fläcben des einen Vielflachs mit den Flächen des anderen (Flächenverfahren).

Beim Kantenverfahren legt man durch die einzelnen Kanten geeignete Hilfsebenen und ermittelt für jede solche Ebene ihren Schnitt mit dem anderen Vielflach und hierauf die Punkte, in denen die betreffende Kante das so erhaltene Vieleck schneidet (Art. 59). Als Hilfsebenen dienen im allgemeinen die projizierenden Ebenen der Kanten (vgl. jedoch Art. 62).

61. Aufgabe. Die Durchdringung des Tetraeders $A B C D$ mit dem dreiseitigen Prisma $E F$ (i $H J K$ zu konstruieren(Fig. 66). Wir lösen die Aufgabe nach dem Kantenverfabren mittels projizierender Ebeneu durch die einzelnen Kanten (Art. 59). Um z. B. die Schnittpunkte der Tetraederkante $A B$ mit dem Prisina zu bestimmen, benutzen wir die zweite projizierende Ebene ron A B. Diese schneidet die Prismakanten $E H, F, I$. G $K$ bzw. in den Punkten $1,2,3\left(1^{\prime \prime}=A^{\prime \prime} B^{\prime \prime}>E^{\prime \prime} I^{\prime \prime}\right)$. Die Strecke $A^{\prime} B^{\prime}$ hat mit den Seiten $2^{\prime} 3^{\prime}$ und $3^{\prime} 1^{\prime}$ des Dreiecks $1^{\prime} 2^{\prime} 3^{\prime}$ die Punkte $L^{\prime}$ und $M^{\prime}$ gemein; die Kante $A B$ durchstöBt also die Prismaflächen $F G K J$ und $G E / K$ in $L$, nnd $M$. - In derselben Weise verfahren wir der Reihe nach mit allen Tetraeder- und Prismakanten, diejenigen ausgenommen, bei dewen die Figur ohne weiteres zeigt, dab sie ganz außerhalb des anderen Vielflachs liegen. 
Wir haben schlieBlich unter den gefundenen Schnittpunkten immer je zwei, die auf derselben Tetraederfläche und zugleich auf derselben Prismafläche liegen, zu einer Seite der Schnittfigur zu verbinden. Dazu bedienen wir uns zweckmäßig einer Tabelle, in der wir neben jedem Sclınittpunkte die Flächen eintragen, in denen er sich befindet:

\begin{tabular}{c|c|c}
\hline Punkte & Prismafl̈chen & Tetraederflächen \\
\hline$L$ & FGKJ & $A B C, A B D$ \\
$M$ & $G E H K$ & $A B C, A B D$ \\
& usw.
\end{tabular}

Bei diesem Verfahren kann es sich ereignen, dab die projizierende Ebene, die wir durch eine bestimmte hante (z. B. FJ) legen, das andere Vielflach in einem Vieleck schneidet, das von der Kante gar nicht getroffen wird; dann zeigt sich erst hierdurch, daß die betrachtete Kante überhaupt keinen Eckpunkt der Durchdringungsfigur enthält. Um solche erfolglose Versuche von vornherein $\mathrm{zu}$ vermeiden, können wir: nachdem für eine Kante, etwa $A B$, wie vorhin die Schnittpunkte $L$ und $M$ bestimmt sind, in folgender Weise vorgehen. Ia der Punkt $M$ in der Prismafläche GEHK und in der Tetraederfläche $A B C$ liegt, so beginnt in ihm die Schnittlinie dieser Flächen, und wir finden sie, indem wir noch eine Kante der einen Fläche mit der anderen zum Schnitt bringen. Benutzen wir hierzu z. B. die Kante $E H$ und konstruieren nach Art. 28 ihren Schnittpunkt $W$ mit der Ebene $A B C$, so ergibt sich $M W$ als Schnittlinie beider Flächen. Diese gehört aber der Durchdringungsfigur nur so weit an,

Fig. 66.
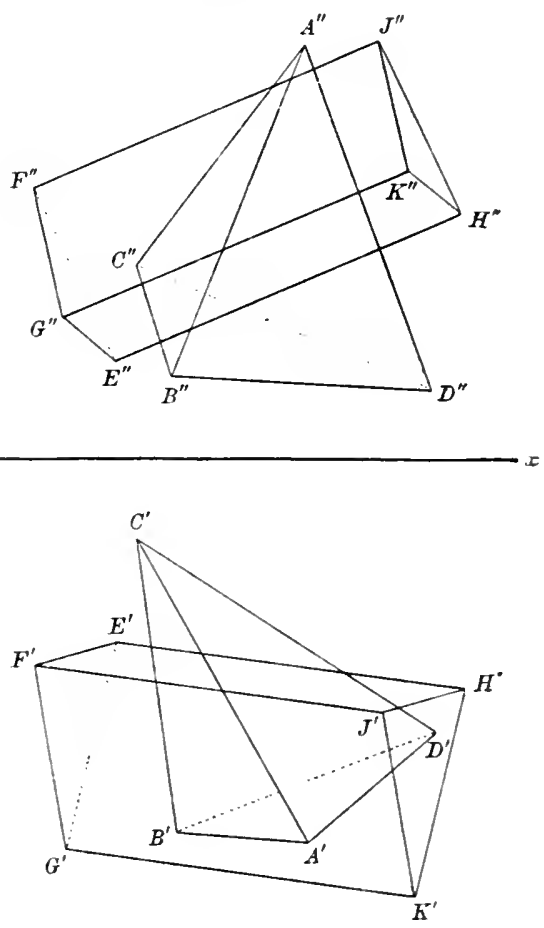
als sie sich innerhalb des Dreiecks $A B C$ befindet, also bis zu ihrem Schnittpunkte $S$ mit der Kante $B C$. Da $B C$ auch im Dreieck $B C D$ liegt, so beginnt in $S$ die Schnittlinie dieser Fläche mit $G E H K$, von der wir wieder einen zweiten Punkt ermitteln, usw. Auf diese Weise erhalten wir die Eckpunkte der Durch- 
dringungsfigur sofort in der richtigen Reihenfolge, so dab die Anlegung einer Tabelle ïberflïssig wird.

Die Projektion einer Seite der I)urchdringungsfigur ist als sichtbar anszuziehen, wenn beide Flächen, deren schnittlinie sie ist, in der betreffenden Projektion gesehen werdeu.

Kontrollen: Die beiden Schnittlinien einer Fläche des einen Vielflachs mit zwei in einer hante zusammenhängenden Flächen des anderen treffen sich immer anf dieser Kante.

62. Handelt es sich $u m$ die Durchdringung zweier Pyramiden, so können wir die Konstruktion nach dem Kantenverfahren dadurch vereinfachen, daf wir an Stelle der bisher benutzten projizierenten Ebenen zweckmäbiger gewählte Hilfsebenen verwenden. Bei einer

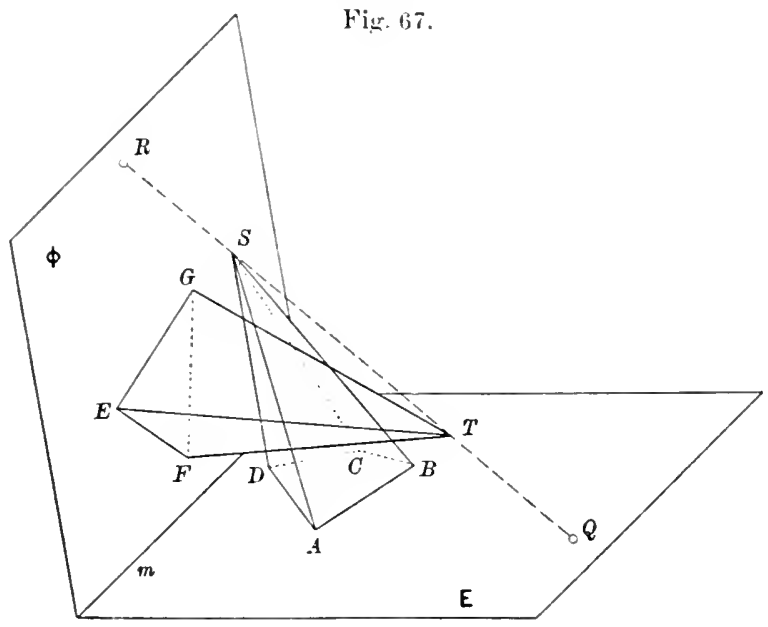

Pyramide werden nämlich die einfachsten Schnitte durch solche Ebenen erzeugt, die durch die Spitze gehen, denn diese schneiden die Mantelfläche in Geraden aus der Spitze. Um daher die Schnittpunkte der Kanten der einen Pyramide mit der anderen zu konstruieren, legen wir durch jede Kante eine Hilfsebene, die zugleich die spitze der anderen Pyramide enthält, d. h. Wir benutzen Hilfsebenen durch die Verbindungslinie beider spitzen.

Sind $S$ und $T$ die Spitzen der beiden Pyramiden, $A B C D$ und $E F$ G $r$ bzw. die zugehörigen Grundflächen in den Ebenen $E$ und $\Phi$, so bestimmen wir zunächst die Schnittlinie $m$ dieser Ebenen, sowie die Schnittpunkte $Q$ und $l i$ der Geraden $S T$ mit $E$ und $\Phi$ (Fig. (iT). Dann erhalten wir z. B. die Schnittpunkte $A_{1}$ und $A_{2}$ der Kante $S A$ mit der anderen Pyramide, indem wir durch $S A$ und $S T$ eine Hilfsebene legen. Diese schneidet $\mathrm{E}$ in $Q A, m$ in $\mathfrak{H}$ (auf $Q A$ ), $\Phi$ in $\mathbb{Z} R$, das Dreieck

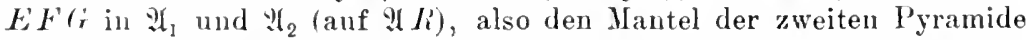
in den Geraden $\mathfrak{I}_{1} T$ und $\mathfrak{H}_{2} T$, die auf $S A$ die Punkte $A_{1}$ und $A_{2}$ bestimmen. Haben wir in derselben Weise alle übrigen Eckpunkte der Durchdringungsfigur gefunden, so erkennen wir ibre Reihenfolge ohne 
Benutzung einer Tabelle, indem wir von $A$ und $\mathfrak{A}_{1}$ aus beide Grundflächen derartig umfahren, dals die in ihnen gleichzeitig erreichten Punkte immer in derselben Hilfsebene liegen.

Rückt der Punkt $T$ in unendliche Entfernung, so verwandelt sich die zugehörige Pyramide in ein Prisma. Dann bleibt die vorige Konstruktion ungeändert, nur werden die Geraden $S T, \mathfrak{A}_{1} T, \mathfrak{A}_{2} T$. . parallel zu den Seiteukanten des Prismas.

Nach ganz demselben Verfahren ermitteln wir endlich auch die Durchdringung zweier Prismen, indem wir durch die Seitenkanten jedes Prismas Hilfsebenen legen parallel zu denen des anderen (vgl. Art. 24, Schluß). Alle diese Ebenen schneiden E und $\Phi$ in parallelen Geraden.

83. Aufgabe. Die Durchdringung eines Prismas miteiner Pyramide zu konstruieren, wenn die zugehörigen Grundflächen $A B C D$ und $K L M$ in $\Pi_{1}$ liegen (Fig. (i8). Erste Lösung (Kantenverfahren): Nach Art. 62 ziehen wir durch die Spitze $S$ der Pyramide eine Parallele zu den Seitenkanten des Prismas bis zu ihrem Schnittpunkte $Q$ mit $\Pi_{1}$. Um dann die Schnittpunkte $A_{1}$ und $A_{2}$ der durch $A$ gehenden Prismakante mit der Pyramide zu ermitteln, legen wir durch diese Kante und durch $S Q$ eine Hilfsebene: ihre Grundrißspur $Q A$ schneidet $K I$ in $\mathfrak{A}_{1}, I, \mathbb{H}$ in $\mathfrak{H}_{2}$ usw.

Die Netze der beiden Körper werden wie in Art. 56 und 57 konstruiert. Um den Eckpunkt $A_{1}$ der Durchdringungsfigur im Netz der Pyramide anzugeben, übertragen wir zunächst den Punkt $\mathfrak{A}_{1}$, ziehen dann die Gerade $S \mathfrak{A}_{1}$ und machen auf ihr $\mathfrak{A}_{1} A_{1}$ gleich der wahren Länge dieser Strecke.

Zweite Lösung (Flächenverfahren, vgl. Art. 58): Eine horizontale Ebene durch $S$ schneidet das Prisma Fig. 68.

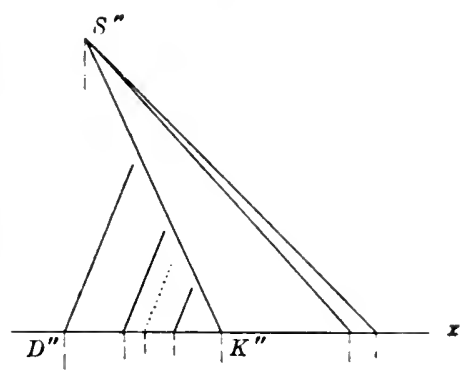
in einem Viereck $E F G H$, das zu

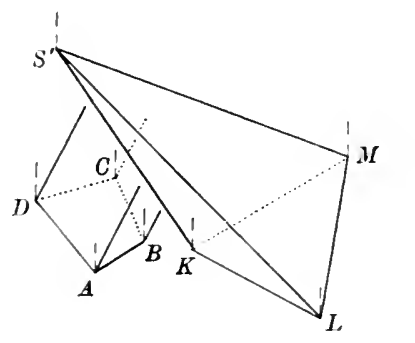

$A B C D$ parallel und kongruent ist, und die erweiterten Seitenflächen der Pyramile in drei durch $S$ gehenden Geraden $p\|K L, q\| L, I$, $r \| M K$. Um z. B. die Schnittlinie der Flächen $A B F E$ und $S K L$ zu ermitteln, bestimmen wir die Punkte $T=A B \times K L$ und $U=E F \cdot p$; dann ist $T U$ die gesuchte Schnittlinie, die aber als Seite der Durchdringungsfigur nur so weit in Betracht kommt, als sie innerhalb der begrenzten Seitenflächen liegt, also von ihrem Schnittpunkte $A_{1}$ mit $A E$ bis $K_{1}$ auf $S K$. In $A_{1}$ wird sich die Schnittlinie der Flächen $A D H E$ und $S K L$ anschließen usw.

Soll die Seite $A_{1} K_{1}$ der Durchdringungsfigur in das Netz der Pyramide eingetragen werden, so benutzen wir wieder die Punkte $T$ auf $K L$ und $U$ auf $p$, ziehen also in Netz $S U \| K L$ und $=S^{\prime} U^{\prime}$. 


\section{Schatteukonstruktionen.}

64. Um die Anscbaulichkeit der Abbildung zu erhöhen, denken wir uns die dargestellten Objekte aus einem endlichen oder unendlich fernen Punkte $L$ beleuchtet. Während in bezug auf $L$ als I'rojektionszentrum die Oberfläche jedes undurchsichtigen Körpers in einen sichtbaren und einen unsichtbaren Teil zerfällt, die durch den wahren Imrib getrennt werden, unterscheiden wir für $I$, als Lichtquelle diese beiden Oberflächenteile als den beleuchteten und den im Eigen-oder Selbstsclutten befindlichen Teil und bezeichnen ihre Trennungslinie, in deren Punkten die Lichtstrahlen die Oberfläche streifen (oder ber ühren), als Eigen-oder Selbstschattengrenze (Lichtgrenze). Die streifenden Lichtstrahlen umschließen hinter dem Körper seinen Schattenraum. Jede Oberfläche, die in diesen Raum hineinreicht, empfängt vom Körper einen Schlagschatten, dessen Grenzlinie von jenen streifenden Lichtstrahlen ausgeschnitten wird. Die Schlagschattengrenze ist also die Projektion der Eigenschattengrenze aus $L$ und geometrisch dasselbe, wie ler scheinbare Umriß des Körpers fül $L$ als Projektionszentrum.

Wenn nicht ausdrücklich das Gegenteil bemerkt ist, werden wir im folgenden immer voraussetzen, der Punkt $L$ sei unendlich fern. Wil wählen ferner die parallelen Lichtstrahlen immer so, daß sie von links oben und vorn wach rechts unten und hinten gerichtet sind, und zwar zumeist parallel zur Diagonale eines Würfels, dessen Flächen zu den Projektiousebenen parallel oder senkrecht sind. Diese namentlich in technischen Zeichnungen bevorzugte Lichtrichtung, deren Projektionen gegen $x$ unter $45^{0}$ geneigt sind, soll kurz als „lichtung der Würfeldiagonale" bezeichnet werden.

65. Aufgabe. Bei gegebener Lichtrichtung $l\left(l^{\prime}, l^{\prime \prime}\right)$ den schlagschatten zu bestimmen, den der Punkt $P^{\prime}\left(P^{\prime}, P^{\prime \prime}\right)$ auf eine der Projektionsebenen wirft. Der Schlagschatten von $P$ auf

Fig. 69. $\quad \Pi_{1}$ oder $\Pi_{2}$ ist der erste oder zweite Spurpunkt des

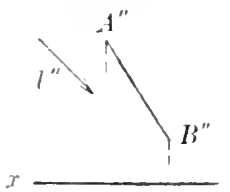
lurch $P$ gehenden Lichtstrahls (Art. 17); wir bezeichnen ihn fortan mit $I_{h}^{\prime}$ oder mit $P_{v}$. Da die Projektiousebenen als unbegrenzt und undurchsichtig vorausgesetzt werden, so werfen zufolge der Annahme, die wir in Art. 64 über die Lichtrichtung gemacht haben, nur die im ersten Quadranten liegenden Punkte Schatten, und zwar entweder auf die $+\Pi_{1}$ oder auf

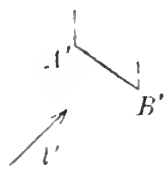
die $+\Pi_{2}$. Für Licht in der Richtung der IVürfelliagonale ist $P_{h} I_{v}^{\prime} \| x$.

86. Aufgabe. Den Schlagschatten der Strecke $A B$ auf die Projektionsebenen zu konstruieren. Man ermittle von den Endpunkten $A$ und $B$ den Schatten $A_{h}$ und $B_{h}$ auf $\Pi_{1}$. Liegt, wie in Fig. $69, A_{h}$ oberhalb, $B_{h}$ unterhalb $x$ und schneidet $A_{h} B_{h} x$ in $C$, so ist der Schatten von $A l$, die gebrochene Linie $A_{v} C B_{h}$.

Ist $A B \| \Pi_{1}$, so wird $A_{h} B_{h} \# A B$. Steht $A B \perp \Pi_{1}$, so ist $A_{h} B_{h} \| \gamma^{\prime}$. 
67. Um zu entscheiden, ob von einer ebenen Figur, etwa dem in Fig. 70 dargestellten Dreieck $A B C$, im Grundriß die beleuchtete oder die im Eigenschatten befindliche Seite gesehen wird, schneiden wir die Figur mit einer Ebene, die zur ersten projizierenden Ebene des Lichtstrahls $l$ parallel ist, in einer Geraden $D E\left(D^{\prime} E^{\prime} \| l^{\prime}\right)$ Dagegen zeigt der Aufriß, daß im vorliegenden Falle die in der Hilfsebene verlaufenden ersten projizierenden Strahlen und die in ihr liegenden Lichtstrablen entgegengesetzte Seiten vou $D E$ treffen; demnach ist die im Grundriß sichtbare Seite der Ebene $A B C$ nicht beleuchtet.

Da die Dreiecke $A^{\prime} B^{\prime} C^{\prime}$ und $A^{\prime \prime} B^{\prime \prime} C^{\prime \prime}$ entgegengesetzten Sinnes sind, so sieht man im Aufriß die beleuchtete Seite der Ebene (vgl. Art. 21).

Der Grundrib einer ebenen Figur und ihr Schatten auf die $\Pi_{1}$ sind perspektir affin mit der Grundrißspur der Ebene der Figur als Affinitätsachse.

68. Aufgabe. Den Schlagschatten des Fig. 70 .
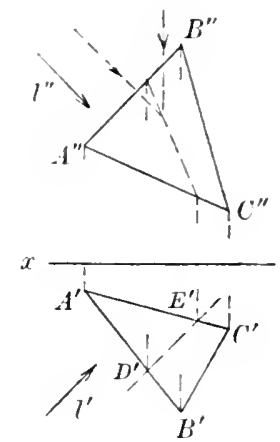
Prismas $A B C D E F$ auf die Projektionsebenen, sowie seinen Eigenschatten zu bestimmen (Fig. 71 ). Wir konstruieren zunächst den vollständigen Schlagschatten des Prismas auf die $\Pi_{1}$, so wie er sich gestalten würde, wenn die $\Pi_{2}$ durchsichtig wäre. Haben wir von den durch $A$. $B, C, D$ gezogenen Lichtstrahlen die ersten Spurpunkte $A_{h} \ldots$ ermittelt, so ergeben sich $E_{h}$ und $F_{h}$ sofort aus der Bemerkung, daß $B_{h} E_{h}$ und $C_{h} F_{h} \# A_{h} D_{h}$ sind. Denken wir uns nun die Schatten aller Eckpunkte verbunden, die auf dem Prisma selbst durch Kanten verbunden sind, so erhalten wir als Schlagschattengrenze das Fünfeck $A_{h} C_{h} B_{h} E_{h} D_{h}$, das alle übrigen Verbindungslinien einschließt, und diesem entspricht auf dem Prisma die Eigenschattengrenze $A C B E D$. Daß übrigens die Punkte $C$ und $D$ der Eigenschattengrenze angehören, ist ohne weiteres klar, weil in $C^{\prime}$ und $D^{\prime}$ der erste scheinbare Umriß von je einer Parallelen zu $l^{\prime}$ gestreift wird, und das Entsprechende gilt im Aufriß von $A$ und $E$. - Da der Kantenzug $A C B E D A$ die beleuchteten Prismaflächen von den im Eigenschatten Fig. 71.
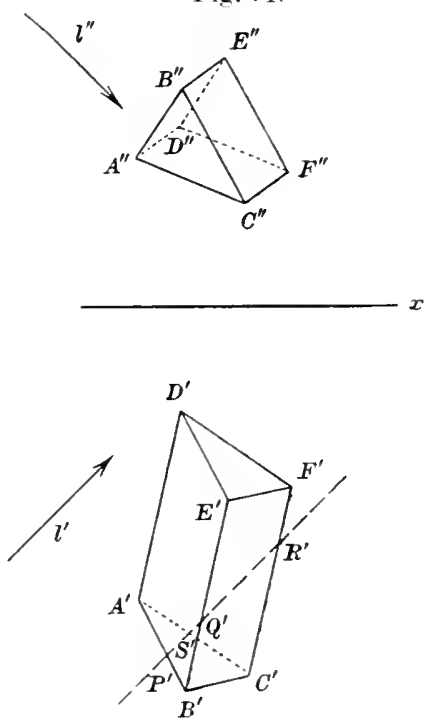
befindlichen trennt, so ergibt sich aus der Anschauung; daß nur die Flächen $A B C$ und $A B E D$ beleuchtet sind. Davon überzeugen wir uns auch, indem wir das Prisma mit einer Hilfsebene schneiden, die zur ersten projizierenden Ebene von 7 
parallel ist. Die so erhaltene Schuittigur I'QRS wird - wie der Aufrib zeigt - von den durch $Q$ und $S$ gehenden Lichtstrahlen gestreift, und die in $A B C$ und $A B E ; D$ liegenden Seiten $S P$ und $I^{\prime} Q$ sind dem Lichte zugewendet. Oder: Der durch den Schnittpunkt von $A_{h} B_{h}$ und $C_{h} F_{h}$ gehende Lichtstrahl trifft zuerst die Fante $A B$ und dann die Fante $C F$, folglich liegt $A B$ im beleuchteten Teile der OberHäche des Körpers und (' $F$ ' im Eigenschatten.

Die Punkte $I)$ und $E$ werfen ihren Schatten in Wirklichkeit nicht nach $D_{h}$ und $E_{h}$, sondern nach $D_{v}$ und $E_{v}$; die Schlagschattengrenze des Prismas tritt also in den Schnittpunkten $M$ und $N$ von $A_{h} D_{h}$ und $B_{h} E_{h}$ mit $x$ anf die Ebene $\Pi_{2}$ über. Die Figuren $M D_{h} E_{h} N$ und $M D_{v} \mathrm{E}_{v} N$ sind perspektiv affin mit $x$ als Affinitätsachse.

69. Um den Schlagschatten einer Figur auf eine andere F’igur zu bestimmen, können wir zwei verschiedene Wege einschlagen:

I. Wir bestimmen unmittelbar die Schnittpunkte der von den schatten werfenden Punkten ausgehenden Lichtstrahlen mit den Schatten

Fig. 72.

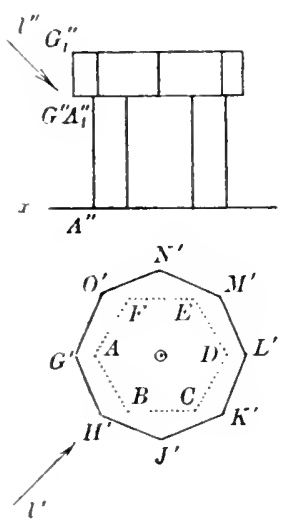

Fig. 73.
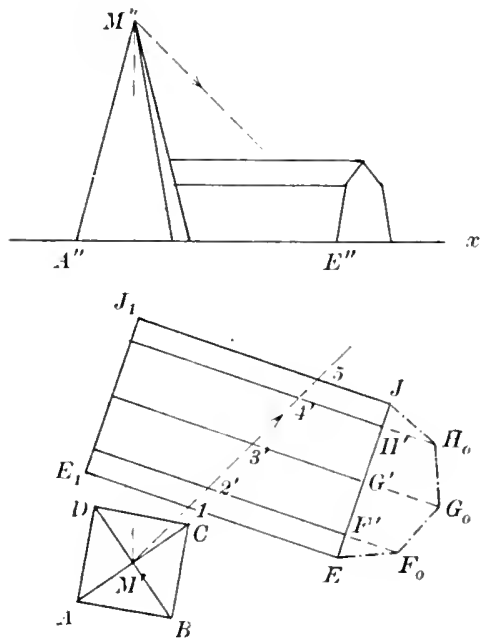

empfangenden Flächen unter Anwendung geeigneter Hilfsebenen, in der Regel mittels projizierender Ebenen durch die einzelnen Licht-trahlen (direktes Verfahren, vgl. Art.59).

a) Schatten einer achtseitigen prismatischen Platte auf pin sechsseitiges Prisma (Fig. 72). Die Eigenschattengrenze des Prismas besteht. wie man aus dem Grundriß erkennt, aus rlen Seitenkanten $C C_{1}$ und $F F_{1}$, die der Platte aus den Seitenkanten $K K_{1}$ und $O O_{1}$ und den halben Achtecken K.JH $H O$ und $K_{1} L_{1} M_{1} N_{1} O_{1}$. Um den Schatten der Kante $J I I$ auf die vertikale Fläche $B C C_{1} B_{1}$ zu konstruieren, bestimmt man den Schnittpunkt, $I_{*}$ des durch $I$ gehenden Lichtstrahles mit dieser Fläche, sowie den Schatten des Punktes von .$I I$, lessen Lichtstrahl die Kante $B B_{1}$ trifft, oder man verbindet $F_{*}$ mit dem Schatten des Punktes $I I$ anf die erweiterte Fläche. 
b) Schatten einer Pyramide auf ein Prisma (Fig. 73). Die Grundfläche $A B C l$ der Pyramide und die Prismakanten $E E_{1}$ und . $J J_{1}$ liegen in $\Pi_{1}$; die Grundflache des Prismas steht auf den Seitenkanten senkrecht und ist durch ihre Umlegung $I F_{0}\left(x_{0} I_{0} J\right.$ in $\Pi_{1}$ gegeben, es ist also Abstand $F^{\prime \prime} x=F^{\prime \prime} F_{0}$. Nan bestimme zunächst den Schatten $\boldsymbol{M}_{h}$ der Spitze $\boldsymbol{M}$ der Pyramisle auf $\Pi_{1}$. Dann schneiden die Grenzlinien $M_{h} B$ und $M_{h} D$ des von der Pyram de auf $\Pi_{1}$ geworfenen Schattens die Kante $E E_{1}$ in zwei Punkten $T$ und $U$; in ihnen beginnt der Schatten, den die Fläche $E F F_{1} E_{1}$ des Prismas von der Pyramide empfängt. Nun ermittle man die Schnittpunkte $M_{1}, M_{2} \ldots$ des durch $M$ gehenden Lichtstrahls mit den Seitenflächen des Prismas: Seine erste projizierende Ebene schneidet das Prisma in einem Fünfeck 12345 , und lie Gerade $M^{\prime \prime} M_{h}^{\prime \prime}$ trifft die Verlängerungen von $1^{\prime \prime} 2^{\prime \prime}$. $2^{\prime \prime} 3^{\prime \prime} \ldots$ bzw. in $\boldsymbol{M}_{1}^{\prime \prime}, \boldsymbol{M}_{2}^{\prime \prime} \ldots$ Dann gehen die Grenzen des auf die Fläche $E F F_{1} E_{1}$ fallenden Schattens von $T$ und $U$ nach $M_{1}$; sie schneiden die Kante $F F_{1}$ in zwei Punkten $V$ und $W$, die mit $M_{2}$ verbunden den Schatten auf die folgende Fläche liefern usw. Da die Schnittfigur 12345 von dem durch 4 gehenden Lichtstrahl - wie der Aufric zeigt - gestreift wird, so gehört die Kante $H H_{1}$ zur Eigenschattengrenze des Prismas; die Fläche $I / . J J_{1} I_{1}$ empfängt also keinen Schlagschatteu.

Der auf das Prisma fallende Schatten könnte auch mit Hilfe eines Seitenrisses konstruiert werden, wobei man die Ebene $\Pi_{3} \mathrm{zu}$ den Seitenflächen senkrecht zu stellen hätte. Benutzt man der Einfachheit wegen die Grundfläche als $\Pi_{3}$ und ermittelt die dritte Projektion des Lichtstrahls $\boldsymbol{M} M_{h}$, so schneidet $\boldsymbol{H}^{\prime \prime \prime} \boldsymbol{U}_{h}^{\prime \prime \prime}$ die Verlängerung von $E F_{0}$ im Punkte $M_{1}^{\prime \prime \prime}$ usw. - Dieses Verfahren empfiehlt sich besonders, wenn man von mehr als einem Punkte den Schatten auf das Prisma konstruieren muf.

Fig. 74.

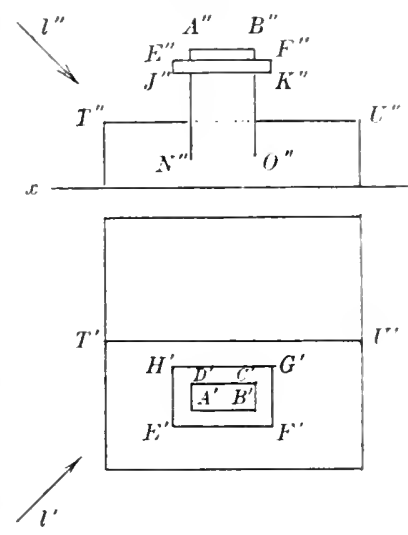

c) Schatten eines Schornsteins auf ein Dach (Fig. 7t). Der Schornstein besteht aus einem rechtwinkligen Parallelepiped mit der Deckfläche $A B C D$ und einem Gesims zwischen den horizontalen Rechtecken $E F G I I$ und $J K L M$. Sein Schnitt NOPQ mit der vorderen Dachfläche ergibt sich mittels des Seitenrisses auf eine $\Pi_{3}$, die auf dem First $T U$, im vorliegenden Falle also auf $x$ senkrecht steht und in die $\Pi_{2}$ umgelegt wird. - Die Eigenschattengrenze wird durch den Kantenzug $O B C D Q$ und das Sechseck $F G H M . J K$ gebildet. Der Schatten, den die Vertikale $O B$ auf die vordere Dachfläche wirft, beginnt in $O$. Sein Grundriß geht durch $B^{\prime}|| l^{\prime}$ und bestimmt seinen Schnitpunkt $I^{\prime}$ mit der Geraden $T U$; hieraus ergibt sich im Aufrif die Gerade $O^{\prime \prime} V^{\prime \prime}$. Zur Konstruktion des Schattens, den das Dach von der Eigenschattengrenze des Gesimses erhält, benutzt man den Seitenric (rgl. die Bemerkung am Schluf der vorhergehenden Aufgabe. Bei Licht in der 
Richtung der Würfeldiagonale bildet das ungelegte $l^{\prime \prime \prime}$ mit $x$ einen Winkel ron $45^{\circ}$ ). Die Kante $F G$ wirft Schatten auf beide Dachflachen; sein Schnittpunkt mit dem First wird aus dem Aufriß gefunden, der zu $l^{\prime \prime}$ parallel ist. Der Schatten der Kante $G H$ auf die hintere Dachfläche ist $\# G H$. Den parallelen Kanten $F G$ und $M J$ entsprechen parallele Schatten. - Die Kanten $M J$ und $J K$ werfen auch Schatten auf die belenchteten Schornsteinflächen. Endlich empfängt das Rechteck EFGH noch Schatten vom oberen Teile des Schornsteins. Die zugehörigen schattengrenzen ergeben sich unmittelbar aus Grund- und Aufrib.

70. II. Sind $a$ und $b$ zwei gerade (oder krumme) Linien im Raume, $a_{h}$ und $b_{h}$ ilire Schatten anf irgend eine Ebene, etwa auf die $\Pi_{1}$, und treffen sich $a_{h}$ und $b_{h}$ in einem Punkte $P_{h}$, so ist dieser der Schatten

Fig. 75.

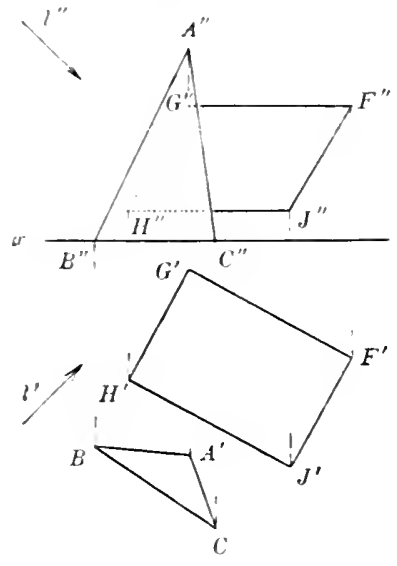
sowohl eines Punktes ron $a$ als auch eines Punktes ron $b$, d.h. der durch $I_{h}$ rückwärts gezogene Lichtstrahl schneidet beide Linien. Trifft er zuerst $a$ in $P$, hierauf $b$ in $Q$, so empfangt $a$ in $P$ schlagschatten rom Punkte $Q$ anf $h$.

Um luiernach in Fig. 75 den Schatten des Dreiecks $A B C$ auf das Rechteck FGH.J zu konstruieren, ermitteln wir zunächst den Schlagrschatten beider Figuren auf die $\Pi_{1}$. Dabei fällt der Schatten $A_{h}$ ron $A$ in das Parallelogramm $F_{h} G_{h} H_{h} J_{h}$, mithin wirft der Punkt $A$ seinen Schatten $A_{*}$ auf die Rechtecksfläche. Wir finden ihn mittels der Punkte $R_{h}$ und $S_{h}$, in denen $H_{h} \cdot J_{h}$ und $F_{h} G_{h}$ die Gerade $A_{h} B$ und deren Terlängerung schneiden: Die ersten Projektionen der durch diese Punkte gehenden Lichtstrahlen treffen $H^{\prime} J^{\prime}$ und $F^{\prime} G^{\prime}$ in $T^{\prime}$ und $S^{\prime}$; dann ist $R S$ der Schatten, den das Rechteck von der verlängerten Geraden $A B$ erhält, und der durch $A$ gehende Lichtstrahl schneidet $R S$ in $A_{*}$ (indirektes. Verfahren: Methode des Zurückprojizierens).

Ebenso konstruieren wir den Schatten, den das Vielflach A von leın Vielflach $B$ empfängt, aus dem Schatteu beider Körper auf die $\Pi_{1}$ : Da nur die beleuchteten Flächen von $A$ Schatten erhalten, und da die Grenzlinie dieses Schattens von der Eigenschattengrenze von B herrührt, so suchen wir in $\Pi_{1}$ die Schnittpunkte der Schattengrenze von B mit den Schatten der Kanten, die die beleuchteten Flïchen von A begrenzen, und projizieren die gefundenen Punkte in der Lichtrichtung auf die betreffenden Kanten von A.

\section{Der Kreis.}

71. Über ebene Kurren im allgemeinen. Liegen alle Punkte einer Kurve in einer Ebene, so heißt sie eben, anderenfalls wird sie als Raumkurve bezeichnet. 
Unter der 'Tangente der ebenen Kurve $k$ im Punkte $P$ versteht man bekanntlich die Grenzlage, die die Verbindungslinie von $P$ mit einem anderen Kurvenpunkte erreicht, wenn dieser sich dem Punkte $P$ auf $k$ unbegrenzt nähert; man sagt daher, die Tangente sei die Verbindungslinie zweier unendlich nahen Kurvenpunkte. - An gewissen singulären Stellen kann die 'Tangente mit der Kurve mehr als zwei unendlich nahe Punkte gemein haben. Ist ihre Anzahl $=3$ - oder überhaupt ungerade — so wird die Kurve von der 'Tangente im Berührungspunkte durchschnitten, und dann bezeichnen wir diesen als Inflexions- oder Wendepunkt. Liegen dagegen anf der Tangente vier unendlich nahe Kurvenpunkte - oder eine noch höhere gerade Zahl solcher Punkte - so bleibt die Tangente auf derselben Seite der Kurve; in diesem Ealle sprechen wir von einem Lndulations-oder Flachpunkt.

Das im Punkte $P$ zur Tangente errichtete Lot heißt die Normale der Kurve $k$ in $P$. - Durch $P$ und einen zweiten Punkt $Q$ ron $k$ läßt sich ein Kreis beschreiben, der $k$ in $P$ berührt; sein Mittelpunkt liegt auf der Normale von $P$. Rückt der Punkt $Q$ immer näher an $P$ heran, bis er schließlich mit $P$ zusammenfällt, so wird der betrachtete Kreis zum Krümmungskreis der Kurve $k$ in $P$. Wir können ihn auch definieren als den Kreis durch drei unendlich nahe Kurvenpunkte und seinen Mittelpunkt als den Schnittpunkt zweiel unendlich nahen Normalen. Der Krümmungskreis durchschneidet die Kurve in $P$, falls er nicht ausnahmsweise vier, oder überhaupt eine gerade Anzahl von Punkten mit der Kurve in $P$ gemein hat. - Alle Normalen von $k$ umhüllen eine zweite Kurve, nämlich den Ort der Krümmungsmittelpunkte der ersten; wir bezeichnen sie als die Evolute von $l$.

Wir entlehnen der analytischen Geometrie die folgenden Definitionen und Lehrsätze: Jede ebene Kurve ist darstellbar durch eine Gleichung

$$
\tilde{i}(\mathfrak{d}, \mathfrak{y})=0
$$

zwischen den rechtwinkligen Koordinaten $\mathfrak{x}, \mathfrak{y}$ ihrer sämtlichen Punkte. Ist $\dot{i}(\mathfrak{x}, \mathfrak{y})$ eine ganze rationale Eunktion von $\mathfrak{x}$ und $\mathfrak{y}$, so nennt man die Kurve algebraisch, und zwar von der $n^{\text {ten }}$ Ordnung, wenn die höchste Summe der Exponenten von $\mathfrak{x}$ und $\mathfrak{y}$ in einem Gliede $=n$ ist. Nichtalgebraische Kurven heiben transzendent. - Genügen komplexe Werte von $\mathfrak{x}$ und $\mathfrak{y}$ der Kurvengleichung, so sagt man, sie bestimmen einen imaginären Kurvenpunkt. Werden solche geometrisch nicht existierende Punkte mitgerechnet, so gilt der Satz: Eine ebene Kurve $n^{\text {ter }}$ Ordnung wird von jeder Geraden ihrer Ebene in $n$ Punkten geschnitten. Dabei sind aber alle Punkte, in denen wegen der besonderen Lage der Geraden zwei sonst getrennte Schnittpunkte in einen zusammenfallen - Berührungspunkte der Geraden, Doppelpunkte der Kurve - doppelt zu zählen. - In der Funktionentheorie wird umgekehrt bewiesen: Eine ebene Kurve, die von jeder Geraden ihrer Ebene in $n$ - reellen oder imaginären - Punkten geschnitten wird, ist eine algebraische Kurve $n^{\text {ter }}$ Ordnung. Es ist jedoch wohl zu beachten, dab bei der Bestimmung der Anzahl der Schnittpunkte nur das Entstehungsgesetz der Kurve und nicht der bloße Augenschein maßgebend ist. Aus der Gestalt allein läßt sich nicht 
einmal erkennen. ob eine gezeichnet vorliegende Kurve algebraisch oder transzendent ist.

72. Ist $k$ irgend eine Kurve in der Ebene $E$, $k^{\prime}$ ihre schiefe oder senkrechte Projektion auf die Bildebene $\Pi, r$ die Spur vou $E$, so sind $k$ und $l^{\prime}$ zwei perspektiv affine Figuren mit $e$ als Affinitaitsachse (Art. 49). Dabei entspricht der Tangente in einem Punkte P' von $k$ die 'Tangente im Punkte $P^{\prime}$ von $k^{\prime}$, dem beide Geraden sind die Verbindungslinien entsprechender Paare von unendlich nahen Punkten auf $k$ und $k^{\prime}$.

Den Schnittpunkten ron $k$ mit einer in $E$ liegenden Geraden $g$ sind der Reihe nach die Schnittpunkte von $k^{\prime}$ mit der Bildgeraden $g^{\prime}$ zugeordnet; daraus folgt: Die Parallelprojektion einer ebenen Kurve $n^{\text {ter }}$ Ordnung ist wieder ronder $n^{\text {ien }}$ Ordnung. - Derselbe Satz gilt offeubar auch für Zentralprojektion.

73. Ist die Kurve $k$ ein Kreis, so entsteht als Bildkurve $k^{\prime}$ eine im Endlichen geschlossene Kurve zweiter Ordnung; die Paral]elprojektion eines Kreises ist also eine Ellipse. Sie verwandelt sich in einen mit $k$ kongruenten Kreis, wenn $E \| \Pi$ ist, oder wenn die

Fig. is.

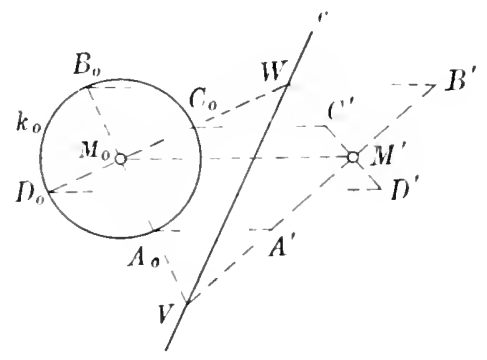

projizierenden Strahlen auf einer Halbierungsebene der von $E$ mit $\Pi$ gebildeten Wiukel senkrecht stehen ${ }^{1}$ ), und sie geht über in eine doppelt zählende Strecke, die dem Iurchmesser von $k$ gleich ist, wenu die projizierenden Strablen zu E parallel sind.

In Fig. 76 ist die Umlegung $k_{0}$ von $k$ um die Spurlinie $e$ in die Zeichenebene $\Pi$ gegeben, sowie rom Mittelpunkte $M$ die Projektion $M I^{\prime}$. Dann erhält man die Bildellipse $k^{\prime}$ als die perspektive affine Figur zu $k_{0}$ wie in Art. 49, indem man $z$ einer Reihe von Durchmessern $A_{0} B_{0}$, $C_{0} D_{0} \ldots$ von $k_{0}$ die entsprechenden Strecken $A^{\prime} B^{\prime}, C^{\prime} D^{\prime} \ldots$ konstruiert; dabei schneiden sich $A_{0} B_{0}$ und $A^{\prime} B^{\prime}$ auf der Affinitätsachse $e$, und die Geraden $A_{0} A^{\prime}$ and $B_{0} B^{\prime}$ sind $\| M_{0} M^{\prime}$. Hiernach ist $M^{\prime}$ aler Mittelpunkt von $A^{\prime} B^{\prime}, C^{\prime} D^{\prime} \ldots$, d. h.: Die Projektion des Kreismittelpunktes ist der Mittelpunkt der Bildellipse; jedem Kroisdurchmesser entspricht als Bild ein Ellipsendurchmesser.

Stelien die Kreisdurchmesser $A_{0} B_{0}$ und $C_{0} D_{0}$ aufeinander senkrecht, so ist jeder von ihnen parallel zu den 'Tangenten in den Endpunkten des anderen, und jeder halbiert die Sehnen, die zum anderen parallel sind; dasselbe grilt also auch von den Durchmessern $A^{\prime} B^{\prime}$ und $C^{\prime} D^{\prime}$ von $k^{\prime}$. Solche Durchmesser einer Ellipse nennt man einander konjugiert, mithin ergiht sich der Satz: Zwei a ufeinandersenkrechten Kreisdurchmessern entsprechen zwei konjugierte Ellipsendurchmesser.

1) Vql. Anmerkung zu Art. $3 x$ 
Die konjugierten Durchmesser $A^{\prime} B^{\prime}$ und $C^{\prime} D^{\prime}$ bilden nur dann einen rechten Winkel, wenn die Punkte $V$ und $W$, in denen sie sich mit $A_{0} B_{0}$ und $C_{0}^{\prime} D_{0}$ auf der Geraden $e$ schneiden, mit $M_{0}$ und $M^{\prime}$ auf einem hreise liegen, der seinen Mittelpunkt auf $e$ hat. In diesem Falle heilsen $A^{\prime} B^{\prime}$ und $C^{\prime \prime} D^{\prime}$ die Achsen der Ellipse $k^{\prime}$. Wir gelangen somit zur Lösung der folgenden Grundaufgabe: Vom Kreise $k$ ist die Spur $e$ seiner Ebene, seine Umlegung $k_{0}$ in die Zeichenebene $\Pi$ und von seinem Mittelpunkte $M$ das Bild $I^{\prime}$ gegeben; die Achsen der Bildellipse $k^{\prime} z u$ konstruieren. IIan bestimue den Schnittpunkt $O$ von $e$ mit der Mittelsenkrechten von $M_{0} M^{\prime}$, beschreibe um $O$ mit dem Radius $O \boldsymbol{M}_{0}$ einen Kreis, der $e$ in $V$ und $W$ schneidet, und ziehe in $k_{0}$ nach $V$ und $W$ die Durchmesser $A_{0} B_{0}$ und $C_{0} D_{0}$. Die durch ihre Endpunkte gehenden Parallelen zu $\boldsymbol{M}_{0} \boldsymbol{M}^{\prime}$ bestimmen auf $M^{\prime} V$ und $M^{\prime} W$ die Achsenendpunkte $A^{\prime}, B^{\prime}$ und $C^{\prime}, D^{\prime}$.

Auf dem Hilfskreise um $O$ befindet sich auch der Punkt $\boldsymbol{M}_{*}$, der in bezug auf $e$ zu $M_{0}$ symmetrisch liegt. Dann ist $V$ der Mittelpunkt des Bogens $\boldsymbol{I}_{0} \boldsymbol{M}_{*}$; ist also $O$ unerreichbar, so erhält man $\boldsymbol{M}^{\prime} V$ als Halbierungslinie des Winkels $M_{0} \mu^{\prime} \boldsymbol{H}_{*}$.

Bei senkrechter Projektion ist bekamntlich $M_{0} M^{\prime} \perp e$ und Abstand $\boldsymbol{M}^{\prime} e<$ Abstand $\boldsymbol{M}_{0} e$. Dann werden alle Durchmesser ron lio durch Projektion verkürzt, mit Ausnahme des zu $e$ parallelen Durchmessers $A_{0} B_{0}$; diesem entspricht also der größte Durchmesser der Ellipse, d. h. ihre grobe Achse $A^{\prime} B^{\prime}$ \# $A_{0} B_{0}$.

74. Sind von einer Ellipse, die wir jetzt lieber mit $k_{i}$ statt mit $k^{\prime}$ bezeichnen wollen, zwei konjugierte Durchmesser $A B$ und $C D$ gegeben. und beschreiben wir über einem von ihnen, z. B. über $A B$, um den Mittelpunkt $M$ ron $k$ den Kreis $k_{0}$, so können wir $k$ als die perspektiv affine Kurve zu $k_{0}$ betrachten mit $A B$ als Affinitätsachse (Fig. 77 ). Dabei entspricht dem Ellipsendurchmesser $C D$ der auf $A B$ senkrechte Kreisdurchmesser $C_{0} D_{0}$; die Affinitätsstrahlen sind also $\| C_{1} C$. Hieraus ergibt sich die folgende Konstruktion der Ellipse $k$ aus den konjugierten Durchmessern $A B$

Fig. 77.

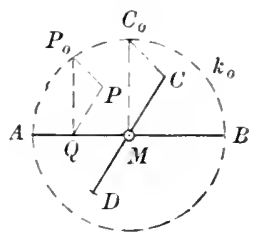
und $C D$ : Man fälle von einem beliebigen Punkte $P_{0}$ von $l_{0}$ auf $A B$ das Lot $P_{0} Q$ und ziehe $Q P \| M C$ und $P_{0} P \| C_{0} C$; dann ist $P$ ein Punkt von $k$, denn der zu $l_{i}$ gebörenden Geraden $P_{0} Q$ entspricht in der affinen Figur die Gerade $I^{\prime}($.

Die Ellipsentangente in $P$ geht durch den Schnittpunkt der Kreistangente in $P_{0}$ mit der Affinitätsachse $A B$.

Ist von einer Ellipse ein Durchmesser $A B$, die Richtung des konjugierten Durchmessers und ein Punkt $P$ gegeben, so findet man die Länge des konjugierten Halbmessers $\boldsymbol{M} C$ mit Hilfe des Dreiecks $P_{0} Q P$ und des ihm ähnlichen und parallel liegenden Dreiecks $C_{0} M C$.

75. Die affine Beziehung zwischen der Ellipse $l_{i}$ und dem Kreise $k_{0}$ liefert ein bequemes Mittel, um eine Reilıe von Konstruktionsaufgaben über die Ellipse durch Zurückführung auf dieselbe Aufgabe am Kreise zu lösen. Wird z. B. verlangt, 
an die durch zwei konjugierte Durchmesser $A B$ und $C D$ gegebene, aber nicht gezeichnete Ellipse $k$ aus einem beliebigen Punkte $R$ der Ebene Tangenten zu zielien, so konstruiere man zu $R$ den in der Kreisfigur entsprechenden Punkt $R_{0}$ ( $R S \| C M$ bis $\left.A B, S R_{0} \perp A B, l i R_{0} \| C C_{0}\right)$, lege aus $R_{0}$ an den Kreis $k_{0}$ Tangenten und verbinde $R$ mit den Punkten, in denen jene die Affinitätsachse $A B$ schneiden.

Un an dieselbe Ellipse Tangenten von gegebener Richtung zu legen, ziehe man in dieser Richtung die Gerade $C U$ bis $A B$ und an den Kreis $k_{0}$ Tangenten $\| C_{0} U$.

Ebenso findet man die Schnittpunkte einer Gieraden $g$ mit der nicht gezeichneten Ellipse mit Hilfe der entsprechenden Sekante $g_{0}$ des Kreises $k_{0}$ : Zieht man durch den Schnittpunkt $T$ von $g$ mit $M C$ die Gerade $T T_{0} \| C C_{0}$ bis $M C_{0}$, so geht $g_{0}$ durch $T_{0}$ und durch den Scbnittpunkt vou $g$ mit $A B$ usw.

76. Konstruktion einer Ellipse ans ihren Achsen $A B=2 a$ und $C D=2 b$ (Fig. 78). Die Ellipse $k$ ist wieder perspektiv affin $z u$ dem Kreise, der $A B$ zum Durchmesser hat; wir bezeichnen ihn gegenwärtig mit $k_{1}$. Dem Punkte $C$ ron $k$ entspricht der Schnittpunkt $C_{1}$

Fig. 78.

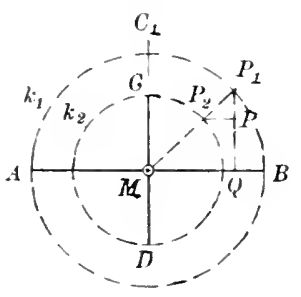
von $k_{1}$ mit der verlängerten Halbachse $\| C$; einem beliebigen Punkte $P_{1}$ ron $k_{1}$ ist also nach Art. 74 der Punkt $P$ von $k$ zugeordnet, der die auf $A B$ senkrechte Strecke $P_{1} Q$ in demselben Verhältnis teilt, wie $C$ die Strecke $C_{1} M$. Beschreiben wir daher um $\boldsymbol{M}$ mit dem Radius $M C$ den Kreis $k_{2}$, der $M P_{1}$ in $P_{2}$ schneidet, so erhalten wir $P$ als Schnittpunkt von $P_{1} Q$ mit der Parallelen durch $P_{2}$ zu $A B$.

Verlängern wir $M P_{1}$ um $P_{1} P_{3}=b$, so ist $P P_{3}$ die Normale der Ellipse $k$ in $P$. Denn die Tangente von $k$ in $P$ geht durch den Schnittpmint $V$ von $A B$ mit der 'Tangente ron $k_{2}$ in $P_{1}$; dann folgt aus der Ähnlichkeit der Dreiecke $P_{1}^{\prime} P P_{2}$ und $V P_{1} M$, daß auch die Dreiecke $P I_{2} P_{3}$ und $P P_{1} V$ einander ähnlich sind, mithin ist $\angle P_{3}^{\prime} Y P_{2}=\angle V P P_{1}$, also $\angle V P P_{3}=90^{\circ}$.

Sind $R$ und $S$ die Schnittpunkte von $A B$ nnd $C D$ mit der Parallele durch $P$ zu $M P_{1}$, so ist $P R=b, P S=a$. Gleitet daher die Strecke $R S=a-b$ mit den Punkten $R$ und $S$ anf den Achsen $A B$ und $C D$, so beschreibt der anf ihrer Verlängernng liegende Punkt $P^{\prime}$ die Ellipse. - Machen wir ferner auf $A B$ die Strecke $Q T=R Q$ und ziehen ' $T P$ bis $U$ auf $C D$, so wird $P U=a, P^{\prime} T=b$; die Ellipse entsteht also auch als Bahnkurve von $P$, wenn die Endpnnkte der Strecke $T U=a+b$ sich bzw. auf $A B$ und $C D$ bewegen (Papierstreifenkonstruktionen der Ellipse).

Hieraus folgt beiläufig: Kennen wir von einer Ellipse die eine Achse $A B=2 a$ und einen beliebigen Punkt $P$, so finden wir die Länge $b$ der anderen Halbachse, indem wir um $l$ mit $a$ einen Kreisbogen beschreiben. Trifft dieser die nicht gegebene Achse in $S$ und schneiden wich $P S$ und $A B$ in $R$, so ist $P R=b$. 
77. Um die Ellipse $k$ möglichst genau zu zeichnen, ermitteln wir noch ihre Scheitelkrümmungskreise. Sei $P_{*}$ der Punkt von $k$, der zu $P$ in bezug auf $A B$ symmetrisch liegt, $\varkappa$ der Kreis durch $P$ und $P_{*}$, der $k$ in $B$ berührt, und $W$ sein zweiter Schnittpunkt mit der Achse $A B$ : dann ergibt siclı aus dem rechtwinkligen Dreieck $B P W$

$$
P Q^{2}=B Q \cdot Q W
$$

und aus dem Dreieck $B P_{1} A$

$$
P_{1} Q^{2}=B Q \cdot Q A
$$

mithin ist

$$
\frac{P Q^{2}}{P_{1} Q^{2}}=\frac{Q W}{Q A}
$$

Nun verhält sich

$$
\frac{P Q}{P_{1} Q}=\frac{b}{a}
$$

folglich ist

$$
\frac{Q W}{Q A}=\frac{b^{2}}{a^{2}}
$$

Lassen wir $Q$ immer näher an $B$ heranrücken, bis schlieblich $Q, P$ und $P_{*}$ mit $B$ zusammenfallen, so verwandelt sich $\boldsymbol{x}$ in den Krümmungrskreis der Ellipse in ihrem Scheitel $B$; dieser ausgezeichnete Krümmungskreis hat also mit der Ellipse in $B$ nicht nur drei, sondern vier unendlich nahe Punkte gemein (vgl. Art. 71). Bezeichnen wir seinen Radius mit $\varrho_{1}$, so wird beim Grenzübergang $Q W=2 \varrho_{1}, Q A=2 a$, und dann folgt aus der letzten Gleichung

$$
\varrho_{1}=\frac{b^{2}}{a} .
$$

Denselben Wert hat der Krümmungsradius in $A$, und für die Krümmungsradien der Scheitel $C$ und $D$ ergibt sich durch Tertauschung von $a$ und $b$ der Ausdruck

$$
\varrho_{2}=\frac{a^{2}}{b} \text {. }
$$

Zeichnen wir daher in Fig. 78 das Rechteck $B M C E$, so trifft das Lot von $E$ auf $B C$ die Achsen $A B$ und $C D$ bzw. in den Krümmungsmittelpunkten der Scheitel $B$ und $C$.

78. Konstruktion der Achsen einer Ellipse aus einem Paar konjugierter Durchmesser. Wir bezeichnen wieder mit $\boldsymbol{M}$ den Mittelpunkt, mit $A B$ und $C D$ die Achsen einer Ellipse $k$, mit $k_{1}$ und $k_{2}$ die Kreise über den Durchmessern $A B$ und $C D$ (Fig. 79). In $k_{1}$ ziehen wir irgend zwei aufeinander senkrechte Radien $M E_{1}$ und $M G_{1}$, schneiden sie mit $k_{2}$ in $E_{2}$ und $G_{2}$ und bestimmen nach Art. 76 zu $E_{1}$ und $G_{1}$ die entsprechenden Ellipsempunkte $E$ und $G$ mittels $E_{1} E \perp A B$, $E_{2} E \| A B$ usw. Dann sind $M E$ und $M G$ zwei konjugierte Halbmesser von $k$, und es ist $\Delta E_{1} E E_{2} \simeq \Delta G_{2} G G_{1}$. Drehen wir $\Delta M G_{1} G$ um $M$, bis $G_{1}$ mit $E_{1}$, also $G_{2}^{\prime}$ mit $E_{2}$ zusammenfällt, so gelangt $M G$ nach $M J \perp M G$. In dem Rechteck $E_{1} E E_{2} J$ halbieren sich die Diagonalen 
in $O$; sind also $S$ und $T$ ' die Schnittpunkte von $F . J$ mit $A B$ und $C D$, so ist $O S=O T=O M$ und $M E_{1}=J S=M A$, sowie $M E_{2}$ $=J T=M C$.

Kennen wir daher umgekehrt von der Ellipse $k$ die konjugierten Dnrchmesser $E F$ und $(i M$, so finden wir die Achsen in folgender Weise: Wir ziehen $M \cdot I \equiv M G$ und beschreiben um den Mittelpunkt $O$ von $E \cdot J$

Fig. 79.
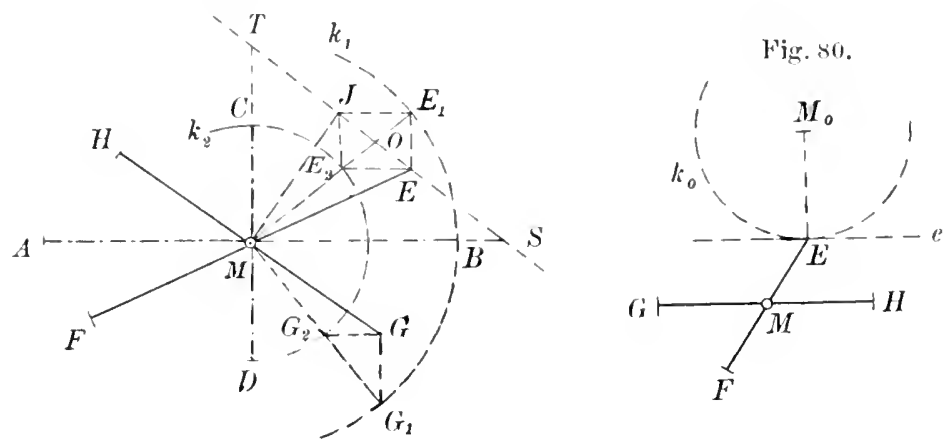

mit $O M$ eimen Kreisbogen, der $E J$ in $S$ und $T$ schneidet. Dann gehen die Achsen $A B$ und $C D$ bzw. durch $S$ und $T$, und zwar ist $M A=J S$ und $M C=J T$.

, Eine zweite Lösung derselben Aufgabe ergibt sich aus Fig. 80, in der $E F$ und $(i H$ wie vorher ein Paar konjugierte Durchmesser der Ellipse $k$ bezeichnen. Ziehen wir in $E$ die Tangente $e \| G M$ und senkrecht dazu die Gerade $E M_{0}=M\left(i\right.$, und beschreiben wir um $M_{0}$ den Kreis $k_{0}$ durch $E$, so ist $k$ perspektiv affin $z u k_{0}$ mit $e$ als Affinitätsachse und $M_{0}$ als Richtmng der Affinitätsstrahlen. Daun erbalten

Fig. 81 . wir die Achsen von $k$ wie bei der in Art. 73 be-
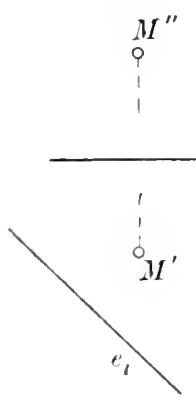
wird. Ziehen wir also durch $\Pi^{\prime}$ die (ierade $A^{\prime} B^{\prime} \| c_{1}$ und machen $M^{\prime} A^{\prime}=M^{\prime} B^{\prime}=r$, so ist $A^{\prime} B^{\prime}$ die grobe Achse von $k^{\prime}$. Die zugehörige kleine Achse ist der Grundrib des auf $A B$ senkrechten Kreisdurchmessers $(I)$. Dieser geht durh den Fubunkt of des Lotes von $M^{\prime}$ auf $e_{1}$. Durch Umlegung des rechtwinkligren Dreiecks $M M^{\prime} . J$ in $\Pi_{1}$ gelangt $M$ nach $M_{0}$ auf $M^{\prime} \Lambda^{\prime}$; dabei ist $M^{\prime} M_{0}=$ Abstand $M^{\prime \prime} x$. 
Machen wir auf $J \boldsymbol{M}_{0}$ die Strecke $\boldsymbol{M}_{0} C_{0}=r$, so ist $C^{\prime}$ der Fubpunkt des Lotes von $C_{0}$ auf $J \boldsymbol{M}^{\prime}$.

Als Aufrib erhalten wir eine Ellipse $k^{\prime \prime}$ mit dem Mittelpunkte $\boldsymbol{M}^{\prime \prime}$. Ihre große Achse ist die Projektion des zu $\Pi_{2}$ parallelen Kreisdurchmessers $E F$ und $=2 r$. Ziehen wir $M L^{\prime} N \| x$ bis $e_{2}$ und $N N^{\prime \prime} \perp x$. so liegen $E^{\prime \prime}$ und $F^{\prime \prime}$ auf $M^{\prime \prime} N^{\prime \prime}$. Von der Ellipse $k^{\prime \prime}$ kennen wir ferner den Punkt $A^{\prime \prime}$ auf der Parallelen durch $M^{\prime \prime}$ zu $x$ : wir finden daher die kleine Halbachse nach der Schlußbemerkung in Art. 76 .

Der Schlagschatten von $k$ auf $\Pi_{1}$ ist eine Ellipse $k_{h}$, die den Schatten $M_{h}$ von $M$ zum Mittelpunkt hat. Bilden wir von $k$ die Imlegung in $\Pi_{1}$, so finden wir die Achsen von $k_{h}$ nach der Grundaufgabe in Art. 73 .

80. Anwendung. Konstruktion eines sogenannten Winkelriementriebs. Soll über zwei kreisförmige Scheiben mit windschiefen Achsen ein geschlossener Riemen gelegt werden, der die Drehung der eineu Scheibe um ihre Achse auf die andere überträgt, so muß man bekanntlich dafür sorgen, daß bei jeder Scheibe die Mittellinie Fig. 82.

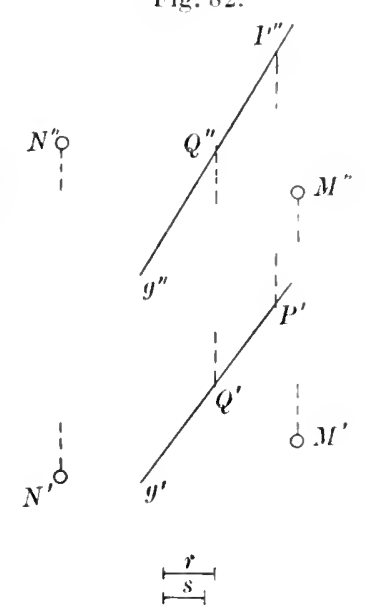
des anliegenden Riemenstücks in die Mittelebeue der Scheibe zu liegen kommt, und dies erreicht man durch Einführung zweier Leitrollen. Fig. 82 behandelt die Aufgabe in ihrer

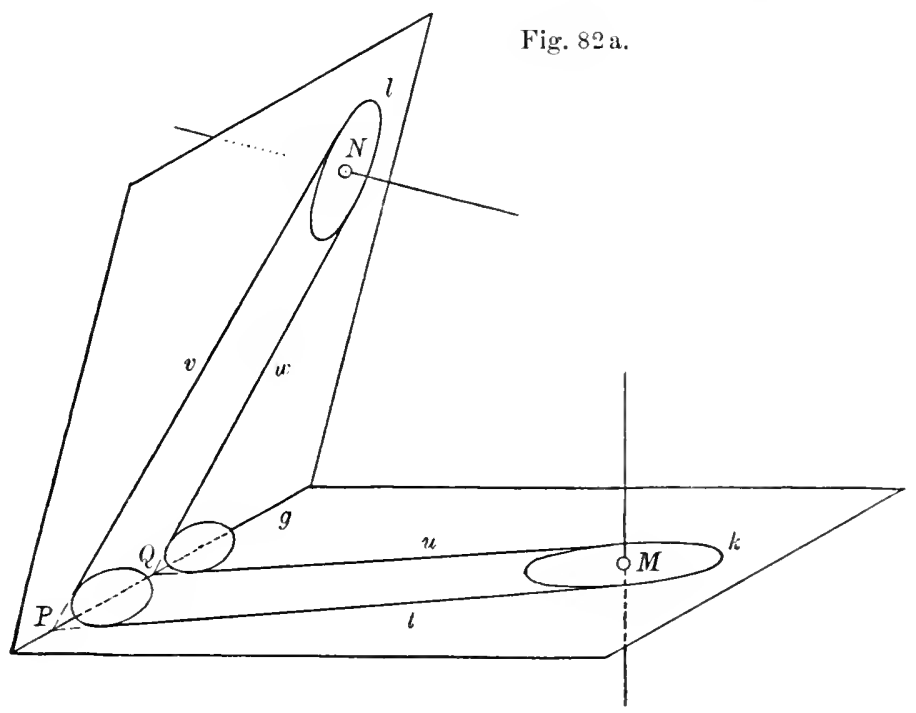

allgemeinsten Form (vgl. auch die Skizze 82a). Als Mittelschnitte der beiden Scheiben sind die Kreise $k$ und $l$ gegeben durch ihre Mittel- 
punkte $M$ und $N$, die Radien $r$ und $s$ und die Schnittlinie $g$ ihrer Ebenen. Man zeichue zuächst von jedem der beiden Kreise seinen Grund- und Anfrib, am einfachsten aus den Projektionen des zu $\Pi_{1}$ und des zu $\Pi_{2}$ parallelen Durchmessers; dadurch erlält man nämlich von jeder Bildellipse die große Achse und einen zweiten Durchmesser, wodurch die kleine Achse bestimut ist. Darauf riche man aus zwei passend gewählten Punkten $P$ und $Q$ von $g$ die 'T'angenten $t$ und $u$ an $k$. sowie $r$ und $w$ an 7 ; diese Geraden sind die Mittellinien der die Scheiben berührenden Riemenstücke. Konstruiert man endlich mit geeigneten Radien zwei Kreise, von denen der eine $t$ und $v$, der andere $u$ und $w$ berührt, so sind die Mittelschnitte der beiden Leitrollen ge-

Fig. 83.
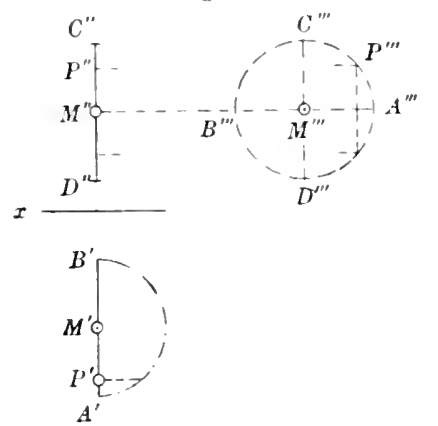

funden. Die Ausführung dieser Konstruktion erfordert selbstrerständlich die Umlegung jeder der Ebenen $t v$ und $u w$ in eine Projektionsebene, oder die Drehung jecler einzelnen Ebene um eine Hauptlinie, bis sie zur betreffenden Projektionsebene parallel wird.

81. Darstellung eines Kreises $k$, dessen Ebene auf der Projektionsachse $x$ senkrecht steht; gegeben ist der IIittelpunkt $M=M I^{\prime}$, $\boldsymbol{M}^{\prime \prime}$ und der Radius $r$ (Fig. 83). Bezeichnen wir mit $A B$ und $C D$ die Durchmesser ron $k$, die bzw. zu $\Pi_{1}$ und $\Pi_{2}$ parallel sind, so ist $k^{\prime}=A^{\prime} B^{\prime}, k^{\prime \prime}=C^{\prime \prime} D^{\prime \prime}$. Um zum Punkte $P$ von $k$, dessen Grandrib $P^{\prime}$ bekannt ist, den Aufrif $P^{\prime \prime}$ zu bestimmen, benutzen wir den Seitenrif $k^{\prime \prime \prime}$, umgelegt in $\Pi_{2}$; dann ist Abstand $P^{\prime \prime \prime}, C^{\prime \prime \prime} D^{\prime \prime \prime}=P^{\prime} M^{\prime}$. Selbstrerständlich genügt auch eine Drelıng des vorderen Halbkreises um $C I$ ), bis er $\| \Pi_{2}$ wird. Oder wir drehen $l_{i}$ um $A B$ in die horizontale Lage $k_{0}$; dann ist $P^{\prime} P_{0}^{\prime} \perp A^{\prime} B^{\prime}$ und $M^{\prime \prime} P^{\prime \prime}=P^{\prime} I_{0}^{\prime}$.

\section{Die Kugel.}

82. Über krumme Flächen im allgemeinen. Unter einer 'Tangente einer Fläche im Punkte $P$ versteht man seine Verbindungslinie mit einem unendlich nahen Flächenpunkte. Sämtliche Tangenten Jer Fläche in $P$ bilden eine Ebene, die Berührungsebene der Fläche in diesem Punkte. Eine Ausnahme machen gewisse "singuläre Flächenpunkte, z. B. bei einer Fläche, die durch Drehung einer Kurve um eine sie nicht rechtwinklig schneidende Achse entsteht, der Schnittpunkt mit der Achse.

Der geometrische Ort aller Flächenpunkte, deren projizierende Strahlen die Flache berühren, heilt der wahre Umriß der Fläche, seine Projektion der scheinbare Umrib. - Liegt a u einer Fläche e ine Kurve $k$, die den wahren Lmriß $u$ im Punkte $T$ schneidet, so berührt die Projektion $l^{\prime}$ der Kurve den scheinbaren Umri@ $u^{\prime}$ in $T^{\prime}$; denn die langenten von $l$ und $u$ in $T$ haben dieselbe 
Projektion, weil die Berührungsebene rer Fläche in $T$ außer den gønannten 'Tangenten auch den projizierenden Strahl $T T^{\prime}$ euthält.

83. Darstellung einer Kugel in Grund- nnd Aufrib; gegeben der Mittelpunkt $\boldsymbol{M}\left(\boldsymbol{M}^{\prime}, \boldsymbol{M}^{\prime \prime}\right)$ und rer Radius $r$ (Fig. 84). Der. erste und der zweite wahre Umrif der Kugel sind die Hauptkreice $u \| \Pi_{1}$ und $v \| \Pi_{.2}$. Der erste scheinbare Umrif ist also der Kreis $\iota^{\prime}$ um $H^{\prime}$ mit dem Radius $r$; seine zweite Projektion fällt zusammen mit lem zu $x$ parallelen Durclimesser des zweiten scheinbaren Umrisses $v^{\prime \prime}$. Alle Punkte der oberhalb $u$ liegenden Halbkugel sind im Grundriß sichtbar; ihre zweiten Projektionen erfüllen die obere Halbkreistläche $v^{\prime \prime}$.

Un von dem Punkte $Y$ der Kugelfläche, der durch seinen Grundrib $P^{\prime}$ gegeben ist, den Aufrif zu konstruieren, benutzen wir den durch $P$ gehenden horizontalen Kugelkreis $p$. Sein Grundriß ist der Kreis $\varphi^{\prime}$ um $M^{\prime}$ durch den Punkt $P^{\prime}$. Jedem Schnittpunkte von $p^{\prime}$ mit $v^{\prime}$ entsprechen in

Fig. 84.

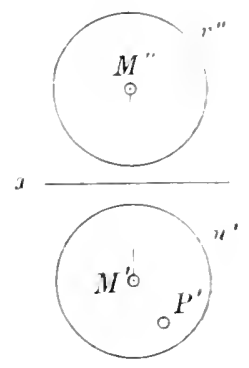
Aufrib zwei Punkte von $v^{\prime \prime}$ : die zugehörigen zu $x$ parallelen Sehnen sind die zweiten Projektionen der beiden Kugelkreise, die $p^{\prime}$ zum Grundrí haben. - Statt des Kreises $p$ können wir auch den Kugelkreis verwenden, der durch $P \| \Pi_{2}$ gelegt wird.

84. Aufgabe. Den Schnitt der Ebene $\mathrm{E}\left(e_{1} e_{2}\right)$ mit einer Kugelvom Iittelpunkt $M$ zu konstruieren (Fig. 85 ). Die Schnittkurve ist ein Kreis $k$, sein Mittelpunkt der Fubpunkt $O$ des von $M$ auf $E$ gefallten Lotes. Wie bei der Konstruktion des ebenen Schnitts eines Vielflachs bedienen wir uns einer dritten Projektionsebene $\Pi_{3} \perp e_{1}$, die $\Pi_{1}$ in einer Geraden $y \perp e_{1}$ schneidet, und bestimmen in der Umlegung in $\Pi_{1}$ die dritte Spur $e_{3}$ (Art. 46), sowie den Punkt $I^{\prime \prime \prime}$ und den dritten scheinbaren Umriß $w^{\prime \prime}$ der Kugel, also die dritte Projektion des zu $\Pi_{3}$ parallelen Hauptkreises $u$. Die auf $e_{3}$ liegende Sehue $C^{\prime \prime \prime} D^{\prime \prime \prime}$ von $w^{\prime \prime \prime}$ ist die dritte Projektion und zugleich die wahre Größe des auf $e_{1}$ senkrechten Durchmessers CD von $k$, sowie die dritte Projektion des Schnittkreises selbst. Um die Fig. 85 .

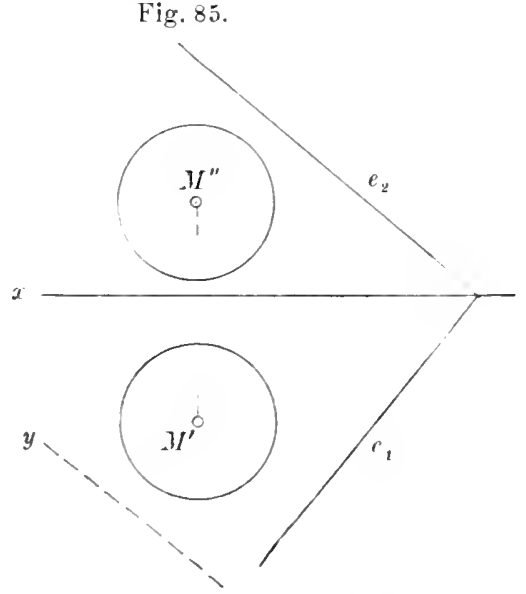
Projektionen des Mittelpunktes $O$ zu erhalten, ziehen wir $M^{\prime \prime \prime} O^{\prime \prime \prime} \perp e_{3}$, $M^{\prime} O^{\prime} \perp e_{1}, O^{\prime \prime \prime} O^{\prime} \perp y, M^{\prime \prime} O^{\prime \prime} \perp e_{2}$. Der Grundrib von $k$ ist eine Ellipse $k^{\prime}$ mit dem Mittelpunkt $O^{\prime}$, der kleinen Achse $C^{\prime} D^{\prime} \perp e_{1}$ und der großen Achse $A^{\prime} B^{\prime}=C^{\prime \prime \prime} D^{\prime \prime \prime}$. Die große Achse der Ellipse $l^{\prime \prime}$ ist $\| e_{2}$ und ebenfalls $=C^{\prime \prime \prime} I^{\prime \prime \prime}$; die zugehörige kleine Achse ergibt sich wie in Art. 79 mittels des Punktes $A^{\prime \prime}\left(O^{\prime \prime} A^{\prime \prime} \| x\right)$. 
Der erste Umrilj $u$ schneidet $k$ in zwei Punkten $T$ und $U$, den Endpunkten des im Grundrib sichtbaren Teils von $k$. Im Seitenrib

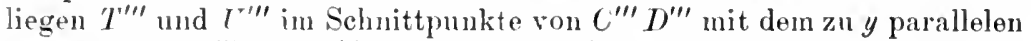
Durchmesser $u^{\prime \prime \prime}$ von $w^{\prime \prime \prime}$. Die Ellipse $k^{\prime}$ berührt den Kreis $u^{\prime}$ in $T^{\prime \prime}$ und $U^{\prime}$ (Art.82). - Wir bestimmen ferner die Berührnngspunkte $V^{\prime \prime}$ und $\|^{\prime \prime}$ ven $k^{\prime \prime}$ mit dem zweiten scheinbaren Umrib $v^{\prime \prime}$ : Die Ebene von $v$ schneidet $\mathrm{E}$ in einer zweiten Hauptlinie, und diese trifft $v$ in $\Gamma$ und $T$.

Die Lësung der Aufgabe gestaltet sich noch etwas einfacher, wenn wir die Seitemribebene durch den Kugelmittelpunkt legen und sie um ihre Schnittlinie mit der Ebene von u drehen, bis sie mit dieser zusammenfiallt.

85. Aufgabe. Den S'chnitt des Dreiecks A $B C^{\prime}$ mit einer kugel vom Mittelpunkt $M$ zu koustruieren (Fig. 86). Wir bestimmen eine hinreichende Anzahl von Punkten des Schnittkreises $k$, indem wir durch die Kugel und das Dreieck eine Reihe horizontaler

Fin. 86 .
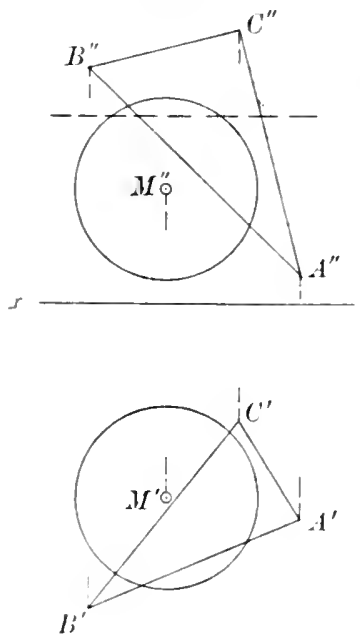

Hilfsebenen legen. Eine solche Ebene schneidet die Kugel in einem Kreise $p$, das Dreieck in einer Geraden ST, und dann geht $k$ durch die Schnittpunkte von $p$ mit $S T$, die zuerst im Grundri gefunden werden. Dabei kommen aber nur solche Schnittpunkte in Betracht, die innerhalb der begrenzten Dreiecksfläche liegen. So ermitteln wir insbesondere die Punkte im ersten Umriß ; auberdem konstruieren wir, wie bei der vorhergehenden Aufgabe, die Punkte im zweiten Umriß $v$.

Um die Schnittpunkte $D$ und $E$ der Kugel mit der Geraden $A B$ zu bestimmen, schneiden wir die Kugel mit der ersten projizierenden Ebene von $A B$ in einem Kreise $i$, dessen Grundrib mit der auf $A^{\prime} B^{\prime}$ liegenden Selıne $F^{\prime} G^{\prime}$ von $u^{\prime}$ zusammenfällt. Sein Mittelpunkt $O$ ist der Fußpunkt des Lotes von $M I$ auf diese Ebene, also von $\Pi_{1}$ ebenso weit entfernt wie $\boldsymbol{M}$. Die gesuchten

Punkte $D$ und $E$ sind die Schnittpunkte von $A B$ mit $i$. Um die Konstruktion der Ellipse $i^{\prime \prime}$ zu vermeiden, legen wir die Ebene $A B B^{\prime} A^{\prime}$ in die $\Pi_{1}$ um. Dann gelangen $A B, O$ und $i$ bzw. nach $A_{0} B_{0}, O_{0}$ und $i_{0}$ : dabei ist $A^{\prime} A_{0} \perp A^{\prime} B^{\prime}$ und $=$ Abstand $A^{\prime \prime} x, M^{\prime} O_{0} \perp A^{\prime} B^{\prime}, O^{\prime} O_{0}$ $=$ Abstand $M^{\prime \prime} x$ und der Radius von $i_{0}=O^{\prime} F^{\prime \prime}$. Die Gerade $A_{0} B_{0}$ trifft $i_{0}$ in $D_{0}$ und $E_{0}$ usw.

86. Die Konstruktion des ebenen Schnittes einer Kugel vereinfacht sich beträchtlich, wem die schneidende Ebene anf einer Projektionsebene senkrecht steht:

a) Darstellung einer Hängekuppel. In $\Pi_{1}$ ist das Quadrat A $B C D$ gegeben. Wie durch seine Eckpunkte gelegte Halbkugel ist mit vertikalen Ebenen: die durch dio Seiten gehen, zn schneiden (Fig. 87). 
Die zweiten Projektionen der so entstehenden Halbkreise (II auerbögen) sind Halbellipsen, die aus ihren Achsen sofort konstruiert werden können. Wir bestimmen auberdem die Schnittpunkte $V$ unt IV der Halbkreise über $B C$ und $A D$ mit dem zweiten Kugelumri $v$ : nach Wegnahme der abgeschnittenen Kugelteile reicht dann der zweite scheinbare Umriß $v^{\prime \prime}$ nur von $V^{\prime \prime}$ bis $W^{\prime \prime}$.

b) Darstellung einer böhmischen Kappe. In $\Pi_{1}$ ist das Rechteck $A B C D$ und senkrecht über dem Schnittpunkte $O$ der Diagonalen der Gewölbescheitel $S$ gegeben; dabei ist die Stichböhe OS kleiner als $O A$. Die durch die Punkte $A, B, C, D, S$ gelegte Kugelkappe ist mit vier vertikalen Ebenen, die durch die Seiten des Rechtecks gehen, zu begrenzen (Fig. 88). (Tm zunächst den Mittelpunkt $M$

Fig. 87.

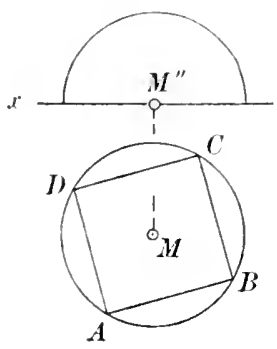

Fig. 88.

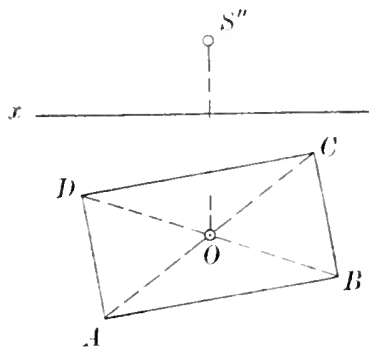

der Kugel zu finden, drehen wir die vertikale Ebene $A S C^{\prime}$ um $O S$, bis sie $\| \Pi_{2}$ wird. Kommt hierdurch $A$ nach $A_{0}$, so ist $M^{\prime \prime}$ der Schnittpunkt von $O^{\prime \prime} S^{\prime \prime}$ mit der Mittelsenkrechten von $A_{0}^{\prime \prime} S^{\prime \prime}$. - Die vertikale Ebene durch $A B$ schneidet die Kugel in einem Kreise $k$, dessen Mittelpunkt $N$ von der Mitte $Q$ von $A B$ ebenso weit entfernt liegt, wie $M$ von $O$. Wir erhalten daher die Umlegung des Mauerbogens $A B$ in die $\Pi_{1}$, wenn wir auf der Geraden $O Q$ die Strecke $Q N_{0}=O^{\prime \prime} M^{\prime \prime}$ machen und um $N_{0}$ den Kreisbogen $k_{0}$ von $A$ bis $B$ beschreiben. Hieraus ergibt sich sofort die zweite Projektion $k^{\prime \prime}$. Wählen wir nämlich auf $k_{0}$ den Punkt $P_{\mathbf{0}}$ beliebig und ziehen $P_{0} P^{\prime} \perp A B$, so ist Abstand $P^{\prime \prime} x=P_{0}^{\prime} P^{\prime}$. Der Scheitel des Ellipsenbogens $k^{\prime \prime}$ liegt senkrecht über $Q$ und wird in derselben Weise wie der Punkt $P^{\prime \prime}$ gefunden. - Übrigens läbt sich die Ellipse $k^{\prime \prime}$ auch leicht aus ihren Achsen konstruieren.

87. Aufgabe. Die Eigenscbattengrenze einer Kugel und ihren Schlagschatten auf die Projektionsebenen für

Fig. 89 .
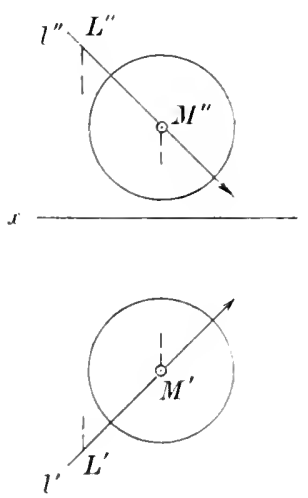

Parallelbeleuchtung zu konstruieren (Fig. 89). Wir bezeichnen wieder mit $M$ den Mittelpunkt, mit $u$ und $v$ den ersten und zweiten Umriß der Kugel, mit $l$ den durch $M$ gehenden Lichtstrahl. Die gesuchte Eigenschattengrenze ist ein Hauptkreis $s$, dessen Ebene auf $l$ senkrecht 
steht, ihre erste Projektion also eine Ellipse $s^{\prime}$ rom Mittelpunkt $M^{\prime}$. Da alle Durchmesser ron $s$ im Grundrib verkürzt erscheinen, mit Ausnahme des horizontalen, also in $u$ liegenden Durchmessers $A B$, so ist die auf $7^{\prime}$ senkrechte Strecke $A^{\prime} B^{\prime}$ die große Achse von $s^{\prime}$. Die kleine Achse ist demnach der (irundriß des Durchmessers ( $I$ ) von $s$, der sich in der ersten projizierenden Ebene von $l$ befindet. Diese schneidet die Kugel in einem Hauptkreise $w$, ron dem $l$ und $C D$ zwei anfeinander senkrechte Durchmesser sind. Um $C^{\prime} D^{\prime} z u$ konstruieren, drehen wir den Kreis $w$ un den vertikalen Kugeldurchmesser, bis er mit $v$ zuzammenfällt ${ }^{1}$ ). Dabei beschreibt der auf $l$ beliebig gewählte Punkt $I$. einen horizontalen Kreisbogen bis $L_{0}\left(M^{\prime} L_{0}^{\prime} \| x\right.$ und $\left.=M^{\prime} L^{\prime}, L^{\prime \prime} L_{0}^{\prime \prime} \| x\right)$. Damn ist $M^{\prime \prime} L_{0}^{\prime \prime}$ der Anfriß $l_{0}^{\prime \prime}$ des gedrehten Lichtstrahls; ziehen wir also in $I^{\prime \prime}$ den Radius $M^{\prime \prime} C_{0}^{\prime \prime} \perp l_{0}^{\prime \prime}$ als Aufrib der gedrehten Strecke $M C$, so entspricht dem Punkte $C_{0}^{\prime \prime}$ auf $v^{\prime}$ der Punkt $C_{0}^{\prime}$ und anf $l^{\prime}$ der Punkt $C^{\prime}\left(M^{\prime} C^{\prime}=M I^{\prime} C_{0}^{\prime}\right)$.

Der Aufriß von $s$ ist eine Ellipse, die den auf $7^{\prime \prime}$ senkrechten Durchmesser von $r$ zur großen Achse hat. Sie geht ferner durch den Punkt $A^{\prime \prime}$ auf $u^{\prime \prime}$, wodurch die Länge der kleinen Halbaclıse nach Art. 76 bestimunt ist.

Die Projektion der Eigenschattengrenze berübrt den scheinbaren Umrib in den Punkten, deren Tangenten zur Projektion des Lichtstrahls parallel sind. Dies gilt allgemein für jede krumme Fläche; denn trifft die Eigenschattengrenze $s$ einer solchen Fläche den wahren Umrib in $A$, so liegen der durch $A$ gehende Lichtstrahl und die zugehörigen Tangenten von $u$ und $s$ in der Berührungsebene von $A$, und da diese eine projizierende Ebene ist, so fällt ihre Projektion mit der des Lichtstrahls zusammen.

Die Grenze des Schlagschattens der Kugel auf die $\Pi_{1}$ ist der Schatten von $s$, also eine Ellipse $s_{h}$ vom Mittelpunkt $M_{h}$. 1)a die Durchmessser von $s$ auf der Lichtrichtung senkrecht stehen, so erscheinen sie im Schatten vergrößert, mit Arisnahme des zu $\Pi_{1}$ parallelen Durchmessers $A B$. Die kleine Achse von $s_{h}$ ist also $A_{h} B_{h} \# A^{\prime} B^{\prime}$, die grobe Achse folglich $C_{h} D_{h}$. Ziehen wir $C_{0}^{\prime \prime} N_{0}^{\prime \prime} \| l_{0}^{\prime \prime}$ bis zur Parallelen durch $M^{\prime \prime}$ zu $x$, so ist $M_{h} C_{h}=M^{\prime \prime} N_{0}^{\prime \prime}$. - Schneidet $s_{h}$ die Projektionsachse $x$, so fällt ein Teil des Kugelschattens auf die $\Pi_{2}$. Seine Grenze $s_{v}$ wird am einfachsten gefunden, indem man zu einer Reihe von Punkten $P_{h} \cdot Q_{h} \ldots$ des oberhalb $x$ liegenden 'Teils von $s_{h}$ die entsprechenden Punkte $P_{v}^{\prime}, Q_{v} \ldots$ konstruiert.

\section{Kegel- und Zylinderflächen.}

Raumkurven und abwickelbare Flächen im allgemeinen. Entstehung der Kegel- und Zylinderflächen.

88. l)ie 'Tangente in Punkte $P$ ' einer Raumkurve $k$ wird ebenso definiert, wie bei einer ebenen Kurve (Art. 71). Unter der Schmiegungs-

1) Wir könnten auch $w$ und $l$ auf eine $\Pi_{3}$ projizieren, parallel zur ersten projizierenden Ebene von $l$, oder wir könnten diese Ebene durch Drehung um den horizontalen Durchmesser vom $w \| \Pi_{l}$ machen, wodureh $w$ mit $u z u-$ sammentiele. 
ebene der Raumkurve $k$ in $I^{\prime}$ verstelit man die Grenzlage $\Sigma$, der sich die Verbindungsebene der Tangente in $P$ mit einem veränderlichen Kurvenpunkte nähert, wenn dieser dem Punkte $P$ zustrebt. Wir köunen die Schmiegungsebene auch definieren als die Ebene durch drei unendlich benachbarte Kurvenpunkte $P, P_{1}, P_{2}$ oder durch zwei unendlich benachbarte 'Tangenten $t=I^{\prime} Y_{1}^{\prime}$ und $t_{1}=P_{1} P_{2}$. Die Kurve $k$ durchsetzt die Ebene $\Sigma$ in Punkte $P$, weil dieser aus der Vereinigung von drei anfeinander folgenden Schnittpunkten herrorgeht. Eine Ausuahme machen nur solche singuläre Kurvenpunkte, in denen die Schmiegungsebene nicht bloß drei, sondern vier oder eine noch höhere gerade Anzahl von Punkten mit der Kurve gemein hat. - In $\Sigma$ befindet sich der Kr ümmungskreis des Punktes $I^{\prime}$, d. h. der Kreis durch $I^{\prime}, P_{1}, I_{2}^{\prime}$.

Verzeichnen wir auf $k$ von $P_{2}$ aus weiter die unendlich benachbarten Punkte $P_{3}, P_{4} \ldots$, so sind die Geraden $t_{2}=P_{2} P_{3}, t_{3}=I_{3} P_{4} \ldots$ die Tangenten von $k$ in $P_{2}, P_{3} \ldots$, und dann bilden die unendlich schmalen ebenen Streifen, die zwischen je zwei Nachbartangenten enthalten sind, in ihrer stetigen Aufeinanderfolge eine gewisse Fläche, die Tangentenfläche der Raumkurve $k$ (Fig. 90). Sie ist abwickelbar, d. h. wir können sie ohne Falten, Dehnen oder Zerreißen in eine Ebene ausbreiten, wie sich sofort ergibt, weun wir den ersten Elementarstreifen $t t_{1}$ durch eine unendlich kleine Drehung um $t_{1}$ in die Ebene des folgenden $t_{1} t_{2}$ bringen, dann beide vereinigt in die Ebene des dritten überführen usw.

Die Tangenten der Raumkurve heißen

Fig. 90 .

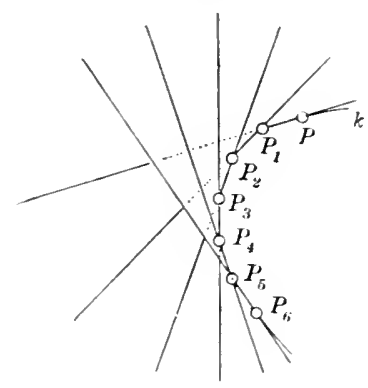
die Erzeugenden oder Mantellinien ihrer

Tangentenfläche. Ihre Schmiegungsebenen sind die Berührungsebenen der Fläche, und zwar berührt jede von ihnen die Fläche längs der zugehörigen Erzeugenden; denn jede Gerade, die z. B. in der Schmiegungsebene $\Sigma$ beliebig gezogen wird, hat mit der Fläche die beiden unendlich benachbarten Punkte gemein, in denen sie $t$ und $t_{1}$ schneidet, ist also eine Tangente der Fläche.

Die Tangentenfläche besteht aus zwei Mänteln, die sich längs der Raumkurve $l$ berühren; diese bildet daher eine scharfe Kante der Fläche, die sogenannte Rückkehrkante. Jede durch einen Punkt von $k$, aber nicht durch seine Tangente gehende Ebene schneidet die Fläche in einer Kurve, die in ihm einen Rückkehrpunkt (eine Spitze) hat.

89. Durch Bewegung einer Geraden entsteht eine geradlinige Fläche oder Regelfläche. Bewegt sich die erzeugende Gerade so, daß je zwei unendlich benachbarte Lagen sich schneiden, so ist die Fläche abwickelbar (Art. 88); im entgegengesetzten Falle heißt sie windschief.

Eine Kegelfläche entsteht, wenn eine (unbegrenzte) Gerade sich so bewegt, daß sie beständig durch einen festen Punkt $S$ geht und eine gegebene Kurve $k$ fortwährend schneidet. Die sämtlichen Lagen der erzeugenden Geraden heißen Ilantellinien: $S$ wird die Spitze oder der Mittelpunkt, $k$ die Leitkurve der Kegelfläche genannt. 
Rückt der Punkt $S$ in unendliche Entfernung, bleibt also die Erzeugende zu ihrer Anfangslage parallel, so verwandelt sich die Kegelthiche in eine $/ y$ linderfläche.

Jede Kegel- oder Zylinderfläche kann als der Mantel einer Pyramide bzw. eines Prismas mit unendlich vielen, unendlich schmalen Seitenflächen anfgefabt werden. Sie ist also abwickelbar und wird von jeder Berïhrungsebene in allen Punkten einer Mantellinie berührt. Aus dieser Auffassung ergibt sich ferner: Alle Parallelschnitte einer Kegelfläche sind einander ähnlich. Alle Parallelschnitte einer Zylinderfläche sind kongruent.

\section{Darstellung der Zylinder-und Kegelflächen.}

Berührungsebenen. Schattenkonstruktionen. Schnittpunkte mit einer Geraden.

90. Darstellung eines schiefen Kreiszylinders, dessen Grundkreis $k$ in $\Pi_{1}$ liegt, und dessen Mantellinien zu einer gegebenen Geraden parallel sind, z. B. zu der durch den Mittelpunkt $M$ von $k$ gehenden Geraden $M N$ (Fig. 91). Die

Fig. 91.
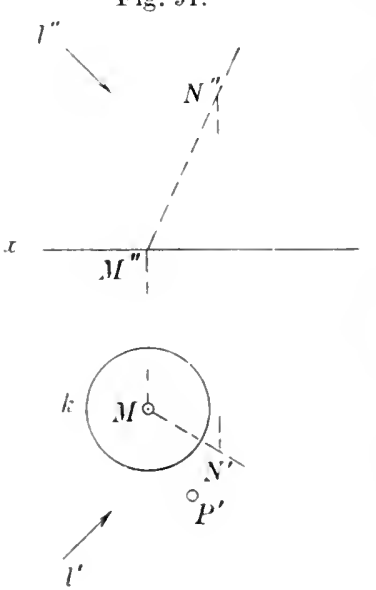

Mantellinien, deren Berührungsebenen auf $\Pi_{1}$ senkrecht stehen, bilden den ersten wahren Umrib; ihre ersten Projektionen sind Tangenten an $k \| M N^{\prime}$. Die zweiten wahren Umrißlinien gehen durch die Endpunkte des zu $x$ parallelen Durchmessers von $k$.

Um zu einem Punkte $P$ der Zylinderfläche, von dem der Grundrib $P^{\prime}$ gegeben ist, den Aufriß zu ermitteln, ziehen wir durch $P^{\prime}$ die Gerade $p^{\prime} \| M N^{\prime}$ und zeichnen im Aufrib die beiden Mantellinien, die im Grundrif mit $p^{\prime}$ zusammenfallen. - Die Berührungsebene der Zylinderfläche in $I^{\prime}$ geht durch die zugehörige Mantellinie; ihre Grundrißspur berührt $k$ im ersten Spurpunkte dieser Geraden.

Die Eigenschattengrenze des Zylinders für Licht in der Richtung $l$ wird durch die Mantellinien gebildet, deren Berührungsubenen $\| l$ sind. Wir ermitteln sie aus dem Schlagschatten, den der Zylinder auf die $\Pi_{1}$ wirft: Konstruieren wir von $\| N$ den Schatten $I N_{h}$, so ist der Schatten jeder Mantellinie zu $M N_{h}$ parallel. Ziehen wir also in dieser Richtung an $k$ die 'Tangenten $e_{h}$ und $f_{h}$, so luaben wir rlamit die Grenzen des Zylinderschattens gefunden; ihnen ent-prechen als Eigenschattengrenzen die Mantellinien $e$ und $f$, die durch die Berührungspunkte der beiden Tangenten gehen.

Bei einem geraden Kreiszylinder, clessen Achse auf $\Pi_{1}$ senkrecht steht, sind die Geraden $e_{h}$ und $t_{h} \| l^{\prime}$. Ist daher die Achse $M N \| \Pi_{1}$, aber gegen $\Pi_{2}$ heliebig geneigt, so erhalten wir die Eigenschattengrenzen $e$ und $f$ mit Hilfe einer $\Pi_{3} \perp M N$. 'Tangenten $\| l^{\prime \prime \prime}$ an die 
dritte Projektion des Grundkreises bestimmen nämlich auf diesem die Anfangspunkte der Mantellinien $e$ und $f$.

Um einen geraden Kreiszylinder, dessen Grundkreis $k$ in $\Pi_{1}$ lient, in schiefer Projektion auf die $\Pi_{2}$ zu zeichnen, denken wir uns $k$ durch seine Unlegnng $k_{0}$ in die $\Pi_{2}$ gegeben nnd konstruieren zum Mittelpunkte $M_{0}$ das Bild $M_{s}$ (vgl. Art. 4). Dann ist die Bildellipse $k_{s}$ perspektiv affin zu $k_{0}$ mit $x$ als Affinitätsachse, wir finden also ihre Achsen nach der in Art. 73 gelösten Grundaufgabe. Die affine Beziehung zwischen $k_{0}$ und der noch nicht gezeichneten Ellipse $k_{s}$ dient auch zur Konstruktion der scheinbaren Umriblinien des Zylinders sowie seines Schattens anf die $\Pi_{1}$ und seiner Eigenschattengrenzen (vgl. Art. 75).

91. Narstellung eines schiefen Kreiskegels, dessen Grundkreis $k$ in $\Pi_{1}$ liegt (Fig. 92). Ist $S$ die Spitze des Kegels, so besteht

Fig. 92.

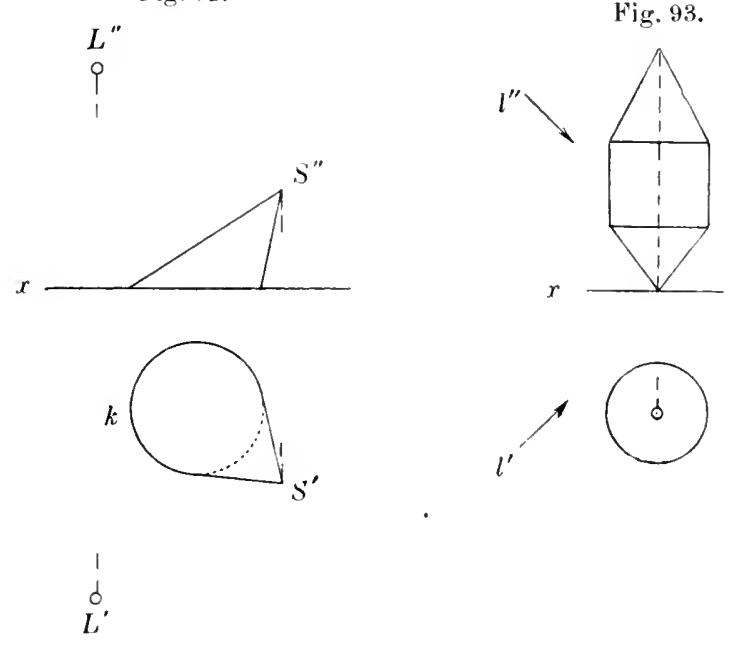

sein erster scheinbarer Umrib aus den Tangenten von $S^{\prime}$ an $k$. llieser Umriß ist also nicht vorhanden, wenn $S^{\prime}$ innerhalb $k$ liegt; dann hat nämlich der Kegel keine auf $\Pi_{1}$ senkrechten Berührungsebenen.

Die Berührungsebenen des Kegels aus dem gegebenen Pnnkte $L$ gehen durch die Gerade $L S$, die $\Pi_{1}$ in $S_{h}$ schneidet; ihre Grundribspuren sind also die Tangenten ans $S_{h}$ an $k$. Die zugehörigen Berührungsmantellinien bilden die Eigenschattengrenze des Kegels für Beleuchtung aus $L$.

Um die Eigenschattengrenze des in Fig. 93 dargestellten Drehkörpers, der aus einem Zylinder und zwei Kegeln bestelıt, für Licht in der Richtung $l$ zu konstruieren, benutzt man die Schnittpunkte der durch die Spitzen gehenden Lichtstrahlen mit den Ebenen der zugehörigen Grundkreise. Man beachte, daß die Eigenschattengrenzen der Teilkörper keine gemeinsamen Punkte besitzen.

92. Anfgabe. Die Schnittpunkte $P$ und $Q$ der Geraden $y$ mit einer Kegelfläche zu ermitteln, deren Grundkreis $k$ in $\Pi_{1}$ 
liegt (Fig. 94). Nach Art. 5! und 62 legen wir durch $g$ und durch die spitze $S$ des Kegels eine Hilfsebene $\Delta$. Z Zu dem $/$ wecke ziehen wir durch $S$ die Gerade $h \| l g$ und bestimmen von $q$ und $h$ die ersten Spur-

Fig. 94
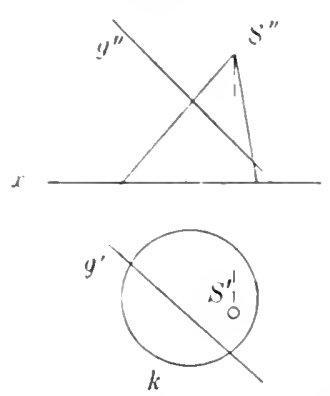
punkte $G_{1}$ und $I I_{1}$, sowie ron $\Delta$ die Spurlinie $G_{1} H_{1}$. Sind $A$ und $B$ die Schnittpunkte vou $k$ mit $G_{1} H_{1}$, so schneidet $\Delta$ die Kegelfliiche in den Mantellinien $S A$ und $S B$, und diese treffen $g$ in $I^{\prime}$ und $Q$.

Tritt an die Stelle des Kegels ein $7 y-$ linder, so legen wir die Ebene $\Delta$ durch $g$ parallel zu den Mlantellinien, ziehen also durch einen beliebigen Punkt von $g$ zu den Mantellinien eine Parallele usw.

93. Darstellung eines geraden Kreiskegels, ron dem diespitze $S$, sowie der Mittelpunkt $M$ und der Radius $r$ des (irundkreises $k$ gegeben sind (Fig. 95). Wir konstruieren die Umriblinien des Kegels mit Hilfe der ihm im lireise $k$ eingeschriebenen Kngel. Jede Ebene, die den Kegel längs einer Mantellinie berührt, berührt auch die Kugel im Schnittpunkte der Mantellinie mit $k$. Ist nun S' $T$ eine C'mriblinie des Kegels, z. B. eine erste, und $T$ ibr Schnittpunkt mit $k$, so steht ihre Berührungsebene $\perp \Pi_{1}$. Dann liegt aber $T$ auch auf dem ersten Umribkreise $u$ der Kugel, folglich berührt im

Fig. 95 .

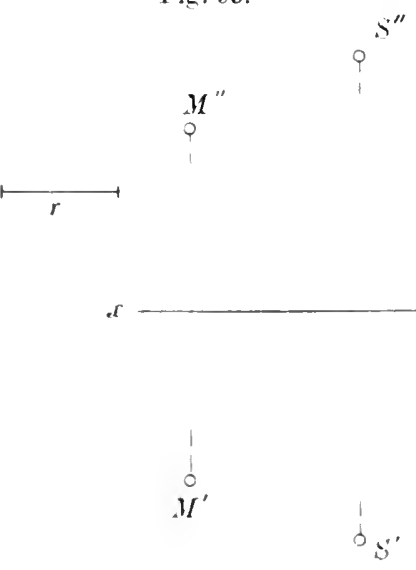

Grundriß die Gerade $S^{\prime \prime} T^{\prime \prime}$ den Kreis $u^{\prime}-$ und die Ellipse $k^{\prime}-$ in $T^{\prime}$. Die scheinbaren Umrißlinien des Kegels sind daher die Tangenten aus $S^{\prime}$ und $S^{\prime \prime}$ an die scheinbaren Umrisse der Hilfskugel.

Ansführung: Die erste projizierende Ebene von $S M I$ schneidet den durch $k$ begrenzten Kegel in einem gleichschenkligen Dreieck C'S D. Legen wir sie in die $\Pi_{1}$ um, so gelangt $S M$ nach $S_{0} \lambda U_{0}$ und $C$ nach $C_{0}$; dabei ist $M I^{\prime} M_{0} \perp S^{\prime} M I^{\prime}$ nnd $=$ Abstand $M^{\prime \prime} x$, $M_{0} C_{0} \perp S_{0} M_{0}$ und $=r$. Ziehen wir dann $C_{0} O_{0} \perp S_{0} C_{0}$ bis $S_{0} M I_{0}$, so ist $O_{0}$ die Unlegung des Mittelpunkts der Hilfskngel und $O_{0} C_{0}$ ihr Radius; ihre scheinbaren Umrisse sind also die

Kreise ${ }^{\prime}$ und $v^{\prime \prime}$ um $O^{\prime}$ und $O^{\prime \prime}$ mit dem Radius $O_{0} C_{0}$. An diese legen wir ans $S^{\prime}$ und $S^{\prime \prime}$ die Tangentenpaare $S^{\prime} T^{\prime \prime}, S^{\prime} U^{\prime}$ und $S^{\prime \prime} V^{\prime \prime}$, $S^{\prime \prime} W^{\prime \prime}$. - Die Projektionen des Grundkreises $k$ werden wie in Art. 79 gefunden.

Schnitt einer Zylinderfläche mit einer Ebene.

94. Aufgabe. Den śchnitt eines geraden Kreiszylinders, dessen Grundkreis $k_{1}$ in $\Pi_{1}$ liegt, nit einer auf $\Pi_{2}$ senk- 
rechten Ebene $E\left(e_{2} e_{2}\right)$ zn konstruieren (Fig. 96). Die Schnittkurve ist die Parallelprojektion des Grundkreises auf $E$, also eine Ellipse $l_{3}$, deren Mittelpunkt $M_{3}$ sich auf der Zylinderachse $M_{1} M_{2}$ befudet; ihre zweite Projektion ist die auf $e_{2}$ liegende Strecke $A_{3}^{\prime \prime} B_{3}^{\prime \prime}$ zwischen den scheinbaren Lmriblinien $A_{1}^{\prime \prime} A_{2}^{\prime \prime}$ und $b_{1}^{\prime \prime} B_{2}^{\prime \prime}$. Da $l_{3}^{\prime}$ mit $k_{1}$ zusammenfällt, so entspricht jedem Durchmesser von $k_{1}$ ein gröluerer Durchmesser von $k_{3}$, mit Ausnahme des zu $e_{1}$ parallelen Durchmessers

Fig. 96.

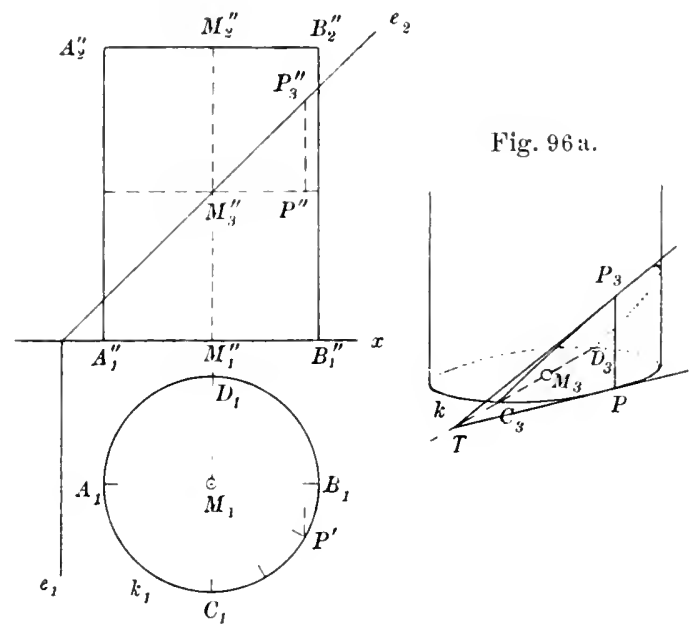

$C_{1} D_{1}$, dem in $k_{3}$ eine parallele und gleich große Strecke $C_{3} D_{3}$, also die kleine Achse zugeordnet ist. Als große Achse ergibt sich demnach $A_{3} B_{3} \# A_{3}^{\prime \prime} B_{3}^{\prime \prime}$.

Abwickelung. Schneiden wir den Zylinder nach der Mantellinie $C_{1} C_{2}$ auf und wickeln ihn in die Zeichenebene ab, so verwandelt er sich in eiu Rechteck, dessen Grundlinie $C_{1} C_{1}$ gleich dem Umfange von $k_{1}$ und dessen Höhe $=C_{1} C_{2}$ ist $^{1}$ ).

Die Ellipse $k_{3}$ verwandelt sich in der Abwickelung in eine aus vier kongruenten Teilen $C_{3} B_{3}, B_{3} D_{3} \ldots$ bestehende Kurve. $\mathrm{Um}$ sie zu koustruieren, legen wir durch $C_{3} D_{3}$ eine horizontale Ebene $\Sigma$, die den Zylinder in einem Kreise $k$, die Umriblinien $A_{1} A_{2}$ und $B_{1} B_{2}$ in $A$ und $B$ schneidet, und teilen den Quadranten $C_{3} B$, im Grundriß also den Quadranten $C_{2} B_{1}$ und in der Abwickelung die Strecke $C_{3} B$, in eine hinreichende Anzahl gleicher Teile. Ist $P$ ein solcher 'Teilpunkt,

1) Näherungsverfahren fïr die Rektifikation des Kreises $k_{1}$ : Wir ziehen durch $\boldsymbol{M}_{1}$ eine Gerade, die mit $\boldsymbol{M}_{1} C_{1}$ einen Winkel von $30^{\circ}$ bildet, schnoiden sie in $V^{*}$ mit der Tangente des Punktes $C_{1}^{*}$ und tragen auf dieser von $V$ aus in der Richtung nach $C_{1}$ den Padius $r$ des Kreises dreimal ab. Bezeichnet $U$ den Endpunkt der so erhaltenen Strecke, so ist

$$
\frac{U D_{1}}{r}=3,14153 \ldots
$$


$P_{3}$ der Schnittpunkt von $\mathrm{E}$ mit der durch $I^{\prime}$ gehenden Mantellinie.

so machen wir in der Abwickelung die Strecke $P P_{3} \| C_{1} C_{2}^{\prime}$ und $=P^{\prime \prime} P_{3}^{\prime \prime}$.

Die 'Tangente in $P_{3}$ an die Schnittellipse $F_{3}$ ist die schnittlinie der Ebene E mit der Berührungsebene des Zylinders in $I_{3}^{\prime}$. Die Beriihrungsebene schneidet $\Sigma$ in der 'Tangente $I^{\prime} I$ ' von $k$, und diese trifft $C_{3} D_{3}$ im Punkte $T$, dessen Grundriß ' $T^{\prime}$ mit dem schnittpunkte von $C_{1}^{\prime} I_{1}$ und der 'Tangente in $P^{\prime}$ an $k_{1}$ zusammenfällt; dann ist $P_{3} T$ die Tangente au $k_{3}$. (Fig. 96 a gibt hierzu eine Skizze in schiefer Parallelprojektion.) - Wie Tangente an die verwandelte Kurve bildet mit der Geraden $P_{3} P$ denselben Winkel, wie die Ellipsentangente mit der entsprechenden Mantellinie; wir erhalten sie also, indem wir das rechtwinklige Dreieck $P_{3}^{\prime} P^{\prime} T$ in der Abwickelungsfigur an $P_{3} I^{\prime}$ wieder anheften. $\mathrm{Zu}$ dem $/$ wecke machen wir auf $P C_{3}$ die Strecke $I^{\prime} T=I^{\prime} T^{\prime}$.

Wählen wir in der Abwickelung den Punkt $C_{3}$ zum Anfangspunkte eines rechtwinkligen hoordinatensystems mit der $x^{-A}$-Ahse $C_{3} D_{3}$ und bezeichnen mit $\mathfrak{x}, \mathfrak{y}$ die Koordinaten von $I_{3}^{\prime}$, mit $\varphi$ den Winkel $P^{\prime} M_{1} C_{1}$, mit $\varepsilon_{1}$ den ersten Neigungswinkel von $E$, so ist $æ$ gleich dem Bogen $G_{1}^{\prime} P^{\prime}$ von $k_{1}$, also $=r \varphi$. Dann folgt aus $\Delta P_{3}^{\prime \prime} P^{\prime \prime} M_{3}^{\prime \prime}$

$$
\mathfrak{y}=I_{3}^{\prime \prime} I^{\prime \prime}=H_{3}^{\prime \prime} P^{\prime \prime} \cdot \operatorname{tg} \varepsilon_{1}=r \sin \varphi \operatorname{tg} \varepsilon_{1}=r \operatorname{tg} \varepsilon_{1} \sin \frac{\mathfrak{r}}{r} \text {. }
$$

Die Verwandelte der Schnittellipse ist also eine Sinus- kurve mit den Scheiteln $A_{3}, B_{3}$ und den Wendepunkten $C_{3}, D_{3}$. Die Inflexionstangenten in $C_{3}$ und $D_{3}$ bilden mit $C_{3} D_{3}$ denselben Winkel wie die Ellipsentangente in $C_{3}$ mit der Tangente von $k$, d. h. den Winkel $\varepsilon_{1}$.

95. Beziehung zwisclen dem Krünmungsradius $\varrho$ in irgend einem Punkte $P$ einer a f einer abwickelbaren Fläche liegenden Kurve $c$ und dem Krümmungsradius $\varrho_{0}$ im ent-

Fig. 97 .

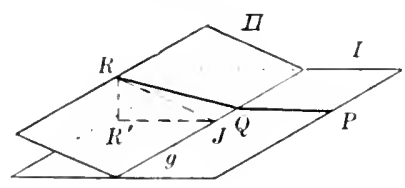
sprechenden Punkte der verwandelten Kurve $c_{0}$ (Fig.97). Wir denken uns die abwickelbare Fläche wie in Art. 88 in unendlich schmale ebene Streifen zerlegt und bezeichnen mit I und II zwei aufeinanderfolgende Streifen, die sich in der Geraden $g$ schneiden, mit $P Q$ und $Q R$ die in ihnen liegenden Elemente der gegebenen Kurve $c$. Der durch $I^{\prime}, Q$ und $R$ bestimmte Krois ist der Krümmungskreis $*$ von $c$ in $P$, seine Ebene die zugehörigre Schmiegungsphene $E$, falls $c$ nicht ganz in dieser Ebene liegt. Drehen wir den Streifen II unendlich wenig um $g$, his or in die Erweiterung der Ebene I gelangt, so komnit $R$ nach $R_{0}$, und dann sind $P, Q$ und $R_{0}$ drei Punkte ler verwandelten hurve $c_{0}$. Aber $R_{0}$ fällt mit der senkrechten Projektion $l^{\prime}$ ron $R$ auf die Ebene I zusammen. Bezeichnen wir nämlich mit $J$ den Fubpunkt des Lotes von $R$ auf $/$, so liegt $R_{0}$ auf der Geraden $l^{\prime} J \perp g$, und es ist $R_{0} J=R J$, also von unendlich kleinen Größen höherer Ordnung abgesehen $=R^{\prime} \cdot J$, weil in dem rechtwinkligen Dreieck $I i J R^{\prime}$ der Winkel bei $J$ als Neigungswinkel der Ebenen I und II 
unendlich klein ist. Der Krümmungsradius $\varrho_{0}$ der Kurve $c_{0}$ in $P$ ist demnach gleich dem Krümmungradius der senkrechten Projektion Fon $c$ auf die Ebene I, d. h. auf die Berührungsebene der abwickelbaren Fläche in $P$.

Die Kurre $c$ kann uun von $I^{\prime}$ bis $I i$ durch ihren Krümmungskreis $*$ ersetzt werden, folglich ist $\rho_{0}$ auch gleich dem Krümmungsradius der senkrechten Projektion von $*$ auf $I$, d. i. eine Ellipse $\boldsymbol{x}^{\prime}$, die * in $P$ berührt, mit $I^{\prime}$ als Endpunkt der kleinen Achse. Berleutet $\%$ den Neigungswinkel der Ebenen I und $E$, so ist die kleine Halbachse von $\varkappa^{\prime}=\rho \cos \varkappa$ und die große Halbachse $=\rho$, also der Krümmungsradius im Scheitel $P=\rho^{2}: 0 \cos \alpha$. Hieraus ergibt sich für deu Krümmungsradius der verwandelten hurve $c_{0}$ der einfache Ausdruck

$$
\varrho_{0}=\frac{\varrho}{\cos u}
$$

Dabei bezeichnet $\alpha$ den Winkel, den im Punkte $P$ die Berührungsebene der abwickelbaren Fläche und die Schmiegungsebene von $c$ (bzw. die Ebene dieser Kurve) miteinander bilden.

Für $\%=90^{\circ}$ wird $\varrho_{0}=\infty$, und dann hat die Kurve $c_{0}$ in $P$ in der Regel einen Weudepunkt, unter Umständen auch einen Flachpunkt (vgl. Art. 71). Ist $c$ eine ebene Kurve, so ergibt sich hieraus der Satz: Schneidet man eine abwickelbare Fläche mit einer Ebene, so verwandeln sich diejenigen Punkte der Schnittkurve, deren Berührungsebenen auf der schneidenden Ebene senkrecht stehen, im allgemeinen in Wendepunkte.

Anwendung auf die in Art. 94 behandelte Aufgabe. Die Punkte $C_{3}$ und $D_{3}$ der Schnittellipse $l_{3}$ werden zu Wendepunkten der verwandelten Kurve, denn die zugehörigen Berührungsebenen des Zylinders sind $\| \Pi_{2}$, also $\perp E$. - Gleichung 1) liefert eine einfache Konstruktion der Krümmungskreise in den Scheiteln $A_{3}$ und $B_{3}$ der Sinuskurve $k_{3}$ : Auf dem Zylinder sind $A_{3}$ und $B_{3}$ die Endpunkte der großen Achse der Schnittellipse; für beide ist $\psi=90^{\circ}-\varepsilon_{1}$ und nach Art. 77 $\varrho=\frac{M_{3} A^{2}}{M_{3} A_{3}}$, folglich nach Gleichung 1)

$$
\varrho_{0}=\frac{M_{3} A^{2}}{M_{3} A_{3} \sin \varepsilon_{1}}=\frac{M_{3} A^{2}}{A A_{3}} .
$$

Ziehen wir also in Fig. $96 M_{3}^{\prime \prime} F \perp e_{2}$ bis $A_{1}^{\prime \prime} A_{2}^{\prime \prime}$, so ist $A^{\prime \prime} F$ der Krümmungradius der Sinuskurve $k_{3}$ in $A_{3}$ und $B_{3}$.

86. Befindet sich die den Zylinder schneidende Ebene in beliebiger Lage gegen die Projektionstafeln, so nehmen wir eine $\Pi_{3} \perp e_{1}$ zu Hilfe and konstruieren aus Grund- und Seitenrib wie vorher die Achsen $A_{3} B_{3}$ und $C_{3} D_{3}$ der Schnittellipse $k_{3}$, sowie die Abwickelung des Zylinders. Nach Art. 72 ist die Parallelprojektion von $k_{3}$ eine Kurse zweiter Ordnung, die sich im Endlichen schließt, d. h. wieder eine Ellipse, und zwar entsprechen zwei konjugierten Durchmessern von $k_{3}$ auch im Bilde zwei solche Durchmesser, denn jeder ron ihnen bleibt parallel zu den Tangenten in den Endpunkten des anderen. Demnach sind im Aufriß 
die l'rojektionen von $A_{3} B_{3}$ und $C_{3} D_{3}$ zwei konjugierte Durchmesser der Ellipse $k_{3}^{\prime \prime}$, deren Achsen hierlurch bestimint werden (Art. 7S). Noch kürzer erhalten wir diese Achsen nach Art. 73 auf Grund der perspektiv affinen Beziehung zwischen den Projektionen $k_{1}$ und $k_{3}$ der schnittellipse $k_{3}$ (Art. 52). Dabei besteht die Affinitätsachse aus den. sich deckenden Projektionen der Schnittlinie von $E$ mit der zweiten Halbierungsebene. Wir konstruieren sie als Verbindungshinie des Punktes $e_{1} \times e_{2}$ mit dem Schnittpunkte von Grund- und Aufriß irgend einer in $\mathrm{E}$ liegenden Geraden, z. B. der zwoiten Hauptlinie $n$ in der.

Fig. 98.

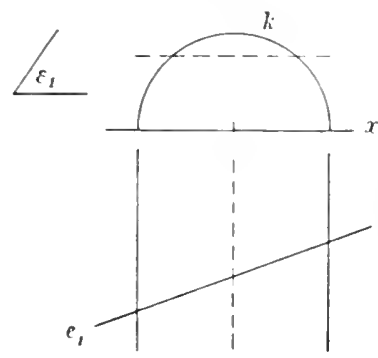
durch $M_{1} M_{2} \| \Pi_{2}$ gelegten Ebene. - Die. Gerade $n$ gelit durch den Mittelpunkt $\boldsymbol{M}_{3}$ und durch die Schnittpunkte von $k_{3}$ mit dem. zweiten Uinriß des Zylinders.

97. Häufig konstruiert man die Schnittellipse nur punktweise, also ohne ihre Achsen zu bestimmen. Als Beispiel betrachten wir in Fig. 98 einen halben geraden Kreiszylinder, dessen Leitkurve der in $\Pi_{2}$ über $x$ liegende Halbkreis $k$ ist, und bestimmen die Ebene $\mathbf{E}$ durch ihre spur $e_{1}$ und die erste Tafelneigung $\varepsilon_{1}$. (Zylindrischer Durchgang durch eine schräge Böschungsmauer.) Um hier die Schnittkurve zu ermitteln, verwenden wir horizontale Hilfsebenen. Eine solche Ebene schneidet den Zylinder in zwei Mantellinien und $E$ in einer Parallelen zu $e_{1}$, deren Grundrif nach Art. 35 konstruiert wird; diese Gerade trifft die Mantellinien in Punkten. der gesuchten Schnittellipse.

98. Um den Schnitt der Ebene $E\left(e_{1} e_{2}\right)$ mit einem schiefen Kreiszylinder zu konstruieren, dessen Grundkreis $l_{1}$ in $\Pi_{1}$ liegt, suchen wir zuerst den Schnittpunkt $\|_{3}$ von $E$ mit der Parallelen zu den Mantellinien durch den Mittelpunkt $\boldsymbol{M}_{1}$ von $k_{1}$. Die Schnittkurve. ist eine Ellipse $k_{3}$ rom Mittelpunkte $H_{3}$, nämlich die perspektiv affine Kurve zu $k_{1}$ mit $e_{1}$ als Affinitäsachse und $M_{1}, M_{3}$ als einem Par entsprechender Punkte; je zwei aufeinander senkrechten Durchmessern von $l_{1}$ entsprechen also in $k_{3}$ zwei konjugierte Durchmesser. - Liegt $l_{1}$ nicht in $\Pi_{1}$, so tritt an die Stelle von $e_{1}$ als Affinitätsachse die Schnittlinie von $E$ mit der Ebene von $k_{1}$.

99. Abwickelung eines halben schiefen Kreiszylinders, der durcb die in $\Pi_{1}$ liegrenden Mantellinien $A_{1} A_{2}$ und $B_{1} B_{2}$, sowie durch die vertikalen Halbkreise $l_{1}$ und $h_{2}$ über $A_{1} B_{1}$ und $A_{2} B_{2}$ begrenzt ist (Fig. 99). Wie bei der Abwickelung eines schiefen Prismas (Art. 5\%) benutzen wir einen Normalschnitt: Eine Ebene $\perp A_{1} A_{2}$ schneidet den Halbzylinder in einer halben Ellipse $k_{3}$ mit der kleinen Achse $A_{3} B_{3}$; die grobe Halbachse ist gleich dem Radius $r$ von $k_{1}$. Darauf teilen wir $k_{1}$ - zuerst in rer Umlegung $k_{1}^{0}$ in $\Pi_{1}$ - in eine grenügende Anzahl, z. B. 12, gleicher Teile, ziehen durch die 'Teilpunkte $1_{1}, 2_{1} \ldots$ im Grundrib die Mantellinien $1_{1} 1_{2}$, $2_{1} 2_{2} \ldots$ und bestimmen ihre Schnittpunkte $1_{3}, 2_{3} \ldots$ mit $k_{3}$, sowie auf ${ }^{-}$ 
der Umlegung $k_{3}^{0}$ vou $k_{3}$ die entsprechenden Punkte $1_{3,}^{0}, 2_{3,}^{0} \ldots$; dabei ist $1_{1}^{0} 1_{1} \perp A_{1} B_{1}, 1_{1}^{\prime} 1_{2} \|^{3} A_{1} A_{2}, 1_{3}^{\prime} 1_{3}{ }^{0}=1_{1}^{\prime} 1_{1}{ }^{0}$ usw. Halten wir die Mantellinie $A_{1} A_{2}$ fest und wickeln den Zylinder mit seiner Außenseite nach unten in die Grundribebene ab, so fallen die Punkte $1_{3}, 2_{3} \ldots$ auf die Verlängerung von $B_{3} A_{3}$, und wir finden ihre nenen Lagen, indem wir die ungleichen Bogrenstücke der

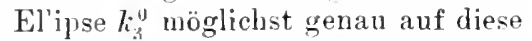
Gerade übertragen. Durch die so erhaltenen Punkte ziehen wir die abgewickelten Mantellinien, z. B. durch $1_{3}$ die Gerade $1_{3} 1_{1} \# 1_{3}^{\prime} l_{1}^{\prime}$; dann ist $1_{1}$ ein Punkt der Verwandelten des Halbkreises $l_{i}$.

Die Verbindungslinie von $1_{1}$ mit $l_{1}^{\prime}$ ist $\perp A_{1} A_{2}$ und der Bogen $A_{1} 1_{1}$ der Verwandelten $=\frac{1}{12}$ von $k_{1}$. Ersetzen wir daher den Zylinder durch das eingeschriebene Prisma Fig. 99.

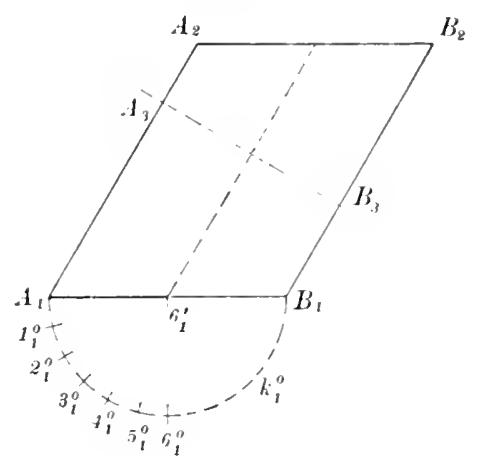
mit der Grundfläche $A_{1} 1_{1} 2, \ldots B_{1}$, so gelangen wir zu folgender Näherungskonstruktion ohne Zuhilfenahme eines Normalschnitts: Wir fällen von den Punkten $1_{1}^{\prime}, 2_{1}^{\prime} \ldots$ auf $A_{1} A_{2}$ die Lote $1_{1}^{\prime} 1_{1}, 22_{1}^{\prime} \ldots$ und machen die Strecken $A_{1} 1_{1}, 1_{1} 2_{1} \ldots$ $=A_{1} 1_{1}^{0}$.

Die Verwandelte von $k_{1}$ hat in $6_{1}$ einen Wendepunkt, weil die Berührungsebene des Zylinders in diesem Punkte auf der Ebene von $k_{1}$ senkrecht steht (Art. 95). Die Inflexionstangente bildet mit der Geraden $6_{1} 6_{2}$ denselben Winkel, wie die Kreistangente in $6_{1}$ mit der entsprechenden Mantellinie, d. h. den Winkel $A_{2} A_{1} B_{1}$. 一 Der Krümmungsradius der Verwandelten im Scheitelpunkte $A_{1}$ ist nach Gleichung 1 ) in Art. $9 \check{y}=\frac{r}{\cos -A_{2} A_{1} B_{1}}$; der zugehörige Krümmungsmittelpunkt ist daher der Schnittpunkt von $A_{1} A_{2}$ mit der Uittelsenkrechten von $A_{1} B_{1}$.

Schnitt einer Kegelfläche mit einer Ebene.

100. Der Schnitt eines Kreiskegels mit einer nicht durch die Spitze gehenden Ebene ist eine Kurve zweiter Ordnung, weil jede Gerade der Ebene mit der Kegelfläche zwei (reelle oder imaginäre) Punkte gemein hat (Art. 92). Die Schnittkurve ist eine Ellipse (oder ein Kreis), eine Parabel oder Hyperbel, je nachdem die schneidende Ebene zu keiner, einer oder zwei Mantellinien parallel ist. $\mathrm{Cm}$ zu entscheiden, welcher dieser drei Fälle vorliegt, lege man durch die Spitze des Kegrels eine Ebene parallel zur schneidenden Ebene.

Die Parallelprojektion eines jeden der drei Kegelschnitte ist wieder eine Kurve zweiter ()rdnung (Art. 72), und zwar von derselben Art, weil den unendlich fernen Pnnkten des Kegelschnitts (und nur diesen) unendlich ferne Bilder entsprechen. 
101. Aufgabe. Den Schnitt der Ebene $E\left(e_{1} e_{2}\right)$ mit einem geraden Kreiskegel zu koustruieren, dessen Grundkreis $k$ in $\Pi_{1}$ liegt. Wir bezeichnen nit $S$ die Spitze des Kegels, mit $M$ den Nittelpunkt von $k$. Die libene, die durch $S M \perp e_{1}$ gelegt wird, teilt

Fig. 100 .

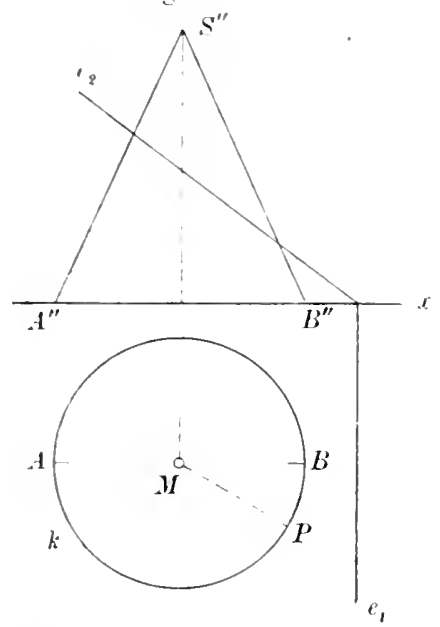
die Sehnittkurve $k_{1}$ in zwei symmetrische Hälften und enthält demnach eine Achse von $k_{1}$. Sie schneidet $E$ in einer Fallinie $f$, den Kegel in zwei Mantellinien $S A$ und $S B$, und diese bestimmen auf $f$ dio Achsenendpunkte $A_{1}$ und $B_{1}$. - Nehmen wir der Einfachheit wegen $E \perp \Pi_{2}$, so ist jene Symmetrieebene $\| \Pi_{2}$, und die zugehörigen Mantellinien $S A, S B$ bilden den zweiten Umrib des Kegels.

a) Elliptischer Schnitt (Fig. 100). Der Aufriß der Schnittellipse $k_{1}$ fällt mit der auf $e_{2}$ liegenden Strecke $A_{1}^{\prime \prime} B_{1}^{\prime \prime}$ zusammen. Die zweite Ellipsenachse geht durch den Mittelpunkt $O_{1}$ von $A_{1} B_{1}$ senkrecht zu $\Pi_{2}$; ihre Endpunkte $C_{1}$ und $D_{1}$ liegen auf dem Kreise $i$, in dem die durch $O_{1}$ gelegte Horizontalebene den Kegel schneidet. - Der Grmudrib von $k_{1}$ ist eine Ellipse mit den Achsen $A_{1}^{\prime} B_{1}^{\prime}$ und $C_{1}^{\prime} D_{1}^{\prime}$.

Bezeichnen wir mit $v$ und $w$ die Radien der durch $A_{1}$ und $B_{1}$ gehenden Horizontalschnitte, mit $G I I$ den in der Ebene $A S B$ liegenden I) urchmesser des Horizontalschnitts $i$, so ist als Mittellinie in einem Paralleltrapez $G H=v+w$ und als Radius von $i^{\prime} M C_{1}^{\prime}=\frac{v+w}{2}$. Nun ist aber auch $A_{1}^{\prime} B_{1}^{\prime}=v+w$, also $M C_{1}^{\prime}=\begin{gathered}A_{1}^{\prime} B_{1}^{\prime} \\ 2\end{gathered}$, d. h. $M$ ist ein Brennpunkt der Projektion $k_{1}^{\prime}$ der Schnittellipse.

Auf einer beliebigen Mantellinie $S P$ wird der Punkt $P_{1}$ von $k_{1}$ zuerst im Aufriß gefunden als $P_{1}^{\prime \prime}=S^{\prime \prime} I^{\prime \prime} \times e_{2}$, dann im Grundriß auf $M Y$, am genauesten mit Hilfe des Horizontalschnittes der Kegelfläche, der durch $P_{1}^{\prime \prime}$ bestimmt ist. Die 'Tangente in $P_{1}$ an $k_{1}$ ist die Schnittlinie von $E$ mit der Berülırungsebene des Kegels in der Mantellinie SP; sie geht also durch den Schnittpunkt. $T$ ' von $e_{1}$ mit der 'Tangente von $k$ in $I^{\prime}$.

Als wahre Gröbe von $k_{1}$ erhalten wir durch Umlegung in $\Pi_{1}$ eine Ellipse $k_{1}^{0}$ mit den Achsen $A_{1}^{0} B_{1}^{\prime \prime}=A_{1}^{\prime \prime} B_{1}^{\prime \prime}$ und $C_{1}^{00} D_{1}^{0}=C_{1}^{\prime} D_{1}^{\prime}$.

I)ie $A$ bw i ckelung des Kegelmantels ist ein Kreissektor vom Radius $S^{\prime} A=S^{\prime \prime} A^{\prime \prime}$, sein Bogen gleich der Peripherie des Grundkreises $k$. Schneiden wir in der Mantellinie $S B$ auf, so wird der abgewickelte Stumpf symmetrisch in bezug anf $S A$. Die Ausführung gestaltet sich folgendermaßen: Wir teilen die Hälfte von $k$ zwischen $A$ und $B$ in den Punkten 1, 2... in eine Anzahl, z. B. sechs, gleicher Teile und übertragen den Bogen $A 1$ möglichst angenähert auf den Kreis um $S$ mit 
dem Radius $S A^{1}$ ). Vas gefundene Bogenstück wird anf dem Kreist weitergetragen; dann sind die nach den Teilpunkten gehenden Radien die abgewickelten Mantellinien $S 1, S 2 \ldots S B$. Ist $S t^{\prime}$ eine dieser Mantellinien, so erhalten wir den zugehörigen Pnnkt $P_{1}$ der Verwandelten der Schnittellipse $k_{1}$ durch Ermittelung der wahren Länge von $P^{\prime} P_{1}$. Nun entspriclit dem Aufrib $S^{\prime \prime} P^{\prime \prime}$ als wahre Länge von $S P$ dit strecke $S^{\prime \prime} A^{\prime \prime}$; ziehen wir daher durch $P_{1}^{\prime \prime}$ eine Parallele zn $x$, so finden wir auf $S^{\prime \prime} A^{\prime \prime}$ die wahren Längen der Abschnitte $P P_{1}$ und $S P_{1}$. Die 'Tangente der Verwandelten in $P_{1}$ ergibt sich durch Anheften des rechtwinkligen Dreiecks $P_{1} T$, dessen Kathete $P^{T} T$ im Grundric in wahrer Größe erscheint, an die Strecke $P P_{1}$.

Die Wendepunkte der Verwandelten entsprechen denjenigen Punkten $V_{1}$ und $W_{1}$ von $k_{1}$, deren Berührungsebenen auf $\mathrm{E}$ senkrecht stehen (Art. 95). Diese Ebenen gehen durch das Lot von $S$ auf $E$. Ziehen wir aus seinem ersten Spurpunkte $Z$ die Tangente $Z V$ an $k, s 0$ finden wir auf der Mantellinie $S V$ den Punkt $V_{1}$ und damit die 'Tansente von $k_{1}$, die in der Abwickelung zur Inflexionstangente wird.

Bezeichnen wir mit $\rho_{0}$ und $\sigma_{0}$ die Krümmungsradien der Verwandelten in den Scheiteln $A_{1}$ und $B_{1}$, mit $Q$ den Krümmung:radius der Ellipse $k_{1}^{*}$ in $A_{1}^{0}$ und $B_{1}^{\prime \prime}$, mit $\alpha$ und $\beta$ die Winkel bei $A_{1}^{\prime \prime}$ und $B_{1}^{\prime \prime}$ im Dreieck $A_{1}^{\prime \prime} S^{\prime \prime} B_{1}^{\prime \prime}$, so ist nach Art. 95

$$
\boldsymbol{\varrho}_{0}=\frac{\rho}{\cos \psi}, \quad \sigma_{0}=\frac{\rho}{\cos \beta}:
$$

wir erhalten also z. B. $\sigma_{0}$ als Hypotenuse eines rechtwinkligen Dreiecks mit der Kathete $\varrho$ und dem alliegenden Winkel $\beta$. - Unabhängig von der wahren Gröbe $k_{1}^{\prime \prime}$ der Schnittellipse können wir aber die Strecken $\varrho_{0}$ und $\sigma_{0}$ anch leicht direkt ermitteln. Setzen wir nämlich $A_{1} B_{1}=2 a$ und $C_{1} D_{1}=2 b$, so ist bekauntlich $\varrho=\frac{l^{2}}{a}$. Nach Konstruktion ist $O_{1} C_{1}$ die mittlere Proportionale zwischen $G O_{1}=w$ und $O_{1} H=r$, mithin $b^{2}=v w$ und

$$
\varrho=\frac{v w}{a} \text {. }
$$

1) Um den Kreisbogen $\mathfrak{M} \mathfrak{B}$ rom Mittelpunkte $\mathfrak{M}$ und dem Zentriwinkel $\varphi$ auf einen Kreis um ' $\mathfrak{M}^{\prime}$ mit dem Radius $\mathfrak{I}^{\prime} \mathfrak{M} \mathfrak{U}^{\prime}$ zu ïbertragen, zerlegt man $\mathfrak{A} \cdot \mathcal{B}$ in kleine Teile, bei denen man Bogen und Sehne als gleich groß betrachten kann, und überträgt diese Sehnen. Man kann aber auch $\mathfrak{A} \&$ zunächst angenähert rektifizieren. Zu dem Zwecke verlängert man $\mathfrak{A} \mathfrak{M}$ um $\mathfrak{M}(\mathcal{S}=2 . \mathfrak{A} \mathfrak{M}$ und zieht $\mathfrak{A} \mathfrak{D} \perp \mathfrak{X} \mathfrak{M}$ bis zur Geraden (5) $\mathfrak{B}$; dann ist, wenn of den Wert von $60^{\circ}$ nicht wesentlich überschreitet, di Strecke $\mathfrak{A} \mathfrak{D}$ nahezu gleich dem Bogen $\mathfrak{A} \mathfrak{B}$. Diese Konstruktion ist $u m$ so genaver, je kleines $\varphi$ ist, z. B. wird für $\varphi=30^{\circ}$

dagegen

$$
\frac{\mathfrak{A} \mathfrak{D}}{\mathfrak{A} \mathfrak{M}}=\frac{3 \sin \varphi}{2+\cos \varphi}=0,5233 \ldots
$$

$$
\frac{\mathfrak{A} \mathfrak{B}}{\mathfrak{A} \mathscr{H} \mathfrak{C}}=\frac{\pi}{6}=0,5235 \ldots
$$

Macht man nun auf der Verlängerung von $\mathfrak{M}^{\prime} \mathfrak{R}^{\prime}$ die Strecke $\mathfrak{M} \mathfrak{K}^{\prime} \mathfrak{C}^{\prime}=2 . \mathfrak{I}^{\prime} \mathfrak{M} \mathfrak{l}^{\prime}$ und auf einer Senkrechten zu $\mathfrak{H}^{\prime} \mathfrak{M}^{\prime}$ die Strecke $\mathfrak{H}^{\prime} \mathfrak{D}^{\prime}=\mathfrak{I} \mathfrak{D}$, so ist der $z$ wischen $\mathfrak{U}^{\prime}$ und $\mathfrak{E}^{\prime} \mathfrak{D}^{\prime}$ liegende Bogen des zweiten Kreises angenähert $=\mathfrak{A} \mathfrak{Z}$.

Müller, Darstellende Geometrie. 
Nun ergibt sich aus dem Dreieck $A_{1}^{\prime \prime} s^{\prime \prime} B_{1}^{\prime \prime}$, wenn wir den Winkel hei $S^{\prime \prime}$ mit $2 \gamma$ bezeichnen,

oder

$$
\frac{\sin \beta}{\sin 2 \gamma}=\begin{aligned}
& S^{\prime \prime} A_{1}^{\prime \prime} \\
& A_{1}^{\prime \prime} B_{1}^{\prime \prime}
\end{aligned}=\frac{v^{\prime} \cdot \sin \gamma}{2 a},
$$

daher folgt aus Gleichung 2)

$$
{ }_{a}=\begin{aligned}
& \sin \beta \\
& \cos \gamma
\end{aligned}
$$

$$
\varrho=\frac{w \sin \beta}{\cos \gamma}
$$

Ziehen wir demmach $B_{1}^{\prime \prime} J^{\prime \prime} \perp S^{\prime \prime} B^{\prime \prime}$ bis $S^{\prime \prime} U^{\prime \prime}$ und fällen von .J" auf $e_{2}$ ein Lot, das $e_{2}$ in $K^{\prime \prime}$ und $S^{\prime \prime} B^{\prime \prime}$ in $L^{\prime \prime}$ schneidet, so ist der Punkt $K$ ron $A_{1} B_{1}$ der Krümmungsmittelpunkt der Schnittellipse in $B_{1}$, und der Punkt $L$ der Nantellinie $S B$ wird in der Abwickelung zum Krümmungsmittelpunkt der verwandelten Kurve an der entsprechenden Stelle. denn es ist

$$
\begin{aligned}
& B_{1}^{\prime \prime} J^{\prime \prime}=\frac{w}{\cos \gamma^{\prime}}, \\
& B_{1}^{\prime \prime} K^{\prime \prime}=B_{1}^{\prime \prime} J^{\prime \prime} \sin \beta
\end{aligned}
$$

oder nach 3) $=$ o, und

$$
B_{1}^{\prime \prime} L^{\prime \prime}=\frac{B_{1}^{\prime \prime} K^{\prime \prime}}{\cos \beta},
$$

also nach 1) $=\sigma_{0}$.

102. b) Parabolischer Schnitt. In Fig. 101 ist $E$ parallel zur Berührungsebene des Kegels in der Umrißlinie SB, die Schnitt-

Fig. 101.

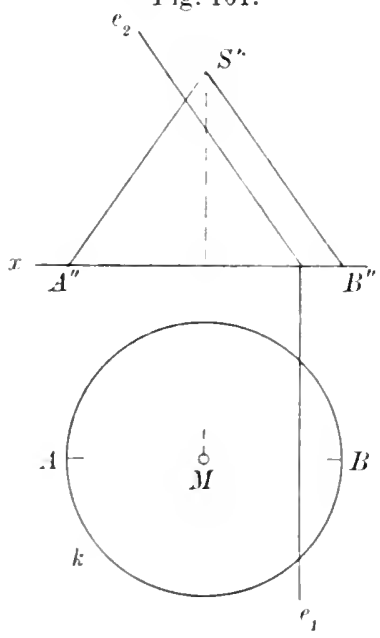
kurve $k_{2}$ also eine Parabel mit dem Scheitel $A_{1}$ und der Achsenrichtung $S B$. Sie geht durch die Schnittpunkte von $e_{1}$ mit $k$; weitere Punkte auf beliebigen Mantellinien werden wie unter a) gefunden. Der Grundrib vou $k_{1}$ ist gleichfalls eine Parabel, und zwar mit dem Scheitel $A_{1}^{\prime}$ und dem Brenupunkt $M$; ihr Krümmungsradius in $A_{1}^{\prime}$ ist folglich, wie in der analytischen Geometrie gezeigt wird, $=2$. $A_{1}^{\prime} M$.

103. c) Hyperbolisclier Schnitt (Fig. 102). Aus dem Aufriß finden wir die Scheitel $A_{1}$ und $B_{1}$, sowie den Mittelpunkt $O_{1}$ der Hyperbel $k_{1}$. Eine Ehene, die durch $S$ || $E$ grelegt wird, schneidet den Kegel in zwei Mantellinien $S(r, S H$, und diese bestimmen die unendlich feruen Punkte von $k_{1}$; die Asymptoten $g_{1}$ und $h_{1}$ der Hyperbel gehen also durch $O_{1} \| S G$ und SII. Als Tangente im unendlich fernen Punkte von $S G$ ist $g_{1}$ die Schnittlinie von $E$ mit der Berührungsebene des Kegels in dieser 
Mantellinie: sie geht alıo durch den Schnithunkt von $e_{1}$ mit der. 'T'angente von $k$ in $G$. - Der Cirundrils von $k_{1}$ ist eine Hyperbel mit den Scheiteln $A_{1}^{\prime}, B_{1}^{\prime}$ und den Asymptoten $g_{1}^{\prime} \| M\left(i, h_{1}^{\prime} \| M H^{1}\right)$.

104. lie Brennpunkte der ebenen schnitte des geraden Kreiskegels. Der Kegel werde erzeugt durch Drehung des in der Zeichenebene $\Pi_{2}$ liegenden gleichschenkligen Dreiecks $A S B$ nm seine Hölenlinie S.11. Wir schneiden ihn mit der Ebene $\mathrm{E} \perp \Pi_{2}$ in einem Kegelschnitt $k_{1}$. In Fig. 103 ist $k_{1}$ eine Ellipse mit den Scheiteln $A_{1}$ und $B_{1}$ auf $S A$ und $S B$. Konstruieren wir die Kreise $v$ und $v_{*}$, die die Geraden $A_{1} B_{1}, S A$, $S B$ der Reihe nach in $F, H, J$ und $F_{*}, H_{*}$ : $J_{*}$ beriuhren, und deren Mittelpunkte $O$ und $O_{*}$ sich auf $S M$ hefinden, so erhalten wir aus ihnen durch Drehung um $S M$ zwei Kugeln $K$ und $K_{*}$; diese berïhren $E$ in den Punkten $F$ nnd $F_{*}$ und den Kegel in zwei anf $S M$ senkrechten Kreisen $u$ und $u_{*}$ mit den Durchmessern $H J$ und $H_{*} J_{*}$. Eine beliebige Mantellinie $S P$ schneide $k_{1}, u, u_{*}$ bzw. in den Punkten $P_{1}, Q, Q_{*}$. Dann sind $F P_{1}$ und $Q P_{1}^{\prime}$ zwei 'Tangenten der Kugel $\mathrm{K}$ aus dem Punkte $P_{1}$, also ist

Ebenso ist

$$
F P_{1}=Q P_{1} \text {. }
$$

folglich

$$
F_{*} P_{1}=Q_{*} P_{1},
$$

$$
F P_{1}+F_{*} P_{1}=Q Q_{*}=H H_{*},
$$

d. h. konstant für alle Punkte von $k_{1}$. Wir schließen daraus, daß $F$ und $F_{*}$ die Bremnpunkte der Ellipse $k_{1}$ sind. Dasselbe läßt sich beweisen, wenn $k_{1}$ eine Parabel oder eine Hyperbel ist, nur sind bei dem parabolischen Schnitt $O_{*}$ und $F_{*}$ unendlich fern. Wir erhalten demnach den Satz: Schneidet

Fig. 102.

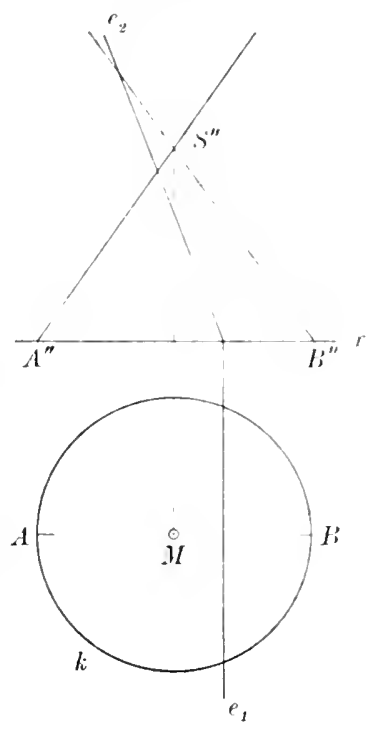

Fig. 103.

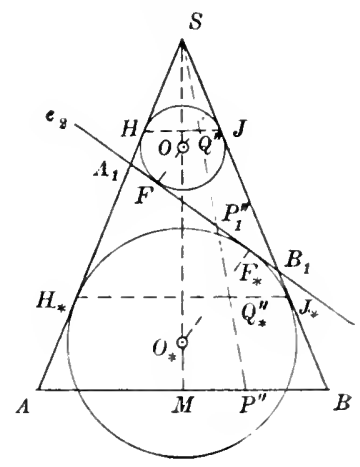
man einen geraden Kreiskegel durch eine Ebene und konstruiert die beiden Kugeln, die den Kegel in einem Kreise und

1) Kennt man ron einer. Hyperbel den Mittelpunkt $\mathcal{E}$, den Scheitel $\mathfrak{A}$ und die Asymprote $g$, so findet man anch sofort den scheitelkrü mung mittelpunkt $\mathfrak{A}$. Sei nümlich $\mathfrak{D} \mathfrak{N}=a$ und die inaginäre Halbachse $=b \sqrt{-1}$ : dann schließt man ans der entsprechenden, für die Eillipse geltenden Formel

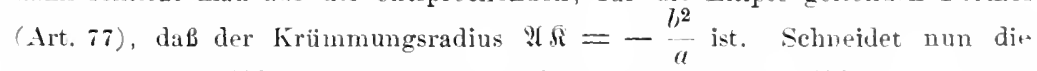
Scheiteltangente $\mathfrak{A}(\mathfrak{5}$ die Assmptote $\mathfrak{g}$ in $(5$, so ist bekanntlich $\mathfrak{A}(5=b$, within ist $\mathscr{H}$ der sichnittpunkt von $E \mathfrak{Q}$ mit dem Lote in 5 zu g. 
die Ebene in einem l’unkte beriilnen, so sind die beiden Berührungspunkte die Brennpunkte des entstehenden Kegel$\therefore$ chll itts.

Sei $G$ der Gegenpunkt von $F$ im kreise $1:$ dann geht die Gerade i $F_{*}$ durch den ïuberen Öhnlichkeitspunkt $s$ von $i$ und $v$. Fine Ebene $\Delta$, die parallel zu $E$ durch den Kegel gelegt wird, schneidet diesen in einem Kegelschnitt $k_{2}$, der $z u k_{1}$ äbnlich ist, und dessen Brempunkte auf den Geraden $S F$ und $S G$ liegen. Nun ist $k_{2}$ der scheinbare Umrib der liugel $\mathrm{K}$ für $S$ als Projektionszentrum und $\Delta$ als Bildebene; d. h.: Der scheinbare Umrib einer in Zentralprojektion dargestellten Kugel ist ein Kegelschnitt, der die Projektionen der Endpunkte des anf der Bildebene senkreehten Kugeldurchmesserszu Brennpunkten hat.

105. Hieraıs ergibt :ich beiläufig die Darstellung der Kugel in schiefer Parallelprojektion. Die projizierenden Strahlen, die die Kugel berühren, bilden einen geraden Kreiszylinder, und dieser schneidet die Zeichenebene $\Pi_{2}$ in der ['mribellipse $u_{s}$, der schiefen Projektion des Hauptkreises $u$, dessen Ebene auf der Projektionsrichtun senkrecht stelit. Das Bild $O_{s}$ des Kugelmittelpunkts $O$ ist der Mittelpunkt ron $u_{s}$. Wählen wir die Projektionsrichtung wie früher (Art.3), -o bildet die Projektion des auf $\Pi_{2}$ senkrecliten Kugeldurchmessers $F G$ mit der Richtung der Projektionsachse $x$ einen Winkel von $30^{\circ}$, und es ist $O_{s} F_{s}=O_{s} G_{s}$ gleich der Hälfte des Kugelradius $r$; damn sind $F_{s}$ und $G_{s}$ die Bremmunkte von $u_{s}$. Jeder Durchmesser von $u$ erscheint im Bilde vergröbert, mit Ausnahme des zu $\Pi_{2}$ parallelen $A B$, der unverändert bleibt. Ziehen wir also $O_{s} \Lambda_{s} \perp O_{s} F_{s}^{\prime}$ und $=r$, so ist $O_{s} A_{s}$ lie kleine Halbachse von $u_{s}$; die grobe Halbachse ist folglich $=F_{s} A_{s}$.

106. Praktische Beispiele zu den ebenen Śchnitten eines yerarlen Kreiskegels. a) Durchdringung eines regelmäbigen sechsseitigen l'rismas mit einem geraden hreiskegel, dessen Achse mit der Achse des Prismas zusammenfällt, und dessen Basiswinkel $30^{\circ}$ beträgt (Schraubenmutter, Fig. 104). Die śeitenflächen des Prismas sind parallel zur Kegelachse und schneiden laher den Kegel in kongruenten Hyperbelbögen $A F, B C \ldots$ Thre scheitel liegen auf dem Kegelkreise, der die Prismaflächen berührt: weitere Punkte erhailt man mittels horizontalen Hilfsebenen. Man findet auch leicht die Asymptoten und die Scheitelkrümmungskreise der zweiten Projektionen der Hyjerbeln.

b) Inrcholringung eines abgestumpften geraden Kreiskegels mit einer regelmäbigen vierseitigen Pyramide (Schornsteinfub, Fig. 105). Lie Jyramidenflachen ASB, BSC.. schneiten den Kegel in vier kongruenten Ellipsenbögen $A_{1} b_{1}, B_{1} C_{1} \ldots$ Hhre Hauptachsen mit den Scheiteln $E_{1}, F_{1} \ldots$ liegen auf den Mittellinien $S E, S F \ldots$ der Seitenflichen. Die vertikale Ebene $1 S C$ schneidet den Kegel in zwei Mantellinien, und diese treffen $S A$ und $S C$ in $A_{1}$ und $C_{1}$. Wreht man die Ebene um die Kegelachse, his sie $\| \Pi_{2}$ wird, so fallen die Ilantellinien mit den zweiten Lmríb des Kegels zonsammen. - 
Zwischenpunkte findet man mit Hilfe von Horizontalschnitten. Man kann auch leicht die Achsen und daraus die Scheitelkrümmungskrei-e der Projektionen der Eillipsenbögen konstruieren.

Fig. 104.
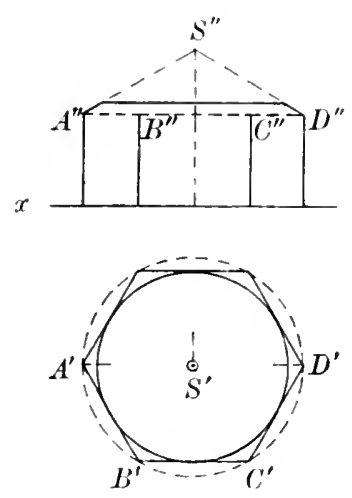

Fig. 105.
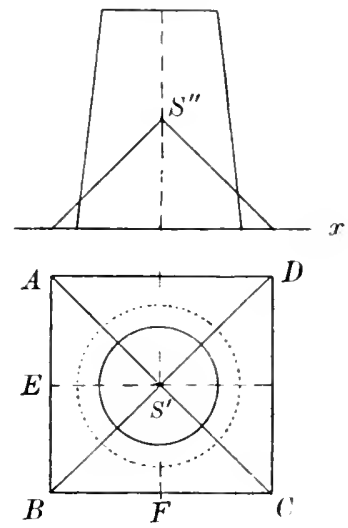

107. Schnitt der Ebene $\mathrm{E}\left(e_{1} e_{2}\right)$ mit einem schiefen Krei- kegel, dessen Grnudkreis $l_{i}$ in $\Pi_{1}$ liegt. Die Schnittkurve $l_{1}$ wird konstruiert, indem man fur eine hinreichende Anzahl von Mantellinien ihre Schnittpunkte mit $E$ ermittelt, wie in Art. 56 unter Benutzung einer $\Pi_{3} \perp e_{1}$. - Die Ebene, die durch die Spitze $S$ und deı Uittelpunkt $M$ von $l_{i} \perp \Pi_{1}$ gelegt wird, teilt den Kegel in zwei symmetrische Hälften und schneidet ihn in seiner längsten und seiner kürzesten Mantellinie $S .1$ bzw. SE. Soll daher ler Kegel, nachdem $k_{1}$ konstruiert ist, anch noch abgrewickelt werden, so empfiehlt es sich, von voruherein den Kreis $k$ von $A$ aus in eine gerade Anzahl gleicher Teile zu teilen und für die nach den Teilpunkten gehenden Mantellinien die Schnittpunkte mit $E$ zu bestimmen. Bei der Abwickelung ersetzt man den Kegel angenähert durch die Pyramide, die jene Mantellinien zu Seitenkanten hat. - Die Punkte, in denen die ersten scheinbaren Umrißlinien den Kreis $k$ berühren, werden in der Abwickelung zu

Fig. 106.

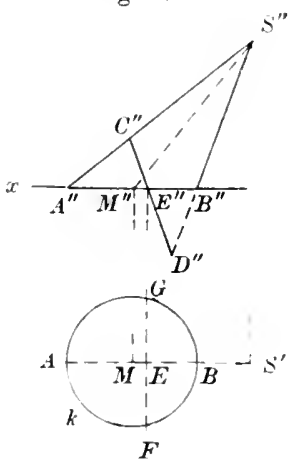
Wendepunkten der Verwandelten von $k$ (Art. 95).

In Fig. 106 ist $S M \| \Pi_{2}$; das Dreieck $A S B$ erseheint alsu in Aufriß in wahrer Größe. Macht man auf $S A$ und $S B$ bzw. die Strecken $S C=S B$ und $S D=S .1$ und bezeichnet mit $E$ den Schnittpunkt von $C D$ mit $A B$, so wird $E C=E B$ und $E D=E A$. Legt man jetzt die Ebene $\mathrm{E}$ durch $C D \perp \Pi_{2}$, so schneidet sie den Kreis $k$ in der durch $E$ gehenden Sehne $F G \perp A B$ und den Kegel in einer Ellipse $k_{1}$. -Diese ist in bezug auf die Gerade $C D$ symmetrisch, hat also $C D$ zur Achse. Man kann nun $E$ um $F G$ drelien, bis $C$ mit $P$ und $D$ mit $A$ 
zusammenfallt; dann hat die Cmlegung $k_{1}^{0}$ von $k_{1}$ mit dem Kiveise $k$ die Achse $A B$ und the Punkte $F$ und $G$ gemein, d. h. $k_{1}^{0}$ deckt sich mit $k$. Die Ebene E schneidet also den Kegel in oinem Kreise. ler dem Kreise $k$ gleich ist. Ter hegel hat demnach zwei Scharen von hreisschniten. von denen die eine $z_{n} \Pi_{1}$, die andere zu $\mathrm{E}$ parallel ist.

Durchdringung zweier hegel-oder Zylinderflächen.

108. Zwei krumme Flächen $A$ und $B$ erzengen als ihre Durehdringung im allgemeinen eine Rammkurve $r$, die man konstruiort, indem man durch $A$ und $B$ eine Schar geeigneter Hilfsfläclen legt und für jede von ihnen ihre Schnittkurven mit $A$ und $B$ ermittelt; die Schnittpunkte beider Kurven sind dann Punkte von $c$. Dabei sind unter .geeigneten" Hilfsflächen solehe zu verstehen, die sowohl A wie B in möglichst einfachen, leicht konstruierbaren Kurven schneiden. In den meisten Fällen wird man Ebenen als Hilfstlächen verwenden, und dann ist jedesmal $z u$ überlegen, was für Ebenen am geeignetsten sind. Handelt es sich z. B. um die Durchlringung einer Kugel mit einem geradeu Kreiskegel, dessen Achse $\perp \Pi_{1}$ ist, so benutzt man horizontale Hilfsebenen, denn diese schneirlen die Kugel und den liegel in Kreisen, die im Grunrlrib in wahrer Größe erscheinen.

109. Eine krumme Fläche heißt von der $m^{\text {ten }}$ Ordnung, wemn ihre Gleichung in rechtwinkligen Koordinaten $\mathfrak{k}, \mathfrak{l}), j$ vom $m^{\text {ten }}$ Grarle ist - oder, was auf dasselbe hinauskommt, wenn sie von jeder nicht in ihr liegenden Geraden in $m$ reellen oder imagrinären l'nukten und

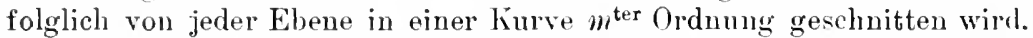
IIan versteht feruer unter ()rdung einer Raumkurve die Anzahl der - reellen oder imagrinären - Punkte, die sie mit einer Ebene gemein hat.

Sind mun die sich durchrlringenden Flächen A und B bzw. von rer $m^{\text {ten }}$ und $n^{\text {ten }}$ Ordnung, so werden sie von einer beliebigen Ebene in zwei Kurven $m^{\text {ter }}$ and $n^{\text {ter }}$ Ordnung gesehnitten, und diese treffen sich bekanntlich in $m n$ - reellen oiler imaginären - Punkten der Durchdringungskurve c. Daraus folgt: \%wei Flächeı $m^{\text {ter }}$ und $n^{\text {ter }}$ Ordunug durchdringen sich in einer Raumkurve m $n^{\text {ter }}$. () $) \cdot(\ln u n g$.

Die Projektion dieser Kurve ist im allgemeinen auch von der m $n^{\text {ten }}$ Ordnung. Denn eine beliebige Gerade der Bildebene hat mit dor Bildkurve $c^{\prime}$ ehenso viel Pnnkte gemein, wie die durch sie gelegte projizierende Ebene mit aler kurve c, d. h. mn Punkte. Auf gewisse Ausnahmen von diesem Satze gehen wir hier noch nicht ain (vgl. 11. a. Art. 117).

110. Cm die [hrehdringungsknrve zweirl Kegelfäehen zu,konstruieren, benutzt man in der Kegel llilfsebenen dur:h die Verbindungrslinie der Spitzen; solche Ebenen scheiden nämlich jede von beiden Flächen in Iantellinien (vgl. Art. 62). 
Sind die Kegelflächen $K$ und $\wedge$ mit den Spitzen $S$ und ' $T$ ' und den Leithurven $k$ und $l$ in den Ebenen $E$ und $\Phi$ gegeben, so ermittle man zunächst die Schnittpunkte $Q$ und $R$ der Geraden $S T$ mit $E$ und $\Phi$, sowie die Schnittlinie $m$ der beiden Ebenen (Fig. 107). Fine Hilfsebene, die durch ST' gelegt wird, schneidet $E$ und Fig. 107.

$\Phi$ in zwei durch $Q$ und $R$ gehenden Gerarlen, die sich auf $m$ treffen, und diese Geraden bestimmen auf $k$ und $l$ die Ausgangspunkte der Mantellinien, welche die Hilfsebene mit den beiden Kegeln gemein hat.

Tritt an die Stelle des einen Kegels ein Zylinder, so legt man die Hilfsebenen durch die Gerado, die durch die Spitze des Kegels parallel zu den

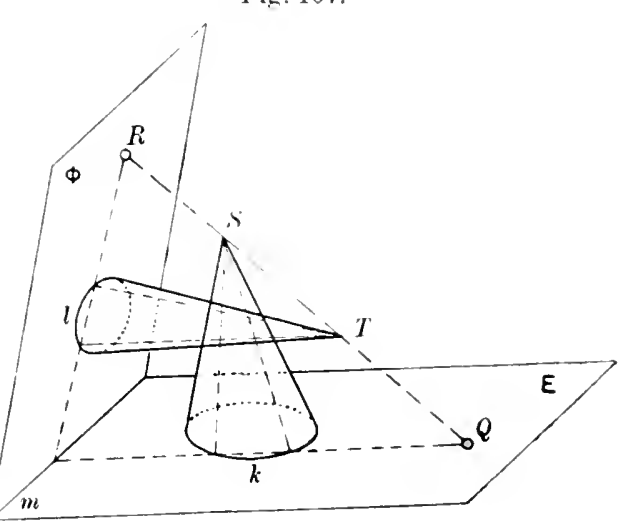
Mantellinien des Zylinders

gezogen wird. Handelt es sich un die Durchdringung zweier Zylinder. so verwendet man Hilfsebenen parallel zu den Mantellinien beider Flächen.

111. Die Konstruktion gestaltet sich besonder's einfach, wenn die Leitkurven $k$ nnd l del beiden Kegelflächen in derselben Ebene, z. B. in $\Pi_{1}$ liegen (Fig. 108). Dann ermitteln wir zuerst den Schnittpunkt $Q$ ron $S T$ mit $\Pi_{1}$; ziehen wir darauf durch $Q$ als Grundrißspur einer durch $S T$ gelegten Hilfsebene die Gerade $u$, die $l_{i}$ in $A$ und $B$, sowie $l$ in $C$ und $D$ schneidet, so bestimmen die Mantellinien $S A, S B$ und $T C, T D$ vier Punkte der Durchdringungskurve $c$.

Trehen wir die Gerade $u$ in $\Pi_{1} \mathrm{um} Q$, so erreicht sie eine Grenzlage $\%$, in der sie $l$ in einem Punkte $G$ berührt und $k$ in zwei Punkten $E$ und $F^{\prime}$ schneidet. Die durch $v$ bestimmte Hilfsebene berührt den Kegel $\wedge$ in $T G$. lie Mantellinien $S E$ und $S F$ sind also Tangenten von $\Lambda$ und folglich der Durchdringungskurvein ihren Schnittpunkten mit $T G$. Das Entsprechende gilt ron der aus $Q$ an $k$ gelegten Tangente $w$, die mit $l$ die Pnnkte $I I$ und.$J$ gemein hat. Lann begrenzen auf $k$ die Punkte $E$ und $F$, auf $l$ die Punkte $H$ und $f$ einen Bogen, dessen Mantellinien den anderen Kegel nicht treffen; im rorliegenden Falle schneiden daher die beiden Kegel einander gegenseitig an, und ihre Durchdringungskurve besteht aus einem einzigen geschlossenen Zuge. Eine vollständige Durchbohrung des Kegels $K$ und eine zweiteilige Durchdringungsknrve würde entstehen, wenn man von $Q$ an $l \mathrm{zwei}$ Tangenten ziehen könnte, die $k$ schneiden.

Die scheinbaren Umrißlinien der beiden Kegelflächen sind Doppeltangenten der Projektion der Durchdringungskurve, soweit sie überhanpt Kurvenpunkte enthalten (Art. 82). 
Bej der Ausfübrung ler Konstruktion bestimmen wir zuerst die ausgezeichneten Punkte von $c$ a uf solchen Mantellinien, die die Kurve berühren, sowie die l'mule anf den Umrißlinien der beiden liegel und legen dann nach Bedürfnis weitere Hilfsebenen in die noch vorhandenen Lücken. l)ie Reihenfolge, in der wir die erhaltenen Punkte verbinden, ergibt sich wie in Art. 12 durch gleichzeitiges Unfahren ler beiden I.eitkurven.

Ein Punkt der Projelition von $c$ ist sichtbar, wenn die beiden durch ihn gehenden Mantelliuien in dieser Projektion sichtbar sind.

Die 'Tangente in einem Punkteder Dnrchdringungskurvit jst die Schnittinieder Berülrungsebeneuder beiden Flächen

Fig. 108.

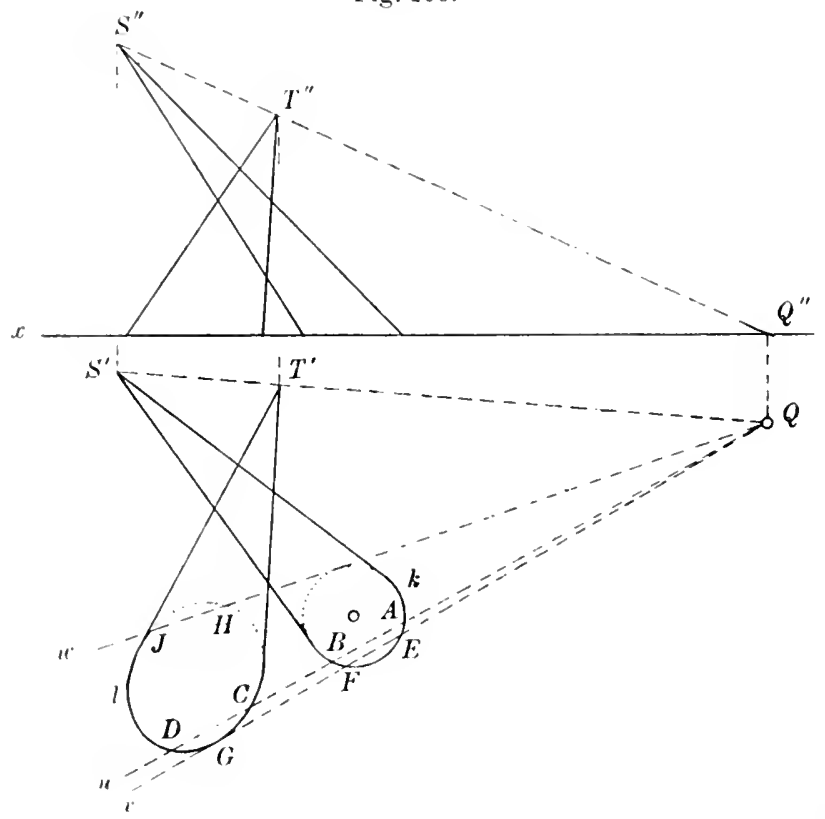

in diesem Punkte. Un hiernach z. B. im Schnittpunkte der Mantellinien $S A$ und $T D$ die 'Tangente $t$ von $c$ zu konstruieren, ziehen wir als Grundribspuren der zugehörigen Berührungsebenen die Tangenter von $k$ in $A$ und von $l$ in $D$; diese schneiden sich im ersten Spurpunkte von $t$.

112. Singuläre Punkte in Bilde der Durchdringung:kurve. In Fig. 108 hat der Grundrif $c^{\prime}$ der Kurve $c$ zwei Doppelpunkte. Ein solcher Ioppelpunkt entsteht in Bilde einer Raumkurve immer dann, wenn ein projizierender Strahl die Originalkurve zweimal chneidet. - Rüeken die beiden Sehnittpunkte einander unendlich nahe, berührt also der projizierende Strabl die Originalkurve in oinem Punkte I', so rieht sich in Bilde die zum Woppelpunkt gehörige Schleif in den Punkt $I^{\prime}$ zusammen, d. h. die Bildkurve hat in $I^{\prime}$ einen R Rick kelirpunkt. 
Jeder Punkt der Originalkurve, dessen Schmiegungsebene eine projizierende Ebene ist, liefert im Bilde einen Wendepunkt. Dann entsprechen nämlich den drei unendlich nahen Punkten, welche die Schmiegungsebene mit der Originalkurve gemein hat, auf der Bildkurve drei unendlich nahe Punkte in einer Geraden. Dies gilt natürlich nicht mehr, wenn die Schniegungsebene eine gerade Anzahl unendlich naher Punkte der Raumkurve enthält (Art. 88).

113. Unendlich ferne Punkte der Durchdringungskurvo *utstehen als Schnittpunkte paralleler Mantellinien der beiden Kegelflächen. Um solche Mantellinienpaare zu ermitteln, denken wir uns den einen der beiden Kegel - in Fig. 108 den Kegel K, weil dessen Leitkurve $k$ ein Kreis ist - parallel zu sich verschoben, bis seine Spitze $S$ mit der des anderen, $T$, zusammenfällt. Der verschobene Ḱegel schneidet die $\Pi_{1}$ in einer Kurve $k_{1}$; dann sind $k_{1}$ und $k$ zwei ähnliche

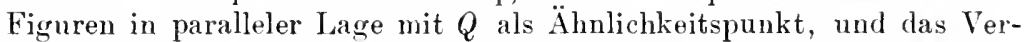
hältnis entsprechender Strecken ist $=Q T: Q S$. Ist $k$ ein Kreis mit dem Mittelpunkte $M$, so liegt der Mittelpunkt $M_{1}$ von $l_{1}$ anf $(\mathcal{M}$, , und zwar so, daß $T M_{1} \| S M$ ist.

Bezeichnen wir mit $P_{1}$ einen sichnittpunkt von $k_{1}$ und $l$, mit $I^{\prime}$ den entsprechenden Punkt von $k$ auf der Geraden $Q P_{1}$, so sind di Iantellinien $S P$ und $T P_{1}$ parallel; ihre Berührungsebenen schneiden sich also in der'Tangente des zugehörigen unendlich fernen Punktes, d. h. in einer Asymptote der Durchdringungskurve. Diese ist $\| T P_{1}$ und seht durch den Schnittpunkt der Tangenten an $l$ in $L^{\prime}$ und an $l$ in $P_{1}$. - Da in Fig. $108 l_{1}$ und $l$ einander nicht schneiden, so hat dim Kurve $c$ keine (reellen) unendlicl fernen Punkte.

114. In Fig. 109, die nur den Grundrib darstellt, sind die Kegel so gewählt, daß die Leitkurven $k$ und $l$ eine gemeinsame Tangente $v$ aus $Q$ mit den Berührungspunkten $F$ und $G$ besitzen. Dann berühren sich die Kegelflächen im Schnittpunkte $P$ von $S F$ und $T G$. und $v$ ist die Grundribspur der gemeinschaftlichen Berührungsebene. Nähert sich die durch $S T$ gelegte Hilfsebene dieser Grenzlage, so fallen vier Punkte derDurchdringungskurve $c$ gleichzeitig in $P$ zusammen; $P$ ist daher ein Doppelpunkt von $c$ : Das erkennt man auch noch in folgender Weise: Jede durch $P$ Fig. 109.

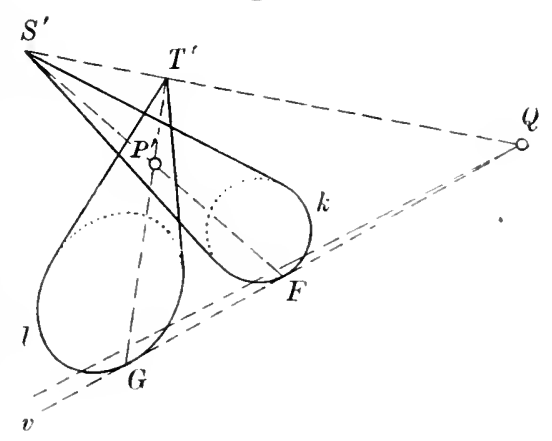
gehende Ebene schneidet die beiden Kegelflächen in zwei Kurven, die sich in $P$ berühren; in jeder solchen Ebene zählt also $P$ für zwei Schnittpunkte mit $c$. Diese Schlubweise gilt allgemein, nicht nur für Kegelflachen; wir erhalten daber den Satz: Berühren sich zwei Flächen in einem Punkte, so ist er ein Doppelpunkt ihrer Durchdringungskurve. 
115. Durchdringung zweier Zylinderflächen, deren Leitkurven $k$ und $l$ in $\Pi_{1}$ liegen (Fig. 110). Nach Art. 109 schmeiden wir die Zylinder mit Hilfsebenen, die zu den Iantellinien beider Flächen

Fig. 110 .

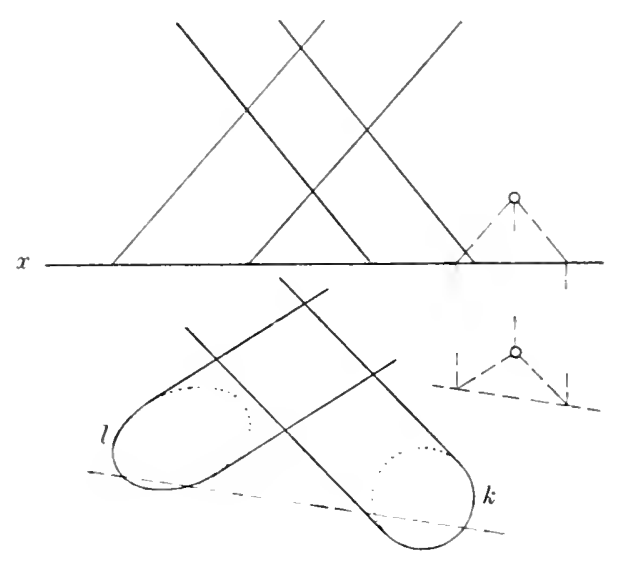

parallel sind. Um die Richtung der Grundribspuren solcher Ebenen zu bestimmen, ziehen wir durch irgend einen Punkt im Raume zu den Mantellinien die Parallelen $g$ und $h$ und ermitteln von der Ebene $g h$ die Grundri@spur $G_{1} H_{2}$.

116. Durchdringung zweier geraden Kreiszylinder mit \%ueinander windschiefen Achsen $a \perp \Pi_{1}$ und $b \| \Pi_{2}$ und den

Fig. 111.
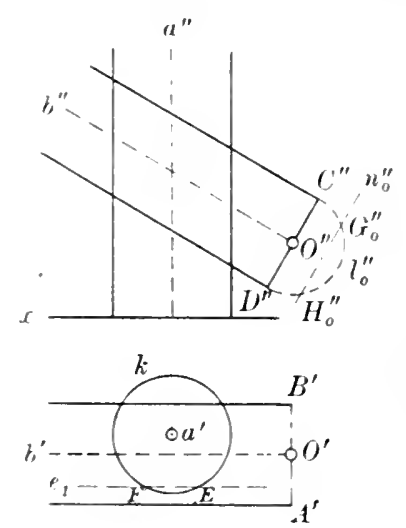
Grundkreisen $k$ in $\Pi_{1}$ und $l$ mit dem Jittelpunkt $O$ und den Durchmessern $A B \| \Pi_{1}$ und $C D \| \Pi_{2}$ (Fig. 111). Die Durchdringungskurve $c$ fällt im Grundriß mit einem Teil von $k$ zusammen, es handelt sich also uur um die Konstruktion von $c^{\prime \prime}$. Die vorhin benutzten Hilfsebenen sind gegenwärtiq $\| \Pi_{2}$ : wir bezeichnen die durch $b$ gelegte mit $B$, irgend eine andere, deren Grundribspur $e_{1}$ sei, mit $E$. Diese schneidet die Ebene von $l$ in einer Geraden $n \| C D$ und die Zylinder in Nantellinien durch die Punkte $E, F$ $=e_{1} \times k$ und $G, H=n \times 7$. Uin $G^{\prime \prime}$, $I^{\prime \prime}$ zu ermitteln, drehen wir den vorderen Hallokreis $7 \mathrm{um} C D$ in die Lage $l_{0} \| \Pi_{2}$ : damn ist Abstand $n_{0}^{\prime \prime}, C^{\prime \prime} I^{\prime \prime}=$ Abstand $e_{1} b^{\prime} \mathrm{usw}$.

Wir konstruieren noch die 'T angente in irgend einem Punkte ron $c$, z. B. im Schnittpunkte $P$ der durch $E$ und $G$ gehenden Mantellinien. \% dem \%wecke ermitteln wir von den Berührungsebenen der \%ylinder in PE und $P G$ ihre Schnittlinien mit B, $u \| a$ und $v \| b$. 
Diese gehen durch die Schnittpunkte der 'Tangenten in $F$, an $k$ und in $G$ an $l$ (d. h. in $G_{0}$ an $l_{0}$ ) bzw. mit $b^{\prime}$ und $C^{\prime} D$. Der Schnitpunkt von $u^{\prime \prime}$ und $c^{\prime \prime}$ bestimmt den Aufriß der gesucliten 'Tangente.

Anstatt den Kreis $l$ um $C D$ in B nm\%ulegen, kann man ihn durch I) rehung um $A B$ in die Lage $7^{0} \| \Pi_{1}$ bringen. Kommt hierdurch $G$ nach $G^{0}$, so ist $G^{0^{\prime}}$ der Schnittpunkt von $l^{0^{\prime}}$ mit $e_{1}$, und dann ist $O^{\prime \prime} G^{\prime \prime}=$ Abstand $G^{\prime} 0^{\prime}, A^{\prime} B^{\prime}$.

117. Durchdringung zweier geraden Kreiszylinder mit sich schneidenden Achsen. Die Zylinder sind in Fig. 112 nur im Grundrib gezeichnet, und zwar liegen ihre Achsen $a$ und $b$ in $\Pi_{1}$; rlie Grundkreise $k$ und $l$ sind gegeben durch ihre in $\Pi_{1}$ befindlichen Durchmesser $A B \perp$ " und $C D \perp b$. Die durch die Endpunkte gehenteu Mantellinien bestimmen sofort vier Punkte 1, 2. 3, 4 der Durchdringungskurve $c$, die in bezug auf $\Pi_{1}$ symmetrisch ist. Lm weitere Punkte von $c^{\prime} z u$ erhalten, schneiden wir die Zylinder oberhalb $\Pi_{1}$ mit horizontalen Hilfsebenen. Eine dieser Ebenen, im

Fig. 112.

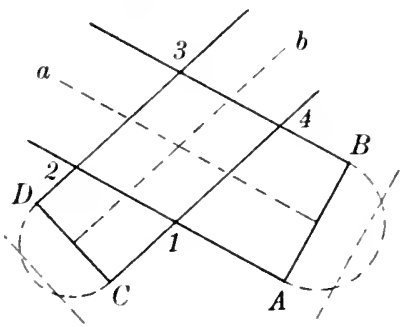

Abstande $h$ von $\Pi_{1}$. trifft die Ebenen von $l_{i}$ und $l$ in den Geraden $m \| A B$ und $n \| C D$; dabei ist Abstand $m, A B=$ Abstand $n, C D=h$. Die Schnittpunkte von $m$ und $n$ bzw. mit $l$ und $l$ finden wir durch Cinlegung rer über $A B$ und $C D$ stehenden Halbkreise in $\Pi_{1}$, usw.

Nach Art. 109 ist die Schnittlinie zweier Kegel- oder Zylinderflächen zweiter Orlnung eine Raumkurve vierter Ordnung; ihre Projektionen sind also im allgemeinen ebene Kurven von derselben Ordnung. Gegenwärtig ist aber jerler Punkt von $c^{\prime}$ der Grundriß zweier Punkte von $c$. Legen wir daher durch irgend zwei Punkte $P^{\prime}$ und $Q^{\prime}$ von $c^{\prime}$ eine Ebene $\perp \Pi_{1}$, so schneidet diese die Kurve $c$ in vier. Punkten, rie paarweise denselben Grundrif $P^{\prime}$ oder $Q^{\prime}$ besitzen. Die Gerade $I^{\prime}\left(Q^{\prime}\right.$ hat folglich mit $r^{\prime}$ keinen dritten Punkt gemein, und der Grundrib der Durchdringungskurre ist also ronder zweiten Ordnung. Er besteht aus zwei doppelt zählenden Bögen 12 und 34 einer Hyperbel, die $a$ und $b$ zu konjugierten I)urchmessern hat, deun jede von beiden Geraden halbiert die zur andern parallelen Sehnen.

Betrachten wir nur die oberhalb $\Pi_{1}$ liegenden Halbzylinder, so stellt die Figur die Durchdringung zweier Tonnengewölbe dar.

Haben die beiden Zyliuder gleiche Radien, so berühren sie sich in zwei Punkten $V$ und $W$ des $i m$ Schnittpunkte von $a$ und $b$ zu $\Pi_{1}$ errichteten Lotes. Dann sind die Ellipsen, welche die Strecke $V W$ und je eine Diagonale des Rhombus $1234 \mathrm{zu}$ Achsen haben, den beideu Zylindern gemeinsam: die Durchdringungskurve zerfällt daher in diese beiden Ellipsen, also in zwei ebene Kurven. Dieser Fall liegt z. B. beim Kreuzgewölbe und beim Klostergewölbe ror.

118. In Fig. 113 haben wir die beiden Zylinder durch $\mathrm{zwei}$ gerade Kreiskegel ersetzt, deren Achsen $S M$ und $T N$ in $\Pi_{1}$ liegen. Im hier die Durchdringungskurse $c$ zu konstruieren, bestimmen wir 
zunähst die Schnittpunkte $Q$ und $R$ der Geraden $S T$ bzw. mit den in $\Pi_{1}$ befindlichen Durchmessern $A B$ und $C D$ der Grundkreise $k$ und 7 , sowie den Schnittpunt $O$ von $A B$ und $C D$; durch ihn geht $\perp \Pi_{1}$ dit Schnittlinie $i$ der Ebenen von $k$ und $l$. Durch Umlegnng dieser Ebenen in $\Pi_{1}$ kommt $i$ in die Lagen $i_{1} \perp O Q$ nud $i_{2} \perp O R$; gleichzeitig gelangen lie oberen Halhkreise $k$ und $l$ nach $k_{0}$ und $l_{0}$.

Eine durch S'T gelegte Hilfsebene sehneidet die Ebenen ron $h$ und $l$ in zwei durch $Q$ und $R$ gehenden Geraden, die sich in einem

Fig. 113.

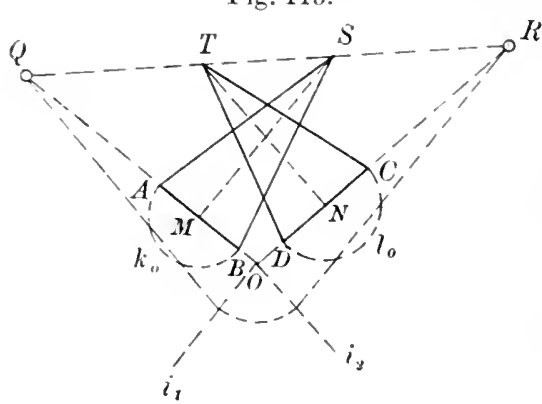

Puukte $f$ von $i$ treffen, in der Unlegung in $Q J_{1}$ und $R \cdot J_{2}$ : (labei ist $O J_{1}=O J_{2}$. Die Geraden $Q J_{1}$ und $R J_{2}$ bestimmen auf $l_{0}$ und $l_{0}$ die Ausgangspunkte der Mantellinien, in denen die Hilfsebene die beiden Kegel schneidet.

Die 'T a ngente in einem Punkte von $c$ ergibt sich wieder als Scbnittlinie der Berülnrungsebenen der beiden Kegel im betrachteten Punkte. Von jeder dieser Ebenen bestimme man zuerst ihre Schnittlinie mit der zugehörigen Grundkreisebene und daraus die Grundribspur.

Man beweist wie bei der vorhergehenden Aufgabe, dab der Grundrib $c^{\prime}$ von $c$ aus Teilen einer Kurve zweiter Ordnung besteht.

Anmerkung. Die Anwendung horizontaler Hilfsebenen, wie in Art. 117, hätte hier keinen Sinn, denn solche Ebenen schneiden die Kegel nach Hyperbeln.

119. Durchdringung eines geraden Kreiskegels mit einem geraden hreiszylinder, wenn die Achsen beider Flächen zueinander windschief sind (Fig. 114). Der Grundkreis $k$ des Kegels liegt in $\Pi_{1}$; die Achse $a$ des Zylinders ist $\| \Pi_{2}$, sein Grundkreis l befindet sich also in einer $z \Pi_{2}$ senkrechten Ebene $\Phi\left(f_{1} f_{2}\right)$. Wir ziehen dureh die Spitze $S$ des Kegels eine Parallele zn $a$, bestimmen ihre ichnittpunkte $Q$ und $I$ bzw. mit $\Pi_{1}$ und $\Phi$ und klappen die Ebene $\Phi$ in $\Pi_{1}$ nm: dadurch gelangen $l$ und $R$ naeh $l_{0}$ und $I_{0}$. Hierauf legen wir als erste Spur einer durch $S Q$ gehenden Hilfsebene durch (? eine Gerade, die $k$ in $E$ und $F$ und $f_{1}$ in $J$ schnoidet; dann ist $J R_{\text {, }}$ die umgeklappte Schnittlinio der Hilfsebene mit $\Phi$. In ihren Schnittpunkten $G_{0}$ und $J_{0}$ mit $l_{0}$ bestimmen wir auf $f_{2}$ den Anfrib und ziehen lurch $E, F$ und $G_{n}, H_{0}$ sowie durch $E^{\prime \prime}, F^{\prime \prime}$ und $G^{\prime}, I^{\prime \prime}$ die Projektionen der Mantellinien, welche die Hilfsebene mit den beiden Fläehen semein hat.

In Fig. 115 ist die Achse a des Zylinders $\| x$. Konstruieren wir hier den Seitenrif auf eine $\Pi_{3} \perp a$, die in $\Pi_{1}$ umgelegt wird, so fallt die dritte Projektion der Durchdringungskurve $c$ mit einem 'Teil von $l^{\prime \prime}$ zusammen; un also die Schnittpunkte einer beliebigen Mantellinie des Kegels mit dem $/ y$ linder zu bestimmen, brauchen wir diese Mantellinie mur im Seitenri屯 zu zeichnen. So ermitteln wir insbesondere die Punkte 
von $c$ auf den zweiten und dritten Umrißlinien des Kegels, sowie anf den Mantellinien, die den Zylinder berühren. - Die Konstruktions-

Fig. 114.

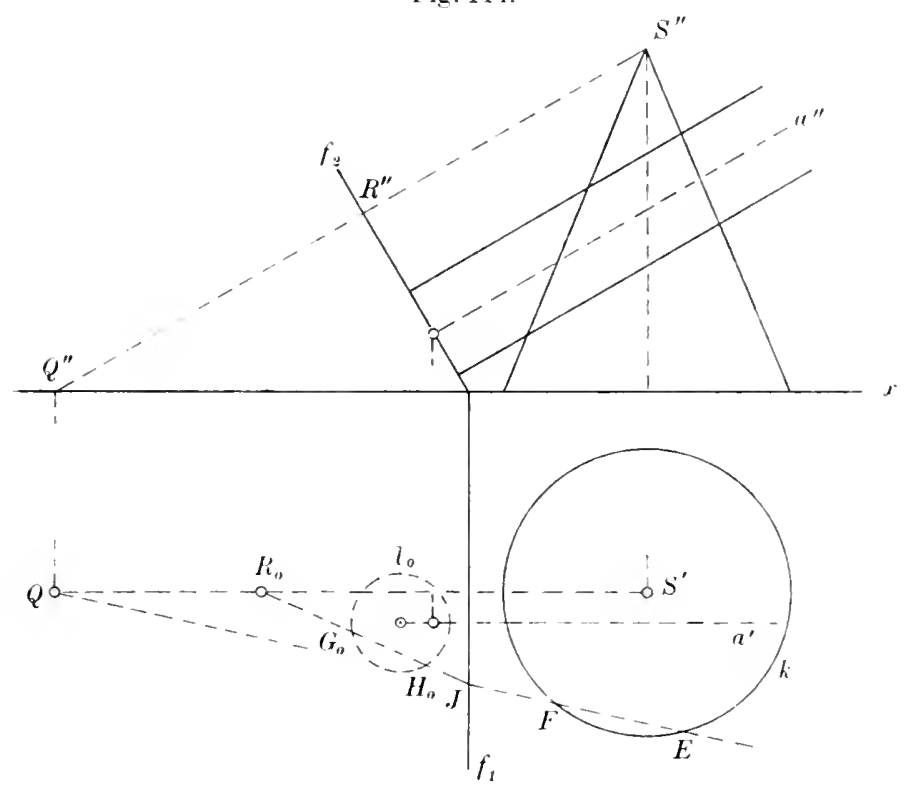

linien ändel'n sich nicht, wenn wir, wie bei der vorhergehenden Figur. durch die spitze des Kegels eine Parallele zu a ziehen und durch diese Gerade eine Schar von Hilfsebenen legen.

Im vorliegenden Falle können wir aber, abweichend von der allgemeinen Regel, auch horizon tale Hilfsebenen benutzen. Solche Ebenen schneiden nämlich len Kegel in Kreisen und den Zylinder in Paaren von Mantellinien, die aus dem Seitenril. leicht bestimmt werden können. Dieses Verfahren eignet sich besonders zur Ermittelung der Punkte von $c$ auf den ersten und zweiten Lmriblinien des Zylinders.

120. Hilfsebenen senkrecht zur hegelachse erweisen

Fig. 115 .

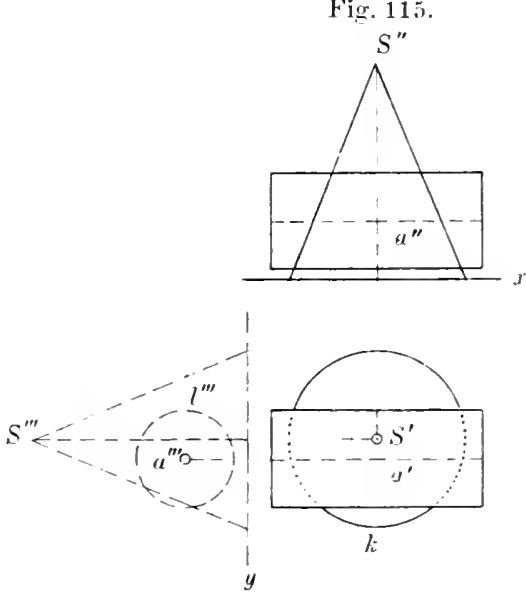
sich gleichfalls als vorteilhaft bei der in Fig. 116 dargestellten Durchdringung, wie sie bei Maschinenteilen häufig vorkommt. Hier ist die Achse $a$ des geraden Kreiszylinder $\perp \Pi_{1}$ : die Achse $b$ des geraden 
abgestumpften Kireiskegels schneidet "rechtwinklig nud ist anberdem $\Pi_{2}$. ller Grundriß der Durchdringungskurve $c$ fält in den Grundkreis des Zylinders. Daraus funden wir zunachst die P'nnlite von a" anf solchen Mantellinien des Kegels, die zu einer der Projektionsebenen

Fig. 116 .
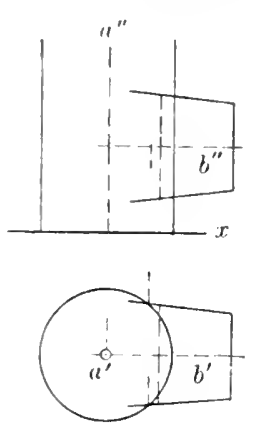
parallel sind. Eine Hilfsebene $\perp b$ schneidet den Kegel in einem Kreise. Die anf ihm liegenden Punkte von $c$ sind im Grundrib bekannt, wir erhalten sie daher im Aufriß wie in Art. 81 mittels eines Seitenrisses. Die Kinre $c$ ist in bezug anf die Ebene a $b$ symmetrisch, ilu Aufriß besteht daher aus zwei Teilen einer Hyperbel (vgl. Art. 117 ).

121. In Fig. 117 ist die Achse $b$ des geraden Kreisliegels gegen $\Pi_{1}$ geneigt. aber wie vorher $\| \Pi_{2}$, und sie schneidet die Achse $a$ des aufrecht stehenden geraden Kreiszylinders (zylindrische Kanne mit konischer Schnauze). Tom Kegel ist die Spitze $S$ und der Grundkreis $l$ durch einen Durchmesser $C D \| \Pi_{2}$ gegeben. Der Grundrib der Durchrlringungskurve $c$ fällt wieder in den Grundkreis $k$ des Zylinders, wir erhalten daher $c^{\prime \prime}$, indem wir eine Reihe von Kegelmantellinien in

.. Fig. 117 .
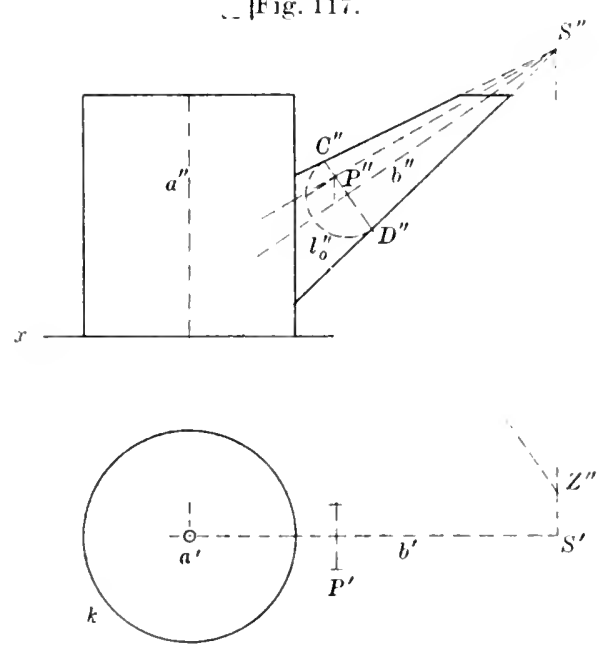

(irund- und Aufrib zeichnen und die Schnittpunkte ihrer ersten Projektionen mit $k$ ermitteln. Ist $S^{\prime \prime} P^{\prime \prime}$ der Aufrip einer beliebigen Mantellinie, lie $l$ in $P$ schneiclet, so finden wir den Punkt $I^{\prime \prime}$, indem wir den vorleren Halbkreis $l \mathrm{um} C D$ in die lage $l_{0} \| \Pi_{2}$ drehen: dadurch kommt $I^{\prime}$ nach $I_{0}^{\prime}\left(P^{\prime \prime} I_{0}^{\prime \prime} \perp C^{\prime \prime} I^{\prime \prime}\right)$, und dann ist Abstand $P^{\prime} b^{\prime}=P^{\prime \prime} P_{0}^{\prime \prime}$.

Mit $S^{\prime} P^{\prime}$ deckt sich der (irundrib einer zweiten liegelmantellinie $S Q$, leren Aufrif man in folgender Weise ermittelt: Die erste pro- 
jizierende Ebene von $S l$ schneilet die Ebene ron $l$ in der Verbindungslinie von $P$ mit dem Schnittpunkte $Z$ von $S S^{\prime}$ und $C D$, und die Gerade $P Z$ trifft $l$ zum zweitenmal in $Q ; Q_{0}^{\prime \prime}$ ist also der zweite Schnittpunkt von $I_{0}^{\prime \prime} Z^{\prime \prime}$ mit $l_{0}^{\prime \prime}$. Die auf $S P^{\prime}$ und $S Q$ liegenden Punkte von $c$ haben denselben Grundrif. - Man bestimme insbesondere die ersten l'mrißlinien des Kegels mittels der Tangente aus $Z^{\prime \prime}$ an $l_{0}^{\prime \prime}$, sowie 'lie zugehörigen Punkte von $c$.

Die Kurve $c^{\prime \prime}$ ist abermals ein Hyperbelbogen.

122. Durchdringung eines halben geraden Kreiszylinders, dessen Mantellinien $\| x$ sind, mit einem schiefen Kreiskegel, dessen Leitkurve - der Halbkreis $l-\| \Pi_{2}$ liegt (Tonnengewïlbemit konischer Stichkappe, Fig. 118). Die Durchlringungskurve $c$ fällt im Seitenriß in den Grundkreis $k$ des Zylinders, ihr Aufriß ergibt sich daher, indem man genügend riele Mantellinien des Kegels in beiden Projektionen zeichnet. Man kann aber anch Hilfsebenen

Fig. 118.

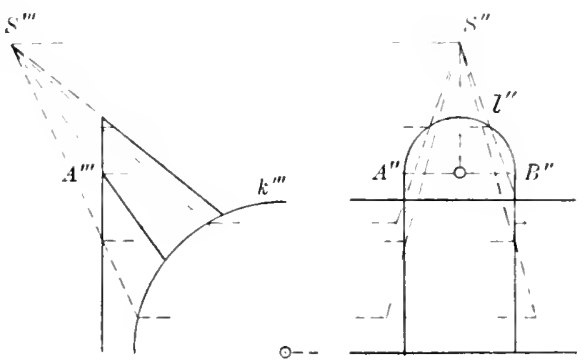
$\Pi_{2}$ benutzen, die man zuerst im Seitenrjo angibt; sie schneiden den Zylinder in Mantellinien und den Kegel in Halbkreisen, deren Aufriß sofort konstruiert werden kann.

Als Fortsetzung der Kurve $c$ ermittle man noch die elliptischen Scbnitte des Zylinders mit vertikalen Ebenen durch die letzten Kegelmantellinien $S A$ und $S B$, am einfachsten mit Hilfe von GGaden, welche die Punkte der durch $A$ und $B$ gehenden Vertikalen mit der Spitze $S$ verbinden.

123. Sonderfälle bei der Durchdringung zweier Kegelflächen zweiter Ordnung. a) Wenn zwei Kegelflächen zweiter Ordnung (mit verschiedenen Spitzen) eine IIantellinie gemein haben, so durchdringen siesich überdiesineiner Raumkurvedritter Ordnung. Fig. 119 zeigt zwei solche Kegel, deren Leitkurven $k$ und $l$ in der Zeichenebene liegen, in Parallelprojektion auf diese Ebene. Der früher mit $Q$ bezeichnete Spurpunkt der Verbindungslinie der Spitzen $S$ und $T$ liegt jetzt sowohl auf $l$, als auch auf 7 . Die Konstruktion der Durchdringungskurve bleibt dieselbe wie in Art. 111. Die Kurve geht durch die beiden Spitzen. -- Da

Fig. 119 .

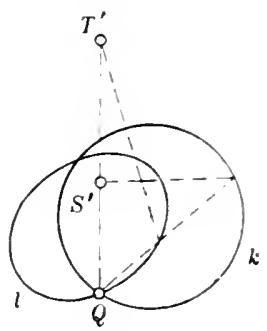
es Raumkurven erster oder zweiter Ordnung nicht gibt ${ }^{1}$ ), so haben die Raumkurven dritter Ordnnng für die Geometrie des Raumes eine ähnliche Bedeutung, wio die Kegelschnitte für die Ordnung.

1) Zwei windschiefe Geraden bilden eine meigentliche Paumkurre zweiter 
Geometrie der Ehene; man bezeichnet sie deshalb anch zuweilen als kubische legelschnitte.

b) Berïhren sich die beiden Kegelflächen in der gemeinsamen In antellinie, so ist der Rest der lourchelingungskurve ein Kegelschnitt. Dieser Fall tritt in Fig. 119 ein, wenn die Kegelschnitte $l i$ und $l$ sich in l'unkte $Q$ berühren.

$\mathrm{Zu}$ einem Beispie] einel solchen Durchdringung, das auch praktisch nicht ohne Bedeutung ist, gelangt man in folgender IVeise (Fig. 120). Durch I)rehung des in der Zeichenebene liegenden rechtwinkligen Drei-

Fig. 1:0.

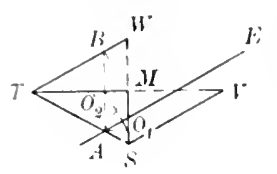
ecks $S M T$ b\%w, un seine katheten $M S$ und $M T$ entstehen zwei gerade Kreiskegel $I$ und $I I$, die sich längs der gemeinsamen Mantellinie $S T$ berühren; sie schneiden die Zeichenebene noch in den parallelen cievaden $S T$ und $T$ ' $I T$. Fallen wir von $H$ auf $S T$ das Lot $M .1$ und ziehen durch seinen Fulpunkt die Gerade $A E \| S V$, so schneidet

die Ebene $\mathrm{E}$, die durch $A \mathrm{E}$ senkrecht zur Zeichenebene gelegt wird, die beiden Kegelflächen in zwei Parabeln $p_{1}$ und $p_{2}$ mit dem Scheitel $A$ und der Achse $A E$. Um ihre Brenmpunkte $F_{1}$ und $F_{2}$ zu bestimmen: benutzen wir die beiden Kugeln, welche dit Kegel in je einem Kreise und auferdem die Ebene $E$ beriuhren (Art. 104). Ihre llittelpunkte $O_{1}$ und $O_{2}$ liegen anf $S H$ und $T M$, und zwar ist $A O_{1} \| T H$ und $A O_{2} \| S M$. Dann sind $F_{1}$ und $F_{2}$ die Fubpunkte der von $O_{1}$ und $O_{2}$ auf $A E$ gefällten Lote. Bezeichnen wir aber nit $B$ den Schnittpunkt von $A O_{2}$ und $T H$, so ist $O_{1} O_{2} \| M B$, also $\perp T W$ und folglich auch $\perp A E$, d. h. die Brennpunkte $F_{1}$ und $F_{2}$ sind identisch mit dem Schnittpunkte $F$ von $O_{1} O_{2}$ und $A E$. Demnach fallen auch die Parabeln $p_{1}$ und $p_{2}$ zul-ammen, d. h. die Kegelflächen durchdringen sicl in der senk-

Fig. 121.

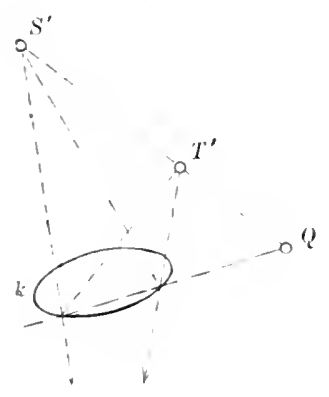
recht zur Zeichenfläche liegenden Parabel mit dem Scheitel A und dem Brenupunte $F$. Denken wir uns daher (len Kegel $I$ als Hohlform ausgebildet und den Kegel $I I$ mit Masse erfüllt, so darf seine Mantellinie nicht gröber angenommen werden als die Strecke $T A$, wenn er in der Hohlform Platz finden soll.

c) Enthatten die beiden Kegelflächen denselben Kegelschnitt $k$, aber keine gemeinsane Mantellinie, so haben sie anberdem noch einen zweiten Kegelschnitt miteinander gemein (Fig. 121). Dieser Kegelschnitt geht durch die lierihrungspunkte der 'Tangenten aus dem spurpunkte $Q$ von $S T$ an $k$ : in beiden Punkten haben die Kegelflächen gemeinsane Berührungsebenen. Umgekehrt gilt der Satz: Wenn zwei Kegelflachen zweiter Ordnung sich in zwei Punkten berühren, so zerfält ihre lourchdringungskurve in zwei Kegelschuitte (vgl. Art. 117, S(chluli).

d) Ilaben zwei Keqelflichen zweiter On'dnung gemeinsame Spitze, so zerfallt ihre lourchdringungskurvo in vier (reelle oder inaginäre) Mantellinien. 
Schlagschatten a uf Kegrel- und Zylinderflächen, sowie auf krummen Flächen überhaupt.

124. Die Grenzlinie des Schlagschattens, den eine krumme Fläche A bei Parallelbeleuchtung von einer anderen Fläche $B$ empfängt, ist ein Teil der Durchdringungskurve von A mit dem Lichtstrahlenzylinder, der die Eigenschattengrenze bzw. den Rand von B zur Leitkurve hat. Wir konstruiereu sie entweder nach dem direkten oder nach dem indirekten Terfahren (Art. 69 und 70).

I. Beim direkten Verfahren ermitteln wir von den die Fläche B streifenden Lichtstrahlen unter Anwendung geeigneter Hilfsebenen die Punkte, in denen sie die Fläche A zum ersten Male schneiden. - Legen wir durch beide Flächen eine Reihe von Ebenen parallel zur ersten projizierenden Ebene des gegebenen Lichtstrahles l, so schneidet jede von ihnen $A$ und $B$ bzw. in zwei Kurven $a$ und $b$. Dann treffen die Tangenten, die wir $\| l^{\prime \prime}$ an $b^{\prime \prime}$ ziehen, die Kurve $a^{\prime \prime}$ in Punkten der zweiten Projektion der gesuchten Schlagschattengrenze. Ilieses Verfahren liefert zugleich - im allgemeinen wenig genan - in den Berührungspunkten der zu $l^{\prime \prime}$ parallelen Tangenten von $a^{\prime \prime}$ und $b^{\prime \prime}$ eine Reibe von Punkten der Eigenschattengrenzen beider Flächen.

II. Um nach dem indirekten Verfahren den Schlagschatten vou $B$ auf $A$ zu finden, konstruieren wir von $B$ und von einer Schar passend gewählter Kurven von $A$ den Schlagschatten auf eine der Projektionsebenen, z. B. auf $\Pi_{1}$. Bezeichnet $i$ eine Kurve der Schar, $i_{h}$ ihren Schatten auf $\Pi_{1}, P_{h}$ einen Schnittpunkt von $i_{h}$ mit der Schlagschattengrenze von B, so bestimmt der durch $P_{h}$ rückwärts gezogene Lichtstrahl auf $i$ einen Punkt $P$, und dieser gehört zur Grenze des auf A geworfenen Schlagschattens, falls er sich im beleuchteten Teile der. Fläche A befindet. - Ist A eine Kegel-oder Zylinderfläche, so ersetzen wir jene Kurvenschar naturgemäß durch Mantellinien des beleuchteten Flächenteils. Bei der Konstruktion des Schlagschatten: anf einer Kugel benutzen wir als Hilfskurven eine Schar horizontaler Kreise usw.

125. Trifft die Grenzlinie des auf die Fläche A fallenden Schlagschattensdie Eigenschattengrenze der Fläche in einem Punkte $Q$, so ist die Tangente der schlagschattengrenze in $Q$ den Lichtstrahlen parallel. Im allgemeinen wird nämlich der Lichtstrahl, der in einem Punkte der Schlagschattengrenze die Fläche A trifft, in diese eindringen und sie mindestens noch einmal schneiden. Aber der durch $Q$ gehende Lichtstrahl berübrt $A$ an der betrachteten Stelle, hat also mit der Fläche und folglich mit der Durchdringungskurve, von der die Schlagschattengrenze einen Teil bildet, zwei zusammenfallende Punkte gemein.

126. Durchdringen sich die Flächen $A$ und $B$ in der Kurve $c$, und trifft diese die Eigenschattengrenze $s$ von $B$ im Punkte $P$, so berührt die Grenzlinie $s_{*}$ des ron B a f A geworfenen Schlagschattens die Kurve $c$ in $P$. Denn $s_{*}$ ist ein Teil der Durchdringungskurve von A mit dem Lichtstrahlenzylinder, 
Jer B in s beribht. folylich ergibt sich die Tangente von $s_{*}$ in $l$ ' als schnittlinie der zugehörigen Berührunısebenen an A und den Zylinder. Die Zylinderberührungsebene ist aber identisch mit der Berührungsebene von B in $P$, und diese schneidet die Berihrungsebene von A in der 'Jangente von $c$.

127. Schattenkonstruktion bei einem aufrecht stehenden geraden Kreis\%ylinder mit quadratischer Deckplatte (Fig. 122).

Fig. 12:.
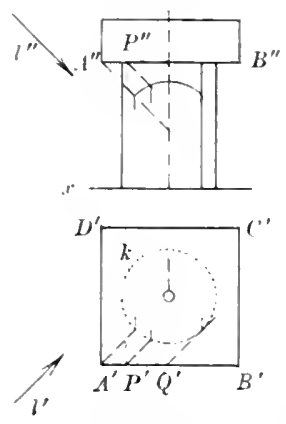
Der Schlagschatten der zu $x$ parallelen Kante $A B$ auf den Zylinder ist ein Ellipsenlogen, nämlich der sichnitt des Zylinders mit der durch $A B$ gelegten Lichtstrahlenebene $\mathbf{E}$. Jian erhält ihn nach dem direkten Verfahren, indem man durch eine Reihe von Punkten A, I'... der Kante Lichtstrahlen zieht und deren Schnittpunkte $A_{*}, I_{*}{ }_{*} \ldots$ mit der Zylinderfïche ermittelt. Der Bogen hat seinen Endpunkt $Q_{*}$ auf der Eigenschattengrenze des Zylinders und wird in diesem Punkte rom Lichtstrahl $Q Q_{*}$ berührt (Art. 125).

Für Licht in der Richtung der Würfeldiagonale ist die Ebene $E$ parallel zur Halbierungsebene $H_{1}$ des von der $+\Pi_{1}$ mit der $+\Pi_{2}$ gebildeten Winkels. Nach Art. 8 sind aber Grund- und Aufriß jeder in $\mathrm{H}_{1}$ liegenden Figur in bezug auf $x$ symmetrisch; für jede Figur in $\mathrm{E}$ sind also ihre beiden Projektionen ungleichsinnig kongruent, mithin

Fig. 123.

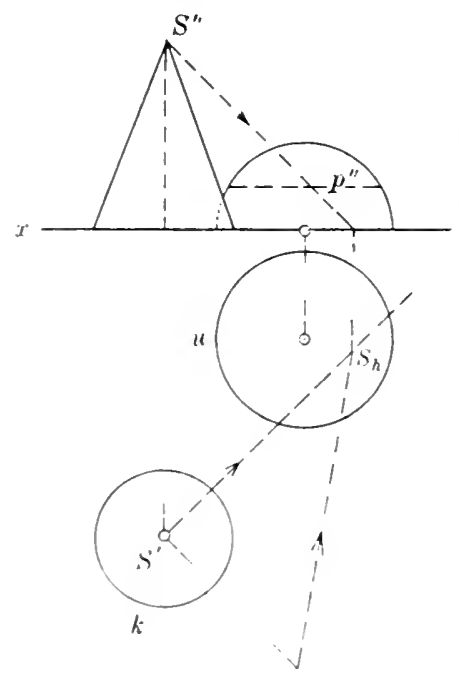

ergibt sich als Aufrib des Ellipsenbogens $A_{*} Q_{*}$ ein Kreisbogen von demselben Radius, wie der Grundkreis $k$ des Zylinders: sein Mittelpunkt liegt im Aufrib der Zylinderachse und hat ron $A^{\prime \prime} B^{\prime \prime}$ denselben Abstand, wie der Jittelpunkt von $k$ von der Geraden $A^{\prime} B^{\prime}$.

128. Schlagschatten eines geraden Kreiskegels a u eine $\mathrm{Halb}$ kugel, wenn die Grundkreise $k$ und $u$ der beiden Flächen sich in $\Pi_{1}$ befinden (Fig. 123). Wir konstruieren zunächst von der Spitze $S$ des Kegels den Schatten $S_{h}$ auf $\Pi_{1}$; dann sind die Tangenten aus $S_{h}$ an $k$ die Grenzen des Kegelschattens auf $\Pi_{1}$, und ihre Berülırungrspunkte $E$ und $F$ bestimmen die Eigrenschattengrenzen des Kegels. Die Berührungsebenen des Kegels längs SE' und SF schneiden die Ilalbkugrel in zwei Kreisbögen, den Grenzen des auf ihr erzeugten Schlagschattens: diese beginnen in den schnittpunkten $G$ und $I I$ von $u$ bzw. mit $S_{h} E$ und $S_{h} F$ und treffen sich im schatten $S_{*}$ von $S$, falls der Lichtstrahl $S S_{h}$ die Halbkugel schneidet. Der lunkt $S_{*}$ wird nach 
dem direkten Verfahren gefunden: Die erste projizierende Ebene von $S S_{h}$ schneidet die Halbkugel in einem Halbkreise $i$; durch Cimlegung in $\Pi_{1}$ ergibt sich $S_{* 0}$ als Schnittpunkt von $S_{0} S_{h}$ mit $i_{0}$ (Art.85). Uu von den Kreisbögen $S_{*} G$ und $S_{*} H$ eine Reihe ron Zwischenpunkten zu ermitteln, benutzen wir das indirekte Verfahren. Zu den Zwecke konstruieren wir von einem horizontalen Kreise $p$ der Halbkugel den Schatten $p_{h}$ auf $\Pi_{1}$, indem wir von seinem Mittelpunkte $O$ den Schatten $O_{h}$ bestimmen. Dann erhalten wir aus den Schnittpunkten von $p_{h}$ mit den Geraden $S_{h} E$ und $S_{h} F$ durch Zurückprojizieren in der Lichtrichtung auf $p$ zwei Punkte der gesuchten Kreisbögen.

Die Konstruktion der Eigenschattengrenze $s$ der Halbkugel gestaltet sich hier etwas einfacher als in Art. 87. Schneiden wir nämlich die Halbkugel mit der ersten projizierenden Ebene des durch den Mittelpunkt $M$ gehenden Lichtstrahls in einem Halbkreise $w$ und ziehen au diesen in fler Lichtrichtung eine 'Tangente, so ist der Grundrib C' des Berührungspunktes (' bekanntlich ein Endpunkt der kleinen Achse der Ellipse $s^{\prime}$. Legen wir nun die projizierende Ebene in die $\Pi_{1}$ um, so fällt $w$ auf $u$; ziehen wir daher an $u$ die Tangente $C_{0} C_{h} \| S_{0} S_{h}$, so ist $C_{0} C^{\prime} \| S_{0} S^{\prime}$. Die 'Tangente liefert auf $M C^{\prime \prime}$ sofort den Endpunkt $C_{h}$ der großen Achse der Schlagschattengrenze $s_{k}$.

129. Konstruktion des Schlagschattens, den der ebone Rand eines hohlen geraden Kreiskegels auf die Innenseite der Kegelfläche wirft. In Fig. 124 befindet sich der Kreis $k$, der den Rand des Kegels bildet, in einer horizontalen Ebene E; die Spitze $S$ des Kegels liegt unterhalh $E$ in $\Pi_{1}$, und der durch $S$ gehende Lichtstrahl schneidet $\mathrm{E}$ in $I$. Ziehen wir aus $L$ an $K$ 'Tangenten mit den Berührungspunkten $E$ und $F$, so sind $S E$ und $S F$ die Eigenschattengrenzen des Kegels, und zwar ist der zwischen ihnen liegende, dem Punkte $L$ zugewendete 'Teil der Fläche anßen beleuchtet und innen im Eigenschatten.

Der durch $l i$ gelegte Lichtstrablenzylinder schneidet nach Art. $123 \mathrm{c}$ den Kegel in einem zweiten Kegelschnitt $k_{*}$, d. h. in einer Ellipse, da auf einem Kreiszylinder weder Parabeln, noch Hyperbeln liegen. Die beiden Flächen berühren einander in $E$ und $F$, folglich geht $l_{*}$ durch diese

Fig. 124.

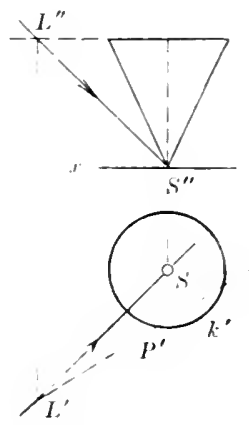

Punkte. Der schattenwerfende Bogen des Kroises $k$ reicht also von $E$ bis $F$, und sein Schlagschatten ist der durch dieselben Punkte begrenzte Ellipsenbogen $i_{*}$.

In gleicher Weise ergibt sich allgemein der. Satz: Der Schlagschatten eines ebenen Randes einer Fláche zweiter Ordnung auf diese Fläche ist ein Kegelschnitt, der von den Schnittpunkten der Randkurve mit der Eigenschattengrenze a usgeht.

Um den Ellipsenbogen $k_{*}$ zu konstruieren, ziehen wir durch irgend einen Punkt $P$ des schattenwerfenden Kreisbogens den Lichtstrahl $P$ und ermitteln seinen zweiten S'chnittpunkt ${ }^{\prime}{ }^{\prime}$ mit der Kecrelfläche: Die 
Ebene durch $p$ und $S$ schneidet $E$ in der Geraden $L_{1} P$ '. 'Trifft diese den Kreis $k$ znm zweiten Male in ?. so hat die Ebene $p S$ mit den hegel die Mantellinie $S Q$ gemein, und dam ist $P_{*}$ der Schnittpunkt von $p$ mit $S Q$. - Die Tangente ron $l_{*}$ in $P_{*}$ ist die Schnittlinie der Ebene ron $k_{*}$ mit der Berührungsebene dos Kegels in $I_{*}$. Diese Ebenen schneiden $\mathrm{E}$ in $E F$ und in der Tangente von $k$ in $Q$; die gesuchte Tangente geht also durch den Schnittpunkt beider Geraden. Durch denselben Punkt geht ancl die Tangente von $f$ in I' als Schnittlinie ron $E$ mit der Berührungsebene des Lichtstrahlenzylinders in der Mantellinie $p$.

Der Ellipsenbogen $l_{*}$ ist symmetrisch in bezug auf die erste projizierende Ebene der Geraden $I S S$; der Mittelpunkt $G$ des Kreisbogens $E F$ erzengt daher als seinen Schatten $G_{*}$ den Scheitel von $k_{*}$. Wir konstruieren den Punkt $G_{*}$ zuerst im Aufriß, oder durch Drehung jener ersten projizierenden Ebene um die Kegelachse, bis sie $\| \Pi_{2}$ wird.

130. Konstruktion des Inuenschattens bei einem hohlen geraden Kreiszylinder. der durch die Kreise $k$ und $k_{1}$ mit den Jittelpunkten $M$ und $M_{1}$ begrenzt wird. Die Lichtrichtung ist durch den Strahl $l$ gegeben.

a) Die Zylinderachse $M M_{1}$ ist $\perp \Pi_{1}$ (Fig. 125). Wir bestimmen zunächst die Eigenschattengrenzen $E E_{1}$ und $F F_{\mathrm{I}}$ uittels

Fig. 125.

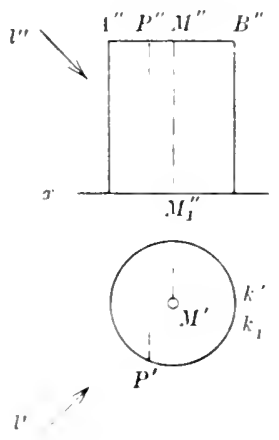

Fig. 126.

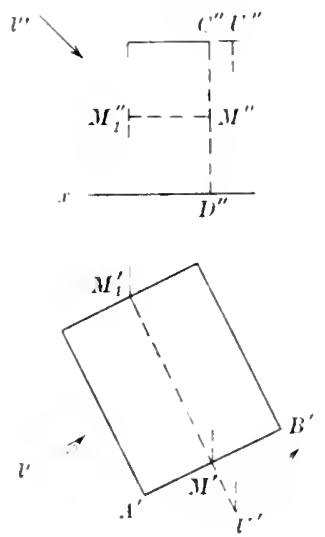

Tangenten ||$l^{\prime}$ an $k^{\prime}$. I) Schlagschatten. Ien der obere Rand $l:$ auf die Innenseite der Zylinderfläche wirft, ist nach Art. 129 eine Hall,allipse $k_{*}$ mit $E F$ als Durchmesser. Von einem beliebigen P'nukte $I^{\prime}$ von $k$ erbalten wir sofort den Schatten $P_{*}$, indem wir $P^{\prime} P_{*}^{\prime} \| l^{\prime}$ bis $k^{\prime}$ ziehen. So ermitteln wir insbesondere den auf die Comriblinie $B B_{1}$ fallenden Schatten. - Die Ellipse $k_{*}$ ist perspektiv affin $z \wedge k$ : koustruieren wir daher zum Endpunkte $C_{r}$ des anf $E F$ senkrechten Durch-

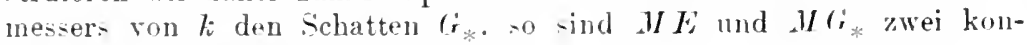
jugierte Durchmesier von $k_{\star x}$. 
b) Wie Achse $M_{1} M_{1}$ ist $\| \Pi_{1}$, aber gegen $\Pi_{2}$ beliebig geneigt (Fig. 126). Projizieren wir $k$ und $l$ auf eine $\Pi_{3} \perp M_{1} M_{1}$, so finden wir die Eigenschattengrenze und den Inneuschatten des Zvlinders mit Hilfe des Seitenrisses in derselben Weise wie unter a).

Wir können aber die Aufgabe auch ohne Seitenriß lösen: Sei E die Ebene des Kreises $k, A B$ der horizontale, $C D$ der vertikale Durchmesser. Ziehen wir lurch irgend einen Punkt von li, z. B. durch $C$, den Lichtstrahl $c$, so erhalten wir seinen zweiten Schnittpunkt $C_{*}$ mit dem Zylinder, indem wir durch $c$ und die Mantellinie $C C_{1}$ eine Ebene $\Gamma$ legen und die Mantellinie bestimmen, in der $\Gamma$ den Zylinder nochmals schneidet. Zu dem Zwecke ziehen wir durch einen beliebigen Punkt $U$ von $C C_{1}$ den Lichtstrahl $U V$ bis zur Ebene $\mathrm{E}$, al:o $U^{\prime \prime} V^{\prime} \| l^{\prime}$, bis $A^{\prime} B^{\prime}$, dann ist $C V$ die Schnittlinie von $\Gamma$ mit $\mathrm{E}$ - oder der Schatten von $C C_{1}$ auf $E$. Trifft $C V$ den Kreis $k$ in $W$, so hat die Ebene $\Gamma$ mit rem Zslinder die Mantellinie $W W_{1}$ gemein, und diese schneidet $c$ in $C_{*}$. Für irgend einen anderen Punkt $P$ von $k$ ist die Ebene durch den zugehörigen Lichtstrahl $p$ und die Mantellinie $P J_{1} \| \Gamma$ : um also im Aufriß den Schatten $I_{*}^{\prime}$ von $P$ zu ermitteln, ziehen wir einfach $P^{\prime \prime} Q^{\prime \prime} \| C^{\prime \prime} \mid V^{\prime \prime}$ bis $k^{\prime \prime}$ und $Q^{\prime \prime} P_{*}^{\prime \prime} \| x$ bis $\mu^{\prime \prime}$. Auf diese Weise ergibt sich u. a. der Berührungspunkt vou $k_{*}^{\prime \prime}$ mit der Cmriblinie $D^{\prime \prime} D_{1}^{\prime \prime}$ - - Die Tangenten an $k^{\prime \prime} \| C^{\prime \prime} W^{\prime \prime}$ liefern die Ansgangspunkte $E^{\prime \prime}$ und $F^{\prime \prime}$ der zweiten Projektionen ler Eigenshattengrenzen des Zylinders, wie auch der Halbellipse $l_{*}^{\prime \prime}$.

131. Innenschatten bei einer hohlen Halbkugel. In Fig.127 ist der Randkreis $u \| \Pi_{1}$ : I bezeichnet den durch den Mittelpunkt $M$ gelegten Lichtstrahl. Die Ebene der Eigenschattenurenze geht bekanntlich durch $M \perp l$ und schneidet $u$ in dem auf $l^{\prime}$ senkrechten Turchmesser $A B$. Der Schlagschatten $u_{*}$, den die Hälfte des Kreises $u$ in die Hohlkugel wirft, ist also ein Kegelschnittsbogen zwischen den Endpunkten $A$ und $B$, d. h. ein Halbkreis mit $A B$ als Inrchmesser, sein Fig. 127 Grundrib eine Halbellipse mit der sroben Achse $1^{\prime} B^{\prime}$. Die erste projizierende Ebene ron 7 schneidet die Halbkugel in einem Halbkreis $w$ und den schattenwerfenden Rand in $E$. Der durch $E$ gehende Lichtstrall trifft $w$ nochmals in $E_{*}$; dann ist $E_{*}^{\prime}$ der Endpunkt der kleinen Hallarhse von $u_{*}^{\circ}$. Wir konstruieren ihn, indem wir die erste projizierencle Ebene von $l$ nm den vertikalen Kugeldurchmesser drehen, bis sie $\| \Pi_{2}$ wirl, oder auch mittels einer $\Pi_{3}$. die z 11 jener projizierenden Ehene parallel ist ( $\mathrm{rgl}$. Alt. 87).

132. Innenschatten bei einer. Mauernische, die ducheinen lualben geraden Kreiszylinder und eine ihn überleckende Viertelkugel begrenzt wird (Fig. 128). Ter zu $\Pi_{2}$ parallele Randkreis $k$ erzeugt in der Halbkugrel einen kreisförmigen Schatten $l_{*}$, der unter Vertauschung von Grund-und Aufriß - ebenso konstruiert wird, wie bei der vorhergehenden Aufgabe. Er beginnt also im Punkte $A$ ron $k$; dessen Tangente zu $l^{\prime \prime}$ parallel ist, und projiziert sich im Aufrib 
al- Ellipsenbogen mit der großen Malbachse $\boldsymbol{M}^{\prime \prime} \boldsymbol{A}^{\prime \prime}$. Weitere Punkte dieses Bogens werden anch in folgender Weise gefunden: Eine Ebene E $\| \Pi_{2}$ mit der Grundribspur $e_{1}$ schneidet die Viertelkugel in einem

Fig. 128.

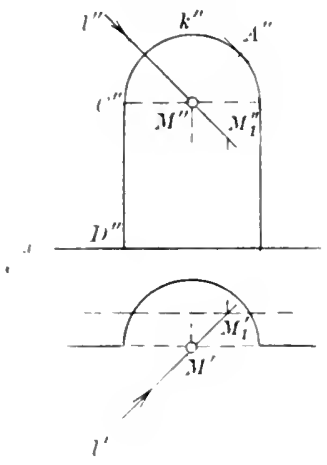
Halbkreise $i$. Der Schatten von $k$ auf $E$ ist ein zu $k$ kongruenter Halbkreis $k_{1}$, sein Mittelpunkt $M_{1}$ der Schatten vou $M$. Dann geht $l_{*}$ durch den Schnittpunkt von $k_{1}$ mit $i$.

Die Schattengrenze $k_{*}$ reicht bis zu ihrem Schuittpunkte $B_{*}$ mit dem horizontalen Halbkreise $u$, in dem sich Viertelkugel und Halbzylindel berühren. Der durch $B_{*}$ geheude Lichtstralıl schneidet $k$ in einem Punkte $B$. Dann fällt der Schatten des Bogens $B C$ von $k$ in den Hohlzylinder und ist ein Teil einer Raumkurve vierter Ordnung. Wir konstruieren ihn, indem wir von einigeu Punkten des Bogens die durch sie gehenden Lichtstrahlen in Grundund Aufriß zeichnen und ihre Schnittpunkte mit dem Zylinder bestimmen. Die Kurve $B_{*} C_{*}$ berührt in $B_{*}$ den Kreisbogen $A_{*} B_{*}$. weil Kugel und Zylinder in $B_{*}$ dieselbe Berührungswhene haben. und sie berührt in $C_{*}$ den Schatten der Mantellinie CD.

\section{Umdrehungsflächen.}

Eigenschaften der Umdrehungsflächen. Berührungsebenen und ebene Schuitte.

133. Eine Lmdrehungsfläche (Drehfläche, Rotationsfläche) entsteht, wenn eine nnveränderliche Kurve sich um eine feste Gerade dreht, bis sie in ihre urspringliche Lage \%ur ückkommt. Die Kurve heißt die Erzeugende, die feste Gerade die Achse der Umdrehungsfläche. Jeder Punkt der Erzeugenden beschreibt bei der Drehung einen Parallelkreis der Fliche, dessen Ehene auf der Arhse senkrecht steht, und dessen Mittelpunkt anf der Arhse liegt.

Jede anf der Fläche liegende Kurve, die jeden Parallelkreis schneidet, kann als Erzengende der Fläche betrachtet werden.

Jele durch die Achse gelegte Ehene schneidet die Fläche in einer lleridiankurve. Alle Meridiankurven sind kongruent und symmetrisch in bezug auf die Achse, wie sich sofort ergiht. wenn man eine Merirliankurve als Erzengende auffaßt.

134. Sei a die Achse, $m$ die Meridiankurve einer UmdrehungsAlche, I' ein beliebiger J'unkt von $m, I ' S$ ' die zugehörige 'Tangente (Fig. 129). Durch $l$ geht ein Parallelkreis $p$; sein Mittelpunkt $Q$ ist der Fubpunkt rles Lotes von I' anf $a$. Die 'Tangenten in $I^{\prime}$ an $m$ und $p$ bestimmen die Berübrungsebene $T$ der Fläche in $P$. Da die Parallelkreistangente auf ler Meridianebene senkrecht steht, so gilt dasselbe von der Ebene T, d. h.: Die Berührungsebene einer Undrehungsflache ist senkrecht auf der Meridianebene des Berührungs- 
punktes. Deshalb fält die Flächennorinale in $P$ zusammen mit der Meridiannormalen $I^{\prime} O$.

Beschreiben wir um den Schnittpunkt $O$ von $P O$ und $a$ einen hreis mit dem Radius $O P$ und drehen die ganze so erhaltene Figur um $a$, so erzeugt PS einen Kegel und der Kreis eine Kugel, Fig. 129. und dann ergibt sich der Satz: Jede Umdrehungsfläche wird längs eines Parallelkreises von einem Cindrehungskegel und von einer Kugel berührt. deren Mittelpunkte auf der Achse liegen. - Sie wird längs jeder Meridiankurve von einem Zylinder berïhrt, dessen Mantellinien auf der Meridianebene senkrecht stehen.

135. Darstellung +iner Umdrehungsfläche,

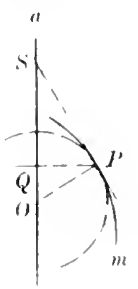
deren Achse $a$ auf $\Pi_{1}$ senkrecht stelit (Fig. 130). In der zu $\Pi_{2}$ parallelen Meridianebene $M_{0}$ sei die Meridiankurve $m_{0}$ gegeben; sie bildet den zweiten wahren Umrib der Fläche nnd soll deshalb als Umrils- oder Hauptmeridian bezeichnet werden. Zum zweiten Umriß gehören außerdem die Parallelkreise derjenigen Punkte ron $m_{0}$, deren Tangenten auf $a$ senkrecht stehen. Der erste Umrib besteht aus den Parallelkreisen solcher Punkte von $m_{0}$. deren Tangenten $\| \mid a$ sind.

Ist der Punkt $P$ der Umdrehungsfläche durch seinen Grundriß $P^{\prime}$ gegeben, so finden wir seinen Aufrib mit Hilfe des Parallelkreises $p$, der durch $p$ geht, und dessen Grundrib $p^{\prime}$ bekannt ist.

Die Berührungsebene $\mathrm{T}$ der Fläche in $P$ ist nach dem Vorigen durch Parallelkreis- und Meridiantangente von $P$ bestimmt. Drehen wir die durch $P$ gehende Meridiankurve um $a$, bis sie mit $m_{0}$ zusammenfällt, so gelangt $P$ nach $P_{0}$, und die zugehörige Meridiantangente wird zur Tangente von $m_{0}$ in $P_{0}$. Diese möge $a$ in $S$ und $\Pi_{1}$ in $R_{0}$ schneiden: machen wir dann auf $a^{\prime} P^{\prime}$ die Strecke $a^{\prime} R=\mathrm{Ab}$ stand $R_{0}^{\prime \prime} a^{\prime \prime}$, so ist $R$ der erste Spurpunkt der Meridiantangente in $P$. Die erste Spur $t_{\mathrm{I}}$ von $T$ geht durch $R$ parallel zur Parallelkreistangente in $P$. also $\perp a^{\prime} R$. 'Trifft $t_{1}$ die durch $a^{\prime}$ zn $x$ gezogene Parallele in $T$, so ist $S T$ die in der Ebene $M_{0}$ liegende Hauptlinie von T, mithin $t_{2} \| S^{\prime \prime} T^{\prime \prime}$. - Die Gerade $S T$ kann auch ohne vorhergehende Bestimmung von $t_{1}$ unmittelbar konstruiert werden. Ziehen wir nämlich $P_{0}^{\prime \prime} O^{\prime \prime} \perp P_{0}^{\prime \prime} S^{\prime \prime}$ bis $a^{\prime \prime}$, so ist $P^{\prime \prime} O^{\prime \prime}$ der Aufriß der Flächennormale in $P$, also $S^{\prime \prime} T^{\prime \prime} \perp P^{\prime \prime} O^{\prime \prime}$.

Ist statt des Meridians eine beliebige Kurve

Fig. 130 .
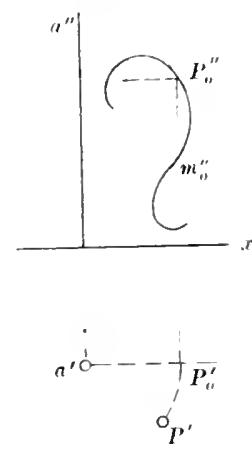

Fig. 131.

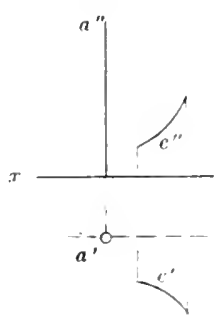
$c\left(c^{\prime} c^{\prime \prime}\right)$ als Erzeugende der Fläche gegeben, so erhalten wir $m_{0}$, indem wir für eine Reihe ron Punkten auf $c$ die durch sie gehenden Parallelkreise in Grund- und Aufrib zeichnen (Fig. 131).

136. Aufgabe. Den Schnitt einer Undrehungsfläche. deren Achse $a \perp \Pi_{1}$ is $\mathrm{t}$, mit der Ebene $\mathrm{E}\left(e_{1} e_{2}\right)$ zu konstruieren 
(Fig. 132). Die schuittkure $\mathrm{k}$ ist symmetrisch in bezug auf die Schnittlinie $f$ ron $\mathrm{E}$ mit der anf $e_{1}$ senkrechten Moridianebene $\mathrm{M}$, ihr Grundrib $k^{\prime}$ also symmetrisch in bezug anf das Lot $f^{\prime}$ von $a^{\prime}$ anf $e_{1}$. Um $f^{\prime}$ zu ermittelu, bestimmen wir den Schnittpunkt $A$ von a mit $\mathrm{E}$ : Die Ebene $M_{0}$ des Cmribmeridians $m_{0}$ schneidet $E$ in einer zweiten Hauptlinie $h$, und diese trifft $a$ in . 1 . Damn ist $f$ die Verbindungshinie von A mit dem Schnittpunkte $B$ von $f^{\prime \prime}$ und $e_{1}$. - Anf $f$ befindet sich der höchste und der tiefste Punkt der Kurve $h_{\text {; }}$ sie sind die Schuittpunkte $C$ und $D$ von $f$ mit der in $M$ liegenden Meridiankurve $m$. Wir konstruieren zunächst diese ausgezeichneten Punkte von $k$, indem wir die Ebene $M$ mm a drehen, bis sie mit $M_{0}$ zusammenfällt. [Yabei gelangt im

Fig. 132.

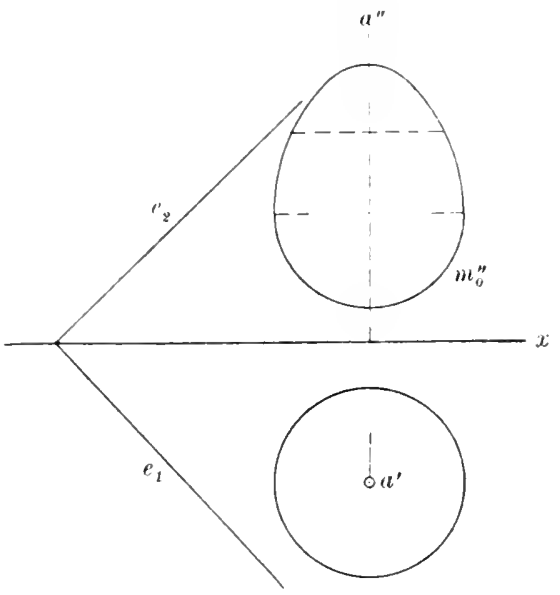

Fig. 133.
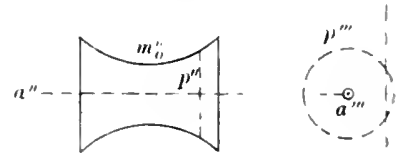

nach $m_{0}, B$ nach $B_{0}$, und die Gerade $A^{\prime \prime} B_{0}^{\prime \prime}$ schneidet $m_{0}^{\prime \prime}$ in $C_{0}^{\prime \prime}$ und $D_{0}^{\prime \prime}$. Hieraus ergibt sich z. B. $C^{\prime}$ mittels $a^{\prime} C^{\prime \prime}=$ Abstand $C_{0}^{\prime \prime} a^{\prime \prime}$. Die Tangenten von $l i$ in $C$ und $D$ sind $\| e_{1}$.

Weitere Punkte von $l_{i}$ werden mittels horizontaler Hilfsebenen gefunden, die zwischen $C$ und 7 ) beliebig angenommen werden. Eine solche Ebene schneidet die Fläche in einem Parallelkreise $p$, die Ebene $E$ in einer ersten Hauptlinie, und diese trifft $p$ in zwei Pnnkten $I^{\prime}$ und $Q$ von l: In der angegebenen Weise ermitteln wir u. a. die Punkte von $l_{i}$ im ersten Umrib der Flache: die Pnnkte im zweiten Umrib liegen auf der Geraden $h$.

Dip 'Tangente von $k$ im Punkte $P$ ist die Schnittlinie von E nit der Berührungsebene der Fläche in $P$. Dabei genügt es, von dieser Beriihrungsebene entweder die erste $S_{p} u r$, oder die in $M_{0}$ liegende Hauptlinia zn konstruieren.

In Fig. 133 ist $a \| s$ und $E \| \Pi_{2}$, es handelt sich also mu $\mathrm{mm}$ dits Konstruktion vou li". Die zu $\Pi_{1}$ parallele Neridiankurve $m$ liefert sofort die auf $a^{\prime \prime}$ liegenden Punkte $C^{\prime \prime}, D^{\prime \prime}$. Die Punkte anf einem beliebigen Parallelkreise $p$ ergeben sich mittels eines Seitemrisses anf eine $\Pi_{3} \perp a$; dabei ist Abstand $e_{3} a^{\prime \prime \prime}=$ Abstand $e_{1} a^{\prime}$. 
137. Schnitt einer Undrehungsfläche mit einer ihrer. Berïhrungsobenen (Fig. 134). Ist die Meridiankurve m im Punkte I'konkar gegen die Achse $a$. so hat die Berührungsebene $T$ lieses Punktes in seiner nächsten Umgebung keinen Punkt mit der Fläche gemein. Sie schueidet die Fläche in ihrem weiteren Verlanfe in einer Kurre k, welcher der Punkt $P$ als isolierter Punkt angehört. Denken wir uns die Ebene $T$ parallel zu sich selbst ein wenig in das Innere fer Fläche verschoben, so hat sie mit der Fläche in der Lmgebung von $P$ ein kleines (Oval gemein. Der Punkt $P$ heibt deshalb ein elliptischer. Punkt ler Fläche. Da er als Bestandteil von $k$ als eine anf eineu Punkt zusammengeschrumpfte Ellipse betrachtet werden kann, so hat jede in cler Ebene $T$ durch ihn gezogene Gerade an dieser Stelle mit $l$ zwei zusammenfalleude Punkte gemein: in riesem Sinne ist er also ein Doppelpunkt von li.

Ist ferner $m$ im Punkte $Q$ konvex gegen $a$, so liegen $m$ und der Parallelkreis des Punktes $Q$ auf verschiedenen Seiten der zugehörigen Berïhrungsebene; diese trifft jeden der beiderseits benachbarten Parallelkreise in zwei Punkten und schneidet demnach die Fläche in einer Kurve, die in $Q$ einen Knoten-

Fig. 134.

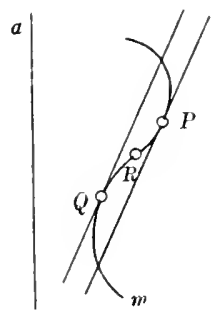
punkt hat. Die der Berührungsebene beiderseits benachbarten Parallelschnitte der Fläche haben in unmittelbarer Nähe von $Q$ das Aussehen einer Hyperbel in der nächsten Imgebung ihrer Scheitel: wir nennen daher $Q$ einen hyperbolischen Punkt der Fläche.

Konstruieren wir endlich die Berübrungsebene im Wendepunkte $R$ von $m$, so schneidet diese Ebene von den Parallelkreisen der Nachbarpunkte nur diejenigen, rie unterhalb des zu $R$ gehörigen Parallelkreises liegen; infolge Zusammenziehung der im vorigen Falle erhaltenen Schleife wird also $R$ zu einem Rückkehrpunkte der Schnittkurve (parabolischer. Flächenpunkt).

Überlaupt gilt für jede krumme Fläche der Satz: Die Berührungsebene schneidet die Fläche in einer (reellen orler imaginären) Kurve, die im Berührungspunkte einen Doppelpunkt hat. Je nachdem dieser ein isolierter. Punkt, oder ein Knotenpunkt, oder ein Rückkehrpunkt der Schnittkurve ist, nennen wir ihn einen elliptischen, hyperbolischen oder parabolischen Punkt der Fläche (vgl. hiermit den Satz in Art. 114).

\section{Das einschalige Umdrehungshyperboloid.}

138. Wir untersuchen die Lmdrehungsfläche $\Phi$, die entsteht, wenn eine Gerarle $g \mathrm{~mm}$ eine zu ihr windschiefe Achse a rotiert. Dabei sei $a \perp \Pi_{1}$ und die Erzengende $g$ in ihrer Anfangslage $\| \Pi_{2}$ (Fig. 135).

Je zwei Lagen ron $g$ sind windschief zueinander: denn sie begrenzen auf allen Parallelkreisen Bogenstücke von gleichem Zentriwinkel und könneu sich daher nirgends schneiden. Die Fläche $\phi$ ist also windschief.

Der Punkt $K$ von $g$, der $a$ am nächsten liegt, heschreibt den Kehlkreis $l_{i}$ der Fläche. Zwei Punkte $B$ und $C$ von $g$, die von $K^{-}$ 
Heich weit entfernt sind. durchlaufen gleiche Parallelkreise $b$ und $c$; die Fläche $\Phi$ ist daher symmetrisch in bezug auf die Kehlkreisebene.

U'm eine Anzahl von Erzengenden der Fläche zu zeichnen, teilen wir die Kreise $b$ und $c$ von $B$ und $C$ ans in $n$ gleiche 'Teile und ver-

Fig. 135.
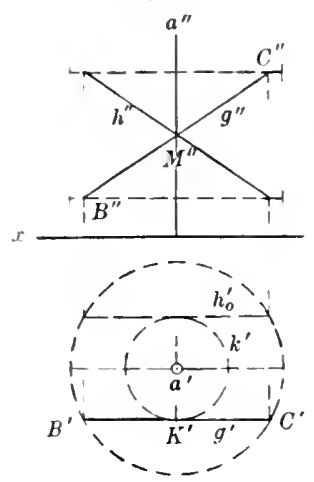
binden die entsprechenden 'Teilpunkte durch Geraden. Wählen wir den Radius von $b$ und $c$ so, dab $-B^{\prime} a^{\prime} C^{\prime}$ ein ganzes Vielfaches von $360^{\circ}$

$n$

wie den Radius von $k$. - , so erreichen wir den Vorteil, daß die beiden T'eilungen im Grundriß zusammenfallen.

Die ersten Projektionen der Erzengenden berühren den Kreis $k^{\prime}$, ilıre zweiten Projektionen den Aufriß des Umrißmeridians $m_{0}$.

139. Ziehen wir durch $K$ die Gerade $h$ symmetrisch zu $g$ in bezug auf die Kehlkreisebene, so liegt der untere 'Teil von $h$ als Spiegelbild des oberen 'Teiles ron $g$ auf dem Spiegelbilde der oberen Hälfte der Fläche $\Phi$, d. h. auf der unteren Hälfte dieser Fläche. Die Gerade $h$ liegt demnach ganz auf $\Phi$, und wir können folglich die Fläche auch durch Drehung von $h$ um $a$ erzeugen. Auf der Fläche befindet sich also eine zweite Schar gerader Linien. Durch jeden Punkt der Fläche gehen zwei Geraden, nämlich je eine von jeder Schar. Jede Gerade der einen Schar schneidet alle Geraden der anderen Schar, aber keine Gerade derselben Schar.

Durch eine halbe Tmdrehung um $a$ gelangt $h$ in die Lage $h_{0} \| g\left(h_{0}^{\prime} \| g^{\prime}, h_{0}^{\prime \prime}=g^{\prime \prime}\right)$. Daraus folgt: $Z_{u}$ jeder Geraden der einen Schar gibt es eine parallele Gerade in der anderen.

140. T'm die Ordnung der Fläche $\Phi$ zu bestimmen, verbinden wir irgend zwei Punkte $I^{\prime}$ und $Q$ von $\Phi$ durch eine Gerade $i$ und fragen nach der Anzahl der Schnittpunkte, die $i$ mit $\Phi$ gemein hat. Durch $P$ gehen zwei Geraden $g_{1}$ und $h_{1}$ der Fläche, ebenso durch $Q$ zwei Geraden $g_{2}$ und $h_{2}$. Nach dem Vorlergehenden schneiden sich $g_{1}$ und $h_{2}$ in einem Flichenpunkte $T$, liegen also in einer Ebene T. Diese hat mit der $\Phi$ nur die Geraden $g_{1}$ und $h_{2}$ gemein, denn alle Lagren der Erzengenden $g$ schneiden $\mathrm{T}$ auf $h_{2}$, und alle Lagen von $h$ treffen $\mathrm{T}$ in Punkten von $g_{1}$. Die Gerade $i$ enthält also außer $P$ und $Q$ keine weiteren Punkte von $\Phi$, d. h. $\Phi$ ist eine Fläche zweiter Ordnung.

Der Meridian $m_{0}$ der Fläche ist demnach ein Kegelschnitt mit der Achse $a$. Da ferner $g$ und $h$ zur Ebene $\mathrm{M}_{0}$ parallel sind, so ist $m_{0}$ eine Hyperbel; ihre Asymptoten gehen durch den Mittelpunkt $M$ des Keblkreises $k$ parallel zu $g$ und $h$, und ihre Scheitel liegen auf $h$. Die Fläche $\Phi$ istalso ein einschaliges Umdrehungshyperboloid. Die Asymptoten ron $m_{0}$ erzeugen den Asymptotenkegel der Fläche.

Jede Gerade der vorhin betrachteten Ebene $T$ schneidet das Hyperboloid in den beiden Punkter, die sie mit $g_{1}$ und $h_{2}$ gemein hat. 
Alle durch ' $T$ ' gehenden Geraden ron $T$ sind mithin 'Tangenten der Fläche, folglich ist $T$ die Berührungsebene in $T$, d. h.: Die Berïhrungsebene in irgend einem Pnnkte des Hyperboloids ist die Ebene der beiden durch den Punktgehenden Erzeugenden. Das einschalige Umdrehungshyperboloid hat demnach lauter hyperbolische Punkte.

$Z$ wei parallele Erzengende, wie $g$ und $h_{0}$, bestimmen eine asymptotische Berührungsebene der Fläche. Diese Ebene berührt gleichzeitig den Asymptotenkegel in der zu beiden Erzengenden parallelen Mantellinie.

Jede nicht berührende Ebene schneidet das Hyperboloid in einer Ellipse, Parabel oder Hyperbel, je nachdem sie zu keiner, einer oder zwei Mantellinien des Asymptotenkegels parallel ist.

141. Einschalige Umdrehungshyperboloide, diesich längs piner Erzeugenden berühren. Da die Berührungsebene des Hyperboloids $\Phi$ in irgend einem Punkte $P$ von $g$ durch diese Gerade geht, so ist die Flächennormale in $P$ das Lot zu $g$, das die Achse $a$ schneidet (Art. 131). Stellen wir $g \perp \Pi_{1}$ und $a \| \Pi_{2}$, so erhalten wir als Aufriß der Normale in $P$ die Gerade $P^{\prime \prime} Q^{\prime \prime} \| x$, die $a^{\prime \prime}$ in $Q^{\prime \prime}$ schneidet, und hierzu als Grundric die Gerade $g^{\prime} Q^{\prime}$ (Fig. 136).

Wir betrachten jetzt ein zweites Hyperboloid $\boldsymbol{\psi}$, das mit $\Phi$ die Erzeugende $g$ gemein hat, und dessen Achse $b$ den kürzesten Abstand $K M$ von $g$ und $a$ in $N$ rechtwinklig schneidet. Die beiden Flächen haben sowohl in $K$, als auch im unendlich fernen Punkte von $g$ dieselbe Berührungsebene, nämlich in $K$ die Ebene durch $g \| \Pi_{2}$ und im unendlich fernen Punkte von $g$ die auf dieser

Fig. 136.
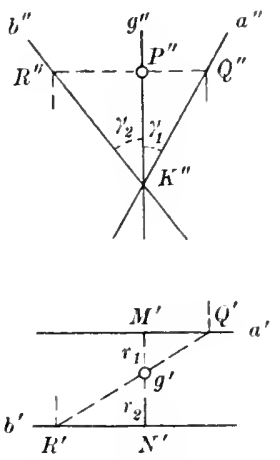
senkrechte Ebene durch $g$ und $M I$. Sollen auch die Berührungsebenen in $P$ miteinander zusammenfallen, so muB die Normale $P R$ von $\psi$ die Verlängerung der Normale $P Q$ bilden. es muL sich also verhalten

$$
g^{\prime} M^{\prime}: g^{\prime} N^{\prime}=M^{\prime} Q^{\prime}: N^{\prime} R^{\prime}=P^{\prime \prime} Q^{\prime \prime}: P^{\prime \prime} R^{\prime \prime} .
$$

Bezeichnen wir die Kehlkreisradien $K M$ und $K N$ mit $r_{1}$ und $r_{2}$ und die von $g$ mit $a$ und $b$ gebildeten Winkel mit $\gamma_{1}$ und $\gamma_{2}$, so ergibt sich

$$
r_{1}: r_{2}=\operatorname{tg} \gamma_{1}: \operatorname{tg} \gamma_{2} \text {. }
$$

Da hier die Entfernung des Punktes $I$ von $K$ nicht vorkommt, so berühren sich die Hyperboloide in allen Punkten von $g$, sobald die Bedingung 1) erfüllt ist. Dann können wir aber die beiden Flächen auf unzählig viele Arten berührend aneinander legen, nämlich so, daß eine beliebige Erzeugende des einen Hyperboloids mit einer beliebigen des anderen zusammenfällt. Dazu ist nur erforderlich, dab die kürzesten Abstände der Erzeugenden von den Achsen in eine Gerade fallen.

142. Betracbten wir die Hyperboloide $\phi$ und $\psi$, die sich längs der Erzeugenden $g$ berühren, als Oberflächen zweier widerstandsfähigeı Körper, so können wir sie benutzen, um eine Drehung nm die 
Achse a anf die dazu windschiefe Achse b proportional zu ubertragen, wie dies in ähnlicher. Weise bei parallelen Achsen durch zwei Lundrehungszylinder, bei sich schneidenden Achsen durch zwei Indrehungskegel erreicht wirl. Ist die Reibung zu gering, so müssen beide Körper längs solcher Erzeugenden, die miteinander parweise zur Teckung gelangen. mit Zähnen versehen werden (vgl. hierzu auch Art. 80).

Es fragt sich noch, in welchem Verhältnis die Limdrehungsgeschwindigkeit von $\Phi$ auf $\psi$ übertragen wird.

Sei $y_{1}$ die Erzengende von $\Phi$, die infolge der unendlich kleinen Drehung $l \varphi$ un $a$ an die Stelle ron ! tritt, $g_{2}$ die entsprechende

Fig. 137.

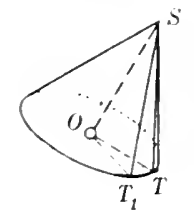
Erzengende von $\psi$. Da die durch /f und $g_{1}$ und durch $g$ und $g_{2}$ begrenzten windschiefen Flächenelemente ron $\Phi$ und $\psi$ miteinander zur leckung kommen, so bilden $g$ und $g_{2}$ denselben unendlich kleinen Winkel $d \vartheta$, wie $g$ und $g_{1}$. Ziehen wir durch irgend einen Punkt s Parallelen zu den Erzeugenden von $\Phi$, so entsteht ein gerader Kreiskegel mit der Achse $S O \|$ “; eine zur Achse senkrechte Ebene schneide diese in $O$ und die $z n g$ und $g_{1}$ parallelen Mantellinien in $T$ und $T_{1}$. In der so erhaltenen Fig. 13T ist - TSO $=-T_{1} S O=\gamma_{1}:-T S T_{1}=1 \%$ nud - TOT $=d \varphi$, mithin

$$
T T_{1}=O T \cdot d \varphi .
$$

Andererseits ist aber

folglich

$$
T T_{1}=S T \cdot d \vartheta=\frac{O T}{\sin \gamma_{1}} d \boldsymbol{\theta}
$$

$$
d \theta=d \varphi \sin \gamma_{1}
$$

Verstehen wir unter $d \psi$ die unendlich kleine l)ehung nu $b$; die $g_{2}$ in die Lage $g$ bringt, so finden wir ebenso

demnach ist

$$
d \vartheta=d \psi \sin \gamma_{2}
$$

$$
d \varphi \sin \gamma_{1}=d \psi \sin \gamma_{2} .
$$

Dreht sich nun $\Phi$ 11 a mit der konstanten Geschwindigkeit $\omega_{1}$ und infolgerlesien $\boldsymbol{\psi}$ umb mit der noclı unbekannten Geschwindigkeit $\omega_{2}$, so verhält sich

$$
\omega_{1}: \omega_{2}=d \varphi: d \psi:
$$

mithin besteht zwischen den Lmdrehnngsgeschwindigkeiten die Beziehnng

$$
\omega_{1}: \omega_{2}=\sin \gamma_{2}: \sin \gamma_{1}
$$

143. Dies führt zur Lösung der Aufgabe: Zwe L lmal rehungshyperboloidemit gegebenen, zueinander windschiefen Achsen a und b zu konstruieren, welche lie llreling nm a auf die Achse $b$ in der Weise übertragen, daf die Lindrehungsgeschwindigkeiten $\omega_{1}$ und $\omega_{2}$ in einem qegebenen Verhältni: stehen. In Fig. 138 in $a \perp \Pi_{1}$ mul b $\| \Pi_{2}$. und e. möge sich verhalten $\omega_{1}: \omega_{2}=1: 2$. 
Die gemeinsame Erzeugeule $g$ der beiden Hyperboloide steht senkrecht auf der kürzesten Entfernung $M N$ von $a$ und $b$, ist also gleichtalls $\| \Pi_{2}$. Ihr Aufrib ergibt sich zufolge der Bedingung 2), indem wir einen Punkt ermitteln, dessen Abstände von $a^{\prime \prime}$ und $b^{\prime \prime}$ sich verhalten wie $\stackrel{2}{:} 1$. Legen wir dam die strecke $M N$ senkrecht zu $g^{\prime \prime}$ zwischen $a^{\prime \prime}$ nurl $\ell^{\prime \prime}$, so sind die auf ihr durch $g^{\prime \prime}$ elzeugten Abschnitte nach 1) bzw. gleich den Kehlkreisradien $r_{1}$ und $r_{2}$.

Um auf den so bestimmten $\mathrm{H}_{y}$ perboloiden $\phi$ und $\psi$ eine Anzahl ron Erzengenden anzugeben, die bei del Übertragung rler Drehung parweise zusammenfallen, wählen wir auf der Geraden $g$ in gleichen Abständen von ihrem Schnittpunkte mit $M N$ zwei Punkte und begrenzen die beiden Flächen durch die Parallelkreise, die durch diese Punkte gehen. Da die

Fig. 13 .

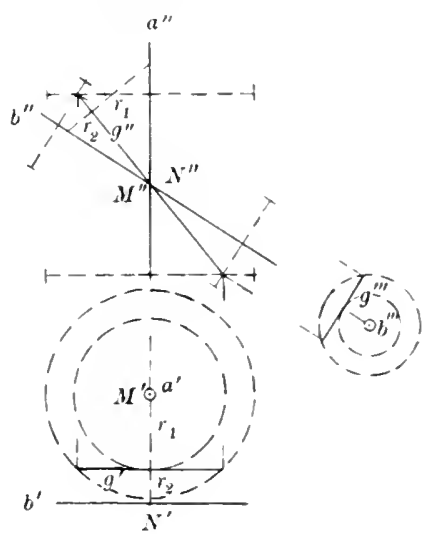
Lmdrehungsgeschwindigkeiten von $\Phi$ und $\psi$ sich wie $1: 2$ verhalten sollen, teilen wir die auf $\phi$ liegenden Kreise von $g$ ans in doppelt so viel gleicher Teile wie die Kreise anf $\psi$.

Lmriblinien und Schattengrenzen.

144. Anfuabe. Den ersten scheinbaren Umrib einer L'mdrehungsfläche zu konstruieren, deren Achsezu $\Pi_{2}$ parallel, aber gegen $\Pi_{1}$ beliebig geneigt ist (Fig. 139). Die Fläche sei gegeben durch die Achse $a$ und den $z u \Pi_{2}$ parallelen Meridian $m_{0}$, der wie vorher den zweiten Lmriß liefert. Als ersten wahren Cmriß erhalten wir im allgemeinen eine Raumkurve $u$. $\quad$ Im diejenigen Punkte rou $u$ zu ermitteln, die sich auf einem beliebig gewählten Parallelkreise $p$ befinden. benutzen wir den Kegel und die Kugel, die die Fläche längs $p$ berührell. In jedem sichnittpunkte ron $p$ und $u$ haben die drei Flächen eine gemeinsame Berührungsebene, die auf $\Pi_{1}$ senkrecht steht: ihre ersten scheinbaren $\mathrm{Lm}$ riblinien berübren sich daher im Grundric dieses Schnittpunktes (Art. 93). Ziehen wir im Endpunkte $P_{0}^{\prime \prime}$ der Strecke $p^{\prime \prime}$ an $m_{0}^{\prime \prime}$ die Tangente $P_{0}^{\prime \prime} S^{\prime \prime}$ und die Normale $P_{0}^{\prime \prime} O^{\prime \prime}$, so erhalten wir auf $a$ die Spitze $S$ res Be-

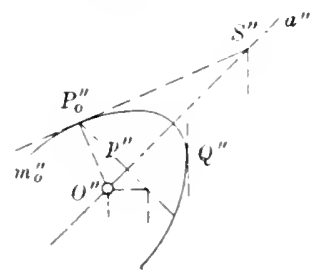
rührungskegels und den Jlittelpunkt $O$ der zugehörigen Kugel. Der erste scheinbare Lmrib cler Kugel ist der Kreis $h^{\prime}$ um $O^{\prime}$ mit dem Radius $O^{\prime \prime} P_{0}^{\prime \prime}$; die entsprechenden Lmrißlinien des Kegels sind also die 
'Tangenten aus $S^{\prime}$ an $h^{\prime}$. Sie berühren $h^{\prime}$ und zugleich die gresuchte Kurve $u^{\prime}$ in zwei Punkten $T^{\prime}$ und $U^{\prime}$.

Der Punkt $S$ ist zur Bestimmung von ' $T$ ' und $U$ nicht orforderlich. Der Aufrib des Kreises $h$ fält nämlich in die Parallele durch $O^{\prime \prime}$ zu $x$, und liese schneidet $p^{\prime \prime}$ in $T^{\prime \prime}=U^{\prime \prime}$.

Dem Punkte $Q^{\prime \prime}$ vou $m_{0}^{\prime \prime}$, dessen 'Tangente $\perp x$ ist, entspricht auf $a^{\prime}$ der Scheitel $Q^{\prime}$ von $u^{\prime}$. Der erste scheinbare Umrib der zugehörigen Berührungskugel ist der Krümmungskreis ron $u^{\prime}$ in $Q^{\prime}$. Die Parallelkreise der Fläche, die zwischen dem Punkte $Q$ und dem Scheitel der Fläche liegen, enthalten keine Punkte von $u$.

Ist a zu keiner Projektionsebene parallel, so finclet man den ersten und den zweiten scheinbaren Umril mittels einer $\Pi_{3}$, die zu einer projizierenden Ebene von $a$ parallel ist.

145. Ist die Meridiankurve $m_{0}$ der vorigen Aufgabe ein Kreis um $\boldsymbol{K}_{0}$ mit dem Ralius $r$, so erzeugt sie eine Ringfläche (Wulstfläche, Torus), und dann läbt sich die Unriblinie $u^{\prime}$ noch einfacher koustruieren (Fig. 140). Die Ringfläche ist nämlich die Einhüllende

Fig. 140.
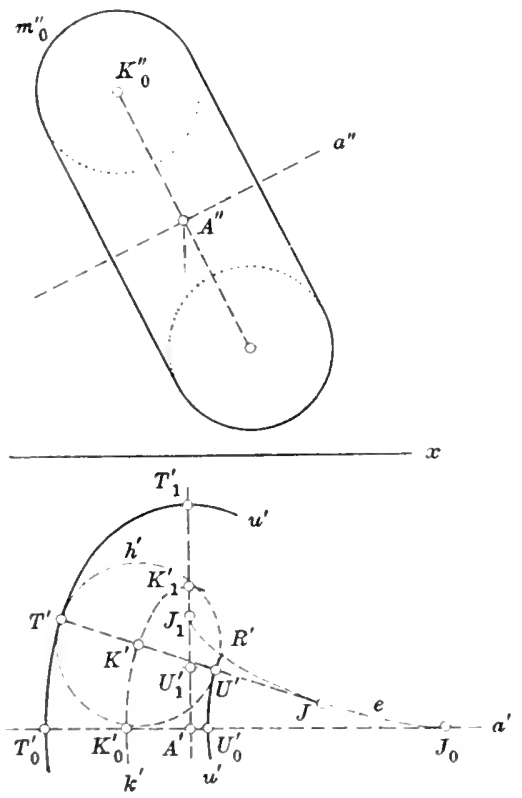
einer um a rotierenden Kugel vom Mittelpunkt $K_{0}$ und dem Radius $r$, folglich ist $u^{\prime}$ die Einhüllende der ersten scheinbaren Umrisse aller Lagen dieser Kugel. Bei der Drehung um $a$ beschreibt $K_{0}$ einen Kreis $k$, dessen Mittelpunkt $A$ auf $a$ liegt. Sein Grundrib ist eine Ellipse $l_{i}^{\prime}$ mit den Halbachsen $A^{\prime} K_{0}^{\prime}$ und $A^{\prime} K_{1}^{\prime \prime}=A^{\prime \prime} K_{0}^{\prime \prime}$. Wir erhalten demnach $\iota^{\prime}$ als die Einhüllende aller Kreise vom Radius $r$, deren Mittelpunkte sich auf $k^{\prime}$ befinden. Irgend zwei dieser Kreise, mit den Mittelpunkten $\boldsymbol{K}^{\prime}$ und $L^{\prime}$, schmeiden sich in zwei Punkten ' $T$ ' und $U^{\prime}$, deren Verbindungslinie von $K^{\prime} L^{\prime}$ senkrecht halbiert wird. Sind nun $K^{\prime}$ und $L^{\prime}$ zwei unendlich benachbarte Punkte von $k^{\prime}$, so wird $T^{\prime} U^{\prime}$ zur Normale von $k^{\prime}$ in $K^{\prime}$; dann ist $K^{\prime} T^{\prime}=K^{\prime} U^{\prime}=r$, und $T^{\prime}$ und $U^{\prime}$ sind zwei P'unkte von $u^{\prime}$. Wir können daher die Kurve $u^{\prime}$ auch in der Weise konstruieren, daß wir auf allen Nornalen von $k^{\prime}$ beiderseits die Strecke $r$ abtragen; d. h. $u^{\prime}$ ist die Parallelkurve der Ellipse $k^{\prime}$ in Abstande $r$.

Die Cmrißlinie $u$ besteht demnach aus zwei Teilen; der eine liegt im Gebiet der olliptischen, der andere im Gebiet der hyperbolischen Punkte des Ringes. 
Der Kreis $h^{\prime}$, der $K^{\prime}$ zum Mittelpunkt und $r$ zum Radius hat, berührt $u^{\prime}$ in $T^{\prime}$ und $U^{\prime}$; die Ellipsennormale $T^{\prime} U^{\prime}$ ist also zugleich Normale von $u^{\prime}$ in $T^{\prime}$ und $U^{\prime}$. Die Kurven $k^{\prime}$ und $u^{\prime}$ haben daher dieselbe Evolute $e$, folglich in entsprechenden Punkten denselben Krümmungsmittelpunkt. Besonders nützlich bei der Konstruktion von $u^{\prime}$ siud die Scheitelkrümmungsmittelpunkte $J_{0}, J_{1} \ldots$ von $k^{\prime}$.

Vir können die beiden Teile von $u$ ' auch als die Bahnkurven auffassen, welche die Punkte $T_{0}^{\prime}$ und $U_{0}^{\prime}$ beschreiben, während die Gerade $J_{0} T_{0}^{\prime}$ auf der Evolute $e$ rollt. Trifft bei dieser Rollung der Punkt $U_{0}^{\prime}$ auf $e$, so entsteht ein Rückkehrspunkt $R^{\prime}$ von $u^{\prime}$. Wir erhalten also $R^{\prime}$; indem wir auf $e$ den Bogen $J_{0} R^{\prime}$ angenähert gleich der Strecke $J_{0} U_{0}^{\prime}$ machen. In Punkte $R$ wird die Raumkurve $u$ vom projizierenden Stralıl $R R^{\prime}$ berührt; hier endigt der sichtbare Bogen des inneren 'Teiles von $u$. Jeder tiefer liegende Punkt des inneren Kurvenstückes ist nämlich im Grundriß unsichtbar, weil der projizierende Strahl, der in dem betreffenden Punkte die Ringfläche berührt, die undurchsichtig gedachte Fläche bereits oberhalb der Berührungsstelle schneidet.

Man zeichne von der Kurve $u$ auch den Aufrib mittels der zweiten Projektionen der Punkte $K \ldots$ und der horizontalen Kugelkreise $h \ldots$

146. Aufgabe. Bei einer Umdrehungsfläche, deren Achsea $\perp \Pi_{1}$ ist, die Eigenschattengrenze $s$ und den Schlagschatten auf die $\Pi_{1}$ für Parallelbelenchtung zu konstruieren (Fig. 141). Die Kurve $s$ ist symmetrisch in bezug auf die zur Lichtrichtung parallele Meridianebene M, ihr Grundriß also symmetrisch in bezug auf die Gerade $l^{\prime}$, die in der Richtung der ersten Projektionen der Lichtstrahlen durch $a^{\prime}$ gezogen wird. WVir ermitteln zunächst die in der Ebene $M$ befindlichen Punkte von $s$, indem wir an die zugehörige Meridiankurve $m$ in der Lichtrichtung Tangenten legen. $\mathrm{Zu} \mathrm{dem}$ Zwecke ziehen wir in $M$ durch irgend einen Punkt $A$ von $a$ den Lichtstrahl $l$ und drehen die Ebene M um ", bis sie mit der Ebene $M_{0}$ des Umrißmeridians $m_{0}$ zusammenfällt (vgl. Art. 87). Bezeichnen wir mit $l_{0}^{\prime \prime}$ den Aufriß des gedrehten Lichtstrahls, mit $C_{0}^{\prime \prime}$ und $D_{0}^{\prime \prime}$ die Punkte von $m_{0}^{\prime \prime}$, deren 'Taugenten $\| l_{0}^{\prime \prime}$ sind, so entsprechen ihnen auf $m$ als höchster und tiefster Punkt von $s$ die Punkte $C$ und $D$; dabei ist $a^{\prime} C^{\prime}=$ Abstand $C_{0}^{\prime} a^{\prime \prime}$ und $C_{0}^{\prime \prime} C^{\prime \prime} \| x$.

Fig. 141.
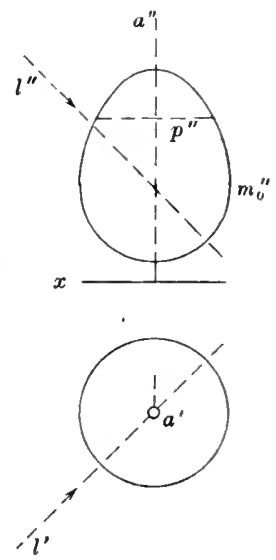

Die Punkte, die ein zwischen $C$ und $D$ liegender Parallelkreis $p$ mit $s$ gemein hat, können auf zweierlei Weise bestimmt werden:

a) Kegelverfahren. Die gesuchten Punkte $P$ und $Q$ liegen auch auf der Eigenschattengrenze des Kegels, der die Tmdrehungsfläche in $p$ berührt. Konstruieren wir also von der Spitze $S$ dieses Kegels den Schatten $S_{1}$ anf die Ebene von $p$, so sind $P^{\prime}$ und $Q^{\prime}$ die Berührungspunkte der Tangenten aus $S_{1}^{\prime}$ an $p^{\prime}$. - Diese Konstruktion versagt, wenn $S$ unerreichbar ist. Deshalb empfiehlt es sich, alle Berührungskegel, die zu den verschiedenen Parallelkreisen der Fläche gehören, 
parallel zu sich zu verchieben, so daß ilure spitzen in denselben Punkt $A$ von a fallen, und die verschobenen Kegel mit der $\Pi_{1}$ zu schneiden. Dann liegen die Grundribspuren ihrer Eigenschattengrenzen auf dem Kreise über den Durchmesser $a^{\prime} A_{h}$. In Grundriß decken sich aber die Eigenschattengrenzen jedes hegels vor und nach der Terschiebung.

b) Kugrelverfahren. Die Punkte $P$ und $Q$ befinden sich fernel auf der Eigenschattengrenze der zum Parallelkreise l' gehörenden Berührungskugel. Bezeichnen wir mit $O$ den Mittelpunkt dieser hugel, mit $E$ die Ebene ihrer Eigenschattengrenze, so geht $E$ durch $O \perp l$ und schneidet die Ebene $M_{0}$ in einer Geraden $O R$, deren Aufriß auf $l^{\prime \prime}$ senkrecht steht. Bedentet $R$ den Schnittpunkt vou $O R$ mit der Ebene von $p$, so lient $R^{\prime}$ auf der Parallele durch $a^{\prime}$ zu $x$. Dann schneidet $\mathrm{E}$ die Ebene von $p$ in der durch $R$ gehenden Geraden $P Q$ : ihr Grundriß ist das Lot von $R^{\prime}$ auf $l^{\prime}$.

Wir ermitteln insbesondere von der Kurve $s$ die Punkte $T$ und $U$ im ersten, sowie $T$ und $W$ im zweiten Lmrif der Fläche, indem wir an die scheinbaren Umrißlinien 'Tangenten ziehen, die bzw. zu l' und $l^{\prime \prime}$ parallel sind.

Für Licht in der Richtung der. Würfeldiagonale ergeben sich wesentliche Tereinfachungen der soeben abgeleiteten honstruktion. I ann ist $l_{0}^{\prime \prime}$ parallel zur Hypotenuse eines rechtwinkligen Dreiecks, dessen Katheten zu $x$ parallel bzw. senkrecht sind und sich wie $\sqrt{2}: 1$ verbalten. Wir finden also die Punkte $C_{0}^{\prime \prime}, D_{0}^{\prime \prime}$ und hieraus $C^{\prime \prime}, D^{\prime \prime}$ obne Benutzung des Grundrisses; denn es ist Abstand $C^{\prime \prime} a^{\prime \prime}$ gleich der Fathete eines gleichschenklig rechtwinkligen Dreiecks, das die Entfermung des Punktes $C_{0}^{\prime \prime}$ ron $a^{\prime \prime}$ zur Hypotenuse hat. Ebenso erhalten wir ron $s^{\prime \prime}$ die Punkte $T^{\prime \prime}, C^{\prime \prime}$. Bezeichnen wir ferner mit $Z$ denjenigen Punkt von $s$, der sich auf dem Parallelkreise des Punktes II befindet. so ist $Z^{\prime \prime}$ der Schnittpunkt von $a^{\prime \prime}$ mit der Parallele zu ${ }^{\prime}$ durch $W^{\circ \prime}$ : denu $Z^{\prime}$ liegt symmetrisch zu $W^{-\prime}$ in bezug auf $l^{\prime}$, fällt also auf die Verlängerung ron $a^{\prime \prime}$. - Die fünf Punkte $V^{\prime \prime}, T^{\prime \prime}, Z^{\prime \prime}, D^{\prime \prime}, W^{\prime \prime}$ genügen in manchen Fällen zur angenäherten Bestimmung des sichtbaren Teils von $s^{\prime \prime}$.

Der Lichtstrahlenzylinder, der die Fläclie in $s$ berührt, schueidet die $\Pi_{1}$ in der Schlagschattengrenze $s_{h}$, die in bezug auf $i^{\prime}$ symmetriscl ist. Wir konstruieren sie aus dem Schatten der vorher benutzten Parallelkreise um der auf ihnen liegenden Punkte von s. Ist $\boldsymbol{M}$ der Jittelpunkt des Parallelkreises $p$, so finden wir anf clem Kreise $p_{h}$ un $I_{h}$ die I'unkte $P_{h}$ und $Q_{h}$ ron $s_{h}$ mittels der Parallelen durch $M_{h}$ zu $a^{\prime} P^{\prime}$ und $a^{\prime} Q^{\prime}$, und danu berührt $p_{h}$ die Kurve $s_{h}$ in $P_{h}$ und $Q_{h}$. Daher ist $M_{h} Y_{h}$ die Normale von $s_{h}$ in $I_{h}$. - Der Schatten des durch den höhnten Punkt $C$ von $s$ gehenden l'arallelkreises hat mit $s_{h}$ in $C_{h}$ zwei zusammenfallende Berïhrungspunkte, l. h. vier unendlich nahe Punkte gemein: er ist daher der hrümmungskreis der hurve $s_{h}$ in ibrem scheitel $C_{h}$. Ebenso ist der Krümmungsradius ron $s_{h}$ in $D_{h}=\mathrm{Ab}$ stand $D_{u}^{\prime \prime} a^{\prime \prime}$.

147. Dieselbe Aufgabe für die Ringfläclie (Fig. 1+2). Wir bezeichnen wieder mit $K_{0}$ den Mittelpunkt, mit $r$ den Radius des 
Meridiankreises $m_{0}$, mit $A$ den Mittelpunkt des Ringes, d. h. den FuLpunkt des Lotes von $K_{0}$ auf $a$, und setzen $A K_{0}=d$. Beschreiben wir un $A$ mit dem Radins $r$ eine Kugel, so schneidet eine durch a gelegtr Ebene die Kugel in einem Hauptkreise $i$, den Ring in zwei gleich großen lireisen $m_{1}$ und $m_{2}$, und dann fällt der Kreis $i$ mit $m_{1}$ oder $m_{2} z a-$ sammen, wenn wir ihn $\perp^{\prime \prime}$ nach der einen oder anderen Seite um dit strecke $d$ verschieben. Dabei gelangt ein beliebiger Punkt $P$ von , bzw. nach $P_{1}$ auf $m_{1}$ oder nach $P_{2}$ auf $m_{2}$, so dab $P P_{1}=P P_{2}=d$ und $\perp a$ ist. Da die Tangenten an $i, m_{1}, m_{2}$ in $P, P_{1}, P_{2}$ parallel sind, so gilt dasselbe von den Berührungsebenen der Kugel und des Ringes in den grenawnten Punkten; ist also $I^{\prime}$ ein Punkt der Eigenschattengrenze \& der Kugel, so gehören $P_{1}$ und $P_{2}$ zur Eigenschattengrenze $s$ des Ringes. - Hieraus ergibt sich die folgende Konstruktion der Kurve $s^{\prime}$ : Wir beschreiben am $a^{\prime}$ mit dem Radius $r$ den Kreis $l^{\prime}$ als ersten scheiubaren Umrib der Hilfskugel, ermitteln wie früher den Aufrif. $l_{0}^{\prime \prime}$ des $\| \Pi_{2}$ gedrehten Lichtstrahls $l$ und ziehen in $m_{0}^{\prime \prime}$ den Radius $K_{0}^{\prime \prime} C_{0}^{\prime \prime} \perp l_{0}^{\prime \prime}$. Der Grundrib von \& ist bekanntlich eine Ellipse $\mathfrak{g}^{\prime}$ mit dem Mittelpunkt $a^{\prime}$ und der großen Halbachse $r \perp l^{\prime}$; die kleine Halbachise ist gleich dem Abstand des Punktes $C_{0}^{\prime \prime}$ von der Parallelen zu $a^{\prime \prime}$ durch $K_{0}^{\prime \prime}$ (Art. 87). Hachen wir dann auf einem beliebigen Durchnesser $P^{\prime} Q^{\prime}$ von $\xi^{\prime}$ die Strecken $P^{\prime} P_{1}^{\prime}=P^{\prime} P_{2}^{\prime}=Q^{\prime} Q_{1}^{\prime}=Q^{\prime} Q_{2}^{\prime}=d$, so sind $P_{1}^{\prime}, P_{2}^{\prime}, Q_{1}^{\prime}, Q_{2}^{\prime}$ vier Punkte von $s^{\prime}$. - Die Kurve $s$ besteht hier-

Fig. 142 .
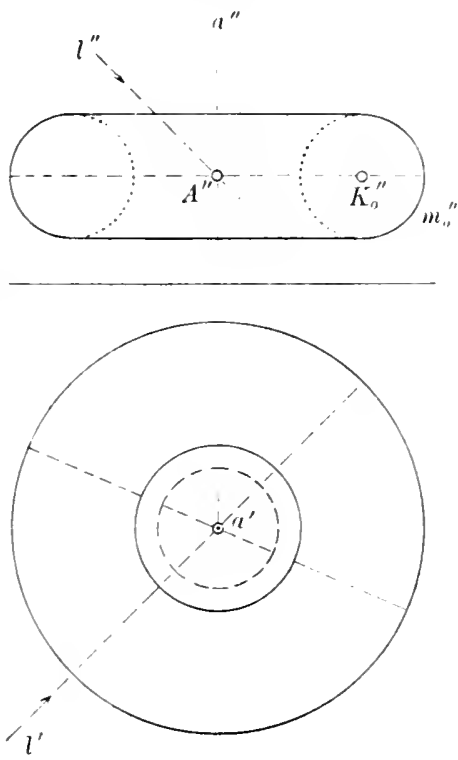
nach aus zwei Teilen, von denen der eine dem elliptischen, der andere dem hyperbolischen Teile der Ringfläche angehört.

Die Punktpaare $P_{1}, P_{2}$ und $Q_{1}, Q_{2}$ liegen in zwei Horizontalebenen, die von $A$ gleich weit entfernt sind. Wir finden ihre zweiten Projektionen mit Hilfe der zugehörigen Parallelkreise.

Auch zur Konstruktion des Schlagschattens, den der Ring auf die $\Pi_{1}$ wirft, benutzen wir die Hilfskugel um $A$. Ihre Schlagschattengrenze ist eine Ellipse $\xi_{h}$ mit dem Mittelpunkte $A_{h}$ und der kleinen Halbachse $r$; ziehen wir an $m_{0}^{\prime \prime}$ die Tangente $C_{0}^{\prime \prime} N_{0}^{\prime \prime} \| l_{0}^{\prime \prime}$ bis $A^{\prime \prime} K_{0}^{\prime \prime}$, so ist die große Halbachse $=K_{0}^{\prime \prime} N_{0}^{\prime \prime}$. Denken wir uns ron den Punkten $P$ und $P_{1}$ den Schlagschatten $P_{h}$ und $P_{1 h}$ ermittelt, so wird $P_{h} P_{1 h}$ \# $P^{\prime} P_{1}^{\prime}$, also $=d$. Nach Art. 146 ist $P_{h} P_{1 h}$ die Normale von $\varepsilon_{h}$ in $P_{h}$. Daraus folgt: Die Schlagschattengrenze $s_{h}$ des Ringes ist die Parallelkurre der Ellipse ${ }_{h}$ im Abstande $d$ (vgl. Art. 145).

Den Rückkehrpunkten von $s_{h}$ entsprechen auf dem hyperbolischen Teile der Ringfläch die Punkte von $s$. in denen die Kurve ron je einem 
Lichtstrahl berührt wird. Ist $l_{i}$ ein solcher Berührungspunkt auf der oheren Hälfte des Ringes, so beginnt in ihm der Schlagschatten $s_{*}$, den ein 'Teil von $s$ anf die Ringfläche wirft, und insofern ist $R$ ein Endpunkt der Eigenschattengrenze. Wir ermitteln $s_{*}$, indem wir von einem passend gewählten Parallelkreis, etwa dem Kehlkreis, den Schlagschatten auf die $\Pi_{1}$ konstruieren und seine Schnitpmukte mit $s_{h}$ aufsuchen. Die Kurven $s$ und $s_{*}$ berühren einander in $l$.

148. Konstruktion des Schlagschattens, den eine Hohlkehle von ihrem Randkreise empfängt. In Fig. 143 ist der

Fig. 143.

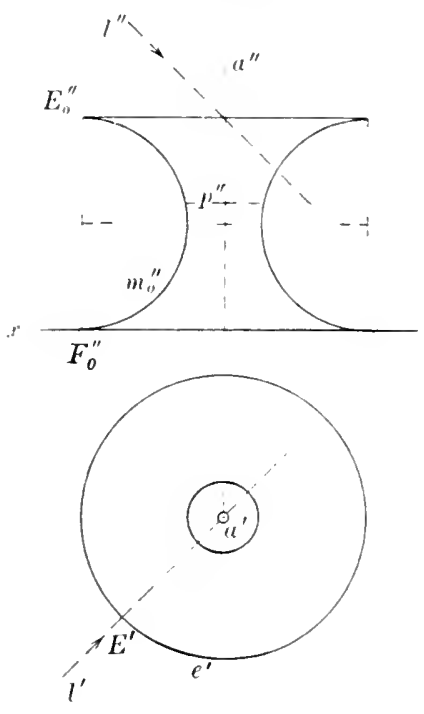

Meridian $m_{0}$ ein Halbkreis mit dem rertikalen Durchmesser $E_{0} F_{0}$. Der Schatten $e_{*}$, den der Parallelkreis $e$ des Punktes $E_{0}$ auf die Undrehungsfläch $\theta$ wirft, ist ein Teil der Durchdringungskurve der Fläche mit dem Lichtstrahlenzylinder, der $e$ zur Leitkurvo hat. Er ist symmetrisch in bezug auf die zu den Iichtstrahlen parallele Meridianebene $\mathrm{M}$; sein höchster Punkt ist also der Schatten $E_{*}$ des in $\mathbf{M}$ liegenden Punktes $E$ von $e\left(E_{0}^{\prime \prime} E_{* 0}^{\prime \prime} \| l_{0}^{\prime *}\right.$ bis mo usw.).

Um auf dem Parallelkreis $p$ die Punkte $I^{\prime}$ und $Q$ der lírve $e_{*}$ zu ermitteln, konstruieren wir den Schatten $e_{1}$ von $e$ auf die Ebene ron $p$; dann sind $P$ und $Q$ die Schnittpunkte vou p) mit $e_{1}$.

Der auf $m_{0}$ liegrende Punkt von $e_{*}$ ergibt sich als Schnittpunkt von $m_{0}$ mit dem elliptischen Schatten, den die Ebene des Umrißmeridians vom volderen Halbkreise $e$ empfängt.

In den Schnittpunkten von $e_{*}$ mit der Eigenschattengrenze der Fläche sind die Tangenten von $e_{*} \| l$ (Art. 125).

149. Sclıttenkonstruktion bei einem Säulenkapitell für Licht in der Richtung der Würfeldiagonale. Der in Fig. 144 dargestellte Körper besteht ans einem geraden Kreiszylinder mit der Achse $a$ und dem Grundkreis $k$, einem Wulst mit dem Viertelkreis $m_{0}$ als Meridiankurve und einer quadratischen Deckplatte. Wir konstruieren:

1. die Eigenschattengrenze e des Zylinders;

2. die Eigenschattengrenze $s$ des $W^{\prime}$ lstes - anch im Grundriß wie in Art. $1+6$, also insbesondere die Punkte ' $T$ ' auf dem gröBten Parallelkreis $u, W$ auf $m_{0}$, $Z$ auf dem Parallelkreis durch $W$ und den tiefsten Punkt $D$ in der zu den Lichtstralılen parallelen Meridianebene;

3. den Schlagschatten $s_{*}$ von $s$ auf den Zylinder, indem wir durch die gefundenen Punkte von $s$ Lichtstrahlen bis an den Zylinder ziehen. Uabei liefert der Punkt $D$ von $s$ den höchsten Punkt $D_{*}$ vou $s_{*}$. Der durch den Schnittpunkt $E_{*}$ von $s_{*}$ mit e gehende Lichtstrahl berührt $s_{*}$ 
und bestimmt auf $s$ den letzten Punkt $E$, der noch Schatten auf den Zylindel wirft.

4. Der elliptische Schatten der Kante $A B$ der Deckplatte auf dem $Z_{y}$ linder erscheint im Aufrib als Kreisbogen mit dem Radius von $l_{i} \mathrm{um}$ den Schnittpunkt $O^{\prime \prime}$ von $a^{\prime \prime}$ mit rem Aufrib des durch $A$ gehenden Lirhtstrahls (Art. 127). Der durch den Schnittpunkt $F_{*}$ dieses Schattens mit der Kurve $s_{*}$ nach rückwärts gezogene Lichtstrahl berührt den Wulst in einem Punkte $F$ von $s$ und schneidet $A B$ in einem Punkte $G$. Dann empfängt der Zylinder von dem Abschnitt $A G \operatorname{der}$ Kante $A B$ den Schatten $A_{*} F_{*}$ und rom Bogen $F E$ der Kurve $s$ den Schatten $F_{*} E_{*}$.

5. Der Scliatten der Kante $A B$ auf den Wulst beginnt in $F$ und ist ein Teil der Schnittkurve $c$ des Wulstes mit der Lichtstrablenebene durch $A B$. Um auf irgend einem Parallelkreise $p$ die Punkte vou $c$ zu ermittelu, könnten wir den Schatten von $A B$ auf die Ebene von $p$ konstruieren. Noch kürzer ist bei der gewählten Lichtrichtung das folgende Verfahren: Der Zylinder mit der Achse $a$ und dem Grundkreise $p$ empfängt von $A B$ einen elliptischen Schatten, der sich im Aufriß als Kreis um $O^{\prime \prime}$ mit dem Radius von $p$ projiziert. Die Schnittpunkte dieses Kreises mit der Geraden $\nu^{\prime \prime}$ liegen auf $c^{\prime \prime}$. - Schneidet $c^{\prime \prime}$ die Kurve $s^{\prime \prime}$ zum zweiten Mal in $H_{*}^{\prime \prime}$, so ist der Bogen $F H_{*}$ von $c$ der Schatten des Abschnitts $G H$ der Kante $A B$, und die durch $G$ und $H$ gehenden Lichtstrahlen berühren $c$ in

Fig. 144.

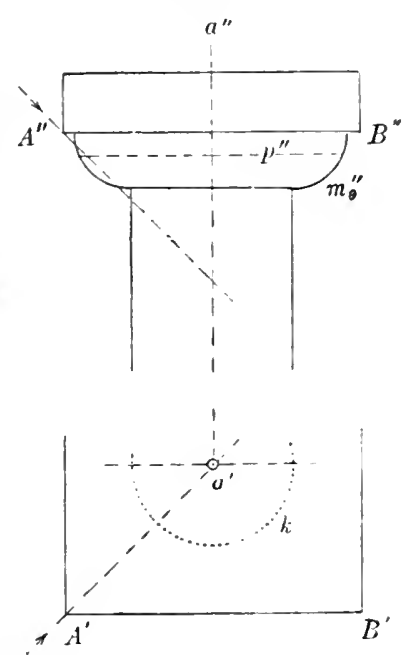
$F$ und $H_{*}$.

6. Der Schatten der $\perp \Pi_{2}$ durch $A$ gehenden Kante der Deckplatte auf den Wulst und auf den Zylinder fällt im Aufriß in die Gerade $A^{\prime \prime} A_{*}^{\prime \prime}$. Er begrenzt auf $s$ ein zweites Bogenstück, das auf den Zylinder Schatten wirft.

Die gesante Schattenkonstruktion kanu nach Art.12 \& I auch in der Weise ausgeführt werden, daß man den gegebenen Körper in der Lichtrichtung durch eine Reihe vertikaler Hilfsebeuen schneidet und für jede Schnitffigur die streifenden Lichtstrahlen zeichnet. Dieses Verfahren empfielilt sich jedoch nur, weun das Kapitell eine sehr verwickelte Form hat.

\section{Durchdringungen.}

150. Aufgabe. Die Durchdringung einer Cmdrehung:fläche, deren Achse $u \perp \Pi_{1}$ ist, mit einer Kegelfläche zu konstruieren, deren Leitkurve $k$ in $\Pi_{1}$ liegt (Fig. 1t5). Um für einen beliebigen Parallelkreis $p$ der Umdrehungsfläche seine Schnittpunkte mit dem Kegel zu ermitteln, legen wir durch die Spitze $S$ deKegels und durch $p$ eine Hilfskegelfläche und konstruieren ihreu S̈chnittkreis $p_{1}$ mit $\Pi_{1}$. Schneidet $p_{1}$ die Kurve $l_{i}$ in $P_{1}$ und $Q_{1}$, so 
haben die beiden konzentrischen Kegelflächen die Inatellinien S $Y_{1}^{\prime}$ und $S Q_{1}$ gemein, und diese treffen $p$ in zwei Punkten $l$ nnd $Q$ der Durchdringungskurve.

151. Aufgabe. Die Durchdringung zweier Tumdrelungsflächeu $A$ und B zu koustruieren, deren Achsen a und bsich

Fig. 145 .
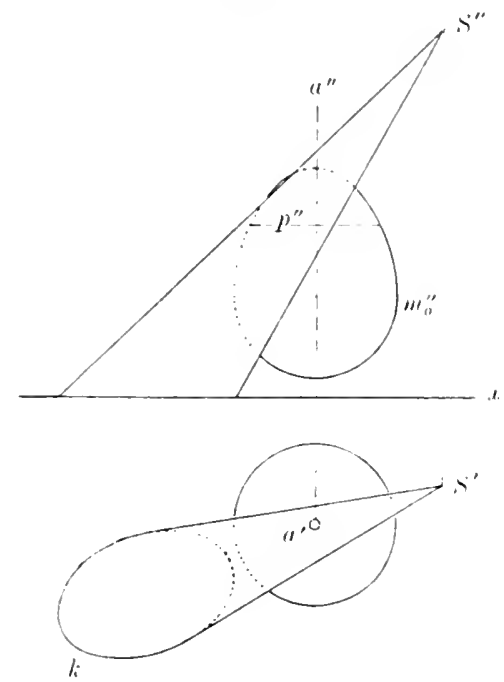

Fig. 146 .
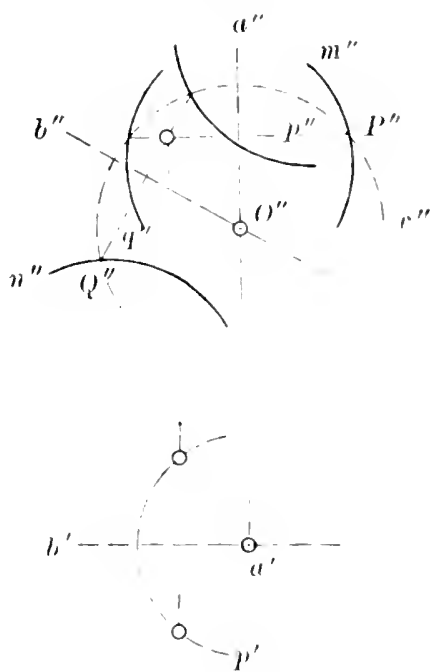

schneiden (Fig. 146). Sei $a \perp \Pi_{1}, b \| \Pi_{2}$; die Ebene $E=a b$ schneide $A$ und $B$ bzw. in den Umrißmeridianen $m$ und $n$. Zur Konstruktion

Fig. 147
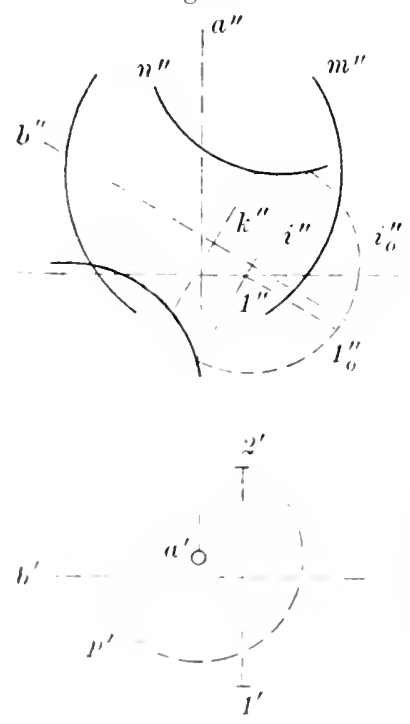
der Durchdringungskurve $c$ benutzen wir als Hilfsflächen nicht, wie gewöhnlich, Ebenen, sondern Kugelflächen um den Schnittpunkt $O$ von a und $b$; denn diese erzeugen sowohl mit $A$ als mit $B$ die einfachsten Sclmitte, nämlich Parallelkreise. Beschreiben wir als zweiten scheinbaren Umrils einel solchen Hilfskugel mit beliebigem Radius um $O^{\prime \prime}$ den Kreis $v^{\prime \prime}$, der die Kurven $m^{\prime \prime}$ und $n^{\prime \prime}$ bzw. in $\Gamma^{\prime \prime}$ und $Q^{\prime \prime}$ schneidet, so hat die Kingel mit $A$ und B bzw. die Parallelkreise $r$ und $q$ der Punkte $l$ ' und $Q$ gemein, und diese treffen sich im allgemeinen in zwei Punkten Ji und $S$ von $c$. Wir erhalten zunächst $R^{\prime \prime}=S^{\prime \prime}$ als Schnittpunkt der Strecken $l^{\prime \prime}$ und $q^{\prime \prime}$ und finden dann $I^{\prime}$ und $S^{\prime}$ auf dem Kieise $p^{\prime}$. - Die Kurve $c$ ist symmetrisch in bezug auf $E$.

Man löse in rieser Weise noch einmal die in Art. 11s hehandelte Aufgabe. 
152. Anfgabe. Die Durchdringung zweier Emarehungsflachen A und B mit windschiefen Achsen a und $b$ zu konstruieren (Fig. 147). Wir nehmen wieder $a \perp \Pi_{1}, b \| \Pi_{2}$ und bezeichnen bzw. mit $m$ und $n$ die Umrimeridiane beider Flächen. Unter Anwenlung lorizontaler Hilfsebenen gestaltet sich die Konstruktion der Durchdringungskurve $c$ folgendermaßen: Die Hilfsebene $\Sigma$ schneidet A in einem Parallelkreise $p$ und B in einer gewissen Kurve q; dann geht $c$ durch die Schnittpunkte von $p$ und $q$. I'm $q^{\prime}$ \%u ermitteln. zeichnen wir im Aufrib eine Reihe von Parallelkreisen $i, k \ldots$ der Fläche B und bestimmen für jeden von ihnen seine Schnittpunkte mit $\Sigma$. 'Trifft 2. B. ; die Ebene $\Sigma$ in 1 und 2, so fällt $2^{\prime \prime}$ mit $1^{\prime \prime}$ zusammen, und wir erhalten die Punkte im Grundrif durch Drehung des vorderen Halbkreises $i$ in die Lage $i_{0} \| \Pi_{2}$. Kommt hierdurch 1 nach $1_{0}$, so ist $1^{\prime \prime} 1_{0}^{\prime \prime} \| l^{\prime \prime}$, und dann sind die Abstände der Punkte $1^{\prime}$ und $2^{\prime}$ von $l^{\prime}$ $=1^{\prime \prime} 1_{0}^{\prime \prime}$.

\section{Schraubenflächon.}

Kurvenerzeugung durch Rollung.

153. In der Zeichenebene sei die feste Kurve $f$ und die bewegliche Kurve $w$ gegeben (Fig. 148). Dann sagen wir, die Kurve $w$ rollt auf $f$, wenn sie $f$ beständig berührt, und wenn der Berïhrungspunkt auf beiden Kurven um gleiche Bogenstücke fortschreitet. Betrachten wir beicle Kurven als Vielecke mit unendlich kleinen, entsrechend gleichen Seiten, so erkennen wir, daß der Übergang von $u$ ans einer Lage in die unendlich benachbarte als eine unendlich kleine Drehung um den augenblicklichen Berülırungspunkt $P$ aufgefaßt werden kann. Denken wir uns daher mit der Kurve $w$ einen Punkt $A$ ihrer Ebene starr verbunden, so bewegt sich dieser momentan senkrecht zur Geraden $A I$. Hieraus folgt der Satz: Wird die Bewegung einer Ebene erzeugt durch das Rollen einer Kurve w auf einer anderen Kurve $t^{\circ}$, so gebt für jeden Pnnkt der bewegten Ebene die Normale seiner Babnkurve in jerler Lage des Punktes durch den augenblicklichen Berührungspunkt von w und $f$. Der Berührungsfmnkt heißt der augenblickliche Pol der Bewegung.

Die Bahnkurve des Punktes $P$ von $w$ hat an der mit $P$ bezeichneten Berübrungsstelle im allgemeinen einen Rückkehrpunkt, dessen Normale mit der gemeinschaftlichen Tangente von $w$ und $f$ zusammenfällt. Dies ergibt sich sofort, wenn wir die Kurven $w$ und $f$ wie vorhin durch Vielecke ersetzen. Dabei wird aber angenommen, daf $I$ kein singulärer Punkt von " oder $f$ ist, und daf diese Kurven einander in I' nicht oskulieren.

154. Ist $f$ eine Gerade, $w$ ein Kreis, so beschreibt jeder Punkt der bewegten Ebene eine Zykloide, die als gespitzt (gemein), ver: 'hlungen oder geschweift (gestreckt) bezeichnet wird, je nachdem 
der beschreibende Punkt auf, außerhall oder innerhalb $u$ liegt. Sei $\mu_{0}$ die Anfangslage ron $w, M_{0}$ der Mittelpunkt, $P_{0}$ der Berührungspunkt ron $u_{0}$ mit $f$ (Fig. 149). Um den Zykloidenbogen zu konstruieren, den tler auf $M_{0} I_{0}^{\prime}$ liegende l'unkt $A_{0}$ erzengt, wenn $w$ anf $f$ eimmal

Fig. 149.

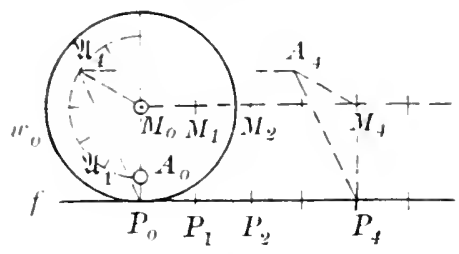

vollständig abrollt, machen wir anf $f^{*}$ die Strecke $I_{0} I_{n}$ gleich dem Umfang von $u$, beschreiben $1 \mathrm{~m} \boldsymbol{I}_{0}$ mit $M_{0} A_{0}$ einen Kreis a und teilen ihu von $A_{0}$ aus sowie $P_{0} P_{n}$ in $n-$ z. B. 12 - gleiche 'Teile. Sind $l_{i}$ und $M_{i}$ ein Paar entsprechender 'Teilpunkte, so erhalten wir die Lage $A_{i}$, in die der Punkt $A$ grelangt, weun $w$ die Gerade $f$ in $P_{i}$ berührt, indem wir clen Kreis $u_{0} n$ m $M_{0}$ drehen, bis $A_{0}$ nach $\mathfrak{P}_{i}$ komnt, und ihn daun $\| f$ um die Strecke $P_{0} l_{i}^{\prime}$ verschieben. Demmach ist $\mathscr{N}_{i} A_{i}+P_{0} P_{i}$.

Übertragen wir die auf $f$ liegende Teilung von $M_{0}$ ans anf die Parallele zu $f$ nach $M_{1}, M_{2} \ldots$, so ergibt sich $A_{i}$ auch aus der Bedingumg $M_{i} 1_{i} \# M_{0} \mathfrak{H}_{i}$.

Die Normale der Zykloide in $A_{i}$ geht durch den Berïhrungspunkt $P_{i}$ von $w_{i}$ mit $f$, ist also $\# P_{0} \mathcal{H}_{i}$. Man erhält daher die Zykloide noch einfacher (nnd zumeist mit hinreichender Genanigkeit) als Einhüllende der Kreise um $P_{1}, P_{2} \ldots$ mit den Radien $P_{0} \mathfrak{A}_{1}, l_{0}^{\prime} \mathfrak{P}_{2} \ldots$

155. Rollt umgekehrt die Crerade $w$ aus der Anfangslage $u_{0}$ auf dem Kreise $f$, so beschreibt jeder Punkt von $u$, z. B. der augenblickliche Berührungspunkt $A_{0}$, eine gespitzte (gemeine) Kreisevolvente (Fig. 150). Zu ihrer Konstruktion rektifizieren wir den Kreis

Fig. 150.

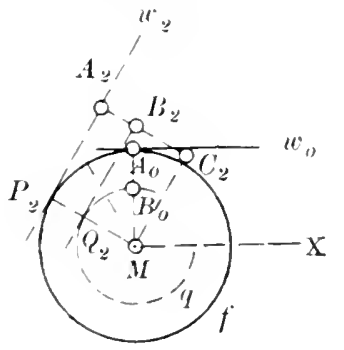
nnd teilen ihn von $A_{0}$ aus in $n$ gleiche Teile. Ziehen wir in Teilyunkte $I_{i}$ die Tangente $u_{i}$ und machen auf ibr die Strecke $P_{i} A_{i}={ }_{n}^{i}$ von $f$, so ist $A_{i}$ ein Pmukt der Evolvente.

Die Kreisevolvente ist eine Supirale; sio zieht sich in unendlich vielen Windungen um den Kreis f' und lat in $A_{0}$ einen Rückkehrpunkt, sowie mneudlich viele Doppelpunkte auf rer Verbindungslinie von $A_{0}$ mit dem Mittelpunkte $M$ von $f$.

Nach Art. 153 ist $A_{i} I^{\prime}$ ' die Normale der Evolvente in $A_{i}$; mithin ist $f$ die Erolute del Kurve und $P_{i}$ ihr hrümmungsmittelpunkt für den l’unkt $\mathbf{l}_{i}$.

Jeder I'unkt au Berhall, $w$ beschreibt in Verbindung mit der rollenden Tangente eine allgemeine Kreisevolvente, die wir als verschlungen oder groschweift bezeichnen, je naelidem dor betreffende I'unkt sich init dem Punkte $M$ auf derselben Seite, oder anf entgegengesetzten Sieiten ron $w$ befindet. Wählen wir den beselueibenden P'unk $B$ auf dem Jote in $A$ zu $w$, so ergibt sich seine Lage $\mathscr{S}_{i}$ mittels $A_{i} I_{i} \perp w_{i}$ und $=A_{0} B_{0}$. Schneidet $I H_{\imath}$ die l'arallele durch $B_{i}$ zu $u_{i}$ in $Q_{i}$, so 
berührt $Q_{i} B_{i}=P_{\imath} A_{i}$ den hreis $I$ um $M$ mit dem Radius $M B_{0}$ : wir erhalten daher die Bahukurve des Punktes $B$, indem wir auf den Tangenten vou $q$ in $Q_{1}, Q_{2} \ldots$ die Längen $\frac{1}{n} f, \frac{2}{n} f \ldots$ abtragen. Da die Kurveunormale in $B_{i}$ durch den Pol $P_{i}$ geht, so ist die Kurve die Einhüllende der Kreise um $P_{1}, P_{2} \ldots$ durch $B_{1}, B_{2} \ldots$

Sei $C$ der Punkt der bewegten Geraden $A B$, dessen Anfangslage $C_{0}$ mit $M$ zusammenfällt, und $C_{i}$ die Lage, die er einnimmt, wenn $u$. nach $u_{i}$ gelangt. Ziehen wir die Gerade $M X \| u_{0}$ und setzen $M A_{0}=a$ und $-X M C_{i}=\varphi$, so ist auch $-A_{0} M P_{i}=\varphi$, mithin $M C_{i} \# I_{i} A_{i}$ $=a \varphi$; die Bahnkurve des Punktes $C$ entsteht also, wem der Strahl $I X$ um $M$ gedreht wird, und wenn gleicbzeitig $\operatorname{der}$ Punkt $C$ von der Anfangslage $M$ aus wich auf $M X$ verschiebt, so daß gleichen Drehungswinkeln gleiche Verschiebungsstrecken entsprechell. Die so erzengte Kurre heißt eine archimedische sipirale.

\section{Die Schraubenlinie.}

156. Eine Schraubenlinie entsteht, wenn ein Punkt sich un eine feste Achsedreht und zugleich proportional zu dieser Drehung parallel zur Achse verschiebt, so daß also gleichen Drehungswinkeln gleiche Verschiebungsstrecken entsprechen. Eine solche Bewegung heißt Šchraubung. Wir bezeichnen die feste Achse als Schraubenachse, die Entfernung des bewegten Punktes von der Achse als Radius der Schraubenlinie, den geraden Kreiszylinder, auf dem sich die Kurve befindet, als Schraubenzylinder. Unter Ganglı̈̈be versteht man die einer vollen Umdrehung entsprechende Größe der Verschiebung, also die konstante Entfernung zweier aufeinanderfolgenden Schnittpunkte irgend einer Mantellinie des Zylinders mit der Kurve. Die Schraubenlinie ist rechts gewunden. wenn sie für einen in die Achse (gleichgültig ob aufrecht oder verkehrt) gestellten Beschauer nach rechts abwärts geht.

Fig. 151 zeigt eine rechts gewundene Schraubenlinie $b$ mit vertikaler Achse $a$ in schiefer Parallelprojektion: die Anfangslage $B_{0}$ des beschreibenden Punktes befindet sich auf dem Grundkreise $k$ des Schraubenzylinders. Schneiden wir den Zylinler in der durch $B_{0}$ gehenden Hantellinie auf und wickeln ihn in die Zeichenebene ab, so verwandelt sich $l$ in eine Gerade und $b$ in eine Kurve, bei der sich die mit den Mantellinien zusammenfallenden Ordinaten der Punkte $B_{1}, B_{2} \ldots$ wie lie auf $l$ von $B_{0}$ aus gemessenen Abszissen verbalten (Fig. 15la). Die Verwandelte der Schraubenlinie ist also eine Gerade, und umgekehrt entsteht durch Aufwickelung einer Ebene auf einen geraden Kreiszylinder aus jerler Gerarlen der Ebene eine Schraubenlinie. Die Schraubenlinie ist daher die kürzeste (geodätische) Linie auf lem geraden Kreiszylinder.

Da die Gerade $b$ mit deu abgewickelten Mantellinien gleiche Winkel einschliebt, so schneidet auch die Schraubenlinie alle Ilantellinien des Zylinders unter demselben Winkel. Demuach bildeu die Tangenten in allen Punkten der Kurve mit der Ebene des Grund- 
kreises $k$ einen koustanten Neigungswinkel $\boldsymbol{\beta}$, der den vorher genannten Winkel zn einem Rechten ergänzt. Wir nennen $\beta$ den Steigung:winkel der Schraubenlinie.

Für $\beta=0$ wird die Schraubenlinie zu einem Kreis, für $\beta=90^{\circ}$ zu einer Geraden.

Ist $\mathfrak{r}$ der Radius, $\mathfrak{G}$ die Ganghöhe der Schraubenlinie $b$, so ergibt sich aus Fig. $151 \mathrm{a}$

$$
\mathfrak{h}=2 \pi \mathfrak{r} \operatorname{tg} \beta
$$

Bezeichnen wir mit $B_{1} B_{2}$ and $B_{3} B_{4}$ zwei gleiche Bogenstücke ron b. mit $C$ und $D$ bzw. die Punkte, in denen die anf dem Schraubenzylinder durch $B_{1}$ and $B_{3}$ gelegten Kreise die Mantellinien durch $B_{2}$

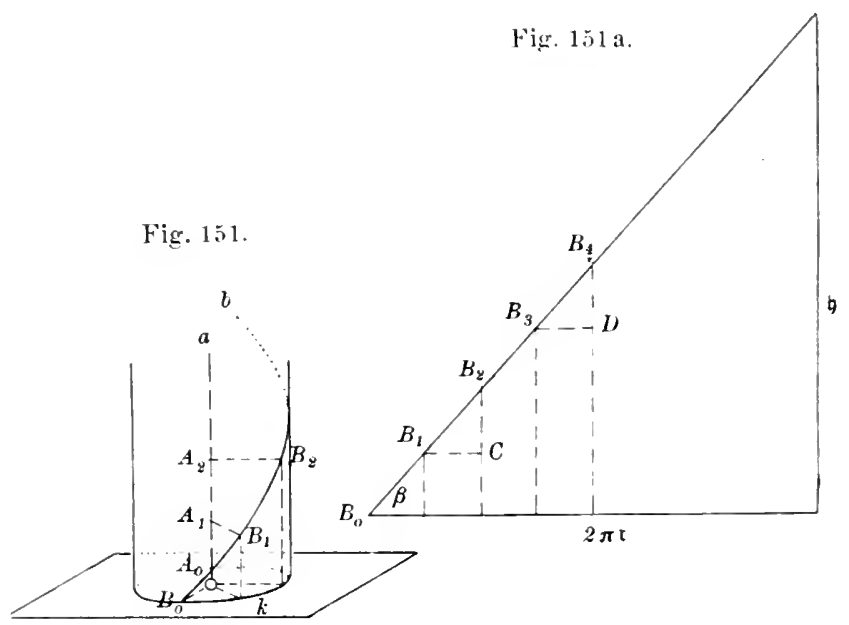

und $B_{4}$ schneiden. so haben die krummflächigen rechtwinkligen Oreiecke $B_{1} C B_{2}$ und $B_{3} D B_{4}$ paarweise gleiche Katheten, wir können also das eine durch Drehung nm $a$ und Schiebung \| $a$ mit dem anderen zur Deckung bringen. Daraus folgt: Gleiclue Bogenstïcke derselben Schraubeulinie sind kongruent. Die Schraubenlinie ist daher in sich selbst verschiebbar, d. h. sie hat in allen Punkten lenselben Krïmmugsradius.

157. Tm für irgend eiuen Punkt der Schraubenlinie $b$ den K r ümmungskreis der Größe und Lage nach zu bestimmen, ermitteln wir zanächst die Schmiegungsebene des Punktes. Dazn dient uns der Satz, daf die Schraubenlinie die kürzeste Verbinlung zweier Punkto auf dem gerarlen Kreiszylinder bildet.

Auf jeder krummen Fläche ist die kürzeste Linie die Gleichgewichtsform eines über die Fläche straff grespannten Fadens. Nun wirken in jedem Punkte des Fadens zwei Kräte in den Richtungen nach den beiderseits unendlich benachbarten Punkten, und ibre Mittelkraft, die nach der Regel des Krafteparallelogramms konstruiert wird, nuß in die Flächennormale fallen, damit der Faden in Ruhe bleibt. 
Tas Kräfteparallelogramm liegt aber in der Sichmiegungsebene des betrachteten Kurvenpuntes, mithin ergibt sich ler Satz: Die Schmiegnngsebene in irgend einem Punkte einer geodätischen Linie geht durch die zugehörige Flächennormale.

In einem Punkte $B$ der Sclirambenlinie $b$ ist diese Normale der liadius $B$, l, d. h. das Lot von $B$ auf die Achse $A$; die Schmiegungsebene $\Sigma$ geht daher durch $B A$ und die Tangente $t$ der Schraubenlinie in $B$. Der gesuchte Krümmungskreis liegt in der Ebene $\Sigma$, und da er $t$ in $B$ berührt, so befindet sicb sein Mittelpunkt $M$ auf der Geraden BA. - Wir ermitteln jetzt die Länge $\rho$ des Krümmungsradius $B M$ : Die Ebene $\Sigma$ schneidet den Schraubenzylinder in einer Ellipse mit dem Mittelpunkt $A$ und der kleinen Halbachse $A B=\mathfrak{r}$; die große Halbachse ist $\| t$ und $=\frac{\mathfrak{r}}{\cos \beta}$. Diese Ellipse hat mit der Schraubenlinie in $P$ drei unendlich nahe Punkte, also den Krümmungradius $Q$ gemein, folglich ist nach Art. 77

$$
\varrho=\left(\begin{array}{c}
\mathfrak{r} \\
\cos \beta
\end{array}\right)^{2}: \mathfrak{r}=\begin{gathered}
\mathfrak{r} \\
\cos ^{2} \beta
\end{gathered}
$$

158. Darstellung einer Schraubenlinie mit vertikaler Achse $a$ in Grund- und Aufriß (Fig. 152). Von der rechts gewundenen Schraubenlinie $b$ ist die Anfangslage $B_{0}$ des beschreibenden Punktes $I$ in $\Pi_{1}$ so grewählt worden, daß ihre Verbindungslinie mit dem Fubpunkte $A_{0}$ von $a$ anf $x$ senkrecht steht: außerdem ist noch die Ganghöhe $\mathfrak{h}$ gegeben. Im einen Gang von $b$ zu konstruiereu, teilen wir den Grundkreis $k$ des Schraubenzylinders in den Punkten $B_{0}, B_{1}^{\prime}$, $B_{2}^{\prime} \ldots$ in eine beliebige Anzahl, etwa acht, gleicher Teile, machen auf $a$ die Abschnitte $A_{0} A_{1}=A_{1} A_{2}=\cdots$ $={ }_{8}^{\mathfrak{h}}$ und zeichnen im Aufriß die horizontalen Strecken $A_{1} B_{1}, A_{2} B_{2} \ldots$ Verstehen wir unter $B_{i}$ irgend einen Punkt von $b$ und setzen $B_{0}^{\prime \prime} A_{i}^{\prime \prime}$ $=\mathfrak{x}, A_{i}^{\prime \prime} B_{i}^{\prime \prime}=\mathfrak{y},-B_{0} A_{0} B_{i}^{\prime}=\varphi$ und wie vorhin den Radius von $b=r$, so wird $\mathfrak{l}=r \sin \varphi$, und es verhält sich

also folgt

$$
\varphi: 2 \pi=\mathfrak{x}: \mathfrak{h},
$$
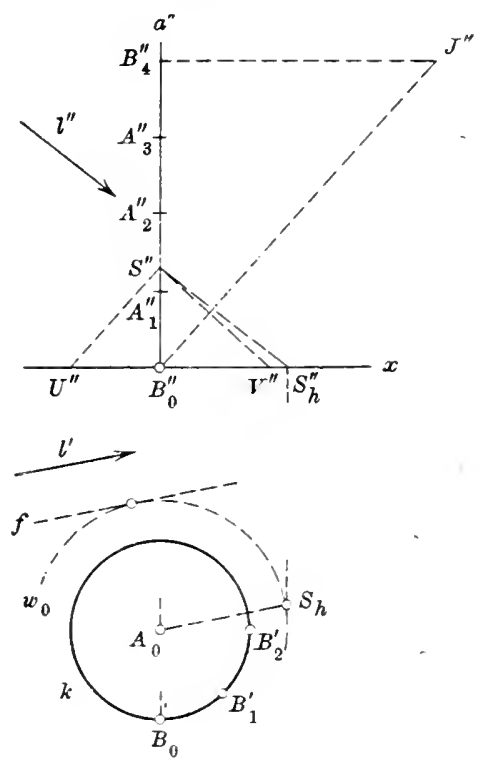

$$
\mathfrak{y}=\mathfrak{l} \sin ^{2 \pi} \mathfrak{h}:
$$

d. b: Ier Aufrib der Schraubenlinie ist eine Sinuskurve. 
Der Punkt $B_{0}^{\prime \prime}$ ist ein Wendepunkt der Kurve $b^{\prime \prime}$, demn die schmiegungsebene in $B_{0}$ ist $\perp \Pi_{2}$, weil sie durch $A_{0} B_{0}$ geht. Die Tangente $t_{0}$ von $b$ in $B_{0}$ liegt $\| \Pi_{2}$, ihr Aufrib bildet also mit $x$ den Steigungswinkel $\beta$, und dieser ergibt sich nach Fig. 151 a aus einem rechtwinkligen Dreieck, dessen Katheten sich wie $\mathfrak{h}: 2 \pi \mathfrak{r}$ verhalten. Ziehen wir daher die Gerade $B_{4}^{\prime \prime} J^{\prime \prime} \| x$ und machen sie $=\pi \mathrm{r}$, so ist $t_{0}^{\prime \prime}$ die Gerade $B_{0}^{\prime \prime} J^{\prime \prime}$.

Um die Tangente $t_{i}$ von $b$ im Punkte $B_{i}$ zn ermitteln, denken wir uns das auf dem Schraubenzylinder liegende Flächenstück $B_{0} b_{i} B_{i}^{\prime}$ in die durch $B_{i} B_{i}^{\prime}$ gehende Berührungsebene abgewickelt. Dann verwandeln sich die Bögen $B_{0} B_{i}^{\prime}$ und $B_{0} B_{i}$ bzw. in die Tangenten des Grundkreises in $B_{i}^{\prime}$ und der Schraubenlinie in $B_{i}$. Machen wir daher auf der Tangente von $l_{i}$ in $B_{i}^{\prime}$ die Strecke $B_{i}^{\prime} T_{i}$ gleich dem Kreisbogen $B_{i}^{\prime} B_{0}$, so ist $T_{i}$ die Grundrißspur ron $t_{i}$ und $t_{i}^{\prime \prime}=B_{i}^{\prime \prime} T_{i}^{\prime \prime}$. - Zu einer anderen Lösung gelangen wir in folgender Weise: Ziehen wir durch eineu beliebigen Puukt Parallelen zu allen 'Tangenteu von $b$, so entsteht ein gerader Kreiskegel mit zu a paralleler Achse und dem Basiswinkel $\beta$, der Richtungskegel der Schraubenlinie. Konstruieren wir diesen Kegel über dem Grundkreise $l$, so liegt seine Spitze $S$ auf $a$; um $S^{\prime \prime}$ zu erhalten, machen wir auf $x$ die Strecke $B_{0}^{\prime \prime} U^{\prime \prime}=\mathfrak{r}$ und zieheu $U^{\prime \prime} S^{\prime \prime} \| B_{0}^{\prime \prime} J^{\prime \prime}$. Nun kenuen wir vou der Tangente $t_{i}$ bereits den Grundrif $t_{i}^{\prime}$, und daraus ergibt sich auf dem Richtungskegel sofort die zu $t_{i}$ parallele Mantellinie $S R$; ihr Grundrib $A_{0} R$ ist nämlich $\perp A_{0} B_{i}^{\prime}$. Dann ist aber $t_{i}^{\prime \prime} \| S^{\prime \prime} R^{\prime \prime}$.

Die Höhe $A_{0} S$ des Richtungskegels ist $=r \operatorname{tg} \beta$, oder nach Gleichung 1) in Art. $156=\frac{\mathfrak{h}}{2 \pi}$. Wir setzen

$$
\frac{\mathfrak{h}}{2 \pi}=\mathfrak{h}_{0}
$$

und bezeichnen diese Strecke, also die der Drehung 1 entsprechende Schiebung, als reduzierte Ganghöhe.

Ziehen wir $S^{\prime \prime} V^{\prime \prime} \perp U^{\prime \prime} S^{\prime \prime}$ bis $x$, so wird $U^{\prime \prime} V^{\prime \prime}=\stackrel{\mathfrak{r}}{\cos ^{2} \beta}$, also nach Gleichung 2) gleich dem Krümmungsradius $\varrho$ der Schraubeulinie $b$. - Bestimmen wir anf clen Geraden $B_{0} A_{0}, B_{1} A_{1} \ldots z u$ den Punkten $B_{0}, B_{1} \ldots$ von $b$ die Krümmungsmittelpunlite $M_{0}, M_{1} \ldots$, so erhalten wir als Ort der Krümmungsmittelpunkte eine zweite Schraubenlinie $m$ von derselben Achse a und derselhen Ganghöhe $\mathfrak{h}$. Nach unserer Konstruktion von $\boldsymbol{g}$ ist $\mathfrak{h}_{0}$ die nittlere Proportionale der Radien dieser beiden Schraubenlinien, folglich ist $B_{i}$ auch der Kriummungsmittelpunkt von $m$ an cler Stelle $\boldsymbol{M}_{i}$, d. h. die Krïmmungsuittelpunkte von m liegen umgekehrtauf $b$.

Wir ermitteln schließlich den Krïmmungsradius der Sinuskurve $b^{\prime \prime}$ in den Scheiteln $K_{2}^{\prime \prime}, B_{6}^{\prime \prime} \ldots$ Der Krïmmungskreis der Schrambenlinie $b$ in $B_{2}$ erscheint im Aufrib als Ellipse mit der großen Halbachse $M_{2}^{\prime \prime} \boldsymbol{B}_{2}^{\prime \prime}=9$ und der kleinen Halbachse 9 sin $\beta$. l)iese Ellipse hat mit der Kurve $b^{\prime \prime}$ in $l_{3,2}^{\prime \prime}$ drei unendlich nahe Punkte gemein, mithin ist der gesuchte hrümmmugsradius voll $b^{\prime \prime}=(0 \sin \beta)^{2}: 0=r g^{2} \beta$ $=B_{i}^{\prime \prime} \Gamma^{\prime \prime}$. 
159. Schlagschatten der Schraubenlinie auf $\Pi_{1}$. Konstruieren wir in Fig. 152 bei gegebener Lichtrichtung $l$ vom Endpunkte $A_{\mathrm{S}}$ der Ganghöhe $A_{0} A_{8}$ den Schlagschatten $A_{8}^{h}$, so finden wir den Schatten von $B_{i}$, indem wir die Strecke $A_{0} A_{8}^{h}$ in acht gleiche Teile teilen und durch den Teilpunkt $A_{i}^{h}$ die Strecke $A_{i}^{h} B_{i}^{h} \# A_{i} B_{i}$, d. h. $\# A_{0} B_{i}^{\prime}$ ziehen. Dies ist aber die Konstruktion einer Zykloide, die der Punkt $B_{0}$ beschreibt, wenn der Kreis $w_{0}$, dessen Mittelpunkt $A_{0}$ und dessen Umfang $=A_{0} A_{8}^{h}$ ist, auf der zu $l^{\prime}$ parallelen Geraden $f$ rollt. Bezeichnet $\lambda$ den Neigungswinkel der Lichtstrahlen gegen $\Pi_{1}$, so ist $A_{0} A_{8}^{h}=\mathfrak{h} \cot \lambda$, also der Radius von $w_{0}=\frac{\mathfrak{h}}{2} \cot \lambda$, d. h. gleich dem Schatten $A_{0} S_{h}$ der reduzierten Ganghöhe.

Wir erhalten eine verschlungene, gespitzte oder geschweifte Zykloide, je nachdem $A_{0} B_{0} \gtreqless A_{0} S_{h}$ ist. Setzen wir hier für $A_{0} B_{0}$ aus Gleichung 1) den Ausdruck $\frac{\mathfrak{h}}{2 \pi t g \beta}$ und für $A_{0} S_{h}$ den oben gefundenen Wert, so geht diese Bedingung über in $\operatorname{tg} \lambda \gtreqless \operatorname{tg} \beta$. Daher der Satz: Der Schlagschatten der Schraubenlinie auf eine zu ihrer Achse senkrechten Ebene ist eine Zykloide, und zwar eine verschlungene, gespitzte oder geschweifte, je nachdem der Neigungswinkel der Lichtstrahlen gegen die Ebene größer ist als der Steignngswinkel der Schraubenlinie, oder ihm gleich oder kleiner ist als dieser. - Der Schlagschatten auf jede andere Ebene ist demnach eine affine Kurve zu einer Zykloide.

\section{Die abwickelbare Schraubenfläche.}

180. Die Tangenten der in Fig. 152 dargestellten Schraubenlinie b bilden eine abwickelbare Schraubenfläche, die von den Schmiegungsebenen der Kurve berührt wird; die Schraubenlinie ist die $\mathrm{R} u ̈ c k-$ kehrkante der Fläche (vgl. Art. 88). Diese Tangentenfläche schneidet die $\Pi_{1}$ in einer Evolvente $e$ des Grundkreises $k$ des Schraubenzylinders mit einem Rückkehrpunkte in $B_{0}$. Der eine Zweig von $e$ enthält die Grundrißspuren der 'Tangenten der oberhalb $\Pi_{1}$ liegenden Punkte von $b$; der andere Zweig entspricht dem Teile von $b$, der sich unterhalb $\Pi_{1}$ befindet.

Da die Tangente $t_{i}$ des Punktes $B_{i}$ von $b$ auf dem Radius $B_{i} A_{i}$ senkrecht steht, so ist sie eine Fallinie der Schmiegungsebene der Schraubenlinie in $B_{i}$, d. h. der Berührungsebene der abwickelbaren Schraubenfläche. Diese Ebene bildet mit $\Pi_{1}$ den konstanten Neigungswinkel $\beta$; deshalb bezeichnet man die abwickelbare Schraubenfläche als eine Fläche ron gleichförmiger Neigung.

Die Inflexionstangenten der Sinuskurve $b^{\prime \prime}$ bilden den zweiten scheinbaren Umriß der Fläche, weil die entsprechenden Berührungsebenen auf $\Pi_{2}$ senkrecht stehen.

Jede horizontale Ebene schneidet die Fläche in einer zu $e$ kongruenten Kurve, die wir aus $e$ durch Schraubung um $a$ erhalten. Bei 
dieser Schraubung beschreibt jeder Moppelpunkt von " eine Schraubenlinie, in der die Fläche sich selbst durchschneidet, also eine loppelkurve der Fläche.

161. Die Eigenschattengrenze der Fläche. Bei jeder abwickelbaren Fläche besteht die Eigenschattengrenze aus Mantellinien; demn ist $I^{\prime}$ ein Punkt der Eigenschattengrenze und $t$ die durch ihn gehende Mantellinie, so berïhrt die durch $t$ in der Lichtrichtung selegte Fbene die Fläche nicht nur in $I$, sondern in allen Punkten von 1.

Im die Eigenschattengrenze der Tangentenfläche der Schraubenlinie $b$ zu ermitteln, benutzen wir den in Fig. 152 konstruierten Richtungskegel, der $k$ zum Grundkreis und $S$ zur Spitze hat. Denken wir uns zu zwei unendlich benachbarten Tangenten von $b$ die parallelen Mantellinien des lichtungskegels gezeichnet, so sind auch die Ebenen parallel, die durch diese Geraden bestimmt werden; die abwickelbar $\mathrm{A}$ Schraubenfläche und ihr Richtungskegel haben also in parallelen Mantellinien parallele Berührungsebenen. Daraus folgt, daß die Eigenschattengrenzen beider Flächen einander parallel sind. In Fig. 152 gehen nun die Eigenschattengrenzen des hegels nach den Berührungspunkten $F$ und $G$ der Tangenten aus $S_{h}$ an 1. : wir erhalten daher im Grundriß die Eigenschattengrenze der Schraubenfläche, indem wir $\| A_{0} F$ und $A_{0}$ ( die Tangenten von $k$ ziehen, denen Tangenten von $b$ entsprechen, die zn $S F$ und $S G$ parallel sind.

182. Bei der Abwickelung der Schraubenfläche ändert sich weder das Bogenelement $d s$ der Rückkehrkante $b$, noch der Winkel $d q$ zwischen zwei aufeinander folgenden Elementen von b, also auch nicht ler Krümmungsradius $\varrho=\frac{d s}{d \vartheta}$. Die Rückkehrkante verwaudelt sich demnach in einen Kreisbogen rom Radius o. Für einen vollen Schraubengang ist die Bogenlänge $B_{0} B_{8}$ gleich der Hylotenuse eines rechtwinkligen Dreiecks mit den Katheten $2 \pi \mathfrak{r}$ und $\mathfrak{h}$, oder - 2. $B_{0}^{\prime \prime} J^{\prime \prime}$ in Fig. 152. Die spurevolvente $e$ verwandelt sich in die Erolvente des Kreisbogens.

\section{Die Schraubenflächen im allgemeinen.}

183. Eine Schraubenfläche entsteht durch Schraubung einer unveränderlichen Kurve nmeine Achse. Dabei beschreiben alle Punkte der Kurve Schraubenlinien ron derselben Achse und gleicher Ganghöhe. Jede Schraubenfläche ist dalıer in sich sellost verschiebbar. - Als Erzengende kann jele kiurve betrachtet werden, die alle Schraubenlinien der Fläche schneidet. The ebenen Srhnitte der Fläche, die die Achse enthalten, heißen Meridiankurven (Profilkurven); diejenigen, die anf der Achse senkrecht stehen, werden als Normalkurven (Basiskurven) bezeichnet. Die Meridianknrven sowohl, wie die Normalkurven gehen durch Schraubung ineinander über, sind also unter siclı kongruent.

Ist you einer Schraubenfliche die Aclise $a \perp \Pi_{1}$, die Erzeugende $\cdot\left(c^{\prime}, c^{\prime \prime}\right)$, sowie die (ianghïhe h und der Sim der Schranbung gegelıen, $s$ erhalten wir die Meridiankurve $m_{0}$ in der Ebene $M_{0} \| \Pi_{2}$, indem wir 
verschiedene Punkte $I^{\prime}, Q \ldots$ von $c$ der Reihe nach geschraubt denken. bis sie in die Ebene $M_{0}$ gelangen (Fig. 15:3). Bezeichnen wir mit $I_{0}$ die neue Lage von $I^{\prime}$, so ist $a^{\prime} P_{0}^{\prime} \| x$ und $=a^{\prime} P^{\prime}$, und die Entfernung des Punktes $P_{0}^{\prime \prime}$ von der durch $P^{\prime \prime}$ gehenden Parallele zu $x$ verhält sich zu h wie $\angle P^{\prime} a^{\prime} P_{0}^{\prime}$ zu $360^{\circ}$. Wir werden daher die Punkte $I^{\prime}, Q^{\prime} \ldots$ zweckmäßig so wählen, daß die Winkel zwischen der Parallele durch $a^{\prime}$ zu $x$ und den Geraden $a^{\prime} I^{\prime}$, $a^{\prime}\left(l^{\prime} \ldots\right.$ einfache Bruchteile von $360^{\circ}$ betragen. - Um die in der Ebene $\mathrm{N}$ liegende Tormalkurve $n$ zu ermitteln, bestimmen wir von den Schraubenlinien der Punkte $I^{\prime}, Q \ldots$ ihre Schuittpunkte $I_{1}^{\prime}, Q_{1} \ldots$ mit N. Dann ist $a^{\prime} P_{1}^{\prime}=a^{\prime} P^{\prime}$, und der Winkel $P_{1}^{\prime} a^{\prime} P^{\prime}$ verhält sich zu $360^{\circ}$ wie der Abstand des Punktes $I^{\prime \prime}$ von $N^{\prime \prime} z u$ h. Bei dieser Konstruktion ist es also vorteilhaft, wenn die Entferuungen der Punkte $Y^{\prime \prime}, Q^{\prime \prime}$.. von der Geraden $N^{\prime \prime}$ zur Gaughöhe $\mathfrak{h}$ in einem einfachen Verhältnis stehen.

Der erste wahre Umriß der Fläche wird von den Schraubenlinien derjenigen Punkte ron $\boldsymbol{m}_{0}$ gebildet, deren Tangenten $\| a$ sind. Der zweite Umrib ist der Ort solcher Punkte der geschraubten Normalkurve, deren Tangenten auf $\Pi_{2}$ senkrecht stehen. Ist die Normalkurve z. B. ein Kreis wie bei der gewundenen Säule des BarockFig. 153.

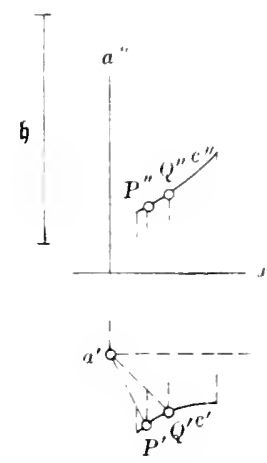
stils - so besteht der zweite scheinbare Umriß aus zwei Sinuskurven, die zum Aufriß der Schraubenlinie kongruent sind, die der Mittelpunkt des Kreises beschreibt.

Die Berührungsebene $\mathrm{T}$ in einem Punkte $B$ der śchraubenfläche ist bestimmt durch die Tangente an die durch $B$ gehende Erzeugende, sowie durch die Tangente an die Schraubenlinie b, die der Punkt $B$ bei der Entstehung der Fläche beschreibt. Durch Schraubung von T erhalten wir eine abwickelbare Schraubenfläche, welche die gegebene Fläche in allen Punkten von $b$ berührt. Sie gestattet dieselbe konstruktive Verwendung wie die Parallelkreisberührungskegel einer Umdrehungsfläche.

164. Eine Regelschraubenfläche entsteht durch Schraubung einer Geradell. Der Punkt der Erzeugenden, der der Achse am nächsten liegt, beschreibt die Kehlschraubenlinie. Die Fläche ist abwickelbar, wenn die Erzeugende die Kehlschraubenlinie berührt; andernfalls ist sie windschief. Bezeichnen wir mit $\gamma$ den Neigungswinkel der Erzeugenden gegen eine zur Achse senkrechte Ebene, mit $r$ ihren kürzesten Abstand ron der Achse, mit $\mathfrak{h}$ die Ganghöhe der Schraubung, so besteht im Falle einer abwickelbaren Schraubenfläche die Beziehung

$$
\mathfrak{h}=2 \pi \mathrm{r} \operatorname{tg} \gamma \text {. }
$$

Eine Regelschraubenfläche heißt gerade (normal) oder schief, je nachdem die Erzeugende rechtwinklig oder schief gegen die Achse gerichtet ist, und sie heißt geschlossen (axial) oder offen, je nachdem die Erzeugende die Achse schneidet oder nicht schneidet. Die gerade geschlossene Regelschraubenfläche wird auch als Wendelfläche bezeichnet. In Fig. 151 bilden die Geraden $A_{0} B_{0} . A_{1} B_{1} \ldots$ eine solche 
Wendelfäche, und dasselbe gilt von den oberen Kanten der Stufen bei einer Wendeltreppe.

165. Die Normalkurveder schiefon liegelschraubenfläche (Fig. 154). Wir gelıen von der abwickelbaren Schraubenfläche aus, die durch Schraubung der Geraden $t$ un die vertikale Achse $a$ entsteht. Die Strecke $A B$, die den kürzesten Abstand der beiden Geraden darstellt, bestimmt den Beriihrungspunkt $B$ von $t$ mit der Rückkehrkante $l$. Sei ferner $T$ der Schnittpunkt von $t$ mit $\Pi_{1}, k$ der Grundkreis ron $b$,

Fig. 154.

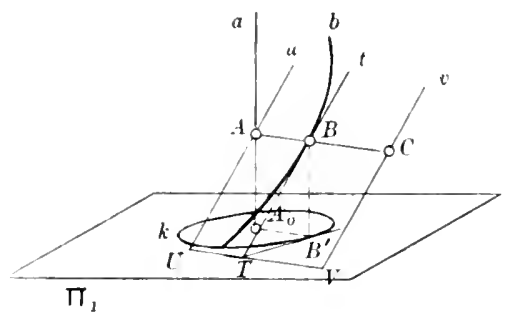
$\operatorname{der} A B$ zum Radius hat; dann berührt der Grundriß $B^{\prime} T$ von $t$ den Kreis $k$ in $B^{\prime}$. Ziehen wir noch durch $A$ und durch einen beliebigen Punkt $C$ vou $A B$ die Geraden $u$ und $v \| t$ und bezeichnen ihre Grundrißspuren mit $U$ und $V$, so ist $U T V \# A B C$ und $\perp B^{\prime} T$.

Wir lassen nun $u$ und $v$ an der von $t$ ausgeführten Schraubung teilnehmen. Dann erzeugt $u$ eine schiefe geschlossene, $v$ eine schiefe offene Regelschraubenfläche, und wir erhalten die in $\Pi_{1}$ liegenden Normalkurven aller drei Flächen als die Bahnen, wełche die Punkte $T, C, V$ in Verbindung mit der auf $k$ rollenden Tangente $B^{\prime} T$ beschreiben.

Fig. 155.
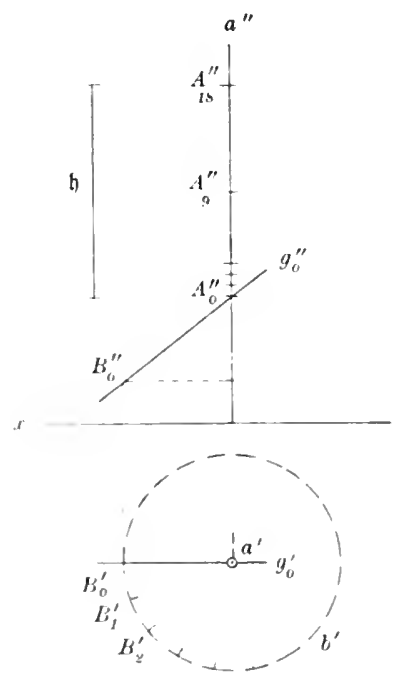
Daraus folgt nach Art. 155: Die Normalkurve der schiefen offenen Regelschraubenfläche ist eine allgemeine Kreisevolvente, diejenige der schiefen geschlossenen eine archimedische Spirale.

168. Darstellung einer schiefen geschlossenen Regelsehraubenfläche in Grund-und A u friß(Fig.155). Ton der rechts gewundenen Fläche sei gegeben die vertikale Achse $a$, die unbegrenzte Erzengende $g, \operatorname{die} a$ in $A$ schneiclet, in der Anfangslage $g_{0} \| \Pi_{2}$ und die Ganghöhe h. Um eine Anzahl von Lagen $y_{1}, y_{2} \ldots$ der Geraden ! $\mathrm{zu}$ zeichnen, konstruieren wir die Schraubenlinie b, die irgend ein Punkt $B$ von $g$ beschreibt, und verbinden die Punkte $B_{1}, B_{2} \ldots$ von $b$ mit den entsprectienden Lagen $A_{1}, A_{2} \ldots$ des Punktes $A$. $Z u$ dem Zwecke teilen wir den Kreis $b^{\prime}$ von $B_{0}^{\prime}$ aus in den Punkten $B_{1}^{\prime}, B_{2}^{\prime} \ldots$ in $2 n$ gleiche Teile und machen auf $a^{\prime \prime}$ die Strecken $A_{0}^{\prime \prime} A_{1}^{\prime \prime}, A_{1}^{\prime \prime} A_{2}^{\prime \prime} \ldots=\frac{\mathfrak{h}}{2 n}$. Pamit keine der so erhaltenen Erzengenden im Aufriß mit $a^{\prime \prime}$ znsammenfält, setzen wir $n$ gleich einer ungeraden Zahl, z. B. = 9. Cin ferner bei der Konstruktion ron $l^{\prime \prime}$ 
die auf $a^{\prime \prime}$ schon vorhandene Teilung $A_{0}^{\prime \prime}, A_{1}^{\prime \prime}, A_{2}^{\prime \prime} \ldots$ wieder benutzen zn können, wählen wir den Höhemunterschied der Punkte $A_{0}^{\prime \prime}$ und $B_{0}^{\prime \prime}$ gleich einem Vielfachen von $\mathfrak{1} \mathfrak{i}$, z. B. $=\frac{7}{18} \mathfrak{h}$.

Der zweite scheinbare Umriß der Fläche besteht aus unendlich vielen kongruenten hyperbelartigen Ästen mit den Asymptoten $g_{0}^{\prime \prime}, g_{9}^{\prime \prime}, g_{1}^{\prime \prime} \ldots$ Diese $\ddot{A}$ ste berïhren die Gerade $a^{\prime \prime}$ in den Mittelpunkten der Strecken $A_{0}^{\prime \prime} A_{9}^{\prime \prime}, A_{9}^{\prime \prime} A_{18}^{\prime \prime} \ldots$; sie berühren anßerdem die Sinuskurve $b^{\prime \prime}$ und sind in einiger Entfernung von $a^{\prime \prime}$ so schwach gekrümmt, daß sie nahezu als geradlinig betrachtet werden können.

Die Schnittpunkte der zu $\Pi_{2}$ parallelen Geraden $g_{0}, g_{9}, g_{18} \ldots$ beschreiben die Doppelkurven der Fläche; die erste geht durch die Schnittpunkte von $g_{9}$ mit $g_{0}$ und $g_{18}$.

167. Eine Schraube ist ein Körper, der durch Schraubung einer allseitig begrenzten Fläche erzeugt wird. Gewöhnlich besteht die Schraube ans einem geraden Kreiszylinder als Kern und dem auberhalb anliegenden Gewinde. Dieses bestimmt man am einfachsten

Fig. 156 .
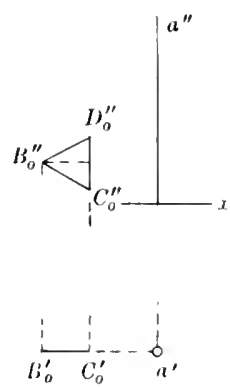

Fig. 157 .
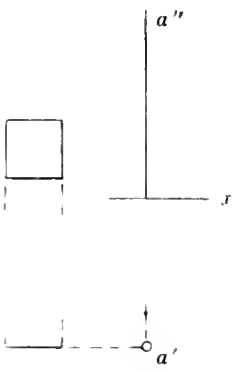

durch Angabe eines Meridianschnitts, und dazu läßt sich jede Figur verwenden, die eine gerade Seite enthält, mit der sie auf dem Zylindermantel aufliegt. Die Ganghöhe der Schraubung ist dann so groß zu wählen, da@ die in dieselbe Ebene fallenden Lagen der Figur sich nicht gegenseitig durchschneiden. - Unter Schraubenmutter versteht man einen Körper mit einem Hohlraum, den die zugehörige Schraube ausfüllt, so daf sie in ihm eine Schraubnng ansführen kann, bei der die Oberflächen der beiden Körper aufeinander gleiten.

Die Schraube mit scharfem Gewinde hat als Meridianschnitt ein gleichschenkliges Dreieck $B C D$, dessen Grundlinie $C D$ zur Achse $a$ parallel ist. Die Ganghöhe ist gleich der Grundlinie. Jeder Schenkel des Dreiecks beschreibt einen Teil einer schiefen geschlossenen Regelschraubenfläche. - In Fig. 156 ist $a \perp \Pi_{1}$. Um die Schraube in Grundund Aufrib zu zeichnen, konstruieren wir zunächst die Schraubenlinien $b$ und $c$, die von $B$, sowie von $C$ und $D$ erzeugt werden. Der zweite scheinbare Umrif der beiden Schraubenflächen ist zwar krummlinig, aber nach Art. 166 ron einer Geraden so wenig verschieden, daßs wir ihn durch die gemeinsamen Tangenten der Sinnskurven $b^{\prime \prime}$ und $c^{\prime \prime}$ 
ersetzen dïrfen. Der Lmrib ist also nach aubeu abgerundet, d. h. ohne Ecken; in der Nähe des Kernzylinders ïberschneidet die eine Umriblinie die andere, jedocl so, dab die Schnittpunkte noch auberhalb des Zylinderumrisses liegen.

Bei der Darstellung der anfgeschnittenen Schraubenmutter, die durch die Meridianebene $\| \Pi_{2}$ begrenzt wird, tritt an die Stelle der eben ermittelten Umriblinie der aus aneinandergereihten Dreiecken gebildete Meridianschnitt.

Die Schraube mit flachem Gewinde wird durch ein Rechteck erzeugt, dessen Ebene die Achse a enthält, und von dem zwei Seiten If $a$ sind (Fig. 15\%). Die Ganghöhe ist in der Regrel doppelt so grob, wie die zur Achse parallele Rechtecksseite. Die auf der Achse senkrechten Seiten beschreiben Teile ron Wendelflächen. - Bei einer Wendeltreppe ist die Grundform der Wange, in der die Stufen befestigt sind, der Gewindeteil einer flachgängigen Schraube mit grober Ganghöhe. Ein von zwei Meridianschnitten begrenztes Stück des Gewindes wird dann als Treppenkr ümmling bezeichnet.

168. Die Wendelfläche als Fugenfläche bei einem schiefen Brückengewölbe (Fig. 158). Ein halber gerader Kreiszylinder sei begrenzt durch die in $\Pi_{1}$ liegeuden Mantellinien $A_{1} A_{2}$ nnd $B_{1} B_{2}$ und durch zwei kongruente Halbellipsen $k_{1}$ und $k_{2}$, deren Ebenen schräg

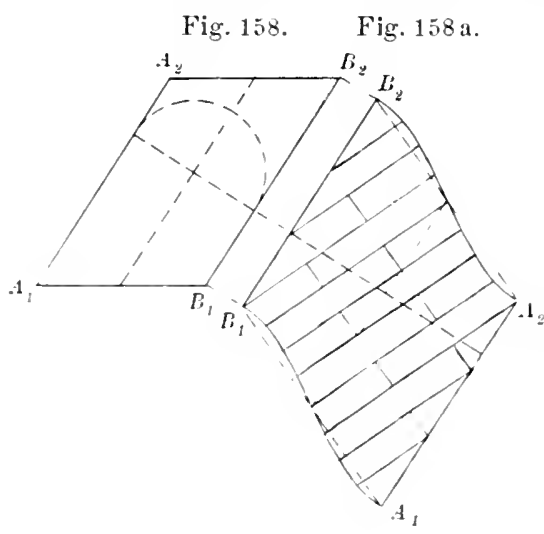
gegen die Mantellinien aber $\perp \Pi_{1}$ gestellt sind. Wollen wir diesen Halbzylinder als Laibungsfläche eines aus Quadern gebildeten Tounengewölbes benutzen, so dürfen wir die Fngen njcht in die Mantellinien legen. Bei einer solchen Anordnung der Quader würde nämlich der Druck überall senkrecht gegen die ebenen Fugenflächen wirken, die durch die Mantellinien und die Zylinderachse gingen; dächten wir uns also den Zylinder senkrecht zu seiner Achse mit zwei Ebenen durch die Punkte $A_{2}$ und $B_{1}$ geschnitten, so hielte sich zwar der zwischen ihnen liegende Teil des Gewïlbes in Gleichgewicht, nicht aber die au Serhalb übrig bleibenden Stücke. Wir miissen daher an Stelle der Mantellinien krumme Linienals Fugen verwenden, die so zu wählen sind, daß sie auf den Stirnkurven $k_{1}$ und $k_{2}$ annähernd senkrecht stehen. Dazu bedienen wir uns am einfachsten der auf dem Zylinder liegeuden Schraubenlinien, weil diese in der Abwickelung als Geraden erscheinen und deshall, ohne weiteres konstruiert werden können.

Der abgewickelte Zylinclermantel ist in Fir. 158 a in bekannter Weise gezeichnet worden (rgl. Art.94). Dabei hat sich die Halbellipse $l_{1}$ in eine halbe Sinuskurve mit den Scheiteln $A_{1}$ und $B_{1}$ verwandelt; 
der Wendepunkt liegt in der Mitte der Strecke $A_{1} B_{1}$, und seine Tangente bildet mit der Mantellinie einen Winkel $=\angle A_{2} A_{1} B_{1}$. Da die verwandelte Kurve von der Geraden $A_{1} B_{1}$ nur unerheblich abweicht, so ziehen wir die abgewickelten Lagerfugen geradlinig und $\perp A_{1} B_{1}$, mithin die Stobfugen $\| A_{1} B_{1}$. Die so erhaltenen Geraden werden mittels der abgewickelten Mantellinien auf den Zylinder übertragen und liefern dort zwei Scharen von Schraubenlinien, die einander rechtwinklig schneiden. Die Fugenflächen der einzelnen Wölbsteine werden durch die Zylindernormalen in den Punkten dieser Schranbenlinien gebildet, sind also Wendelflächen.

169. Die Röhrenschraubenfläche (Serpentiue) ist die Einhüllende einer geschraubten Kugel (Fig. 159). Stellen wir die Schraubenachse $a$ wieder $\perp \Pi_{1}$ und bezeichnen mit $b$ die Schraubenlinie, die der Kugelmittelpunkt $B$ beschreibt, so erhalten wir als zweiten scheinbaren Umriß der Fläche die Einhüllende $v^{\prime \prime}$ aller Lagen rer bewegten Kugel, also eine Parallelkurve der Sinuskurve $l^{\prime \prime}$ (vgl. Art. 145). Beide Kurven haben in entsprechenden Punkten denselben Krümmungsmittelpunkt; wir benutzen insbesondere die Krümmungsmittelpunkte in den Scheiteln von $b^{\prime \prime}$ zum Zeichnen von $v^{\prime \prime}$. Den Wendepunkten von $b^{\prime \prime}$ entsprechen Wendepunkte von $v^{\prime \prime}$ mit parallelen Tangenten. Ähnlich wie der Umriß einer schief gestellten Ringfläche kaun die Kurve $v^{\prime \prime}$ Überschneidungen aufweisen und Rückkehrpunkte besitzen, die auf der Evolute von $b^{\prime \prime}$ liegen.

Jede Lage der geschraubten Kugel berührt die Röhrenfläche in einem Hauptkreise $k$, dessen Ebene auf der Tangente ron $b$ senkrecht steht. In der Anfangslage $B_{0}$ des Punktes $B$ ist diese Tangente $\| \Pi_{2}$, also $k_{0}^{\prime \prime}$ eine Gerade. Die Fläche kann auch durch Schraubung des Kreises $k$ erFig. 159 .

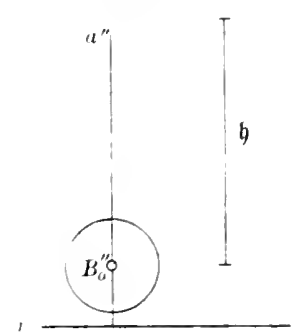
zeugt werden. Da seine Ebene mit der $\Pi_{1}$ beständig denselben Winkel bildet, so erscheinen alle Lagen ron $k$ im Grundrib als kongruente Ellipsen.

Meridian- und Normalkurve, wie überhaupt alle ebenen Schnitte der Fläche werden am einfachsten als die Eiuhüllenden der Kreise konstruiert, in denen die betreffende Ebene eine Reihe von Lagen der erzeugenden Kugel schneidet.

\section{Eigenschattengrenzen.}

170. Eine rechtsgängige Schraubenfläche sei gegeben durcn ihre Achse $a \perp \Pi_{1}$, die Ganghöhe $\mathfrak{h}$ und eine beliebige Lage der Erzeugenden $c$. Fig. 160 zeigt nur den Grundrib; dabei bedeutet $A_{0}$ den Schnittpunkt von $a$ mit $\Pi_{1}$. Machen wir auf $u$ die Strecke $A_{0} S$ gleich der reduzierten Ganghöhe $\frac{\mathfrak{h}}{2 \pi}$ und bezeichnen mit $S_{h}$ den ersten Spurpunkt des 
durch $S$ gehenden Lichtstrahls 7 , so ist durch Angabe von $S_{h}$ die Lichtrichtung bestimmt.

Der Kreis $k^{\prime}$ um $A_{0}$ sei der Grundriß irgend einer Schraubenlinie $k$ der Fläche; wir stellen uns die Aufgabe, die auf $k$ befindlichen Punkte ler Eigenschattengrenze $s$ zu ermitteln. Der Schnittpunkt $P$ von $c$ und $k$ würde der Kurve $s$ angehören, wenn seine Berührungsebene $T$, die durch die Tangenten $e^{2}$ und $t$ von $c$ und $k$ bestimmt ist, zu $l$ parallel wäre, oder mit anderen Worten, wenn die durch $S \| T$ gelegte Ebene $T_{1}$ den Strahl $l$ enthielte. $U m T_{I} z u$ bestimmen, ziehen wir durch is Parallelen zu $e$ und $t$. Die erste schneidet $\Pi_{1}$ in einem Punkte der

Fig. 160 .

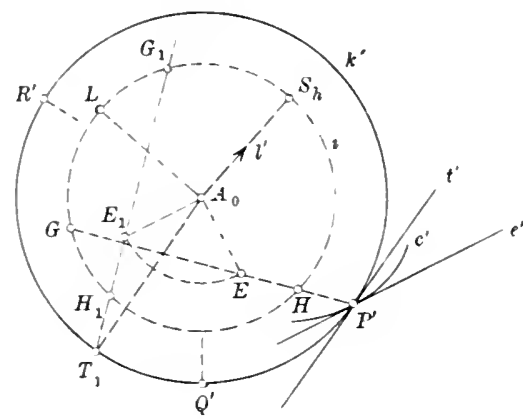
Parallelen durch $A_{0}$ zи $e^{\prime}$, z. B. in $E_{1}$; die Grundribspur $T_{1}$ der zweiten liegt auf dem Kreise $k^{\prime}$ $\left(A_{0} T_{\mathrm{I}} \| t^{\prime}\right)$. Dann ist $E_{\mathrm{I}} T_{\mathrm{I}}$ die Grundribspur von $T_{1}$, und diese Gerade mübte durch $S_{h}$ gehen, wenn sich der Punkt I' auf $s$ befinden würde. Gegenwärtig ist dies nicht der Fall; verstehen wir jedoch unter $G_{1}$ und $H_{1}$ die Schnittpunkte ron $E_{1} T_{1}$ mit den Kreise $i$, der $A_{0}$ zum Mittelpunkte und $A_{0} S_{h}$ zum Radius hat, so bedarf es nur einer rechtsgängigen Schraubung der Kurve $c$ um den überstumpfen Winkel $G_{1} A_{0} S_{h}$ oder um den Winkel $H_{\mathrm{I}} A_{0} S_{h}$, um den Punkt $I^{\prime}$ auf die Kurve s zu bringen. Machen wir daher $\angle P^{\prime} A_{0} Q^{\prime}=-G_{1} A_{0} S_{h}$ und $-l^{\prime \prime} A_{0} R^{\prime}$ $=\angle I_{1} A_{0} S_{h}$, so erhalten wir auf $k^{\prime}$ zwei Punkte $Q^{\prime}$ und $R^{\prime}$ von $s^{\prime}$.

Um die gefundene Konstruktion zu vereinfachen, wollen wir zuvor jeder beliebigen Richtung des Raumes einen ganz bestimmten Punkt der Ebene $\Pi_{1}$ in folgender Weise zuordnen: Wir definieren als der Richtung $m$ zugeorduet denjeuigen Punkt $M$ ron $\Pi_{1}$, den wir erbalten, wenn wir die Gerade $S M_{1} \| m$ bis $\Pi_{1}$ ziehen und clen Punkt $M_{1}$ um $A_{0}$ im Sinne rler aufwärts gehenden Schraubung (im vorliegenden Falle also entgegengesetzt dem Sinne des ('hrzeigers) um $90^{\circ}$ drehen. Konstruieren wir hiernach in Fig. 160 die den Richtungen $e, t, l$ in $\Pi_{3}$ zugeordneten Punkte $E, T, L$, so fällt $T$ mit $F^{\prime}$ zusammen, und $I$ liegt auf dem Kreise $i$. Dann ergibt sich auf Grund der vorhergehenden Darlegungen die Regel: Um a uf einer Schraubenlinie $k$ der Fläche die Punkte der Eigenschattengrenze $s$ zu finden, zielue man in einem beliebigen Punkte $T^{\prime}$ yon $k$ die 'Tangente e der Erzeugenden r und hestimme in $\Pi_{1}$ die den Richtungene und $?$ zugeordneten Punkte $E$ und $L$. Ferner zeichne man in $\Pi_{1}$ den mit $l^{\prime}$ konzentrischen Kreis $i$ durch $L$. Schneidet die Gerade $P^{\prime} E$ den Kreis $i$ in $G$ und $I I$, so geht $P$ durch Schraubung um den Winkel $G A_{0} L$ oder $M A_{0} L$ in die Punkte $Q$ und li von $s$ über.

Geht die Gerade EI' durch $I$. so ist $I^{\prime}$ selbst ein Punkt der hurres. 
171. Ist $c$ die Normalkurve der betrachteten Schraubenfläche, so tritt an die Stelle von $E$ der unendlich ferne Punkt des Lotes von $A_{0}$ auf $e^{\prime}$, und die Gerade $E P^{\prime}$ wird zur Normale von $e^{\prime}$ in $P^{\prime}$. Dann folgt aus dem letzten Satze in Art. 170: Der Grundriß $s^{\prime}$ der Eigenschattengrenze ist der Ort derjenigen Punkte der Grundrisse aller Normalkurven, deren Normalen durch den Punkt $I$, of ehen.

Bei der geraden geschlossenen Regelschraubenfläche ist demnach die Kurve $s^{\prime}$ der Ort rer Fubpunkte aller Lote von $I$ auf die durch $A_{0}$ gehenden Geraden, d.h. ein Kreis vom Durchmesser $A_{0} L$. I)a jener Fußpunkt den Kreis $s^{\prime}$ einmal vollständig durchlänft, wenn der Grundriß der Erzengenden eine halbe Drehung um $A_{0}$ ausführt, so erhalten wir als Eigens chattengrenze $s$ eine Schraubenlinie von dex Ganghöhe $\frac{\mathfrak{l}}{2}$.

172. Bei einer schiefen Regelschraubenfläch $\Theta$ fällt für jeden Punkt einer Erzeugenden die Tangente $e$ mit dieser Erzeugenden zusammen, und die Punkte $E_{1}$ und $E$ liegen für alle Erzeugenden auf einem Kreise $f \mathrm{um} A_{0}$. Um also für eine beliebige Erzeugende $e$ den Punkt $P$ zu konstruieren, den sie mit $s$ gemein hat, ziehen wir im vorher bestimmten Sinne die Gerade $A_{0} E \perp e^{\prime}$ bis $f^{\circ}$ und schnejden $e^{\prime}$ mit $L E$ in $P^{\prime}$.

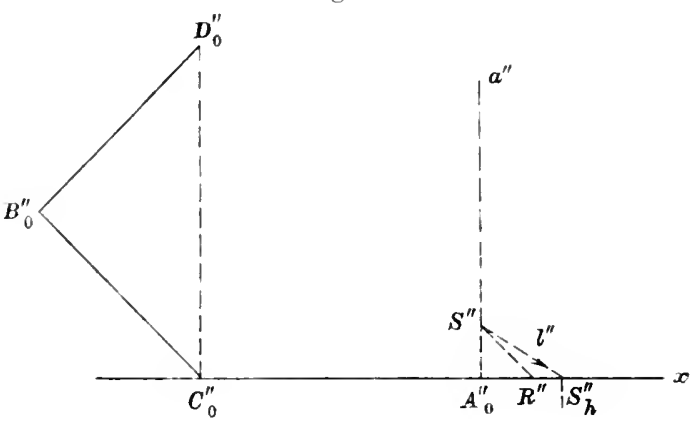

Fig. 161.

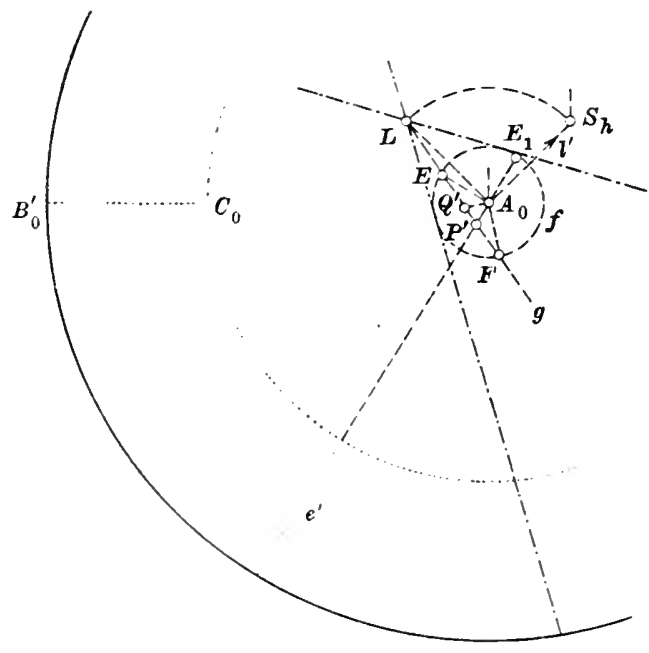

Anwendung auf die scharfgängige Schraube. In Fig. 161 ist die Schraube - wie vorher in Art.167 - durch den zu $\Pi_{2}$ parallelen Meridianschnitt $B_{0} C_{0} D_{0}$ gegeben, die Ganghöhe h ist wieder $=C_{0} D_{0}$. $\mathrm{Cm}$ für die von der Strecke $B_{0} C_{0}$ beschriebene Fläche die Eigenschattengrenze $s$ zu konstruieren, machen wir auf $a^{\prime \prime}$ die Strecke $A_{0}^{\prime \prime} S^{\prime \prime}=\frac{\mathfrak{h}}{2 \pi}$ und bestimmen wio früher die Punkte $S_{h}$ und $L$. Wir 
ziehen ferner $S^{\prime \prime} R^{\prime \prime} \| B_{0}^{\prime \prime} C_{0}^{\prime \prime}$ bis $x$ und beschreiben in $\Pi_{1}$ mit $A_{0}^{\prime \prime} R^{\prime \prime}$ den Freis $f^{\prime}$ um $A_{0}$. Ist nun $e^{\prime}$ der Grundriß einer beliebigen Erzeugenden $e, E_{1}$ der Schnittpunkt von $f$ mit der Verlängerung von $e^{\prime}$ über $A_{0}$, so erhalten wir anf $f$ den der Richtung $e$ zugeordneten Punkt $E$ durch Vierteldrehung vou $A_{0} E_{1}$ entgegengesetzt dem Sinne des Uhrzeigers, und dann bestimmt die Gerade $I, E$ auf $e^{\prime} \operatorname{den}$ Punkt $I^{\prime}$ ron $s^{\prime}$. Ziehen wir ungekehrt zuerst durch $L$ eine beliebige Gerade $y$, die $f$ in $E$ und $F$ schneidet, so finden wir auf ils zwei Punkte von s', nämlich ihre Schnittpunkte $P^{\prime}$ und $Q^{\prime}$ mit den Loten in $A_{0}$ zu $A_{0} E$ und $A_{0} F$. Berührt $g$ den Kreis $i$, so liegen $P^{\prime}$ und $Q^{\prime}$ beide unendlich fern, d. h. die 'Tangenten aus $L$ an $f$ sind Asymptoten von $s^{\prime}$. Gegenwärtig kommt nur der Teil von $s$ ' in Betracht, den die um $A_{0}$ durch $B_{0}^{\prime}$ und $C_{0}$ beschriebenen Kreise einschließsen, und dieser kann durch die Asymptoten angenähert ersetzt werden. - Die Kurve $s^{\prime}$ hat in $A_{0}$ einen Selbstberührungsponkt und in $J$ einen loppelpunkt, wie sich sofort ergibt, wenn die Gerade $g$ dureh $A_{0}$, bzw. durch die Endpunkte des auf $\mathcal{A}_{0} L$ senkrechten Durchmessers von $f$ gezogen wird.

173. Der Schlagschatten, den eine Schraubentläche von ihrer Eigenschattengrenze und ron ihrem Rande empfängt, wird nach dem indirekten Verfahren, also aus dem schlagschatten auf die $\Pi_{1}$ ermittelt. Noch einfacher, obgleich weniger genau, läbt sich die ganze Schattenkonstruktion auch in der Weise behandelı, dafo man die Fläche in der Lichtrichtung mit vertikalen Hilfsebenen schneidet (Art. 124, I).

\section{Windschiefe Flächen.}

174. Nach Art. 89 verstehen wir unter einer Regelfläehe eine solche Fläche, die durch Bewegung einer Geraden erzeugt wird. Um das Gesetz dieser Bewegung in jedem einzelneu Falle festzulegen, schreiben wir am einfachsten drei bestimmte Leitkurven $l_{1}, l_{2}, l_{3}$ vor, welche die Erzeugende beständig schneiden soll. Dann erhalten wir nämlich die durch irgend einen P'unkt $A_{1}$ von $l_{1}$ gehenden Erzeugenden als die gemeinschaftlichen Mantellinien der beiden Kegelflächen, die $A_{1}$ zur Spitze und $l_{2}$ bzw. $l_{3}$ zu Leitkurven haben. Die so entstehende Pegelflache ist in allgemeinen windschief; denn würden zwei unendlich benachbarte Erzeugende, die mit $l_{1}, l_{2}, l_{3}$ bzw. die I'unkte $\boldsymbol{A}_{1}$, $A_{2}, A_{3}$ und $B_{1}, B_{2}, B_{3}$ gemein haben, einander schneiden, so müßten die Gerarlen $A_{1} B_{1}, A_{2} B_{2}, A_{3} B_{3}$, d. h. die Tangenten der drei Leitkurven in $A_{1}, A_{2}, A_{3}$, in einer und derselben Ebene liegen. Dies wird im allgemeinen nicht der Fall sein; ereignet es sich für gewisse Lagen der Erzeugenden, so besitzt die Fläche in einer solchen Erzeugenden ein ebenes Flächenelement, und findet es bei besonderer Auswalıl der rrei Leitkurven für alle Erzengenden statt, so ist die Fläehe abwiekelbar.

175. Verzeichnen wir auf irgend einer windschiefen Fläche eine Keihe von unendlich dicht aufeinanderfolgenden Erzengenden $e, f, g$, $h, i \ldots$ und legen durch eine von ihnen, etwa $g$, eine beliebige Ebene $T$, so scheidet diese die Fläche in einer Kurve $s$, die durch die unendlich benachbarten Schnittpunkte $E . F, H, I$ von T mit $\rho, f, h, i$ 
hindurchgeht. Die Kurve $s$ wird zwischen $F$ und $H$ von der Geraden $g$ in einem Punkte $G$ getroffen, und dann ist $G$ ein Knotenpunkt der vollständigen, aus $g$ und $s$ bestehenden Schnittkurve, die $T$ mit der Fläche gemein hat (vgl. Art. 137). Die Ebene T berührt demnach die Fläche in dem (hyperbolischen) Punkte ( $\dot{r}$, und das Analoge gilt offenbar von jeder anderen, durch eine Erzengende gelegten Ebene.

Bei Anfgaben über ebene Schnitte, Durchdringnngen usw. bedienen wir uns naturgemäß stets der Erzeugenden der Fläche. Ebenso können wir die Eigenschattengrenze mit Hilfe ebener Schuitte, die zu einer projizierenden Ebene des gegebenen Lichtstrahls parallel sind, wenigstens angenähert ermitteln (Art. 124, I).

176. Je nachdem sich unter den drei zur Bestimmung der Fläche elforderlichen Leitkurven gerade Linien befinden oder nicht, können wir vier Arten von windschiefen Flächen unterscheiden.

a) Sind alle drei Leitlinien gerade und sämtlich windschief zueinander, so bezeichnen wir die Fläche als ein einschaliges Hyperboloid. Wir konstruieren sie am einfachsten, indem wir durch die Leitgerade $l_{1}$ ein Ebenenbüschel legen und die Schnittpunkte jeder einzelnen Ebene mit $l_{2}$ und $l_{3}$ bestimmen; ihre Verbindungslinie ist eine Erzeugende der Fläche. Durch jeden Punkt $A_{1}$ von $l_{1}$ geht eine Erzeugende, nämlich die Schnittlinie der Ebenen $A_{1} l_{2}$ und $A_{1} l_{3}$. - Zwei Erzeugende können einander niemals schneiden, denn wäre dies der Fall, so lägen auch die drei Leitgeraden in der durch die beiden Erzengenden bestimmten Ebene. Die Fläche ist also windschief.

Die vorliegende Fläche, deren weitere Behandlung wir in die projektive Geometrie verweisen, ist offenbar eine Verallgemeinerung des früher betrachteten einschaligen Umdrehungshyperboloids. Einen zweiten, technisch wichtigen Sonderfall bildet das hyperbolische Paraboloid, las sich ergibt, wemn die eine der drei Leitgeraden unendlich fern ist, also durch eine Richtungsebene ersetzt wird, zu der die sämtlichen Erzeugenden parallel sind. Dann verhalten sich die Abschnitte auf der einen Leitgeraden wie die zwischen denselben Erzengenden liegenden Abschnitte auf der andern.

Durch das in Fig. 162 dargestellte windschiefe Tiereck $A B C D$ wird ein hyperbolisches Paraboloid bestimmt, das $A B$ und $C D$ zu Leitgeraden und $B C$ und $A D$ zu Erzeugenden hat. Zeichnen wir nämlich das Parallelogramm $A D C E$, so können wir die Ebene $B C E$ als Richtungsebene des Paraboloids betrachten,

Fig. 162.

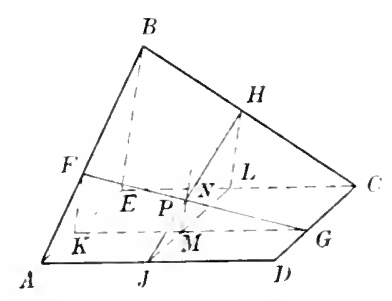
weil sie $B C$ enthält und zu $A D$ parallel ist. Wir erhalten also eine rritte Erzengende, indem wir $A B$ und $C D$ mit einer Ebene schneiden, die zu $B C E$ parallel ist. Legen wir diese Ebene durch den Punkt $K$ von $A E$, so schneirlet sie die Ebone $A B E$ in $K F \| E B$ und die Ebene $A D C E$ in $K G \| E C$, und dann ist $F G$ die gesuchte Erzengende. - Wir können aber durch dasselbe Viereck noch ein zweites hyperbolisches Paraboloid legen, indem wir $B C$ und $A D$ als Leit- 
geraden, $A T$ und $C D$ als Erzeugende und clemnach die Ebene $A B E$ als Richtungsebene auffassen; um von ihm eine dritte Erzeugende $I J J$ zu konstruieren, zjehen wir durch irgend einen l'unkt $I$ von $C E$ die Geraden $L H \| E B$ und $L . J \| E A$ bzw. bis $B C$ und $A I$. Die Ebenen $F G K$ und $I J J I$ schneiden sich in einer durch den Punkt $M=G K \times J I$. gehenden Geraden, die zu $E B$ parallel ist; sie möge $F(r$ in $N, I J J$ iu $l$ treffen. Damn rerhïlt sich

und

$$
\frac{M N}{K F}=\begin{aligned}
& \text { G } M \\
& G K
\end{aligned}
$$

$$
\frac{K F}{E B}=\frac{A K}{A E},
$$

also ist

$$
M N=\frac{E B \cdot G M \cdot A K}{G K \cdot A E}
$$

Ebenso ergibt sich

$$
M P=\begin{gathered}
E B . J M \cdot C L \\
J L \cdot C E
\end{gathered}
$$

Nun ist aber $J M=A K, C I=G M, J L=A E$ und $C E=G K$, also folgt $M N=M P$, d. h. die Punkte $N$ und $I^{\prime}$ fallen zusammen. Die Erzeugende $H J$ des zweiten Paraboloids schneidet also $F G$ und folglich alle Erzeugenden des ersten; sie liegt demmach ganz auf diesem. Dasselbe gilt offenbar ron allen Erzeugenden der zweiten Fläche, die beiden Paraboloide decken sich daher vollständig. Auf dem hyperbolischen Paraboloid gibt es also zwei Scharen gerader Linien.

Wie in Art. 140 wird bewiesen, daß das hyperbolische Paraboloid eine Fläche zweiter Ordnung ist.

Durch jeden Flächenpunkt gehen zwei Erzeugende, nämlich je eine von jeder Schar. Die durch sie bestimmte Ebene beriuhrt die Fläche in dem betrachteten Punkte.

Unter den Berïhrungsebenen des hyperbolischen Paraboloids leefindet sich auch die unendlich ferne Ebene des Raumes ${ }^{1}$ ): denn diese hat mit der in unserer Figur dargestellten Fläche die unendlich fernen Geraden der Ebenen $A B E$ und $B C E$ gemein und berïlurt folglich die Fläche in unendlich fernen P'unkte von $B E$. - Alle nicht berülirenden Ebenen schneiden die Fläche in Parabeln oder Hyperbehn, je nachdem sie zu $I E$ E parallel sind oder nicht.

177. Das hyperboliscbe Paraboloid wird in der Technik mannigfach angewendet, z. B. bei windschiefen Dächern. In Fig. 163 soll über dem in $\Pi_{1}$ ganz beliebig gezeichneten Viereck A I C I) ein Walındach konstruiert werden. Würden wir - wie in Art. 48 - durch alle vier Seiten gleich geneigte Ebenen legen, so liefe die Schnittlinie der durch $A B$ und ( I) gehenden Ebonen nach dem Schnittpunkte dieser Gerarlen, wir erhielten also ein Tach mit schiefem First. Lm

I Vel. die Anmerkung allf S. 11. 
das zu vermeiden, legen wir nuy durch $D A, A B$ und $B C$ je eine Ebene unter beliebiger, aber gleicher Tafelneigung; die so entstehenden Gratlinien $A E$ und $B F$ fallen im Grundriß in die Halbierungslinien der. Winkel bei $A$ und $B$. Zwischen beiden zielsen wir \| $A B$, im übrigen aber beliebig, die Gerade $E^{\prime} F^{\prime}$ als Grundrib des horizontalen Firstes $E F$ und legen als vierte Dachfläche durch $C D$ und $E F$ ein hyperbolisches Paraboloid, das also die $\Pi_{1}$ zur Richtungsebene hat. Die zwoite Richtungsebene kaun noch beliebig gewählt werdeu; nehmen wir sie $\perp E^{\prime} F^{\prime}$, so läuft die zugehörige Schar von Erzeugenden - die Dachsparren - senkrecht zum First, während die andere Schar, die den Dachlatten entspricht, horizontal ist. Iie Ebene $D A E$ schneidet das Paraboloid in einer krummen Gratlinie $D \boldsymbol{E}$, nämlich in einem

Fig. 163.

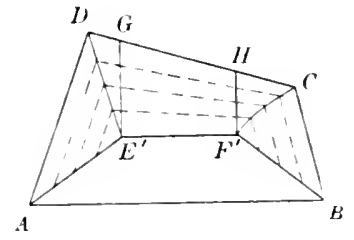

Fig. 164.

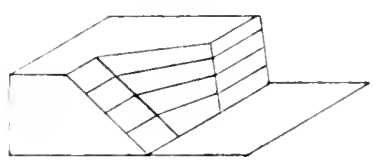

Parabelbogen, wenn die Gerade $A D$ zur Schnittlinie der Richtungsebenen parallel, d. l. $\perp A B$ ist, anderenfalls in einem Hyperbelbogen. Um diese Schnittkurve zu konstruieren, schneiden wir die beiden Flächen mit einer Schar horizontaler Hilfsebenen und ermitteln in jeder von ihmen den Schnittpunkt der Geraden, die sie mit beiden Flächen gemein hat. $\mathrm{Zu}$ dem Zwecke teilen wir die Erzeugenden E G und $F H$ des Paraboloids und die Kante $A E$ in gleich viele gleicher. Teile, verbinden die entsprechenden Teilpunkte von $E G$ und $F H$ und ziehen durch die Punkte anf $A E$ Parallelen zu $A D$.

Das hyperbolische Paraboloid wird ferner als Bösclungsfläche benutzt zur Vermittelung des Überganges von einer ebenen Böschung in eine zweite von anderem Neigungswinkel (Fig. 164).

178. b) Unter den windschiefen Flächen mit zwei Leitgeraden sind diejenigen bemerkenswert, bei denen die eine Leitgerade unendlich fern. also wieder durch eine Richtungsebene gegeben ist. Wir bezeichnen sie als Konoidflächen und verstehen insbesondere unter einem geraden Konoid ein solches, dessen endliche Leitgerade auf der Fig. 165. Richtungsebene senkrecht steht.

Darstellung eines geraden Kreiskonoids, das durch die vertikale Leitgerade $l$ und den in $\Pi_{2}$ liegenden Leitkreis $l i$ bestimmt ist (Fig. 165). Schneiden wir $l$ und $k$ mit einer horizontalen Ebene bzw. in $P$ und in $Q$ und $R$, so erhalten wir die Erzeugenden $P Q$ und $P I_{i}$ der Fläche. Den Endpunkten $A$ und $B$ des rertikalen, sowie $C$ und $D$ 
des horizontalen Kreisdurchmessers entsprechen vier ebene Flächenelemente $A G, B H, C E, D E$ mit horizontalen bzw. durch 7 gehenden

Fig. 166.

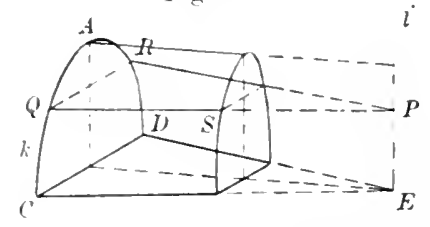
Berührungsebenen. Die Gerade $l$ bildet

$i$ von $G$ bis $I I$ eine Doppellinie der Fläche.

Jede zu $\Pi_{2}$ parallele Ebene schneidet das Konoid in einer Ellipse. Trifft nämlich die schneidende Ebene $\mathrm{E}$ die Erzeugende $P Q$ in $S$, so ist

$$
\begin{aligned}
& P^{\prime \prime} S^{\prime \prime} \\
& P^{\prime \prime} Q
\end{aligned}=\begin{aligned}
& \gamma^{\prime} S^{\prime} \\
& \gamma^{\prime} Q^{\prime}
\end{aligned}=\begin{aligned}
& \text { Abstand } 7 \mathrm{E} \\
& \text { Abstand } 7 \Pi_{2}
\end{aligned},
$$

also konstant für alle Erzeugenden. Nach Art. 51 ist daher der Aufrif. der Schnittkurve perspektiv affin zum Kreise $l$ mit $l^{\prime \prime}$ als Affinitätsachse, mithin eine Ellipse.

Das gerade Kreiskonoid dient als Laibungsfläche eines Tonnengewölbes über konvergierende Widerlagsmauern (Fig. 166).

179. c) Windschiefe Flächen mit einer Leitgeraden.

1. Die Wölbfläche des schrägen Durchgangs hat zu Leitlinien zwei parallel gestellte, gleich grobe Kreise $k_{1}$ und $k_{2}$ und eine zu

Fig. 167.

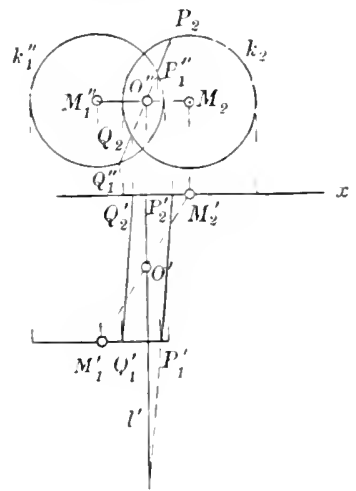

Fig. 168 .

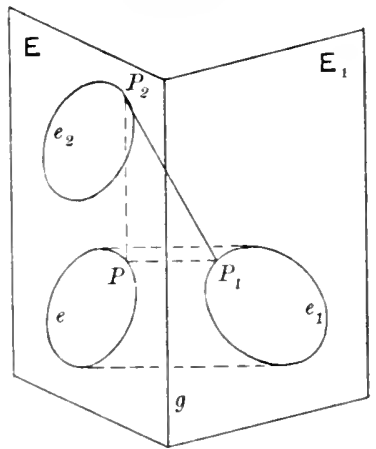

den Ebenen dieser Kreise senkrechte Gerade 7 durch den Mittelpunkt O' der Terbindungslinie der beiden Kreismittelpunkte $M_{1}$ und $M_{2}$. In Fig. 167 liegt $k_{2}$ in $\Pi_{2}$, und die Gerade $M_{1} M_{2}$ ist $\| \Pi_{1}$. Eine durch 7 beliebig gelegte Ebene schneidet $k_{1}$ in $P_{1}$ und $Q_{1}, k_{2}$ in $P_{2}$ und $Q_{2}$ : dann sind die zueinander parallelen Geraden $I_{1}^{\prime} I_{2}^{\prime}$ und $Q_{1} Q_{2}$ zwei Elzeugende der Fläche, während die Verbindungslinien $P_{1} Q_{2}$ und $Q_{1} I_{2}^{\prime}$ einem schiefen Kreiskegel mit $O$ als Mittelpunkt angehören. - Sollen die oberen Hälften der Kreise $k_{1}$ und $k_{2}$ mit einem Gewölbe überspannt werden, dessen Fugen geradlinig sind, so ist nach Art. 168 der durch lie Kreise gelerte Zylinder nicht verwendbar, wohl aber die soeben konstruierte windschiefe Fläche, weil bei iłır die Erzeugenden - wio der Aufric deutlich zeigt - auf den Stirnkurven naliezu senkrecht stehen. 
2. Das Zylindroid (Fig. 168). Wir schneiden einen Kreiszylinder mit den Ebenen $\mathbf{E}$ und $\boldsymbol{E}_{1}$ in den Ellipsen $e$ und $e_{1}$ und bezeichnen als entsprechend je zwei Punkte ron $e$ und $e_{1}$, die, wie z. B. $I^{\prime}$ und $I_{1}^{\prime}$, auf derselben Mantellinie liegen. Verschieben wir die Ellipse e parallel zur Schnittlinie $g$ von $\mathrm{E}$ und $\mathrm{E}_{1} \mathrm{~m}$ eine beliebige Strecke $J^{\prime} P_{2}$ in die neue Lage $e_{2}$, so bilden die Geraden, welche die Punkte von $e_{2}$ mit den entsprechenden Punkten von $e_{1}$ verbinden, ein Zylindroid. Dieses hat zur Leitgeraden die mendlich ferne Gerade der Ebene $P P_{1} P_{2}$. - Das Zylindroid findet Anwendung als Laibungsfläclıe des Gewölbes zur Unterstützung einer viereckigen Spindeltreppe (Fig. 169). An die Stelle von $e_{1}$ und $e_{2}$ treten hier kongruente HalbFig. 169. ellipsen, die in den Diagonalebenen des quadratischen Treppenhauses liegren. Jede Erzeugende der Fläche ist parallel zur vertikalen Seitenwand des Treppenhauses.

3. Die schiefe geschlossene Regelschraubenfläche.

d) Eine windschiefe Fläche ohne gerade Leitlinien ist z. B. die schiefe offene Regelschraubenfläche.

\section{Grundzüge der Beleuchtungslehre.}

180. Den früher ausgeführten Schattenkonstruktionen lag die Absicht zugrunde, die Anschaulichkeit der durch Projektion erhaltenen Bilder durch Wiedergabe der Beleuchtungsverhältnisse zu erhöhen. Wir erreichen diesen $Z$ weck anf vollkommenere Weise, wem wir nicht nur die Grenzlinien zwischen Licht und Schatten, sondern auch die Abstufung der Helligkeit auf den beleuchteten Oberflächenteilen darstellen. Dabei betrachten wir ausschließlich den Fall der Parallelbeleuchtung. Dann ist die Beleuchtungsstärke eines (ebenen) Flächenelements proportional dem Kosinus des Winkels, den die Flächennormale mit der Lichtrichtung bildet. Bezeichnen wir diesen Einfallswinkel mit $\lambda$ und setzen die Beleuchtungsstärke einer zur Lichtrichtung senkrechten Ebene $=1$, so ist diejenige des betrachteten Flächenelements $=\cos \lambda$. - Wir nehmen ferner an, die Oberfläche des beleuchteten Körpers sei vollkommen matt (nicht poliert), so daß sie an jeder Stelle das einfallende Licht nach allen Richtungen hin zerstreut und nicht in bestimmter Richtung reflektiert. In diesem Falle ist die Helligkeit, in der ein Oberflächenelement unserem Auge erscheint, unabhängig von der Sehrichtung, also gleich seiner Beleuchtungsstärke $\cos \lambda$.

181. Die Helligkeit einer Ebene ist überall dieselbe.

Auf einer krummen Fläche bilden alle Punkte von bestimmter Helligkeit eine gewisse Kurve, die als Lichtgleiche (Isophote) bezeichnet wird. - Die Lichtgleichen einer abwickelbalen Fläche sind ihre Erzengenden.

Die Darstellung der Helligkeitsverteilung im Bilde einer krummen Fläche erfolgt durch Auftragen verschiedener Farbentöne. Um hierfür eine geometrische Grundlage zu gewinnen, konstruieren wir zunächst 
eine Anzahl von lichtgleichen, etwa diejenigen sechs, die den Werten $\cos \lambda=1, \begin{array}{llll}4 & 3 & 2 & 1 \\ 5 & 5 & 5 & 5\end{array}, 0$ entsprechen. Wir bezeichnen sie mit den Zahlen $0,1 \ldots 5$, indem wir durch jede Zahl den Hunkelheitsgrad ausdrïcken, der zwischen der betreffenden Lichtgleiche und der nächstfolgenden durch Tuschlagen hervorzubringen jst. Auf einer nicht abwickelbaren Fläche giht es im allgemeinen nur einzelne Punkte von der Helligkeit 1 (Helligkeitspole); die Helligkeit 0 kommt dem im Eigenschatten befundichen Flächenteile zu. Wir kömmen auch für diesen Teil, entsprechend den Kosinuswerten $\frac{4}{5}, \frac{3}{5} \cdots$, eine Reihe von Kurven gleicher Neigung der Flächemnormale gegen den Lichtstrahl konstruieren. Um ihnen eine physische Bedeutung unterzulegen, macht man zuweilen die - allerdings ganz willkürliche - Annahme, das von der Luft und von den ungebenden Flächen in den Schattenraum hinein zerstreute Licht sei dem direkt einfallenden genan entgregengesetzt und von halb so grober Intensität wie jenes. Damn sind die eben erwälniten Kurven Orte gleicher Abnahme der Dunkelheit um je eine halbe Stufe.

182. Für die Darstellung der Helligkeit auf den meisten technischen Objekten bjldet die Beleuchtung der Kugel eine bequeme Grundlage (Fig. 170).

Die Lichtgleichen der Kugel sind Kreise, deren Ebenen a uf der Lichtrichtung senkrecht stehen. Ist 7 der durch den

Fig. 170 .
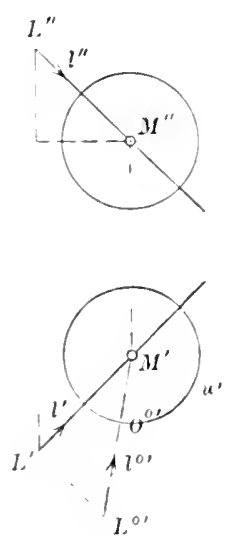
Kugelmittelpunkt $M$ gehende Lichtstrahl, $O$ der auf ihm liegende Helligkeitspol, so erhalten wir die Mittelpunkte $M_{1} \ldots M_{4}$ der mit $1 \ldots+$ bezeichneten Lichtgleichen durch Teilung der Strecke $O M$ in fünf gleiche Teile; denn für alle Punkte des Kingelkreises, dessen Ebene z. B. in $M_{3}$ auf $l$ senkrecht steht, bilden die zugehörigen Flächennormalen, d. h. die Kuggelradien, mit l einen Winkel, dessen Kosinus gleich ist $M_{3} M$, dividiert durch den Kugelradius, d. i. $=\frac{2}{5}$.

Un die Kreise 1,2... in Grund- und Aufrif zu zeichnen, drehen wir den Hauptkreis $u$, den die erste projizierende Ebene von l aus der Kugel schneidet, mit der Geraden 7 um seinen horizontalen D)rchmesser, bis er mit dem ersten wahren Unrils $u$ der Kugel \%usammenfällt ${ }^{1}$ ). Hierdurch grelangt ein beliebiger P'unkt $L$ von 7 nach $I^{0}$ und $l$ nach $l^{0}$; dabei ist $I^{\prime} L^{0^{\prime}} \perp l^{\prime}$ und $=L^{\prime \prime} L_{x}-M^{\prime \prime} M_{x}$. Die Cierade $70^{\prime}$ schneidet $u^{0^{\prime}}=u^{\prime}$ in $O^{0^{\prime}}$; durch Einteilung von $O^{0^{\prime}} M^{\prime}$ ergeben sich die Punkte $M_{1}^{0^{\prime}}, M_{2}^{0 \prime} \ldots$ Ziehen wir in $w^{0^{\prime}}$ durch $M_{3}^{\prime \prime \prime}$ die Sehne $C_{3}^{0 \prime} I_{3}^{0^{\prime}} \perp l^{0^{\prime}}$, so ist $C_{3}^{n} D_{3}^{n}$ die Umlegung des in $w$ liegenden l)urchmessers des Kreises $: ;$ und wir erhalten als Grundrils dieses lireises eine Ellipse 3 mit der

1) Wir könnten auch eine dritte Projektionsebene einführen, die zur Fune von " parallel ist, nder den Kreis " um seinen vortikalen Durchmesser drehen, bis ar $\| \Pi_{2}$ wird (vgl. Art. 87). 
groben Achse $A_{3}^{\prime} B_{3}^{\prime}=C_{3}^{n \prime} D_{3}^{\emptyset \prime}$ und der kleinen Achse $C_{3}^{\prime} I_{3}^{\prime}$. Mit dem Umris " hat der Kreis 3 wei Punkte $T_{3}, U_{3}$ semein; wir finden sie auf der Schnittlinie rer Ebenen beider Kreise, die im Schnittpnnkt $E_{3}$ von $C_{3} D_{3}$ mit dem lorizontalen llurchmesser von $w$ anf diesem senkrecht steht $\left(E_{3}^{\prime}=l^{\prime} \times C_{3}^{\prime \prime \prime} J_{3}^{0 \prime}, T_{3}^{\prime} U_{3}^{\prime} \perp l^{\prime}\right)$.

Die Ellipsen 1', $2^{\prime} \ldots$ sind oinander äbulich in paralleler Láge. ller Aufrib : $3^{\prime \prime}$ wird aus $3^{\prime}$ in bekannter Weise konstruiert (Art. 87): für Jicht iu der Richtung rler Würfeldiagonale sind $3^{\prime}$ und $3^{\prime \prime}$ kongruent.

183. Mit Hilfe der bereits gezeichneten Lichtgleichen einer Kugel konstruieren wir für dieselbe Lichtrichtung die Lichtgleichen einel beliebigen Umalrehungsfläche mit vertikaler Achse " und dem Lmribmeridian $m$ (Fig. 171). Um für den Parallelkreis $p$, der im Aufrib als die Strecke $P^{\prime \prime} Q^{\prime \prime}$ erscheint, die Lichtgleichenpunte zu bestimmen, ziehen wir an $m^{\prime \prime}$ die Tangente $P^{\prime \prime} S^{\prime \prime}$ bis $a^{\prime \prime}$ und parallel zu ihr an den zweiten scheinbaren Umriß der Kugel

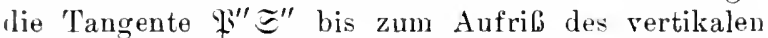
Kugeldurchmessers, ferner durch den Berührungspunkt

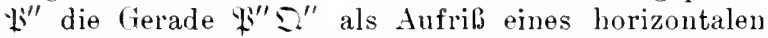

Fig. 171.

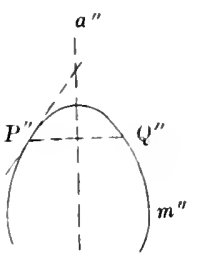

Kugelkreises p. Dann haben Kugel und UmdrehungsHäche in je zwei Punkten von $p$ und $p$, deren Verbindungslinien bzw. mit 5 und $S$ einander parallel sind, parallele Berührungsebenen und folglich gleiche Helligkeit. Wir brauchen demnach nur zu den Schnittpunkten von $p$ mit den Kugellichtgleichen die entsprechenden Punkte auf $p$ zu ermitteln.

184. Auch die Helligkeit einer ebenen Fläche ist leicht zu bestimmen, sobald für irgend eine Hilfskugel die Lichtgleichen konstruiert sind. Sei $\mathrm{E}$ eine beliebige Ebene mit der Grundrißspur $e_{1}$ und dem ersten Neigungswinkel $\varepsilon_{1}$, $f$ eine Fallinie von $\mathrm{E}$, fo ihre Umlegung um $f^{\prime \prime}$ in $\Pi_{1}$, also $f^{\prime} \perp e_{1}$ und $-f^{\prime \prime} f_{0}=\varepsilon_{1}$ (Fig. 17\%). Um auf der Hilfskugel denjenigen Punkt zn ermitteln, der dieselbe Helligkeit besitzt wie $E$. fällen wir vom Kugelschnittpunkte $M$ auf $E$ ein Lot $n\left(n^{\prime} \perp e_{1}\right)$ und bestimmen seinen Schnittpunkt $P$ mit der belenchteten Halblugel. Die Fig. 172.

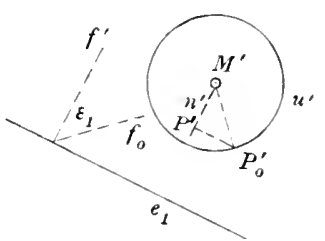
erste projizierende Ebene von $n$ selineidet die Kugel in einem Hauptkreise $k$, den wir um seinen horizontalen Durchmesser in den Hauptkreis $u$ umlegen. Ziehen wir dam $M^{\prime} P_{0}^{\prime} \perp f_{0}$ bis $l_{0}^{\prime}=u^{\prime}$, so ist $P^{\prime}$ der Fubpunkt des Lotes von $P_{0}^{\prime}$ auf $n^{\prime}$.

\section{Kotierte Projektion und topographische Flächen.}

185. Wir können die Lagre eines Punktes $P^{\prime}$ in Raume bestimmen durch seine senkrechte Projektion $P^{\prime}$ anf eine horizontale Ebene $\Pi$ (Vergleichsebene) und eine Höhenzahl (Kote), die seine Entfernung ron dieser Ebene angibt. Ter Zeichnung ist immer ein Maßstab bei- 
zufügen. und die Koten der unterhalb $\Pi$ liegenden Punkte sind mit negativem Vorzeichen zu verselien.

Eine gerade Linie ist hiernach bestimmt durch die Projektionen und die Koten ( = die kotierten Projektionen) von zwei ihrer Punkte.

Fig. 173.

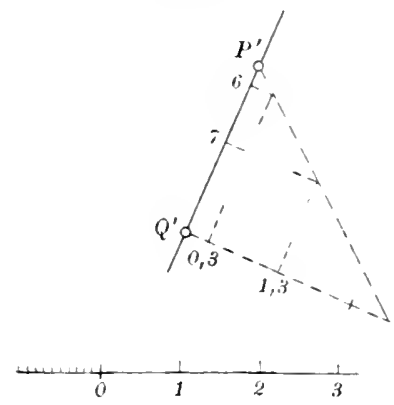

Sind auf der Projektion der Geraden die Punkte mit ganzzahligen Koten angegeben, so sagen wir, die Gerade sei graduiert. Der Abstand der Projektionen zweier Punkte der Gerarlen, deren Kotendifferenz $=1$ ist, heibt das Intervall der Geraden.

Un die Terbindungslinie der Punkte $P(5,7)$ und $Q(8,5)$ zu graduieren, konstruieren wir die Lmlegung der Geraden in die durch $P^{\prime}$ gehende Horizontalebene und die Umlegungen der Punkte, deren Entfermungen von dieser Ebene $=0,3$ und 1,3 sind (Fig. 173).

186. Zur Darstellung einer Ebene genïgt die Angabe einer graduierten Fallinie (Gefällema bstab, durch eine Doppellinie bezeichnet. rgl. Fig. 176). Bedentet $i$ das Intervall der Fallinie, so ist das Gefälle

$$
\begin{aligned}
& \text { Fig. 17t. } \\
& \text { ○ } B^{\prime} \\
& { }_{\circ} C^{\prime}
\end{aligned}
$$

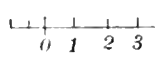$$
{ }^{\circ}{ }^{\prime}
$$
(die Böschung) der Ebene, d. h. die Tangente ihres Neigungswinkels gegen $\Pi=\frac{1}{i}$.

A ufgaben über die Ebene. a) Den Gefällemaßstab einer Ebenezu konstruieren, diedurch die Punkte $A(11,3), B(17,6)$ und $C^{\prime}$ $(15,4)$ bestimmt ist (Fig. 174). Wir graduieren die Terbindungslinie vou iırend zwei der gegrebenen Punkte, z. B. die Cierade $A B$, und ermitteln auf ihr den Punlit $D$, der dieselbe Kote hat wie C." Dann ist CIJ

Fig. 17 B.
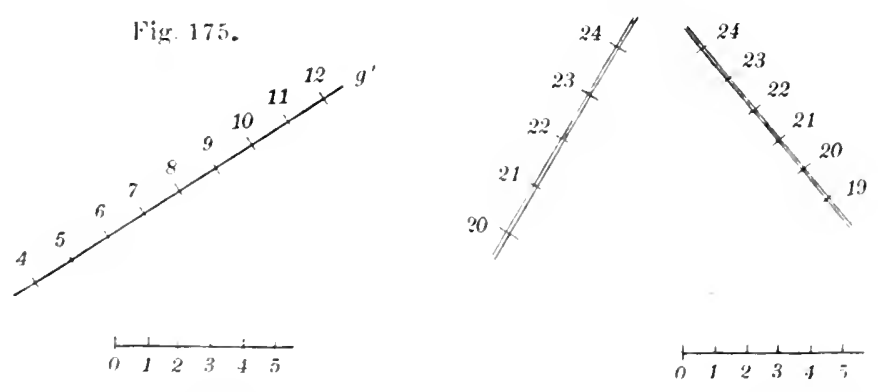

eine Hauptlinie der Ebene, (ler Gefallemalstab al:o $\left.\perp C^{\prime} I\right)^{\prime}$. Im seine Einteilung zu erhalten, ziehen wir durch die Pnnkte der graduierten Geraden $A^{\prime} P^{\prime}$ Parallelen zu $\left.C^{\prime} I\right)^{\prime}$. 
b) Wurch die Geradeg eine Ehene von gegebenem Gefälle, z. B. 3: 2, zu legen (Fig. 175). Konstruieren wir für irgend einen Punkt von $g$, z. B. für 11, den dem gegebenen Gefalle entsprechenden Böschungskegel (Art. 37), so schneidet dieser die Horizontalebene durch

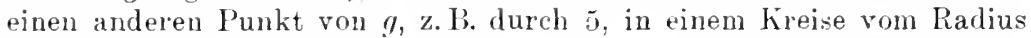
2 3 $(11-5)=4$. Die durch den Punkt 5 gehende Hauptlinie der gesuchten Ebene ist eine Tangente dieses Kreises usw.

c) Um die Schnittlinie zweier Ebenen zu konstruieren, ermitteln wir die Schnittpunkte zweier Paare von Hauptlinien mit gleichen Koten (Fig. 176).

187. Unter einer topographischen Fläche (Geländefläche) verstehen wir einen begrenzten Teil rer Erdoberfläche, der so klein angenommen wird, daß die Richtungen der Schwerkraft in den einzelnen

Fig. 177

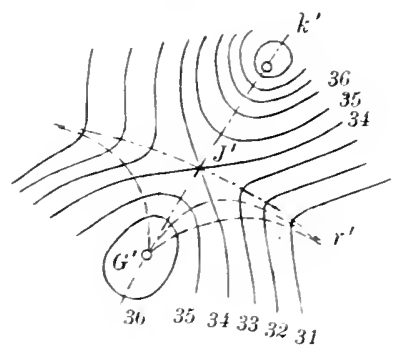

Fig. 178.

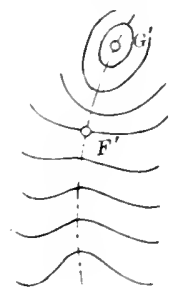

Flächenpunkten als parallel gelten können. Denken wir uns die Meeresoberfläche unter dem Festlande fortgesetzt, so dürfen wir das Stück des erweiterten Meeresspiegels, das unter dem so begrenzten Gelände liegt, als eine horizontale Ebene ansehen. Wir bestimmen dann die Punkte der topographischen Fläche durch ihre kotierten Projektionen in bezug auf diese Ebene.

Nehmen wir, von der Vergleichsebene ausgehend, in gleichen Alständen - etwa von 1 in - eine Reihe von Horizontalebenen (Schichtebenen) an, so schneiden diese die topographische Fläche in sogenannten Schichtlinien (Niveau- oder Horizontallinien, Höhenkurven, Isohypen), dereu Horizontalprojektionen und Koten zur Darstellung der Fläche dienen (Fig. 177). Jede Schichtlinie wird im Gelände durch Vermessen einer Reihe vou Punkten ermittelt, und dabei wird angenommen, daß sie zwischen je zwei der so gefundenen Punkte immer stetig verläuft. Es wird ferner vorausgesetzt, daß die Fläche zwischen zwei aufeinanderfolgenden Schichtlinien keine wesentlichen Gestaltsänderuugen aufweist; sie ist demnach nur angenähert und nicht gesetzmäßig bestimmt.

Die Schichtlinien sind stets geschlossene Furren.

Bei einer topographischen Fläche treten als ausgezeichnet die Punkte mit horizontaler Berührungsebene hervor. Sie heifen Gipfel- bzw. Ifuldenpunkte, wenn sie höher oder tiefer liegen als 
alle benachbarten Flachenpunkte, so dab sie von dell umgebenden Schichtlinien rings umschlossen werden. (In Figr.177 ist $G$ ein Gipfelpunkt.) Wenn dagegen das Gelände von einem solchen Punkte aus nach zwei Seiten ansteigt, wảhrend es nach zwei anderen Seiten abfällt. $\therefore$ sprechen wir von einem Joch-oder sattelpunkte (Punkt $J$ der Fidur). El ist also die tiefste Stolle zwischen zwei Bergrücken oder Kuppen und zugleich die Ausgangsstelle zweier 'Täler; die zuggehörige Schichtlinie hat in ihm eiuen Knotenpunkt. Darum gehört er zu den ly yerbolischen Punkten der Fläche, während es sich bei den Gipfelund Muldenpunkten un elliptisclie Punkte handelt (Art.137). Ausnahmsweise können in einem Punkte mit horizontaler Berührungsebene auch mehr als zwei hücken und ebenso viele Täler entspringen, und dann hat die Schichtlinie an dieser stelle einen mehrfachen Punkt.

Gehen wir auf der Fläche von einem (iipfelpunkte abwärts, so treffen wir zunächst auf Schichtlinien, die ihre hohle Seite überall der. Erdmasse zukehren. Dann kann aber an irgend einer Stelle eine Einbuchtung eintreten, so daß die hohle Seite nach außen gewendet wird, und dadurch entsteht ein sich abwärts ziehendes Tal (Fig. 17s). Den Ühergang zwischen beiden Arten von Schichtlinien bildet eine Kurve mit einem Flachpunkte $F$ (Art. 71 ). Taler entspringen also nicht blob in sattelpunkten.

188. Durch jeden Punkt einer topographischen Fläche geht eine Fallinie oder Linie gröbter. Neigung, welche die Schichtlinien überall rechtwinklig schneidet (vgl. die gestrichelten Linien in Fig. 177). Die Fallinien sind augenähert die Bahnen des auf der Fläche herabHiebenden Wassers, doch nur in Anfang seiner Bewegung, wenn es noch keine erbebliche liewegungsenergie lesitzt und durch das Be-

Fig. 179.

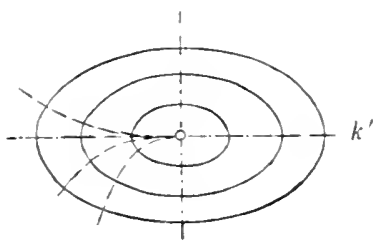

harrungsvermögen aus der auf den Schichtlinien sonkrechteu Richtung erst wenig abgelenkt wirl.

In jedem Gipfel-oder Muldenpunkte treffen unendlich viele Fallinien zusammen. Ilas ist sufort ersichtlich, wenn die topographische Fläche an der betrachteten Stelle zufällig den Charakter einer Indrehungsflache mit vertikaler Acbse lat. l)ann sind nämlich die vächsten Schichtlinien Parallelkreise der L'mdrehungsflache; der Gipfelpunkt sendet daher nach allen lichtungen Fallivien ans, die im Anfang wie Heridiane verlaufen. In allgemeinen sind aber die Schichtlinien in der unmittelharen Lmgebung eines Gij,felpunktes nicht kreisförmigr; sie haben vielmehr nahezu die Gestalt ähnlicher und ähnlich lieøender Ellipsen, deren Mittelpunkte sich anf der Vertikalen durch den (iipfelpunkt befinden (Fig. 179). Fieht man anf einer solchen Ellipse von einem Punkte ans, der zwischen den Scheiteln liegt, und konstruiert die anfwärts gerichtete Fallinie, so zeint sich. raß diese Kurve sich der groben Achse beständig nahert und sie im (iipfelpunkte beriuhrt: eine Ausnahme billet nur die Fallinie, die in der lichtung der kleinen Achse rom Gipfelpunkte abwärts läuft. Die ausgezeichnete Fallinie $k$, die durch die Endpunkte 
der großen Ellipsenachsen, also durch die Punkte stärkster Krïmmung der Schichtlinien geht, und der die benachbarten Fallinien an steigend tortwährend zustreben, heilt Kamm-oder Rückenlinie; sie lıat die Bedentung einer Wasserscheide.

Wird durch Fig. 179 ein Mnldenpunkt dargestellt, so nähern sich der Kurve $l_{i}$ die abwärts gerichteten Fallinien; in ihr sammelt sich das von den Hängen herabfliebende Wasser zu einem Bache. Wir bezeichnen sie dann als Rinnelinie oder Talweg.

Durch jeden Sattelpunkt gehen zwei Fallinien, nämlich eine Kammlinie $k$ und eine Rimnelinie $r$ (Fig. 177). Die eine verbindet ihn mit zwei benachbarten Gipfelpunkten, die andere mit zwei Muldenpunkten.

189. Aufgaben über topographische Flächeo. a) Um die Kote eines zwischen zwei s'chichtlinien liegenden l'unktes $P$ zu ermitteln, zeichnen wir den Schnitt (das Profil) der Fläche mit einer durch $P$ gelegten Vertikalebene in seiner Cmklappung in die Vergleichsebene oder in eine passend gewählte Schichtebene (Fig. 180). Wir errichten also in den schnittpunkten der einzelnen Schichtlinien mit der Spur der Profilebene Lote zu dieser Geraden, tragen auf ihnen die Höhen der betreffenden Schnittpunkte ab nnd verbinden die so erhaltenen Punkte durch eine Kurve. Dann bestimmt das in $P^{\prime}$ Fig. 180.

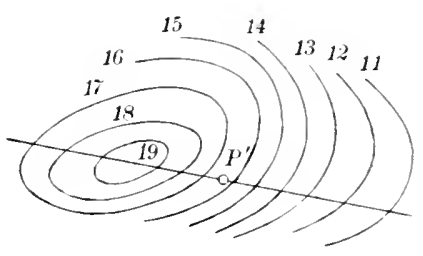
zur Spur errichtete Lot die Höhe des Punktes $P$ (graphische Interpolation). Soll der Schichtenplan von Konstruktionslinien möglichst frei gehalten werden, so klappen wir das Profil nicht um, sondern legen es an irgend eine andere Stelle der Zeichenebene.

Da das Profil, mit Ausnahme der höchsten und tiefsten Pnnkte, $z$ wischen zwei aufeinanderfolgenden Schichtlinien nahezu geradlinig verläuft, so genügt es in den meisten Fällen, die Profilebene nur mit den beiden dem Punkte $P$ benachbarten Schichtlinien zu schneiden und darauf die Höhe des Punktes unter der Annahme zn konstruieren, daß er der geraden Verbindungslinie der beiden Schnittpunkte angehört. Eiq anderes Näherungsverfahren besteht darin, daß wir durch den Punkt $P^{\prime}$ eine in zehn gleiche Teile geteilte Strecke (einen Maßstab) legen, so daß ihre Endpunkte auf die benachbarten Schichtlinien fallen, und an ihr die Höhe des Punktes $P$ schätzungsweise ablesen.

Dieselben Methoden dienen auch zur Lösung der umgekehrten Aufgabe, in einer durch ihre Spur gegebenen Profilebene die Flächenpunkte mit gegebenen Koten zu ermitteln. Auf diese Weise können wir feruer zwischen zwei anfeinanderfolgenden Schichtlinien noch andere von gegebener Höhe einschalten.

b) Das Gefälle der Flächeim Punkte $P$ wird durch das Gefälle seiner Berührungsebene gemessen. Um dieses zu bestimmen, legen wir durch $P$ senkrecht zur Tangente seiner (gegebenen oder durch Interpolation gefundenen) Schichtlinie eine Profilebene, deren Schnitt mit der 
Flache wie vorher durch Umklappen bestimmt wird. Ziehen wir dann in umgeklippten Punkte $P$ an die umgeklappte Schnittkurve eine Tangente, so ist diese eine Fallinie der Berührungsebene; sie bildet also mit der Spur der Profilebene den gesuchten Böschungswinkel.

c) Auf einem durch Schichtlinien dargestellten Gelände ron einem Punkte A einer Schichtlinie aus einen Weg von gegebenem koustanten Gefälle anzulegen. In Fig. 181 ist die Schichthöbe $=5 \mathrm{~m}$; soll also das Gefille des Weges z. B. = 1:10 sein, so ist die Horizontalprojektion des konstanten Wegstückes, das zwischen zwei Schichtlinien liegt, $=50 \mathrm{~m}$. Beschreiben wir mit dieser Strecke (in dem der Figur zugrunde liegenden Mabstab) von $A$ aus einen Kreis-

Fig. 181.

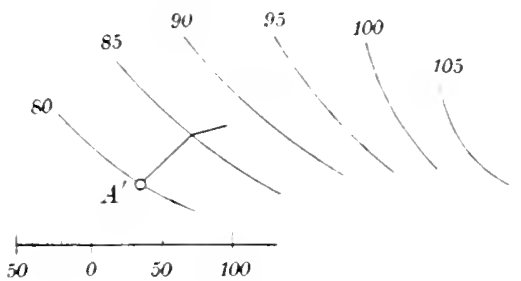

Fig. 18?.

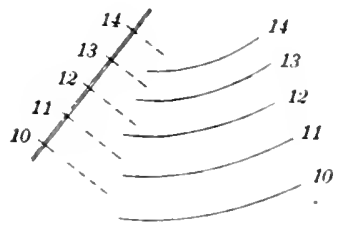

bogen bis zur nachsten Schichtlinie und gehen in derselben Weise ron einer Schichtlinie zur anderen weiter, so liefern die so erhaltenen Punkte die Projektion des gesuchten Weges.

d) Ver Schnitt einer Geländefläche mit einer beliebigen Ebene wird gefunden, indem man die Schichtlinien der Fläche mit den gleich kotierten Hauptlinien der Ebene schneidet und die erhaltenen Schnittpunkte miteinander rerbindet (Fig. 182). Ebenso konstruiert man die Schnittkure des Geländes mit einer krummen Fläche, deren schichtlinien bekannt sind.

190. Fonstruktion der Schnittlinien der Böschungsflächen einer horizontalen Strabe mit einem durch Schichtlinien gegebenen Gelände (Fig. 183). Iie Straße soll $60 \mathrm{~m}$ über dem Meeresspiegel liegen: ihre Ränder werden durch gerade Linien und Kreisbögren gebildet. Da sie die Schichtlinie 60 in zwei Punkten $A$ und $B$ schneiden, so ist die straße ron da an nach rechts durch $\mathrm{Ab}$ tragen des Erdreichs, nach links durch Aufschütten herzustellen. Die Böschung der erforderlichen Einschnitte und Dämme möge $2: 3$ betragen. Die zugehörigen Böschungstlächen sind Ebenen und gerade Kreiskegel; die Projektionen ihrer Schnitte mit den Schichtebenen des (ieländes sind also parallele Geraden und an diese anschließend konzentrische Kreisbögen in Abständen gleich der $1 \frac{1}{2}$ fachen Schichthöhe, d. b. $=1,5 \mathrm{~m}$. Wie Schnittlinien der Böschungsflächen mit dem Gelände werden dann wie im vorhergehenden Artikel unter $d$ gefunden.

Wir könneu diese Schnittlinien aber auch mit Hilfe von Querprofilen ermitteln, die wir senkrecht zu den Strabeurändern stelleu und in eine Horizontalebene, am einfachsten in die der Straße, umlegen. Jede Profilebene schneidet die Böschungsflächen in Fallinien und das 
Gelande in einer Kurve, deren Umlegung wie in Art. 189 unter a) konstruiert wird. Die Schnittpunkte beider Linien liegen auf der gesuchten Schnittkurre. - In der Figur bedentet $q$ die Spur einer solchen Profilebene.

Bei einer ansteigenden Straße mit geradlinigen Rändern sind die Böschungsebenen der erforderlichen Dämme und Einschnitte und ihre Schnittlinien mit den Schichtebenen des Geländes in derselben

Fig. 18::.
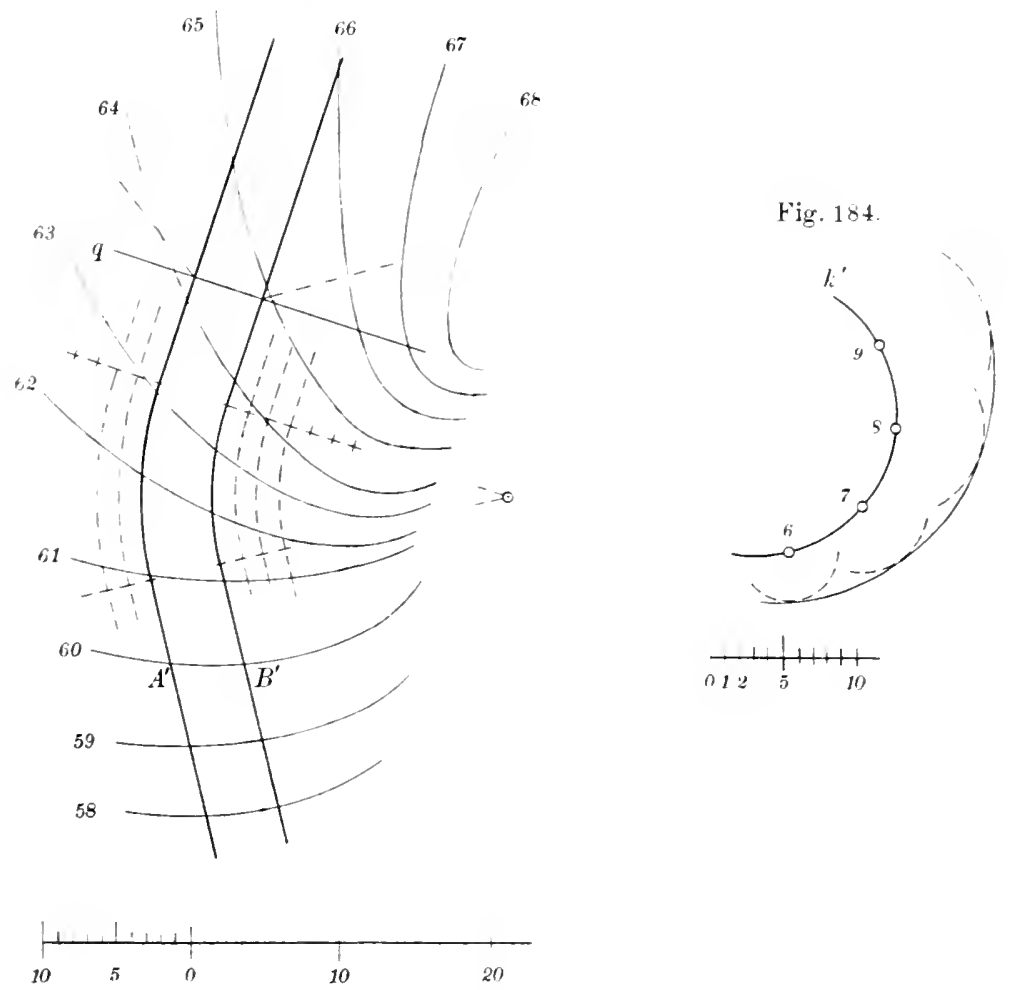

Weise zu konstruieren, wie in Art. 186 unter b). Verwendet man wieder Querprofile, deren Ebenen auf den Projektionen der Strabenränder senkrecht stehen, so schneiden diese die Böschungsebenen nicht mehr in Fallinieu. In der Praxis zeichuet man aber die Schnittlinien der Einfachheit wegen so, als ob sie Fallinien wären, weil der hierdurch begangene Fehler bei geringem Gefäll der Straße nicht erheblich ist.

Im allgemeinsten Falle, wenn die ansteigende Straße gekrümmte Ränder hat, können wir die Schichtlinien der Böschungsflächen in folgender Weise ermitteln. In Fig. 184 sei die Raumkurve $k$ durch ihre Horizontalprojektion $k^{\prime}$ und durch die Koten einer Reihe vou Punkten gegeben. Um durch $k$ eine Böschungsfläche $\Phi$ zu legen, deren Gefäll $=2: 3$ ist, konstruieren wir für die einzelnen Punkte der Kurve die 
zugehörigen Böschungskegel; dann ist $\Phi$ die einhüllende Fläche der simtlichen Kegelflächen. Nun schneiden die durch die Punkte $6,7,8 \ldots$ srelegten Kegel z. B. die Schichtebene 4 in Kreisen mit den Radien

$$
\begin{aligned}
& (i-4) \cdot \frac{3}{2}=3 \\
& (7-4) \cdot \frac{3}{2}=4.5 \\
& (8-4) \cdot \frac{3}{2}=6
\end{aligned}
$$

Die Einhüllende dieser Kreise ist die Schnittlinie der Fläche $\Phi$ mit der Schichtebene 4.

\section{Axonometrie.}

191. In technischen Zeichnungeu haudelt es sich in der Regrel um die Abbildung von Körpern, deren Kanten vorzugsweise drei aufeinander senkrechte Richtungen haben. Bej einer solchen Darstellung in Grund-. Aufriß und Seitenriß wählt man die Projektionsebenen $\Pi_{1}, \Pi_{2}, \Pi_{3}$ gewöhnlich so, dab ihre Schnittlinien $O \mathrm{X}, O Y, O Z$ jenen Hauptrichtungen parallel sind. Diese Anordunng gestattet ein hequemes Abgreifen der Maße, hat aber andererseits den Nachteil, daß die hierdurch erhaltenen Bilder wenig anschaulich sind, weil die auf einer Projektionsebene senkrechten Kanten und Seitenflächen des Körpers als Punkte, bzw. als Gierarten abgebildet werden. Um in orthogonaler Darstellung ein anschauliches Bild zu gewinnen, empfiehlt sich daher die Einführung einer vierten Projektionstafel $\Pi$, die auf keiner der Irei Hauptrichtungen senkrecht steht ${ }^{1}$ ).

Betrachten wir $O X, O Y, O Y$ als Achsen eines rechtwinkligen Koordinatensystems, so finden wir die Projektion $\bar{P}$ irgend eines P'unktes $P$ auf die Ebene $\Pi$ durch Abbildung seines aus den Strecken $O P_{x}=x, P_{x} P^{\prime}=\mathfrak{\eta}, I^{\prime} P=$ j bestehenden Koordinatenzuges. Diese Konstruktion läßt sich ohne weiteres ausfülıen, sobald zweierlei bekannt ist, nämlich: 1. die Projektionen der Koordinatenachsen und 2. für jede Achsenrichtung das zugehörige Verkürzungsverhältuis. 1)asselbe gilt auf Grund der in A.t. 2 abgelejteten Sätze, wenn wir den Körper nicht senkrecht, sondern schief auf $\Pi$ projizieren, nur dürfen wir dann nicht mehr vom "Verkürzungsverhältnis" sprechen, das jeder der Achsen znkommt, sondern allgemeiner vom "Projektionsverhältnis", da bei schiefer Projektion der Quotient zwischen Bild- und Originalstrecke auch größuer sein kann als 1.

1) Vgl anch Art. 12 und die Anmerkung zu Art. 33 ïber die Tran:formation le Projektionsebenen. Fin anderes Mittel zur Ableitung anschaulicher Bilder besteht in einer Lagenänderung des Körpers, hervorgebracht durch zwei aufeinanderfolgende brehungen um zwei Achsen, von denen di eine $\perp \Pi_{1}$, die andere $\perp \Pi_{2}$ ist. Bei der ersten Drehung andert der CirundriB nur seine Iage, aber nicht die Gestalt, während sich die Punkto im Aufriß in Parallelen $\%$ a verschieben, und das Ungekehrte gilt für die zweite Irehung. 
Das Terfahren, die Parallelprojektion einer Raumfigur a us den loordinaten ihrer Punkte zu konstruieren, wird als Axonometrie bezeichnet. Je nachdem die projizierenden Strahlen auf der Bildebene $\Pi$ senkrecht stehen oder nicht, unterscheiden wir senkrechte und schiefe Axonometrie.

Der Bildpunkt $\bar{P}$ heibt die axonometrische Projektion, das Bild $I^{\prime}$ ron $I^{\prime}$ der axouometrische Grundrib des Punktes $I^{\prime}$. Durch Angabevon $\bar{P}$ und $\Gamma^{\prime}$ ist der Originalpunkt $P$ bestimmt.

\section{Senkreclite Axonometrie.}

192. Die Lage des Koordinatensystems gegen die Bildebene $\Pi$ ist bestimmt, wenu wir die Spurpunkte $A, B$, $C$ der Koordinatenachsen $O X, O Y, O Y$ angeben und noch linzufügen, auf welcher Seite von $\Pi \pi$ der Anfangspunkt $O$ sich befinden soll. Dann ist nämlich $O$ der Schnittpunkt dreier Halbkugeln mit den Durchmessern $A B, B C, C A$.

Das Spurendreieck $A B C$ hat inmer drei spitze Winkel. Beweis. In jedem spitzwinkligen Dreieck ist das Quadrat jecler Seite kleiner als die Summe der Quadrate der beiclen anderen Seiten. Nun ist $A B^{2}=O A^{2}+O B^{2}$, $B C^{2}=O B^{2}+O C^{2}, C A^{2}=O C^{2}+O A^{2}$, daraus folgt aber $A B^{2}<B C^{2}+C A^{2}$ usw.

Figr. 185 .

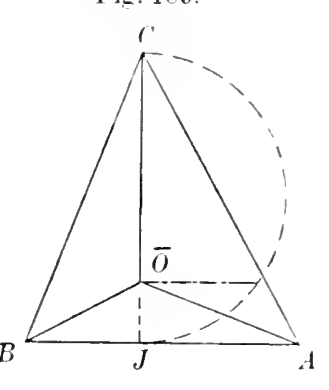

Aufgabe. Gegeben das Spurendreieck $A B C$, gesucht die Achsenprojektionen und die Achsenverkürzungsverhältnisse (Fig. 185). Die Gerade $O C$ steht senklecht anf der Ebene $O A B$, mithin steht ihre senkrechte Projektion $\bar{O} C$ auch senkrecht anf der Spur $A B$ der Ebene, d. h. die Achsenprojektionen sind die Höhenlinien des Spurendreiecks. - Um den zweiten Teil der Aufgabe zu lösen, ermitteln wir die Neigungswinkel $\alpha, \beta, \gamma$ der Geraden $O A$, $O P, O C$ gegen die Ebene $\Pi$ : Die projizierende Ebene ron $O C$ schneidet die Ebene $A O B$ in der Geraden $O . J \perp A B$. Legen wir das rechtwinklige Dreieck $C O J$, das $\bar{O}$ zum Höhenfußpunkt hat, in die Bildebene um, so gelangt $O$ nach $O_{0}$ auf dem Halbkreis über $C J$, und damn ist $\angle O_{0} C J=\gamma$. Jetzt finden wir die Winkel $\alpha$ und $\beta$, indem wir die rechtwinkligen Dreiecke $O O A$ und $O \bar{O} B$ in wahrer Größe zeichnen. Machen wir auf $\bar{O} C$ die Strecken $\bar{O} A_{0}=\bar{O} A$ und $\bar{O} B_{0}=\bar{O} B$, so ist $-O_{0} A_{0} \bar{O}=\alpha$ und $-O_{0} B_{0} \bar{O}=\beta$. Den Achsen $O X, O Y, O \%$ entsprechen demnach die Verkürzungsverhältnisse

$$
\lambda=\cos \alpha=\frac{\bar{O} A_{0}}{O_{0} A_{0}}, \quad \mu=\cos \beta=\frac{\bar{O} B_{0}}{O_{0} B_{0}}, \quad \nu=\cos \gamma={ }_{O_{0} C}^{\bar{O} C} .
$$

Nach dieser Vorbereitung erhalten wir die Projektion $P$ eine: durch seine Koordinaten $\{, \mathfrak{y}, \mathfrak{\jmath}$ gegebenen Punktes $P$ durch Abbildung der gebrochenen Linie $O P_{x} P^{\prime} P$. Wir machen also auf $\bar{O} A$ die Strecke 
$O I_{x}^{\prime}=\lambda x$ und ziehen $\| \overline{O B}$ alie strecke $I_{x}^{\prime} I^{\prime}=\mu \eta$, sowie $\| O C$ die strecke $I^{\prime \prime} I^{\prime}=\nu^{\prime}$. Dabei finden wir $\%$. B. die länge $\lambda \lambda^{\prime}$ durch eine einfache ('peration mit dem Handzirkel: Wir tragen $q$ von $O_{0}$ aus anf () $A_{0}$ ab und schlagen von dem so erhaltenen l'unkte einen lireis, der $O_{0} \bar{O}$ berührt.

193. Statt des Spurendreiecks könueu aneh die Aclisenprojektionen numittelbar gegeben werden, d. h. drei ron oinem l'nukte ausgehende Geraden, die drei stumpe Winkel einschlieben. Ham kann man jedes Dreieck, das jene Geraden zu Höhenlinien hat, als spurendreieck benutzen; unbestimmt bleibt also nur die Entfernung des Koordinatenanfangspunkts von der Bildebene, aber das ist ohne Einflul auf das Bild des darzustellenden Körpers, weil sich das Bild nicht ändert, wenn man das Koordinatensystem und den damit verbundenen Körper senkrecht zu $\Pi$ verschielst.

Un gut wirkende Bilder zu erhalten, wählt man die Aehsenprojektionen so, daß die Aehse $O Z$, die den vertikalen Kanten des Körpers parallel ist, auch im Bilde aufrecht steht, unl daß sie am schwächsten verkürzt wird, also mit der Ebene $\Pi$ den kleiusten Winkel einschliebt. Dies wird erreicht, wemn von den drei stumpfen Winkeln, die die Achsenprojektionen bilden, der Winkel $X \bar{O} \bar{Y}$ der größte ist wäre er nämlich ein gestreckter, die beiden andern Winkel also rechte; so wäre die $z$-Achse $\| \Pi$ und ihr Verkürzungsverhältnis $=1$. Man wird ferner die beiden andern Winkel möglichst ungleich wählen; dann gibt das Bild annähernd den Eindruck wieder, Jen man empfängt. wenn man den Körper aus großer Entfernung entweder mehr von vorn oder mehr von der Seite, jedenfalls aber bei geringer Neigung der Sebstrahlen gegen die horizontalen Flächen von oben oder von unten betrachtet.

194. Axonometrische Darstellung eines durch Grundund Aufrif bestimmten Hauses bei gegebenen Achsenpro-

Fig. 186 .

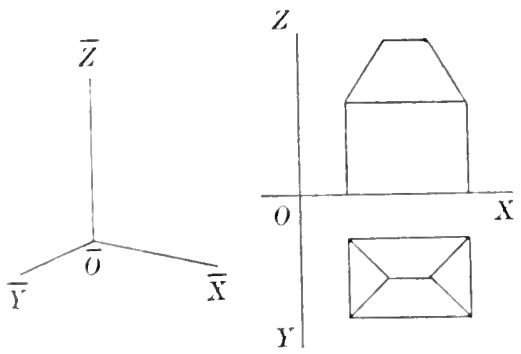
jektionen (Fig. 186). Wir zeichnen zmnächst ein Spurendreieck und ermitteln darauf die Verkürzungsverhältnisse wie in Art. 192 . Koustruieren wir dann den vollstäudigen axonometrischen Grundrib und errichten zuletzt alle Höhen, so wird der Grundrib vom Bilde des Hauses verleckt. Dieser Ühelstand läßt sich vermeiden, wenn wir jede einzelne Höhe um ein konstantes Stuck vergröbern, d. h, die Grundyißebene um dasselbe Stück parallel nach unten verschieben. Da übrigens das Haus symmetrisch ist in bezug auf die durch den First $\| \Pi_{2}$ selegte Ebene, so empfiehlt es sich, im vorliegenden Falle statt mit dem Grundriß, mit diesem Mittelschnitt zu beginnen und durch die so erhaltenen Punkte die n $O Y$ parallelen Kanten zu ziehen. 
Ist der gegebene Grund- und Aufrib eine flüchtig gezeichnete Handskizze mit eingeschriebenen Mabzahlen, so ist es vorteilhaft, fül. jede Achse einen Verkürzungsmaßstab zu zeichnen. Zu dem Zwecke konstruieren wir die Projektionen $1, m, n$ einer beliebigen Strecke $k$, die wir auf $O X, O Y, O \%$ der Reihe nach abtragen. Dann ziehen wir in einer besonderen Figur vier parallele Geraden $w, x, y, z$, deren $A b$ stände von einem Punkte $S$ der Ebene sich wie $k: l: m: n$ verbalten. Auf $w$ wird der wahre Maßstab der Bildebene anfgetragen und durch Strahlen aus $S$ auf $x, y, z$ projiziert.

195. Darstellung eines geraden Kreiszylinders, dessen Achse $\| O Z$ ist. Wir denken uns wieder die Projektionen der Koordinatenachsen gegeben und das Spurendreieck $A B C$, sowie das Verkürzungsverhältnis der $z$-Achse in bekannter Weise ermittelt. Der Grundkreis des Zylinders projiziert sich als Ellipse. Ihre große Achse ist das Bild des zu $\Pi$, also zu $A B$ parallelen Kireisdurchmessers; sie ist also selbst || $A B$ und ebenso groß wie der Durchmesser. Die Länge der kleineu Halbachse ergibt sich, wenu wir den Halbmesser des Kreises im Verhältnis $\bar{O} . I: O_{0} J$ verkürzen (vgl. Art. 192). Die scheiubaren Umrißlinien berühren die Bildellipse in den Endpunkten der großen Achse.

196. Darstellung einer Kugel vom Mittelpunkt $Q$ und dem Radius $r$, sowie der drei zu deu Koordinatenebenen parallelen Hanptkreise bei gegebenen Achsenprojektionen $\bar{O} \bar{X} Y$. Wir bezeichnen mit $G, H, J$ bzw. die Schnittpunkte der Geraden $O \bar{O}, \bar{O} Y, \bar{O} Y$ mit den auf ihnen senkrechten Seiten $B C, C A$, $A B$ eines beliebigen Spurendreiecks, mit $O_{1}, O_{2}, O_{3}$ die Lagen, in die der Punkt " durch Umklappung der rechtwinkligen Dreiecke $A O G$. $B O H, C O . J$ in die Ebene $\Pi$ gelangt. Dabei sind die anf $A G, B H$, $\mathrm{C} J$ der Reilie nach senkrechten Strecken $\mathrm{OO}_{1}, \mathrm{OO}_{2}, \mathrm{OO}_{3}$ einander gleich und es ist $-O_{1} A G=\alpha,-O_{2} B H=\beta,-O_{3} C J=\gamma$.

Der scheinbare Umriß der Kugel ist das Bild ihres zu $\Pi$ parallelen Hanptkreises $u$, also der Freis $\overline{u m} \bar{Q}$ mit dem Radius $r$. Sind $Q L$, ( $M, Q N$ die zu $O X, O Y, O Z$ parallelen Kugelradien, so finden wir in bekannter Weise ilure Projektionen $\bar{Q} \bar{L}=r \cos \alpha, Q M=r \cos \beta$, () $N=r \cos \gamma$. Dann ist z. B. das Bild des durch $M$ und $N$ gehenden Hauptkreises eine Ellipse mit den konjugierten Halbmessern $\bar{Q} \bar{M}$ und $\bar{Q} \bar{N}$; sie beriihrt $\bar{u}$ in den Endpunkten ihrer großen Achse, die anf QI, senkrecht steht, und die kleine Halbachse ergibt sich durch Verkürzung von $r$ im Verbältnis $\sigma G: O_{1} G$.

Zum Vergleich zeichne man von den drei axonometrisch dargestellten Körpern anch ihre Bilder in schiefer Parallelprojektion (Art.3, Art. 90 und Fig. 96 a, sowie Art. 105). Dadurch zeigt sich, dab die senkrechte Axonometrie gefälliger wirkende Bilder liefert, namentlicl, wenu es sich um die Darstellung krummer Flächen handelt.

197. Die Gerade $O \bar{O}$ bildet mit den drei Koordinatenarhsen die Vinkel $90^{\circ}-\%$ nsw. Da die Summe der Quadrate der drei Rirhtungs- 
kosinus einer Geraden $=1$ ist, so ergibt sich

$$
\cos ^{2} \alpha+\cos ^{2} \beta+\cos ^{2} \gamma=2
$$

oder

$$
\lambda^{2}+\mu^{2}+\nu^{2}=2
$$

Hierbei ist jede der drei Zahlen $\lambda, \boldsymbol{\mu}, \boldsymbol{v} \leqq 1$. Wäre z. B. $\boldsymbol{v}=1$, also die $z$-Achse $\| \Pi$, so wïrden die Projektionen der beiden anderen Achsen zusammenfallen - eine Annahme, die überaus unschöne Bilder liefert, und die wir deshalb von der weiteren Betrachtung ansschließen (Übereckprojektion). Für $v^{\prime}<1$ folgrt aus 1 ), daß $\lambda^{2}+\mu^{2}>1$ ist, $m$ so mehr ist also

$$
\lambda^{2}+u^{2}>\nu^{2}
$$

Bezeichnen wir wieder mit $7, m, n$ drei Längen, die sich wie die Verkürzungsverhältnisse $\lambda, \mu, \nu$ verhalten, so gibt es immer eine Strecke $k$, die, auf den drei Achsen abgetragen, die Strecken $l, m, n$ als Projektionen liefert. Da

$$
\lambda=\frac{l}{k}, \quad \mu={ }_{k}^{m}, \quad \nu=\frac{n}{k} . . .
$$

ist, so erhalten wir zur Bestimmung von $k$ ans 1) die Gleichung

$$
2 k^{2}=l^{2}+m^{2}+n^{2} \text {. . . }
$$

Die Zahlen $l, m, n$ heißen Verhältniszahlen. Zwischen ihnen bestehen nach 2) drei Lngleichungen von der Form

$$
7^{2}+m^{2}>n^{2}
$$

d. h. die Summe der Quadrate zweier Verbältuiszahlen ist immer größer als das Quadrat der dritten.

188. Statt der Achsenprojektionen kann man anch die Verhältniszahlen vorschreiben, und zwar wählt man für diese gern drei ganze Zahlen; gute Bilder liefert z. B. die Annahme $7: m: n=9: 5: 10$. Dann entsteht als Lmkehrung der früher gelösten die Aufgabe: Gegeben die Verhältniszahlen $l, m, n$, gesucht die Verkürzungsverlältnisse und die Achsenprojektionen. Wir zeichnen, unter Zngrundelegung einer beliebigen Längeneinheit, drei Strecken ron deu Längen $l, m, n$, und konstruieren die Strecke $k$ nach Gleichung 4) als Kathete eines gleichschenklig rechtwinkligen Dreiecks mit der Hypotenuse $\sqrt{l^{2}+m^{2}+n^{2}}$. Hierauf machen wir in einer nenen Figur auf einer beliebigen Geraden $S T$ die Strecken $S U=7, S V=m, S W=n$, beschreiben $1 \mathrm{~m} S$ mit dem Radius $k$ einen Kreisbogen und bestimmen seine Schnitpunkte $X, Y, Z$ mit den Loten, die in $U, V, W$ zu $S T$ errichtet sind. Dann ist $-X S T=\alpha,-Y S T=\beta, \angle Z S T=\gamma$. Machen wir daher die Strecke $S T \perp S T$ gleich der willkiirlich gewählten Entfernung des Koordinatenanfangspunkts $O$ von $\Pi$ und ziehen durch $R$ zu $S T$ eine Parallele, die $S X, S Y, S Z$, sowie das in $S$ zu $S Z$ errichtete Lot bzw. in $I, E, F, G$ schneidet, so sind $I I), R E, R F$ gleich den Projektionen der zwischen () und $\Pi$ liegenden Achsenabschnitte, entspreclien alio den Strecken $\bar{O} A, O B, \overline{O C}$ in Fig. 1S5, und $R G$ ist gleich der Projektion $\vec{O} J$ des Lotes von () anf $A B$. Konstruieren wir 
lemnach in einer dritten Figur über der Kathete $\bar{O} J=R G$ nach entgegengesetzten Seiten die rechtwinkligen Dreiecke $\bar{O} J A$ und $\bar{O} J B$ mit den Hypotenusen $\bar{O} A=R D, \bar{O} B=R E$ und verlängern $\bar{O} J$ über $\bar{O}$ bis $C$ um die Strecke $R F$, so sind $\bar{O} A, \bar{O} B$, $O C$ die Projektionen und A. $B, C$ die Spuren der $x-, y-, z$-Achse.

199. Nach der Beschaffenheit der Verhältniszahlen unterscheiden wir drei Arten von senkrechter Axonometrie:

1. isometrische Projektion, wenn alle drei Verhältniszahlen einander gleich sind;

2. dimetrische Projektion, mit zwei gleichen Verhältniszahlen:

3. trimetrische Projektion, wenn alle drei Verhältniszahlen roneinander verschieden sind.

Bei der isometrischen Projektion haben die Koordinatenachsen gegen $\Pi$ dieselbe Lage, wie die von einer Ecke ausgehenden Kanten eines Würfels, wenn die durch denselben Punkt gezogene Diagonale $\perp \Pi$ ist. Dann bilden die Projektionen der Ecken, die nicht auf der Diagonale liegen, ein regelmäßiges Sechseck, und die Achsenprojektionen schneirlen sich unter Winkeln von je $120^{\circ}$. Für die Verkürzungsverhältnisse folgt aus 1) der leicht konstruierbare Wert

$$
\lambda=\mu=v=\sqrt{\frac{2}{3}} \text {. }
$$

In der Regel zeichnet man aber in diesem Falle alle Koordinaten in wahrer Gröbe, also ein vergröbertes Bild des Körpers.

Hierdurch ergibt sich z. B. als Bild eines zur $x y$-Ebene parallelen Kreises eine Ellipse $k$, deren zu $\bar{O} \mathrm{X}$ und $\bar{O} Y$ parallele Durchmesser $E F$ und $G H$ einander konjugiert und dem Kreisdurchmesser gleich sind (Fig. 187). Lie Achsen $A B$ und $C D$ von $k$ liegen in den Diagonalen $Q R$ und $S T$ des von den Tangenten in $E, F, G, H$ gebildeten Rhombus. L'm ihre Längen zu ermitteln, betrachten wir $k$ als die perspektiv affine Figur zu dem Kreise $l_{0}$ über dem noch unbekannten Durchmesser $A B$ mit $Q R$ als Affinitätsachse. Dann entsprechen den gleichen konjugierten Durchmessern EF und $G I I$ von $k$ in $k_{0}$ die Halbierungslinien $E_{0} F_{0}$ Fig. 187. und $G_{0} H_{0}$ der rechten Winkel zwischen $Q R$

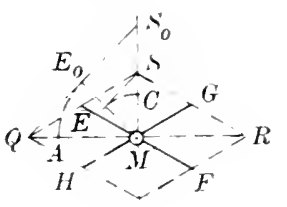
und $S T$, der Ellipsentangente $Q S$ mithin die Kreistangente $Q S_{0}$ unter einem Winkel von $45^{\circ}$ gegen $Q R$. Demnach ist $E_{0}$ der Fuppunkt des vom Ellipsenmittelpunkt $M$ auf $Q S_{0}$ gefällten Lotes, und hieraus ergibt sich $M A=M E_{0}$. Ebenso finden wir die Länge der Halbachse $M C$ als Kathete eines gleichschenklig rechtwinkligen Dreiecks mit der Hypotenuse $M S$.

\section{Schiefe Axonometrie.}

200. Wird nicht mehr gefordert, daß die projizierenden Strahlen auf der Bildebene senkrecht stehen, und lassen wir ihre Richtung zunáchst noch unbestimmt, so können wir sowohl die Achsenprojektionen 
als auch die Verhältniszalıen willkürlich annehmen: es gilt nämlich der Satz ron Pohlke: Drei Strecken von beliebigen Längen und Richtungen, die in einer Ebene von einem Punkte ausgehen. können immer als die Projektionen dreier gleich langen, in einem Punkte rechtwinklig zusammenstobenden Strecken angesehen werden. Die drei Strecken dïrfen aber nicht in eine einzige Gerade fallen, es darf auch höchstens eine von ihnen gleich Null sein.

Um diesen Satz zu beweisen, lösen wir \%uvor die Aufgabe: Ein gerades dreiseitiges Prisma durcheine Ebeneso zu schneiden, daß die entstehende Schnittfigur einem gegebeneu Dreieck ähnlich wird (Fig. 18s). In der Zeichenebene $\Pi$ sei $A B C$ die Grund-

Fig. 18R.

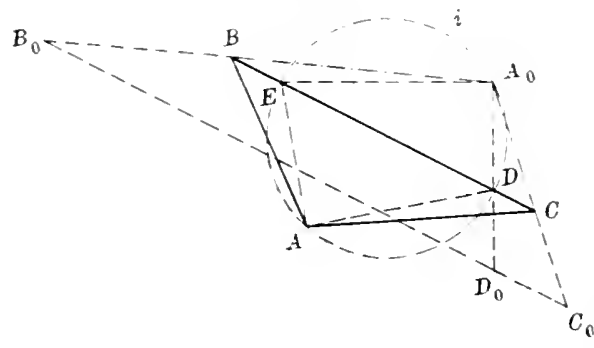
fläche des Prismas, $A_{0} B C$ das Dreieck, dem die Schnittfigur ähnlich werden soll. Wir legen die gesuchte Ebene $\Sigma$ der Einfachheit wegen durch $A$ und bezeichnen mit $A B_{1} C_{1}$ die entstehende Schnittfigur, init $D$ den Schnittpunkt ron $B C$ und $B_{1} C_{1}$. - Nach Art. 30 und 41 befindet sich in der $C_{0}$ Ebene $\Sigma$ ein einziger rechter Winkel mit dem Scheitel $A$, dessen senkrechte Projektion auf $\Pi$ wieder ein rechter ist, nämlich der Winkel zwischen der Spur $A D$ und der durch $A$ gehenden Falllinie. Ist $E_{1}$ der Schnittpunkt dieser Fallinie mit $B_{1} C_{1}$ und $E$ seine senkrechte Projektion auf $\Pi$, so verhält sich

$$
C D: D E: E B=C_{1} D: D E_{1}: E_{1} B_{1},
$$

d. h. den Punkten $D$ und $E_{1}$ der Schnittfigur $A P_{1} C_{1}$ entsprechen in der dazu ähulichen Figur $A_{0} B C$ die Punkte $D$ und $E$. Den Geraden $A D$ und $A E_{1}$ der ersten Figur sind also die Geraden $A_{0} D$ und $A_{0} E$ der zweiten zugeordnet, mithin ist auch $-D A_{0} E$ ein Rechter. Die Punkte $I$ und $E$ liegen daher auf dem durch $A$ und $A_{0}$ gehenden Kreise i. dessen Mittelpunkt sich auf $B C$ befindet.

Hierlurch ist zunächst der Schuittpunkt $I$ ) von $B C$ mit der Spur ron $\Sigma$ und damit diese selbst bestimmt. Unter den beiden Schnittpunkten ron $i$ und $I ; C$ haben wir nämlich denjenigen mit $D$ zu hezeichnen, für welchen der spitze Winkel $D A_{0} C$ größer ist als seine senkrechte Projektion $D A C$ (Art. 41$)$.

Nachen wir auf $A_{0} D$ die Strecke $A_{0} D_{0}=A D$ und ziehen durch $D_{0}$ die Gerade $B_{0} C_{0} \| B C$ bis $A_{0} B$ und $A_{0} C$, so wird durch das Dreieck $A_{0} B_{0} C_{0}^{\prime}$ die Schnittfigur in wahrer Gröle dargestellt. Damit ist die Entfermung des Punktes $B_{1}$ von .1 gefunden, also die Ebene $\Sigma$ (zweideutig) bestimint.

201. Beweis des Pohlkeschen Satzes (Fig. 189). Sind $Q L$, $Q M, Q N$ ilrei beliebige Strecken in der Ebene $\Pi$ und $O \mathrm{X}, O Y, 0 \%$ Irei beliebige, aber gleich lange Strecken im Raume, rie paarweise auf- 
einander senkrecht stehen, so brauchen wir nur zu zeigen, dah wir von der Figur OX Y'Z eine Parallelprojektion konstruieren können. die zur

Fig. 189 .

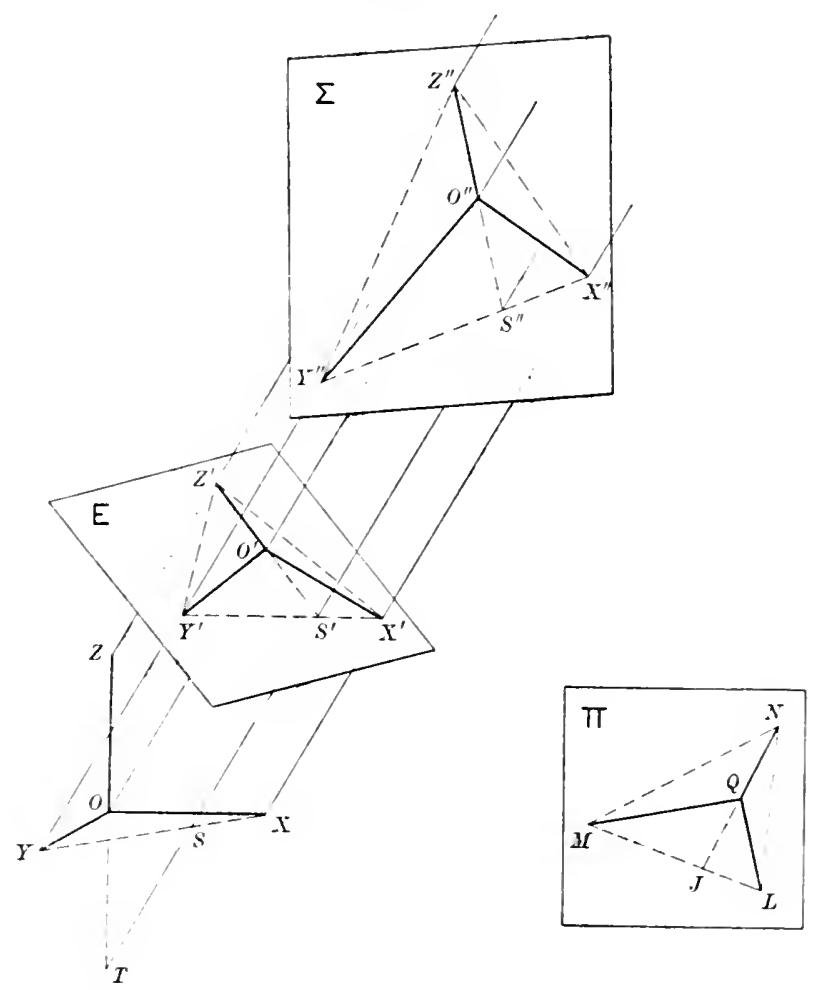

Figur QLMN ähnlich int. Zu dem Zwecke schneiden wir $L M$ und (2) $N$ in $J$ und bestimmen auf $X Y$ und $O \%$ bzw. die Punkte $S$ und $T$ घemäß den Proportionen

$$
\mathrm{X} S: Y S=I . J: M . J
$$

und

$$
\text { o } T: Z T=Q J: N J \text {. }
$$

Projizieren wir die Ramnfigur in der Richtung $S T$ auf eine dazu senkrechte Ebene $E$, so erhalten wir von $S$ und $T$ eine gemeinsame Projektion $S^{\prime}$ und in der Bildfigur $O^{\prime} X^{\prime} Y^{\prime} S^{\prime}$ sind die Punktgruppen

$$
\begin{gathered}
X^{\prime} S^{\prime} Y^{\prime} \sim \text { L.J II } \\
Z^{\prime} O^{\prime} S^{\prime} \sim \text { NQJ. }
\end{gathered}
$$

Nun können wir das gerade Prisma, das $X^{\prime} Y^{\prime} Z^{\prime}$ zur Grundfäche, also $X X^{\prime}, Y Y^{\prime}, Z Z^{\prime}$ zu Seitenkanten hat, nach der vorhergehenden Aufgabe mit zwei Scharen paralleler Ebenen in der Weise schneiden, dab die Schnittfigur dem Dreieck $L I I N$ ähnlich wird. Ist $\Sigma$ eine dieser 
Ebenen, $X^{\prime \prime} Y^{\prime \prime} Y^{\prime \prime}$ die entstehende Schnittfigur, und sind ()$^{\prime \prime}$ and $S^{\prime \prime}$ die Schnittpunkte von $\Sigma$ mit $O O^{\prime}$ und $S S^{\prime \prime}$, so wird auch

$$
X^{\prime \prime} S^{\prime \prime} Y^{\prime \prime} \sim I . I M
$$

nnd

$$
Z^{\prime \prime} O^{\prime \prime} S^{\prime \prime} \sim N Q . J,
$$

es ist also in der Tat die Figur $O^{\prime \prime} X^{\prime \prime} Y^{\prime \prime} Z^{\prime \prime} \sim Q I, M X$.

202. Bei den praktischen Anwendungen der schiefen Axonometrie legt man gewöhnlich die Bildebene $\Pi$ parallel zu einer der Koordinatenebenen, z. B. parallel zur vertikalen $x z$-Ebene (schiefe Paralle)perspektive oder liaralierperspektive). Dann erscheint der Winkel zwischen $x$ - und $z$-Achse im Bilde wieder als rechter, und sämtliche $x$ - und $z$-Koordinaten bleiben unveräudert. Für die Bilder der $y$-hoordinaten kann man (übereinstimmend mit dem Pohlkeschen Satze) die Richtung, sowie das Projektionsverhältnis belielig wählen: zeichnet man sie unter einem Winkel von $30^{\circ}$ gegen das Bild der $x$-Achse und anf die Hälfte verkürzt, so ergibt sich das von uns zur Herstellung von Skizzen von Anfang an benutzte Abbildungsverfaluen (rgl. Art. 3 bis 5 ).

Die schiefe Parallelperspektive bietet gegenüber der senkrechten Axonometrie den Vorteil, dab die zu einer Koordinatenebene parallelen Flächen in wahrer Gröbe dargestellt werden, olne daß die darauf senkrechten Kanten zu Punkten verkürzt erscheinen. Damit ist aber, wie ïberhaupt bei schiefer Parallelprojektion. der Nachteil rerbunden, dals man das Bild, um den richtigen Eindruck zu erhalten, schrägr von der Feite (streng genommen, ans unendlich rrober Entfermung) betrachten muß. Deshalb hat es auch wenig Zweck, bei Anwendung der schiefen Axonometrie die Bildebene geneigt gegen alle drei Achsen zu stellen, denu dadurch geht nur jener Torteil wieder verloren, den die der schiefen Parallelperspektive zugrunde liegende Annahme mit sich bringt, während der soeben herrorgehobene Nichteil jedenfalls bestehen bleibt. Man bedient sich dann besser der senkrechten Axonometrie, die schönere Biller liefert, weil bei ihr die Sehrichtung zur Bildtafel senkrecht steht. 


\section{Zweiter Absehnitt. \\ Die Zentralprojektion.}

\section{Theoretische (freie) Perspektive.}

Darstellung des Punktes, der Geraden und der Ebene.

203. Wir bezeichnen im folgenden mit $\pi$ die Bildebene, nit O das Projektionszentrum (Gesichtspunkt, Auge), nit $I I$ den Fubpunkt des Lotes von $O$ auf $\Pi$; rlie Gerade $O H$ heift der Hauptstrahl, $H$ der Hauptpunkt, die Länge $d$ rer Strecke $O H$ die Distanz des Bildes. Durch Hauptpunkt und Distanz ist die Lage des Projektionszentrums bestimmt, weln anßerdem festgesetzt wird, daß das Lot $H O=d$ immer nach vorn, d. ll. nach der Seite des Beschauer's, errichtet werden soll.

Die Zentralprojektion einer Figur wird auch deren Perspektive gellannt.

204. Die Projektion des Punktes $P$, also der Schnittpunkt ron OP mit $\Pi$, soll mit $P_{c}$ bezeichnet werden. Durch Angabe von $P_{c}$ ist der Originalpunkt noch nicht bestimmt.

Jeder Punkt von $\Pi$ ist sein eigenes Bild. Das Bild eines unendlich fernen, nicht in $\Pi$ liegenden Punktes ist ein endlicher Punkt. Tedem Punkte der Ebene $\Pi^{v}$, die durch $O \| \Pi$ gelegt wird, entspricht ein unendlich fermer Bildpunkt. Wir nennen $\Pi^{v}$ die Verschwindungsebene. Die hinter $\Pi^{v}$ liegenden Punkte werden von dem der Ebene $\Pi$ zugewendeten Auge nicht gesehen, deshalb bezeichnen wir ihre Bilder als virtuell. Dagegen entsprechen den Punkten, die sich zwischen $\Pi^{v}$ und $\Pi$, oder hinter $\Pi$ befinden, reelle Bildpunkte.

205. Die Projektion jeder nicht durch $O$ gehenden Geraden $g$ ist wieder eine Gerade, nämlich die Schnittlinie $g_{c}$ der projizierenden Ebene $O g$ mit $\Pi$ (Fig. 190). Geht die Originalgerade durch $O$, so ist ihre Projektion ein Punkt.

Die Geraden $g$ und $g_{c}$ schneiden sich im Spurpunkte $G$. Dem unendlich fernen Punkte $G^{\infty}$ der Originalgeraden 1$)$ entspricht auf $g_{c}$ der Schnittpunkt $G_{c}^{\infty}$ des Parallelstrahles von $g$, d. h. der Parallelen durch $O \mathrm{zu} g$, mit der Ebene $\Pi$. Der Punkt $G_{c}^{\infty}$, also das Bild des

I) Tgl. Anmerkung auf S. 11. 
unendlich fernen l'unktes del Geraden y, heibt der liluchtpunkt ron 9. - Die Bilder paralleler. Geradengehen durch einengemeinsamen Fluchtpunkt.

Durch Spur- und Eluchtpunkt ist die Originalgerade bestimmt. - Ein Punkt im Raume ist bestimnt durch sein Bild

Fig. 190

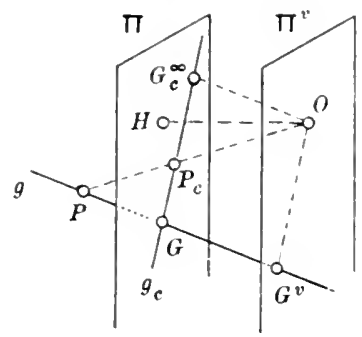

und durch Spur-und Fluchtpunkt einer durch ihn gehenden Geraden.

Schneidet $g$ die Ebene $\Pi^{v}$ in $(; "$, so entsteht das Parallelogramm $O G_{c}^{\infty} G_{i} G^{v}$, also ist $g_{c} \| O\left(^{r}\right.$. Der Punkt $G^{r}$, dem der uuendlich ferne l'unkt ron $g_{c}$ entspricht, heißt der Verschwindungspunkt von $y$. Originalgeraden, die sich in $\Pi^{2}$ scbueiden, haben parallele Bildel.

Sonderfälle. a) Ist die Gerade $g \| \Pi$. so ist $g_{c} \| g$. Dann verbalten sich die $A b-$ schnitte auf $g_{c}$ wie die entsprechenden Abschnitte a uf $g$. In diesem Falle ist zur Festlegung von $g$ aluel der Geraden $y_{c}$ noch irgend ein Punkt von $g$ erforderlich, der seinerseits wieder in der vorher angegebenen Weise bestimmt wird.

b) Der Hauptpunkt $H$ ist der Fluchtpunkt aller Normalen zu $\Pi$.

Wir bezeichnen die Parallelen zu $\Pi$ im folgenden kurz als Frontlinien, die Normalen zu $\Pi$ als 'Tiefenlinien.

206. Eine nicht durch $O$ gehende Ebene $\mathrm{E}$ wird bestimmt durch irgend zwei ihrer Geraden, am zweckmäbigsten durch ihre Spurlinie e

Fig. 191.

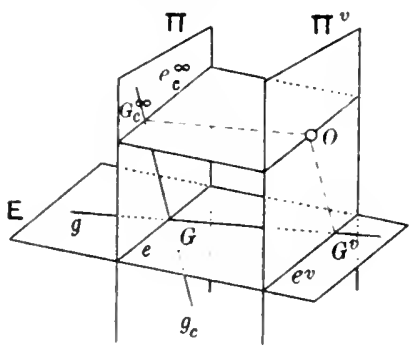

und ihre unendlich ferne Gerade $e^{\infty}$, die selbst wieder durch ihr Bild $e_{c}^{\infty}$, d. h. die Schnittlinie von $\Pi$ mit der Parallelebene durch () zu E gegeben ist(Fig. 191). Die Crerade $e_{c}^{\infty}$ lieibt die Fluchtlinie von $E_{;}$sie ist $\| e$. - Parallele Ebenen Laben dieselbe Fluchtlinie.

Die Ebene $E$ schneidet $\Pi^{r}$ in ihrer Verschwindungslinie $e^{v} \| e$.

Sonderfälle. a) Gelit E durch "), so fallen $e$ und $e_{c}^{\alpha}$ zusammen, und die Bilder aller Punkte von $E$ liegen auf $e$.

b) Ist $E \| \Pi$, so sind $r$ und $e_{r}^{\infty}$ unendlich fern; eine solche Front ebene wird be-timmt durch Angabe eines ihrer Punkte. Dann entspricht jeder in $E$ liegenden Figur ein ihr ähnliches Bild.

c) Ist $\mathrm{E} \perp \pi$, so geht $e_{c}^{\infty}$ durch $H$.

207. Liegt eine Gerade in einer Ebene, so liegen Sirur-. Flucht-und Terschwindungspunkt der Geraden bzw. in der Spur-, Flucht-und Verschwindungslinie der Ebene.

Daraus folgt: Wenn zwei Geraden einander schneiden, so ist die Terbindungslinie ihrer Spurpunkte parallel zur. Ferbinduneslinie ihrer Fluchtunukte. 
Abbildung einer ebenen Figur.

208. Konstruieren wir von einer in der Ebene $E$ liegenden Figur $A B C \ldots$ die Zentralprojektion $A_{c} B_{c} C_{c} \ldots$, so stehen Original- und Bildfigur in folgender Beziehung: Alle Terlindungslinien entsprechender Punkte gehen durch einen Punkt. nämlich lurch das Projektions\%entrum 0 , und alle Schnittpunkte entsprechender Geraden liegen auf einerGeraden, nämlich auf derSpurliuie $e$ der Originalebene (Fig. 192).

Solche Figuren lieißen kollinear in perspektiver Lage (perspektiv kollinear): $O$ ist das Kollineationszentrum, $e$ die Kollineationsachse,

Fig. 192.

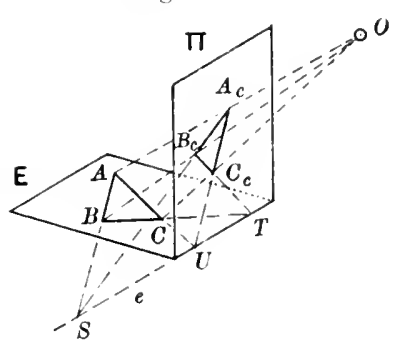
$A A_{c}$ ein Kollineationsstrahl. Nach Aufhebung der perspektiven Lage bezeichnen wir die Figuren einfach als kollinear.

Die in Art. 49 behandelte Verwandtschaft der Affinität ist ein sonderfall der Kollineation.

209. Konstruktion des Bildes einer ebenen Figur. Das Projektionszentrum $O$ sei gegeben durch den Hauptpunkt $H$ und die Distanz $d$, die Originalfigur $₹$ lurch die Spur $e$ und die Fluchtlinie $e_{c}^{x}$ ihrer Ebene $E$ und durch ihre Umlegung $\tilde{F}_{0}$ um r in $\Pi$, wobei noch hinzugefügt werden mub, welcher Teil von $E$ sich hinter der Ebene $\pi$ befindet (Fig. 193). Ist $g_{0}$ irgend eine Fierade von $\oiint_{0}$, so kennen wir von der Bildgeraden $g_{c}$ zunächst den Spurpunkt $G=e \times g_{0}$. Der zugehörige Fluchtpunkt $G_{c}^{\infty}$ ist der Schnittpunkt von $e_{t:}^{\infty}$ mit der Parallelen durch $O$ zu $g$. Um ihn zu ermitteln, drehen wir die Ebene $O e_{c}^{\infty}$ um $e_{c}^{\infty}$ in demselben Sinne wie $E$, bis sie mit $\Pi$ zusammenfällt. Bezeichnen wir mit $O_{0}$ die neue Lage von $O$, mit $J$ den Schnittpunkt von $H O_{0}$ und $e_{c}^{x}$, so ist $H O_{0} \perp e_{c}^{\infty}$ und $J O_{0}=J O$, d. h. gleich der Hypotenuse $J O^{0}$ eines rechtwinkligen Dreiecks

Fig. 193.

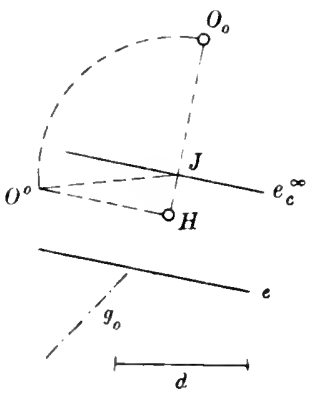

$H J O^{\prime \prime}$ mit den Katheten $H J$ und $H O^{\circ}=d$. Daun erhalten wir als Umlegung von $O G_{c}^{\infty}$ die Parallele durch $O_{0}$ zu $g_{0}$ und damit den Punkt $G_{c}^{\infty}$, also schließlich $g_{c}=G G_{c}^{\infty}$.

Der Punkt $J$ ist der Fluchtpunkt aller Geraden von $E$, die auf der Spurlinie senkrecht stehen (Hauptfluchtpunkt der Ebene).

210. Im zu irgend einem Punkte $P_{0}$ der umgelegten Originalebene das Bild $I_{c}$ zu konstruieren, ziehen wir durch ihn irgend zwei Geraden $q_{0}$ und $r_{0}$ als Umlegungen zweier Geraden $q$ und $r$ von $E$ und bestimmen $\mathrm{zu}$ ihnen in der eben entwickelten Weise die Bilder $q_{c}$ und $r_{c}$; dann ist $P_{c}=q_{c} \times r_{c}$. Benutzen wir dabei als $q_{0}$ die Verbindungslinie von $P_{0}$ und $O_{0}$, so fällt $q_{c}$ mit $q_{0}$ zusammen, d. h. $O_{0} P_{0}$ ist eine 
selbstentprechende Gerado und enthailt deshalb auch den Punkt I'. $^{\prime}$ Daraus folgt der satz: Legt man eiue ebene Figur um die spurlinie ihrer Ebene und das Projektionszentrum in demselben sinne um die zugehörige Fluchtlinie in die Bildebene nm, so sind Bild und Cmlegung der Originalfigur perspektiv kollinear mit dem umgelegten Projektionszentrum als Kollineationszentrum und der Spurlinie der Originalebene als hollineationsachse.

Man konstruiere liernach das Bild eines in E liegenden und durch seine Umlegung gegebenen Quadrats.

211. Schneiden wir $E$ mit der Terschwindungsebene $\Pi^{r}$ in der Verscluwindungslinie $c^{*}$, so ist nach Fig. 191 Abstand $e^{v} e=\mathrm{Ab}$ stand $O e_{c}^{\infty}$; wir erhalten daher die Umlegung $e_{0}^{*}$ von $e^{v}$, indem wir im Abstande $J O_{0}$ zn $e$ eine Parallele zielren. (Es ist also die Mitte des Abstands $O_{0} e$ auch die Mitte zwischen $e_{0}^{v}$ und $e_{c}^{\infty}$.)

Der Schnittpunkt von $g_{0}$ mit $e_{0}^{v}$ ist der umgelegte Verschwindungspunkt $G_{0}^{v}$ von $g$. Da der zugehörige Bildpunkt $G_{c}^{v}$ auf $g_{c}$ unendlich fern liegt, so ist nach Art. $210 \quad O_{0} G_{0}^{v} \| g_{c^{*}}$. Hieraus ergibt sich eine zweite Konstruktion der Bildgeraden $g_{c}$ mittels der Punkte $G$ und $G_{0}^{v}$.

212. Anf Grund der perspektiv kollinearen Beziehung zwischen einer ebenen Figur und ihrer Zentralprojektion können wir die früher

Fig. 194 .

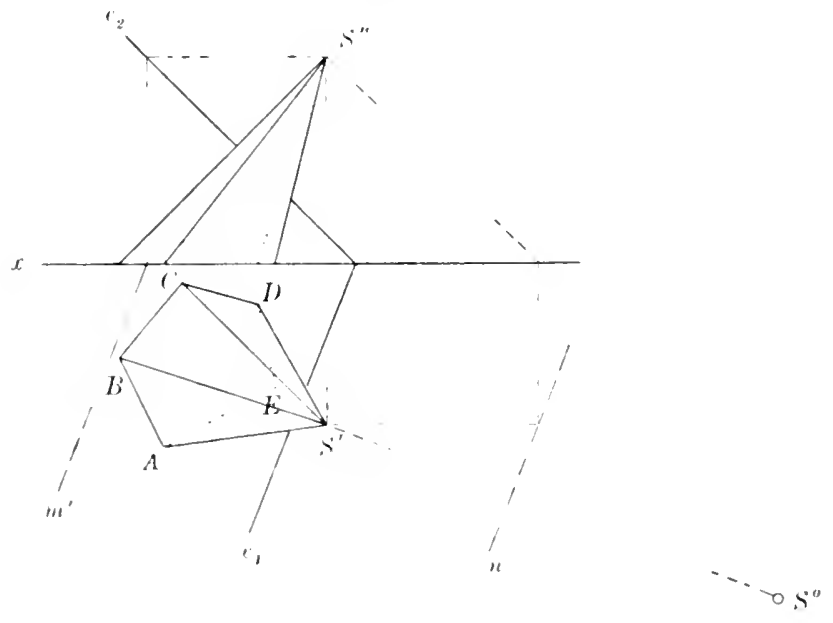

gelösten Aufgaben über die ebenen Schnitte der Pyramiden und Kegelflächen noch in anderer Weise behandeln. Denn der Schnitt der in Fig. $19+$ (früher in Fig. (i2) in Grund- und Aufrib dargestellten I'yramide mit der Ebene $E\left(\rho_{1} e_{2}\right)$ ist die perspektiv kollineare Figur $A_{1} B_{1} \ldots$ zu der in $\Pi_{1}$ liegenden Grumlfläche $A B \ldots$ mit ler Spitze $S$ als Kollineationszentrum und $e_{1}$ als Kollineationsachse; dabei entspricht der unendlich fernen Geraden rou $\Pi_{1}$ in $E$ die Schnittlinie $m$ mit rer 
durch $S$ gehenden Horizontalebene und der unendlich fernen Geraden von $E$ in $\Pi_{1}$ die Grundribspur $n$ der Ebene durcl $S \| E$. Im Grundrib seht also die Gerade $A_{1}^{\prime} B_{1}^{\prime}$ durch die Schnitpunkte von $A B$ mit $e_{1}$ and der Parallelen durch $S^{\prime}$ zu $A B$ mit der Geraden $m^{\prime}$ (vgl. den Schluf des Art. 58).

Die wahre Größe der Schnittfigur ergibt sich durch Lmlegung der $E$ in die $\Pi_{1}$. Bezeichnen wir mit $S^{0}$ die entsprechende Cmlegung von $S$ um die Gerade $n$, so ist die wahre Größe $A_{1}^{0} B_{1}^{0} \ldots$ perspektiv kollinear zu $A B \ldots$ mit $S^{0}$ als Kollineationszentrum und $e_{1}$ als Kollineationsachse; die Gerade $A_{1}^{0} B_{1}^{0}$ geht daher durch den Punkt $A B \times e_{1}$ parallel zur Verbindungslinie von $S^{0}$ mit dem Punkte $A B \times n$.

213. Die Teilungspunkte der Geraden. In Fig. 195 ist die Gerade $g$ durch ibr Bild $g_{c}=G G_{c}^{\infty}$ und das Projektionszentrum $)$ wie immer durch $I$ und $d$ gegeben. Auf der Bildgeraden $g_{c}$ soll vom Punkte $P_{c}$ aus - etwa in der Richtung von $G$ nach $P_{c}$ eine Strecke abgetragen werden, deren wahre Länge $=1 \mathrm{i}$ - $\mathrm{t}$.

Wir ziehen durch $G$ und $G_{c}^{x}$ in beliebiger Richtung zwei Parallelen $e$ und $\epsilon_{c}^{x}$ und betrachten sie als Spur- und Fluchtlinie einer durch $g$ gelegten Ebene E (rgl. die Skizze 195a). In E gibt es zwei Scharen paralleler Linien, die mit $g$ and $e$ gleiche Winkel einschlieben, also auf beiden Geraden gleiche Stücke abschneiden. Ihre Fluchtpunkte $O_{1}$ und $O_{2}$ liegen auf $e_{c}^{x}$, und zwar bildet der Strahl $0 O_{1}$ mit $O G_{c}^{\infty}$ und $e_{c}^{x_{c}}$ dieselben einander gleichen Winkel, wie die entsprechenden Parallelen mit den Geraden $g$ und $e$, mithin ist $O_{1} G_{c}^{\infty}=O G_{c}^{\infty}$. Die wahre Länge des Parallelstrabls $O G_{c}^{\infty}$ von $y$ ist aber $=O_{0} G_{c}^{x}$, wenn $O_{0}$ wie früher die Cmlegung ron O um $e_{c}^{x}$ bedeutet, oder sie ergibt sich Fig. 195 .

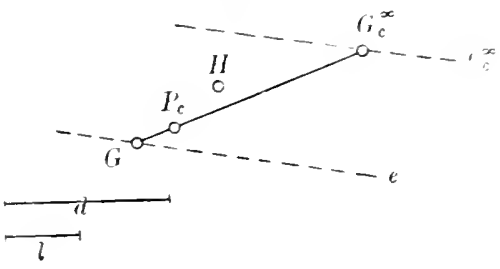

Fig. $195 \mathrm{id}$.

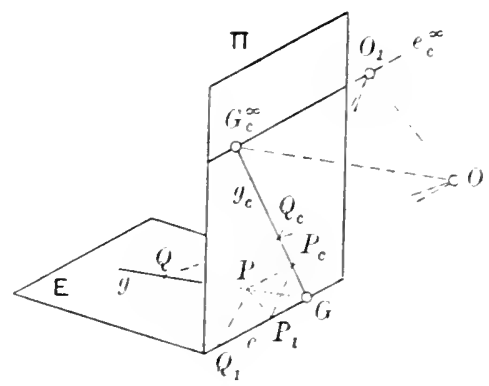

direkt als Hrpotenuse eines rechtwinkligen Dreiecks mit den Katheten $H G_{c}^{x}$ und $O H=d$. Dann erhalten wir $O_{1}$ und $O_{2}$, indem wir diese Länge von $G_{c}^{\infty}$ aus auf $e_{c}^{x}$ beiderseits abtragen. Ziehen wir nun die Gerade $O_{1} I_{c}^{\prime}$ bis zu ihrem Schnittpunkte $P_{1}$ mit $e$ und machen auf $e$ die Strecke $P_{1} Q_{1}=1$, so schneidet $O_{1} Q_{1}$ die Bildgerade $g_{c}$ im gesuchten Punkte $Q_{c}$, dessen wahre Entfernung von $P_{c}=l$ ist; denn $O_{1} P_{1}$ und $O_{1} Q_{1}$ sind die Bilder $z$ weier Geraden, die mit $g$ und $e$ gleiche Winkel einschließen, folglich sind $G P_{1}$ und $P_{1} Q_{1}$ die wahren Längen der Strecken $G P_{c}$ and $P_{c} Q$.

In derselben Weise können wir auch den Punkt $O_{2}$ verwenden. Dieses Verfahren erspart die Umlegung der Ebene E. 
Die l'unkte $O_{1}$ und $O_{2}$ heißen die Teilung-punkte oder II ljpunkte der Geraden ? hinsichtlich der Ebene E.

Aus unseren Darlegungen ergibt sich die wichtige Regel: Die Teilungspunkte der in der Ebene E liegenden Geraden! werden gefunden, indem man die Länge des Parallelstralils $U G_{c}^{\infty}$ rom Fluchtpunkte $G_{c}^{x}$ aus auf $e_{c}^{\infty}$ abträgt. Die Strahleu, die den Teilungspukt mit den Endpunkten einer auf $g_{c}$ liegenden Strecke verbinden, begrenzen auf der Spur e der Ebene die wahre Länge der Strecke.

214. Lin die strecke $P^{\prime} Q$ im Bilde in eine Anzahl, z. B. drei, gleicher' Teile zu teilen, ist die Konstruktion des Teilungs-

Fig. 196.

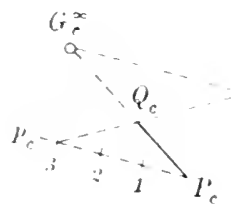
punkts nicht erforderlich (Fig. 196). Wir ziehen einfach durch den einen Endpunkt $P_{c}$ der Bildstrecke $P_{c} Q_{c}$ und durch ihren Fluchtpunkt $G_{c}^{x}$ in beliebiger Richtung zwei Parallelen $p_{c}$ und $e_{c}^{\infty}$, tragen auf $p_{c}$ eine beliebige Länge von $I_{c}^{\prime}$ aus dreimal ab, wodurch die Punkte 1, 2, 3 erhalten werden, scbneiden $e_{c}^{\infty}$ mit $3 Q_{c}$ im Punkte $F$ und bestimmen die Schnittpunkte $R_{c}$ und $S_{c}$ von $P_{c} Q_{c}$ bzw. mit $F 1$ und $F 2$. Betrachten wir nämlich $e_{c}^{x}$ als Fluchtlinie eiver durch $P Q$ gehenden Ebene, so ist $P_{c} 3$ das Bild einer in drei gleiche Teile geteilten Frontlinie dieser Ebene. und den Geraden $F 1, F 2, F 3$ entsprechen parallele Originalgeraden: also ist $P R=R S=S Q$.

215. Die reduzierten Punkte. Die bisher entwickelten Regelu bedürfen einer Ergänzung für den Fall, daß gewisse, zur Durchführung

Fig. 197 .

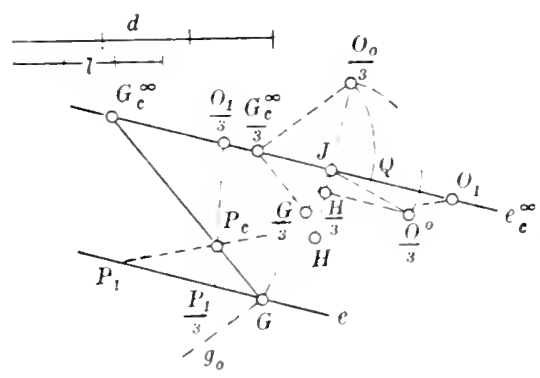
der Konstruktion notwendige Punkte außerhalb des Rahmens der Zeichenfläche liegen. Wir betrachten z. B. die Aufgabe: Ge geben ist das Projektionszentrum $O$ durch Hauptpunkt $H$ und Distanz $d$, eine Ebene $\mathbf{E}$ durch $e$ und $e_{c}^{\infty}$ und in ihr die Gerade $g$ durch ibre L'm]egung $g_{0}$; man soll die Bildgerade gc konstruieren und auf ihr vom Spurpunkte $G$ a us die Länge $l$ a btragen (Fig. 197). Wiein Art. 209 fällen wir zunächst von $H$ auf $\rho_{c}^{\infty}$ das Lot $H J$; auf diesem befindet sich das mugelegte Projektionszentrum $\sigma_{0}$. Ist nun die Distanz so grob, dab $O_{0}$ unerreichbar wird, so machen wir auf $H J$ die Strecke $J \frac{H}{n}=\frac{1}{n} J H$, ferner ${ }_{n, n}^{H O^{0}} \perp J H$ gleich dem "ten Teile der Distanz und auf rer Verlängerung von $I J J$ die Strecke ${ }^{O_{0}}=J \frac{0^{0}}{n}$; dabei ist die Zahl $n$ so grob zu wahlen, dab alle genannten Punkte innerhalb der Zeichenfläche liegen (in der Figur ist 
$n=3)$. Ziehen wir ditnn $O_{n} G_{r}^{\infty} \| g_{0}$ bis $e_{r}^{\infty}$ und machen auf $e_{c}^{\alpha_{r}}$ die Strecke $J G_{c}^{\infty}=n . J{ }_{n}^{G_{c}^{\infty}}$, so ist $G_{c}^{\infty}$ der Fluchtpunkt ron \%, also $g_{\text {. }}$ $=G G_{r}^{\infty}$.

Die Punkte $\frac{H}{n},{ }_{n}^{G_{c}^{\infty}}$... heiben reduzierter Hauptpunkt, reduzierter Fluchtpunkt usw.

Lim auf $y_{c}$ den Punkt $P_{c}$ zu ermitteln, dessen wahre Entferuung von $G=l$ ist, bestimmen wir auf $e_{c}^{x_{s}}$ den Teilungsjunkt $O_{1}$ von $g$ mittels $G_{c}^{x} O_{1}=n \cdot \frac{G_{c}^{\infty}}{n} \frac{O_{0}}{n}$, sowie auf $e$ den Punkt $P_{1}$ mittels $G P_{1}=l$ nnd ziehen $O_{1} P_{1}$. Iachen wir statt dessen anf $e_{c}^{\infty}$ und $e$ die strecken $G_{c}^{x} \frac{O_{1}}{n}=\frac{G_{c}^{\infty} O_{0}}{n}{ }_{n}$ und $G \frac{P_{1}}{n}=\frac{l}{n}$, so geht auch die Gerade $\frac{O_{1}}{n} \frac{P_{1}}{n}$ durch den gesuchten Punkt $P_{c}$; dabei ist $\frac{O_{1}}{n}$ der reduzierte Teilungspunt von $g$.

Wird auch der Fluchtpmnt $G_{c}^{\infty}$ unzugänglich, so finden wir die Bildgerade $g_{c}$ mit Hilfe des reduzierteu Spurpunkts: Machen wir auf $J G$ die Strecke $J \frac{G}{n}=\frac{1}{n} J G$, so ist $g_{c} \| \frac{G}{n} \frac{G_{c}^{\infty}}{n}$. Um in diesem Falle den Teilungspunkt $O_{1}$ zu ermitteln, machen wir auf $e_{c}^{\infty}$ in der Richtung nach $J$ die Strecke ${ }_{n}^{G_{c}^{x_{\infty}}} Q=\frac{G_{c}^{x}}{n} \frac{O_{0}}{n}$; daun ist $J O_{1}=n . J Q$. Befindet sich auch $O_{1}$ außerhalb der Zeichenfläche, so ermitteln wir den reduzierten Teilungspunkt $\frac{O_{1}}{n}$ zufolge der Gleichung:

$$
J \frac{O_{1}}{n}=J G_{c}^{x}-\frac{O_{1}}{n} G_{c}^{x}=n . J \frac{G_{c}^{x}}{n}-\frac{O_{0}}{n} \frac{G_{c}^{x}}{n} .
$$

Weitere Aufgaben über Geraden und Ebenen.

216. Wir beginnen mit einigen Aufgaben, die nur Lagenbeziehungen enthalten, dagegen von \a \& beziehungen völlig frei sind. \%u ihrer Lösung ist die Kenntnis des Projektionszentrums nicht erforderlich.

a) Die Sclinittlinie $g$ der Ebenen $\mathbf{E}\left(e, e_{c}^{x}\right)$ und $\boldsymbol{\Phi}\left(f, f_{c}^{\infty}\right)$ zu konstruiereu (Fig. 198). Die Spurlinien $e$ und $f$ treffen sich im Spurpunkte $G$ ron $g$; ebenso ist der Fluchtpunkt $G_{c}^{x}=e_{c}^{x} \times f_{c}^{x}$. - Ist nur einer dieser Punkte erreichbar, so schneide man die beiden Ebenen mit einer geeigneten Hilfsebene $\Sigma\left(s, s_{c}^{\infty}\right)$. Dann geht $g$ durch den Schnittpunkt der Geraden $E \times \Sigma$ und $\Phi \times \Sigma$ (vgl. hiermit Art. 27).

b) Den Schnittpunkt $P$ der Geraden $g\left(G, G_{c}^{\infty}\right)$ mit der Ebene $\mathbf{E}\left(e, e_{c}^{\infty}\right)$ zu konstruieren (Fig. 199). Man lege durch $g$ eine 
beliebige Hilfsebene $\Phi\left(f, f_{c}^{x}\right)$ und bestimme die Scbnittlinie $k$ von $E$ und $\Phi_{;}$dann ist $P=g \vee k$ (vgl. Art. 2s).

c) Durch den Punkt $P$ der Geraden $k\left(h, h_{c}^{\infty}\right) z u$ eine ${ }^{\circ}$ anderen Geraden $7\left(L, L_{c}^{x}\right.$ ) eine Parallele $m$ zu ziehen (Fig, 200).

Fig. 199.

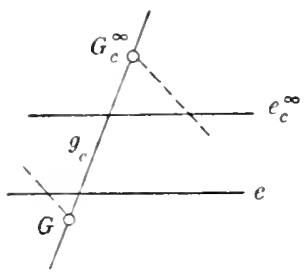

ler Fluclitpunkt ron $m$ fällt mit $L_{c}^{\infty}$ zusammen; also ist $m_{c}=P_{r} L_{c}^{\infty}$. Da ferner $k$ und $m$ sich schneiden, so liegt der Spurpunkt $M$ von $m$ auf der Parallele durch $K$ zu $K_{c}^{\infty} L_{c}^{\infty}$.

d) Die Verbindungslinie g der Punkte P' und $Q$ zu bestimmen, die bzw. auf den Geraden $k\left(K, K_{c}^{\infty}\right)$ und $l\left(L, L_{c}^{\infty}\right)$ gegeben sind (Fig. 201). Auf der Bildgerarien $g_{c}=P_{c} Q_{c}$ mu noch der Spurpunkt $G$, sowie der Fluchtpunkt $G_{c}^{2}$ ermittelt werden. Die Gerade $g$ liegt nun in der Ebene $\mathrm{E}=\mu l$, deren Spur- und Fluchtlinie wir konstruieren. indem wir durch $l^{\prime} \mathrm{zu} l$ die Parallele $m$ ziehen. Bestimmen

Fig. 201.
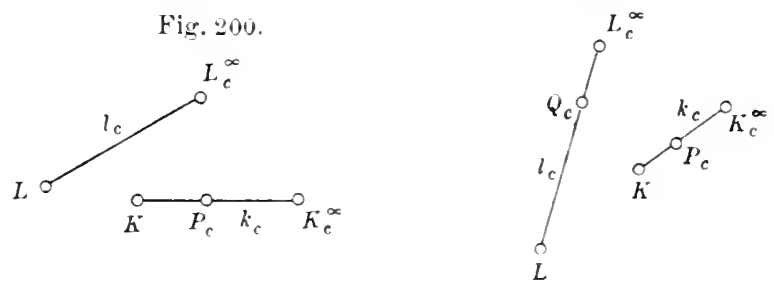

wir von $m$ den Spurpunkt $M$ wie in der vorigen Aufgrabe, so ist $\epsilon=L, M$. und $e_{c}^{\infty}$ geht durcl $L_{c}^{\infty} \| e$. Dann sind $G$ und $G_{c}^{\infty}$ die Schnittpunkte von $g_{r}$ bzw. mit $e$ und $e_{c}^{\infty}$.

217. Bei den folgenden Aufgaben metrischen Inhalts ist das Projektionszentrum $O$ immer lurch den Hauptpunkt $M$ und die Distanz $d$ gegeben.

a) Den Winkel $\gamma$ zweier (sich scluneidenden oder windschiefen) Geraden $a\left(A, A_{c}^{\alpha}\right)$ und $b\left(B, B_{c}^{\alpha}\right)$ \%u bestimmen (Fig. 20.2). Da auch die Parallelstrahlen $O A_{r}^{\infty}$ und $O B_{r}^{x}$ den Winkel $\gamma$ einschlieben, so finden wir seine wahre Größe durch L'mlegung des Dreiecks $A_{c}^{\alpha} O B_{c}^{*}$ in $\Pi$. Wir ziehen also $H J \perp A_{c}^{x} J_{c}^{x}$ und machen $J O_{0}$ gleich der Hypotenuse eines rechtwinkligen Dreiecks mit den Katheten II.J und d, so ist $-A_{c}^{\alpha} O_{0} B_{c}^{x}=\gamma$.

b) Im I'unkte I'der Ebene E $\left(e, e_{c}^{\infty}\right)$, desseu Bild $P_{c}$ gegeben ist, ein Lot ron der gegebenen Lälue $7 \mathrm{zu}$ errichten (Fig. 203). l)er gemeinsame Fluchtpunkt $\boldsymbol{N}_{c}^{\infty}$ aller Normalen zu $E$ ist rler Schnittpunkt von $\Pi$ mit dem Lote in $O$ zur Ebene $O e_{c}^{x}$. Die senkrechte Projaktion dieses Lotes auf $\Pi$ geht durch $H \perp e_{c}^{x}$ und trifft $e_{c}^{s}$ im Hauptlluchtpunkte $J$ ron $E$. Die Punkte $J, O, \lambda_{c}^{x}$ bilden demnach ein bei () rechtwinkliges Dreieck mit dem Höhenfußpunkte $I$, und wir erhalten 
den Punkt $N_{c}^{\infty}$, indem wir das Dreieck in seiner Cmlegung zeichnen: wir ziehen also $H O^{\circ} \perp K J$ und $=l$ und $O^{0} N_{c}^{\infty} \perp O^{0} J$ bis $H J$.

Die Gerade $l^{\prime} N^{x}$ ist das Bild der unbegrenzten Geraden $n$, die in $P$ auf $\mathrm{E}$ senkrecht steht. Un ihren Spurpunkt $N$ zu ermitteln, ziehen wir durch $P$ eine in $E$ liegende Gerarle, z. B. die Senkrechte zu e, die $J$ zum Fluchtpunkt, mithin den Schnittpunkt $K$ ron $I^{\prime} \cdot J$ mit zum Spurpunkt hat. Dann liegt $N$ auf der Parallele durch $K$ zu $J V_{c}^{\infty}$. - Die Geraden $K N$ und $J N_{c}^{\infty}$ sind Spur- und Fluchtlinie der durch $n \perp \Pi$ gelegten Ebene.

Jetzt wird auf $n$ die Länge $l$ nach der Methode res Teilungspunkt: aufgetragen: Wir machen auf $J N_{c}^{\infty}$ die Strecke $N_{c}^{\infty} O_{1}=N_{c}^{\alpha} O$, d. h.

Fig. 203.

Fig. 202.
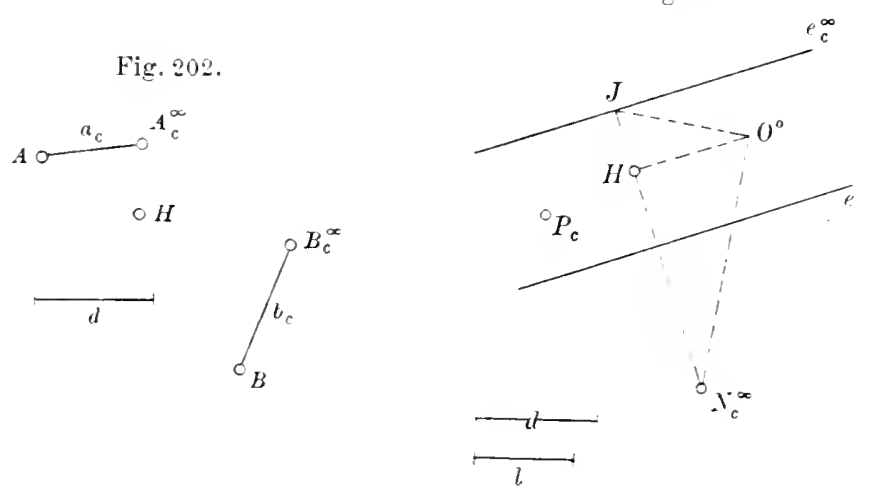

$=N_{c}^{\infty} O^{0}$, projizieren $P_{c}$ aus $O_{1}$ nach $P_{1}$ auf $K N$ und tragen die Länge $l$ ron $P_{1}$ aus (in dem einen oder dem entgegengesetzten Sinne) auf $K N$ ab. Ziehen wir von dem so erbaltenen Punkte eine Gerade nach $O_{1}$, die $n_{c}$ in $Q_{c}$ schneidet, so ist $P_{c} Q_{c}$ das Bild des gesuchten Lotes.

Die Lösung dieser Aufgabe gestattet in Verbindung mit Art. 210 die Darstellung eines geraden Prismas ron gegebener Grundfläche und Seitenkante.

\section{D) arstellung des Kreises.}

218. Die Zentralprojektion des Kreises ist der Schnitt eines hreiskegels mit der Bildebene, also eine Ellipse (oder ein Kreis), eine Parabel oder Hyperbel, je nachdem der Kreis mit der Verschwindungslinie seiner Ebene keinen, einen oder zwei Punkte gemein bat.

Aufgabe. Die Zentralprojektion $l_{c}$ des Kreises $k$ zu koustruieren, der durch seine Ebene $E\left(e, e_{c}^{\infty}\right)$ und seine Umleorung $k_{0}$ um $e$ in $\Pi$ gegeben ist (Fig. 204). Wir ziehen zunäch-t $H . J \perp e_{c}^{x}$ und bestimmen in bekannter Weise die Cmlegung $O_{0}$ des Projektionszentrums $O$ um $e_{c}^{\infty}$ (Art. 209), sowie die umgelegte Verschwindungslinie $e_{v}^{\imath}$ von $E$ (Art. 211 ). 
Erster Fall: $k_{0}$ schneidet $e_{0}^{v}$ nicht; $k_{c}$ ist also eine Ellipss. Sei $M_{0}$ der Mittelpunkt von $l_{0}, A_{0} B_{0}$ der auf $e$ senkrechte Durchnesser, $S$ sein Schnittpunkt mit $e$, so entspechen den Punkten $A_{0}$ und $B_{0}$ in der perspektiv kollinearen Beziehung zwischen $k_{0}$ und $l_{c}$ die Schnitt-

Fig. 204.
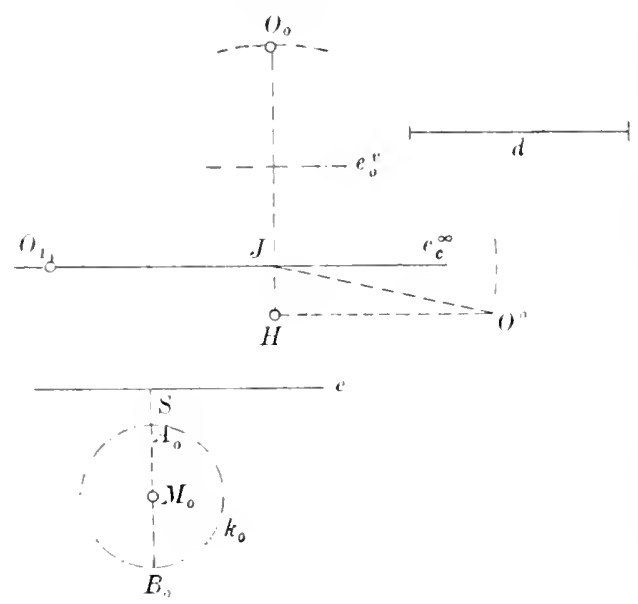

punkte $A_{c}$ und $B_{c}$ von $S . J$ mit $O_{0} A_{0}$ und $O_{0} B_{0}$. Genauer konstruieren wir $A$. und $B_{c}$. mit Hilfe des Teilungspunkts $O_{1}$ der (ieraden $M S$ hinsichtlich der Ebene $E$ : Machen wir aut $r_{r}^{\infty} J O_{1}=. J O_{0}$ und auf. $S A_{1}=S A_{0}, S B_{1}=S B_{0}$. so gehen $O_{1} A_{1}$ und $O_{1} B_{1}$ bzw. durch $A_{c}$ und $I_{\mathrm{c}}$. Das Bild einer Tangente von $l_{0}$ ist die Tangente ins entsprechenden Punkte vou $k_{c}$ (Art. 72); diese Tangenten schneiden sich aut der Kollineationsachse $e$. Die Ellipsentangenten in $\Lambda_{c}$ und $B_{c}$ sind daher $\| e$; rie

Strecke $A_{c} B_{c}$ ist also ein l'urchmesser von $h_{r}$, und der zu ihm konjugierte Durchmesser $C_{c} D_{c}$ geht durch den Mittelpunkt $X_{c}$ von $A_{c} B_{c} \|$ e. Um die Punkte $C_{c}$ und $D_{c}$ zu finclen, ermitteln wir auf

Fig. 205.

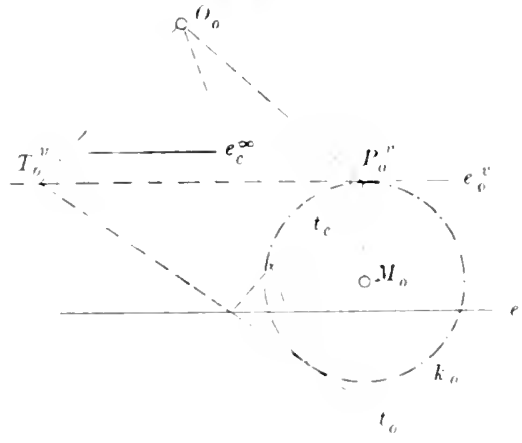
$A_{0} B_{0}$ den Punkt $N_{0}$, am sichersten wieder mittels des Teilungspunktes $O_{1}$, und ziehen durch $N_{0}$ in $l_{0}$ die Sehne $C_{0} D_{0} \| e$, sowie die Geraden $O_{0} C_{0}$ und $O_{0} H_{0}$. Hierdurch ist $l_{c}$ bestimmt. Die Tangenten aus $O_{0}$ an $k_{0}$ beJühren auch $k_{c}{ }^{1}$ ).

219. Zweiter Fall (Fig. 205). Berührt $k_{0}$ die umgelegte Verschwindungslinie $\rho_{0}^{\prime \prime}$ in $P_{0}^{v}$, so ergibt sich als bild von $k$ eine Parabel $l_{i c}$, deren Achse $\| O_{0} I_{0}^{\prime \prime}$ ist. Ziehen wir $O_{0} T_{0}^{v} \perp O_{0} I_{0}^{v}$ bis $e_{0}^{v}$ und vou $T_{0}^{v}$ an $k_{0}$ die Tangente $t_{0}$, so entspricht ih nach Art. 211 eine zu $O_{0} T_{0}^{v}$ parallele Gerade $t_{c}$, die sicl mit $t_{0}$ auf ${ }^{\prime}$

1) Um über $k$ als Grumdkrejs einen geraden Kroiskegel un gregeliener Höhe $l$ zu konstruiıren, errichten wir in $M$ zu $E$ ein $\mathrm{L}$ (nt $. J J=l$ (vgl. Art. 217). Die selurinbaren [mriblinien des Kegrols sind di. Tangrntron alus $P_{c}$ an $k_{c}$. Wir zeichnen sie, olme die Ellipse $l_{c}$ zu henutzen, indern wir $I^{\prime}$ als Bild eines Punkts in $\mathrm{E}$ auffisson, dessen C'mlegung $I_{0}$ wir rrmittelu: $I_{0}$ liegt auf ${ }^{0} P_{c}$, und die Geraden $\boldsymbol{V}_{c} \boldsymbol{P}_{c}$ und.$V_{0} I_{0}$ schueiden sich aut $e$. lann entsprechen den Tangenten aus $I_{0}^{\prime}$ in $l_{0}$ dirs Tangenter ans $I^{\prime}$ an $k^{\circ}$. 
schneidet, und riese ist die Scheiteltangente von $k_{c}$. Dem Berührungspunkte von $t_{0}$ ist der scheitel der Parabel zugeordnet.

220. Dritter Fall (Fig. 206). Schneidet $k_{0}$ die Gerarle $e_{n}^{v}$ in $P_{0}^{v}$ und $Q_{0}^{v}$, so ist $k_{c}$ eine IIyperbel. Den Kreistangenten $p_{0}$ und $y_{0}$ in $P_{0}^{v}$ und $Q_{0}^{v}$ entsprechen die Asymptoten $p_{c} \| O_{0} P_{0}^{v}$ und $q_{c} \| O_{0} Q_{0}^{v}$.

Darstellung der Kugel.

221. Der Kugelmittelpunkt $M$ liege hinter der Ebene $\Pi$ und sei bestimnt lurch seine senkrechte Projektion $M I_{1}$ auf $\Pi$ und seine Entfernung $m$ von dieser Ebene; überdies sei der Riadius $r$ der Kugel gregeben (Fig. 207). Der vom Fig. 206 . Projektionszentrum O ausgehende Tangentenkegel berührt die Kugel in einem Kreise $u$ mnl schneidet $\Pi$ in einem Kegelschnitt $\boldsymbol{u}_{c}$, dem scheinbaren Umrifs der Kugel. Die Brennpunkte von $u_{c}$ sind die lrojektionen der Endpunkte $F$ uud $G$ des anf $H_{+} M_{\mathrm{I}}$ liegenden Kugehlurchmessers (Art. 104).

Legen wir die durch $M M_{1}$ und den Haupt-trahl $O H$ gehende Ebene, die mit der Kugel einen Hauptkreis $F_{i}$ gemein hat, um $H_{3} H_{3}$ in die Bildebene $\mathrm{mm}$, so gelangen $I, F, G$, Fig. 207.

$k, O$ bzw. nach $M_{0}, F_{0}, G_{0}, k_{0}, O_{0}$; rlabei sind $M_{1} M_{0}$ und $H O_{0} \perp H_{\perp} M_{1}$ und bzw. $=m$ und = d. Damn erhalten wir die Brennpunkte

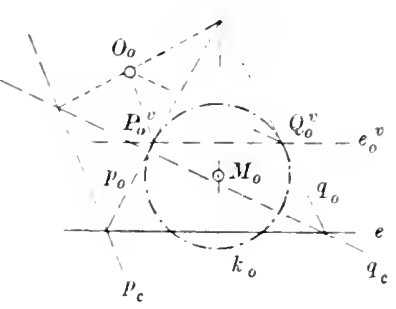
$F_{c}$ und $G_{c}$, sowie die Endpunkte der Hauptachse von $u_{c}$ als die Schnittpunkte von $I I M_{1}$ mit $O_{0} F_{0}$ und $O_{0} G_{0}$, sowie mit den Tangenten aus $O_{0}$ an $k_{0}$.

222. Alle Kugeln, die dem von $O$ an die gegebene Kugel gelegten Tangentenkegel eingeschrieben sind, haben denselben scheinbaren Umrib $u_{c}$. Ist also von der Originalkugel rlas Bild $i_{c}$ des zu $\Pi$ parallelen Hauptkreises $i$ bekannt, so finden wir $u_{*}$ als Cmriß einer Hilfskugel mit dem Hauptkreis $i_{c}$ (Fig. 208). Indem wir die rorhin ausgeführte Koustruktion für diese Hilfskugel wiederholen, verbinden wil den Punkt $H$ mit dem Hittelpunkt $\boldsymbol{M}_{c}$ von $i_{c}$ (d. h. mit dem

Fig. 208 .

$$
{ }^{H}
$$
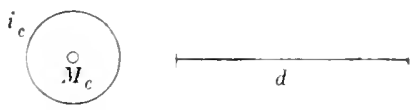

Bilrle des Iittelpunkts $M$ der Originalkugel), ziehen $H O_{0}=d \perp H M_{c}$ und schneiden $H I_{c}$ mit den Geraden von $O_{0}$ nach den Endpunkten des auf $H \boldsymbol{H}_{c}$ senkrechten Durchmessers von $i_{c}$, sowie mit den Tangenten aus $O_{0}$ an $i$.

\section{Verlegung des Projektionszentrums.}

223. Sei $O_{1}$ das ursprüngliche Projektionszentrum mit dem Hauptpunkte $H_{1}$ und der Distanz $d_{1}$, $G$ die spur, $G_{1}^{*}$ der Fluchtpunkt einer Originalgeraden $g$, also $g_{1}=G G_{1}^{\infty}$ ihr 
Bild, ferner $P_{2}$ das Bild eines Punktes $l$ vou !) man soll die Projektionen $g_{2}$ und $I_{2}^{\prime}$ von $g$ und $P$ ans irgend einem anderen Punkte $O_{2}$ ermitteln, der durch seine senkrechte Projektion

Fig. 209.

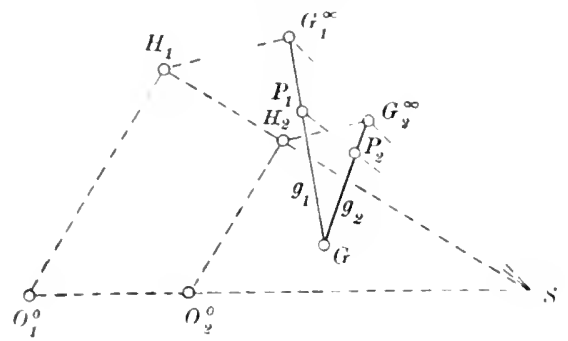

$H_{2}$ anf die Bildebene $\Pi$ und seine Entfernung $d_{2}$ ron dieser gregeben ist (Fig. 209). Wir ermitteln zunächst den Schuittpunkt $S$ der Geraden $O_{1} \theta_{2}$ mit $\Pi$, indem wir die Ebene $O_{1} H_{1} H_{2} O_{2} \mathrm{um}$ $H_{1} H_{2}$ in $\Pi$ umlegen. Der neue Fluchipunkt $G_{2}^{\infty}$ von $g$ liegt auf der Parallele durch $O_{2}$ zu $O_{1} G_{1}^{\infty}$; wir erhalten ihn daher als Schnittpunkt von $G_{1}^{*} S$ mit der Parallele durch $H_{2}$ zu $H_{1} G_{1}^{\infty}$. Iann ist $g_{2}=G^{*} G_{2}^{\infty}$ das gesuchte Bild von g. Der Punkt $I_{2}^{\prime}$ liegt anf der Geraden $S P_{1}^{\prime}$, der Spurlinie der Ebene $O_{1} O_{2} I^{\prime}$.

Liegrt $O_{2}$ auf $O_{1} H_{1}$, so fällt $S$ mit $H_{1}$ zusammen, und der Punkt $G_{2}^{\infty}$ teilt $H_{1} G_{1}^{\infty}$ in demselben Verhältnis wie $O_{2}$ die Strecke $I_{1} O_{1}$.

Ist $O_{1} O_{2} \| \Pi$, so ist $G_{1}^{\infty} G_{2}^{\infty} \# H_{1} H_{2}$ und $P_{1} P_{2} \| H_{1} H_{2}$. Dieser. Fall liegt bei der Konstruktion stereoskopischer Bilder ror.

\section{Angewandte Perspektive.}

Darstellung ebenflächiger Gebilde.

224. Um von allen vertikalen Geraden parallele Bilder zu erhalteu, stellen wir die Bildebene $\Pi$ im folgenden stets vertikal. - Beim Betrachten eines Bildes pflegen wir uns vor seiner Mitte in einer Entfernung aufzustellen, die einerseits genïgend grof ist, $11 \mathrm{~m}$ das Bild als Ganzes ohne Drehmng des Kopfes bequem überblicken zn kömmen, und

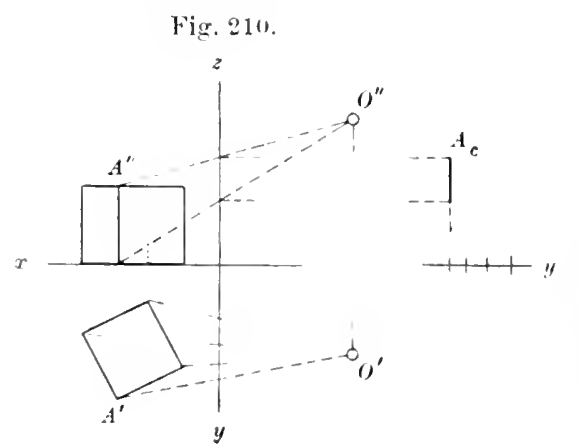
andererseits hinreichend nahe, uın noch die Einzelheiten deutlich wahtzunehmen. Diese Tatsache hat der Zeichner bei der Wabl des Gesichtspunktes $O$ zu beachten. Deshall, wählen wir den Hauptjunkt $H$ nngefähr in der llitte der Bildfläche und die Distanz $d$ in der Regel gleich dem $1_{/ 2}^{1 / 2}$ bis 2 fachen der gröbteu Ausdehnung des Bildes.

Das älteste, aber auch unvollkommenste Verfahren, um die P'erspektive eines Gegenstandes zu konstruiren, ist die sogenannte Glastafel-oder Durchschnittsmethorle (Fig. 210). - Dabei ermittelt man das Bild mit Hilfe von Crund- und Anfifs als den Schnitt der Sehstrahlenpyramide mit der 
Bildebene $\Pi$, die wie eine Seitenribebene $\perp x$ gestellt wird. Dann erbält man auf der $y$-Achse die Grundrisse aller Bildpunkte (die Bildbreiten) und auf der $z$-Achse die Höhen dieser Bildpunkte über rer $y$-Achse. In einer besonderen Figur wird schließlich die Ebene $\Pi \pi$ mit der $y$-Achse und dem entstandenen Bilre - die von $O$ aus sichtbare Seite nach auben — in die Zeichenebene gelegt.

225. Die Spur der horizontalen Bodenebene, auf der die abzubildenden Gegrenstände stehen, heibt Grundlinie; wir bezeichnen sie fortan mit $g$ und die Bodenebene, weil wir sie in der Regel als Grundrißebene benutzen, mit $\Pi_{1}$. - Eine durch $O$ gelegte Horizontalebene (die Horizontebene) schneidet $\Pi$ in einer durch $/ /$ gehenden borizontalen Geraden $h$, dem Horizont des Bildes. Auf $h$ befinden sich die Bilder aller in der Horizontalebene liegenden Punkte. Wird das Bild unter rer Voraussetzung konstruiert, daß der Beschauer auf der Bodenebene steht, so ist der Abstand der Parallelen $g$ und $h$ gleich der Augenhöhe des im Jabstabe der Bildebene gezeichneten Beschauers.

Der Horizont ist die Fluchtlinie aller horizontalen Ebenen und der geometrische Ort der Fluchtpunkte aller horizontalen Geraden. Unter diesen sind für die angewandte Perspektive besonders wichtig:

1. die zu $\Pi$, also zu g parallelen Breitenlinien, mit Bildgeraden. die gleichfalls $\| g$ sind;

2. die zu $\Pi$ senkrechten Tiefenlinien, mit dem Fluchtpunkt $H$ :

3. die Geraden, die mit der Breitenrichtung einen Winkel von $45^{\circ}$ bilden, und die wir im folgenden kurz als $45^{0}$-Linien bezeichnen. Ihre Fluchtpunkte $D_{1}$ und $D_{2}$ liegen auf $h$ im Abstande $d$ vom Hauptpunkt $H$ und heißen die Distanzpunkte des Bildes; dabei ist der links vou $I I$ liegende Punkt $D_{1}$ der Fluchtpunkt der (in der Richtung von vorn nach binten) nach links laufenden $45^{0}$-Linien.

226. Mit Hilfe der Distanzpunkte zeichnet man das Bild eines in der Bodenebene liegendeu Quadratnetzes von gegebener Seitenlänge, dessen Seiten teils die Breiten-, teils die Tiefenrichtung haben, und dessen erste Quadratreihe bis an die Grundlinie reicht (Fig. 211).

Ist die Quadratseite gleich der Längeneinheit, so enthält das abgebildete Netz auf jeder

Fig. 211.

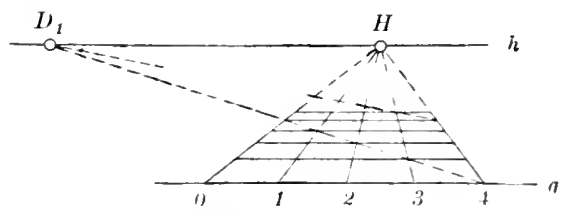
seiner Breiten- und Tiefenlinien einen perspektir gezeichneten II a fotab, und deshalb erweist es sich als ein bequemes Hilfsmittel zum perspektiven Skizzieren. Handelt es sich nämlich zunächst um die Abbildung einer in der Bodenebene liegenden Figur, die in wahrer Gröbe gegeben ist, so überdeckt man sie mit einem ebensolchen Quadratnetz und überträgt ihre Punkte - durch Abschätzen nach dem Angenmaße - in die entsprechenden Maschen des Bildes. Man kann ferner in jedem rler so ermittelten Punkte ein Lot von gegebener Länge zur Bodenebene errichten. Denn das Lot erscheint auch im Bilde als vertikale 
Gerade, und der perspektive Huhenmabstab des Punktes ist gleich seinem Breitenmabstab, weil beide in lerselben Frontebene liegen (rgl. Art. 206 unter b).

227. Grundaufgabe. In der Bodenebene $\Pi_{1}=g, h$ ist hinter der Bildebene $\Pi$ der Punkt $Q$ gegeben durch den Fubpunkt $M$ des von ihm anf $g$ gefällten Lotes und die Länge $m$ der Strecke $M Q$ : den Bildpunkt $Q_{c}$ zu konstruieren (Fig. 212). Man mache auf $g$ entgegengesetzt zur Richtung $I I D_{1}$ rlie strecke $M Q_{1}$ $=m$, so schneidet $D_{1} Q_{1}$ die Gerade $M H$ im gesuchten Punkte $Q_{c}$; denn $D_{2} Q_{2}$ ist das Bild einer $45^{0}$-Linie $u n d Q_{1} I L Q_{c}$ das Bild eines gleichschenklig rechtwinkligen Dreiecks. - Die Punkte $D_{1}, D_{2}$ sind die Teilungspunkte aller Normalenzu $\Pi$ hinsichtlich der durch diese gelegten horizontalen Ebenen (Art. 213).

Lm auf der Bildgeraden $Q_{c} H$ eine gegebene Länge $l$ ron $Q$ c aus nach hinten wiederholt abzutragen, schneiden wir $y$ mit $D_{1} Q_{c}$ in $Q_{1}$, machen auf $g$ entgegengesetzt der Kichtung $H D_{1}$ die

Fig. 212.

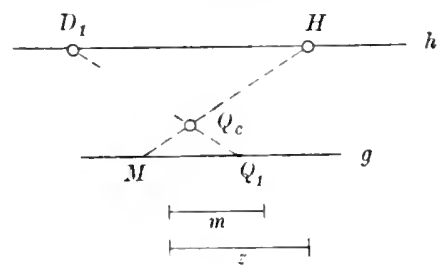

Fig. 213 .

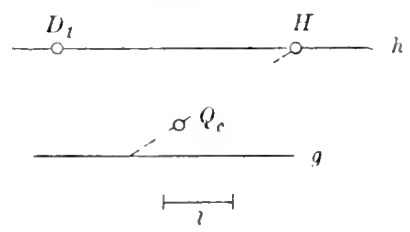

Strecken $Q_{1} R_{1}=R_{1} S_{1} \ldots=1$ und projizieren die Punkte $R_{1}, S_{1} \ldots$ aus $D_{1}$ nach $R_{c}, S_{c} \ldots$ anf die Gerade $Q_{c} I I$ (Fig. 213). - Sind jedoch die Punkte $R_{1}, S_{1} \ldots$ zum Teil oder sämtlich unerreichbar, so tragen wir die Länge 1 rom Spurpunkte $M$ der Geralen $Q_{c} H$ ans entgegrengesetzt zu $H D_{1}$ anf der Grundlinie ab und verbinden den so erhaltenen Punkt $U$ mit $H$. Ziehen wir dann $Q_{c} V_{c} \| g$ bis $U H$, so ist die wahre Länge von $Q V=7$, mithin liegt $R_{c}$ anf der $45^{\circ}$-Linie $D_{1} V_{c}$. Ziehen wir ferner $R_{c} W_{c} \| g$ bis $U H$, so trifft $D_{1} W_{c}$ die Gerade $Q_{c} H$ in $S_{c}$ usw.

Bedentet bei diesen Aufgaben die mit g bezeichnete Gerade nicht die Spur der Bodenebene, sondern das Bild irgend einer in dieser Ebene liegenden Breitenlinie, so gelten dieselben Konstruktionen, sobald der Maßstab der Breitenlinie, d. h. das Bild einer anf ihr liegenden Strecke von gegebener Länge bekannt ist, nur sind dann $m$ und $l$ selbstrer-tandlich in diesem Mabstab anf $g$ abmutragen.

Da die Distanz stets größer ist als die Bildbreite, so befinden sich die Distanzpunte niemals innerhalb des Bildes. Liegen sie? uberhaupt außerhalb der verfügbaren Zeichenfläche, so bedienen wir uns der reduzierten Distanzpunkte, \%. B. des Punktes $\frac{D_{1}}{4}$, der von II um $\frac{d}{4}$ entfernt ist (vgl. Art. 215). Machen wir dann in Fig. 212 auf $g$ die Strecke $M \underset{t}{Q_{1}}={ }_{4}^{m}$. so liegt $Q_{c}$ anf der Geraden $\begin{array}{cc}D_{1} Q_{1} \\ 4 & 4\end{array}$ 
Wir ziehen ferner in Figr. 213 die Gerade $\frac{D_{1}}{4} Q_{c}$ bis $Q_{4}^{Q_{1}}$ und machen anf $g$ die Strecken $\begin{aligned} & Q_{1} \\ & R_{1}\end{aligned}=\begin{aligned} & R_{1} S_{1} \\ & 4\end{aligned}={ }_{4}^{l}$; oder wir machen $M_{4}^{U}={ }_{4}^{l}$ und schneiden die durch $Q_{c}$ gehende Breitenlinie mit $H_{4}^{U}$ in $\frac{V_{c}}{4}$ usw.

228. Grnndaufgabe. Im Punkte $Q$ der Bodenebene $\Pi_{1}$, der durch sein Bild $Q_{c}$ bestimmt ist, ein Lot ( $T^{\prime}$ von der Länge $z$ zu errichten (Fig. 212). Da $Q I^{\prime} \| \Pi$ ist, so ist auch $\hat{Q}_{c} P_{c} \| Q P$, d.h. $\perp g$. Errichten wir im Spurpunkte $M$ von $Q_{c} \|$ zu $g$ das Lot $M N=z$, so liegt $P_{c}^{\prime}$ anf $N H$; dann sind nämlich $Q P$ und $M N$ einander gleich als Parallelen zwischen Parallelen. Oder wir legen durch $Q_{c}$ zwischen $g$ und $h$ eine beliebige Strecke $E F$ und betracliten sie als Bild einer in $\Pi_{1}$ liegenden Geraden mit dem Spurpunkte $E$ und dem Fluchtpunkte $F$. Errichten wir dann $z$ u $g$ das Lot $E Z=z$, so geht $F Z$ durch $P_{c}$ (rgl. hiermit die Aufgabe unter b in Art. 217).

Der Punkt $Q_{c}$ ist der perspektive Grundrif des Punktes $P$ $\left(=P_{c}^{\prime}\right)$. In der angewandten Perspektive bestimmen wir einen Punkt in der Regel durch sein Bild und seinen perspektiven Grundriß - nicht, wie in der theoretischen Perspektive, durch sein Bild nebst Spur- und Eluchtpunkt einer durch ihn gehenden Geraden, oder Spur- und Fluchtlinie einer ihn enthaltenden Ebene.

229. ist die Bildebene parallel zu einer der vertikalen Hauptflächen des dargestellten Gegenstands, so bezeichnet man die Abbildung als Frontansicht oder gerade Ansicht, andernfalls spricht man von einer schrägen Ansicht.

Darstellung einer loppelten Reihe quadratischer Pfeiler iu Frontansicht. In Fig. 214 ist der Hauptpunkt $H$ und der Distanz-

Fig. 214 .

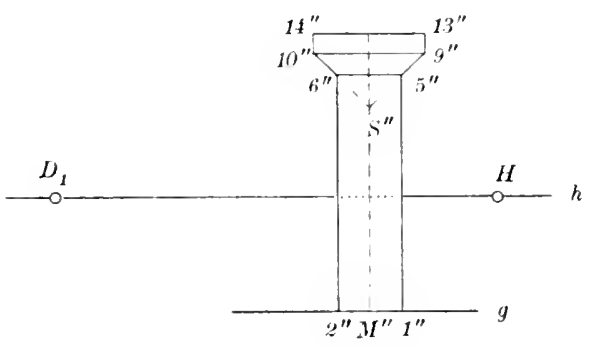

Fig. $214 \mathrm{a}$.

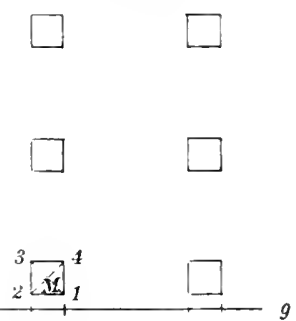

punkt $D_{1}$ anf dem Horizont $h$ gegeben, außerdem die Grundlinie $g$ und vom vordersten Pfeiler auf der linken Seite des Bildes der Aufriß anf lie Zeichenebene $\Pi$. Die Anordnung der sechs in $\Pi_{1}$ liegenden Grundflächen zeigt in verkleinertem Maßstabe Fig. $214 \mathrm{a}$; dabei ist, wenn $a$ die Länge der Kante 12 bezeichnet, der Zwischenraum zwischen zwei nebeneinanderstehenden Pfeilern $=4 a$, zwischen zwei hintereinanderstehenden $=3 a$ und der Abstand des Mittelpunkts $M$ des Quadrats 1234 von der Geraden $g=a$. Hieraus ergibt sich sofort der Aufrif des vordersten der auf der rechten Seite befindlichen Pfeiler. Um eine 
symmetrische Gestaltung des Bildes zn vermojden, haben wir den Hauptpunkt $/ I$ nicht genau in der Mitte zwischen beiden P'feilerreihen gewahlt.

Die Bilder der Grundflächen der drei links stehenden Pfeiler liegen zwischen den Geraden $1^{\prime \prime} H$ und $2^{\prime \prime} H$. Machen wir auf g entgegengesetzt zur Richtung $H D_{1}$ die Strecke $M^{\prime \prime} M_{1}=M^{\prime \prime} M$, d.h. $=a$, so erhalten wir auf $I_{1} M_{1}$ die Bildpunkte $1_{c}, M_{c}, 3_{c}$, und damit die zu $q$ parallelen Seiten $1_{c} 2_{c}$ und $3{ }_{c} 4_{c}$. Ton den dahinter liegenden Quadraten bestimmen wir nach Art. 227 die Bilder ihrer Mittelpunkte. Zu den 'Wecke machen wir anf "/ in der Richtung von $M^{\prime \prime}$ nach $M_{1}$ die Strecke $M^{\prime \prime} U$ gleich der Entfernung zweier anfeinanderfolgenden Mittelpunkte, also $= \pm \boldsymbol{t}$, und ziehen $M_{c} V_{c} \| g$ bis $U M$; dann enthïlt die Gerade $7 V_{1} V_{c}$ das Bild der zu 13 parallelen Diagonale des zweiten Quadrats usw. - Um den perspektiven Grundriß der Deckplatten zu ermittehn, tragen wir die Hälfte der Strecke $9^{\prime \prime} 10^{\prime \prime}$ von $U^{\prime \prime}$ aus auf ! beiderseits ab; (lie Terbindungslinien der so erhaltenen Punkte mit $M$ schneiden z. B. die Diagonalen $1_{c} 3_{c}$ und $2_{c} 4_{c}$ in $9_{c}^{\prime}, 10_{c}^{\prime}$ usw.

Die perspektiven Grundrisse der Pfeiler auf der rechten Seite liegen zwischen denselben Parallelen zu g, wie die entsprechenden 'Teile der links erhaltenen Figur.

Durch die Punkte des perspektiven Grundrisses ziehen wir $\perp g$ die Bilder der vertikalen Kanten der Pfeiler und der Deckplatten nnd begrenzen sie durch die Verbindungslinien der Punkte des Aufrisses mit $H$.

Die vier schrägen Flächen jedes Kapitells bilden eine abgestumpfte Pyramicle, deren Spitze sich auf der vertikalen Mittellinie des betreffenden Pfeilers befindet. Für den vorlersten Pfeiler links ist diese Spitze mit $S$ bezeichnet; dann gehen die Bilder der schrägen Kanten 59 . i 10 ... nach dem Schnittpunkte $S_{c}$ der Vertikalen durch $M I_{c}$ mit $H S^{\prime \prime}$.

Wir können auch leicht die Fluchtpunkte der schrägen Kanten ermitteln. Denn die Kante 5 9 ist die Schnittlinie ihrer ersten und zweiten projizierenden Ebene, folghich ergibt sich ihr Fluclitpunkt $E_{1}$ als Schnittpunkt der Fluchtlinien beider Ebenen. Nun geht die orste projizierende Ebene durch die Gerade 13, die $D_{1}$ zum Fluchtpunkte hat, ihre Fluchtlinie also $\perp h$ durch $D_{1}$. Die zweite projizierende Ebene ist $\perp \Pi$, hat mithin zur Fluchtlinie die Parallele zu $5^{\prime \prime} 9^{\prime \prime}$ durch $I I$. Die Fluchtpunkte $E_{1}, E_{2}, E_{3}, E_{4}$ aller schrägen Kanten liegen also parweise auf den Vertikalen durch $D_{1}$ und $I_{2}$ und auf den Parallelen durch $I I \mathrm{zu}$ den Geraden $5^{\prime \prime} 9^{\prime \prime}$ und $6 " 10^{\prime \prime}$.

230. Darstellung eines Hauses in schräger Ansicht. Das Haus ist in Fig. 215 in Grund- und Aufriß gegreben. Wir wählen den Grundriß $O^{\prime}$ des Auges so, daß clieses zwei Seiten des Hanses sieht, und legen die Bildebene $\Pi$ zweckmäßig durch die vorderste Kante 15, ziehen also die Grundlinie ! durch 1 und zwar angenähert senkrecht zur Halbielungslinie des Winkels $2 O^{\prime} t^{1}$ ).

1) Vgl. Art. 224. Die Geraden $O^{\prime} 2$ mnd $U^{\prime}+$ begrenzen auf o die Bildbreite b. Steht $g$ grenau senkrecht auf der. Halbjurungslinie des Winkels $2 O^{\prime} 4$, sn ergibt sich für die I)istanz, also den Abstand des Punktes ( $)^{\prime}$ von $g$, die für die Jildwitkung günstigste GröBe zwiscben $\frac{3}{2} b$ und $2 b$, wenn jener Winkel ungefahr 7 wixchen $37^{\circ}$ und $28^{\circ}$ liegt. 
Die verlangte P'erspektive soll in Fig. 215 a konstruiert werden; hier ist also die Bildebene $\Pi$ in die Zeichenebene gelegt, in der wir den Horizont $h$ mit dem Hauptpunkte $H$, sowie die Grundlinie $g$ beliebig angenommen haben. Dem Punkte $I I$ entspricht in der anderen Figur der Fubpunkt $H^{\prime}$ des Lotes von $O^{\prime}$ auf $g$; in der Perspektive wird daher die vertikale Strecke 15 in wahrer GröLe so gezeichnet, daß ihr Abstand von $I=I^{\prime} 1$ ist.

Um den perspektiven Grundriß des Hauses zu konstruieren, ermitteln wir zunächst den Fluchtpunkt $F_{1}$ der Seite 14. Zu dem Zwocke ziehen wir in Fig. 215 die Gerade $O^{\prime} F_{1}^{\prime} \| 14$ bis $g$ und übertragen die Strecke $I^{\prime} F_{1}^{\prime}$ von $H$ aus auf den Horizont $h$. Dann ist $1 F_{1}$ das Bild der unbegrenzten Geraden 14. Ebenso könnten wir den Fluchtpunkt $F_{2}$ der Seite 12 bestimmen; ist jedoch Fig. 215 nur eine ungenau gezeichnete Handskizze mit eingeschriebenen Haßen, so konstruieren wir $\mathrm{F}_{2}$ nnmittelbar in Fig. $215 \mathrm{a}$, indem wir die Horizontebene nach oben oder unten in die Zeichenebene umlegen. Kommt hierdurch $O$ nach $O_{0}$, so ist $H O_{0} \perp h$ und gleich der Distanz $O^{\prime} H^{\prime}$, und dann erhalten wir $F_{2}$ mittels $O_{0} F_{2} \perp O_{0} F_{1}$.

Anf der Geraden $1 F_{1}$ haben wir die gleichen Strecken $1 A$ und $A 4$ perspektir abzutragen, und auf der Geraden $1 F_{2}$ die Strecken $1 B, B C, C 2$. Dies geschieht nach der Regel des Teilungspunkts (Art. 213): Machen wir auf $h$ die Strecke $F_{1} T_{1}$ gleich der Länge des Parallelstrahls $F_{1} O$, d. h. $=F_{1} O_{0}$, and $F_{2} T_{2}$ $=F_{2} O_{0}$, so sind $T_{1}$ nnd $T_{2}$ die TeilungsFig. 215.

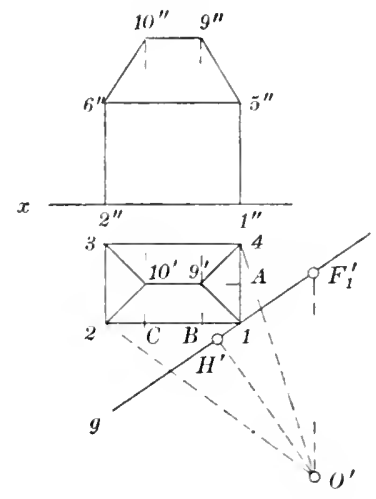

Fig. 215 a.

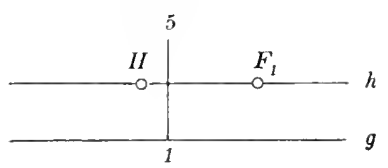
punkte der Geraden $1 F_{1}$ nnd $1 F_{2}$ hinsichtlich der Ebene $\Pi_{1}$. Tragen wir dann die Strecke $1 A$ entgegengesetzt zur Richtung $F_{1} T_{1}$ von 1 aus auf $g$ zweimal ab, so schneiden die Verbindungslinien der so erhaltenen Punkte mit $T_{1}$ die Gerade $1 F_{1}$ in $A_{c}$ und $4_{c}$, und ebenso finden wir auf $1 F_{2}$ mit Hilfe des Teilnngspunkts $T_{2}$ die Punkte $B_{c}, C_{c}, 2_{c}$. Hieraus ergibt sich z. B. $9_{c}^{\prime}$ als Schnittpunkt der Geraden $A_{c} F_{2}$ und $B_{c} F_{1}$.

Die Bilder der Kanten 56 und 58 gehen von 5 nach $F_{2}$ und $F_{1}$.

Uin den First des Daches zu zeichnen, machen wir auf der Geraden 15 die Strecke $1 D$ gleich der gegebenen Firsthöhe und schneiden die Vertikale durch $A_{c}$ mit $D F_{1}$ in $E_{c}$. Dann liegt das Bild des Firsts auf der Geraden $\boldsymbol{E}_{c} \boldsymbol{F}_{2}$; diese trifft die Vertikale durch $9_{c}^{\prime} \mathrm{im}$ Punkte 9 .

Kontrollen: Die Gerade $A_{c} \boldsymbol{E}_{c}$ geht durch den Schnittpunkt der Diagonalen der Seitenfläche $14_{c} 8_{c} 5$; ebenso geht der perspektive Grundrib des Firsts durch den Schnittpunkt von $13_{c}$ und $2_{c} 4_{c}$. - In Fig. 215 halbiert die Gerade $19^{\prime}$ den rechten Winkel 214, mithin liegt ihr Fluchtpunt $F_{3}$ auf der Halbierninglinie des Winkels $F_{1} O_{0} F_{2}$ 
Anmerkung. In derselben Weise, wie hier das bild des Punktes 9 mit Hilfe der strecken $1 A, 1 B, 1 I$ ) ermittelt wurde, findet man auch das Bild eines beliebigen Punktes im Raume, indem man ihn auf die drei zueinauder rechtwinkligen Geraden $14,12,15$ wie auf die Achsen eines Koordinatensystems bezieht und die ibm entsprechenden Achsenabschnitte abbildet.

231. Ist der Abstand $g h$ sehr klein, so wird der perspektive (irundriß, der hei den vorhergehenden Konstruktionen für den weiteren Aufbau des Bildes als unentbehrliche Grundlage dient, nudeutlich und ungenau. Lm dies zu vermeiden, koustruiert man an seiner Stelle den perspektiven Grundrib auf eine genügend weit nach unten (oder nach oben) verschobene Grundribebene (KellergrundriB). Dann liegen entsprechende Punkte der beiden Grundrisse auf rertikalen Geraden, und die Konstruktion des Bildes bleibt völlig unverändert, wenn man die Grundlinie $g$ durch die spur der verschobeneı $\Pi_{1}$ ersetzt und jede Höhe um die Verschiebungsgröße vermehrt.

232. Ist bei der zuletzt behandelten Aufgabe die Distanz d so grob, daß die [mlegung $O_{0}$ des Auges unerreichbar wird, so berlieneu wir uns der in Art. 215 mitgeteilten Methode der reduzierten Punkte: Wir machen auf der Vertikalen durch $H$ die Strecke $I \frac{O_{0}}{n}$ gleich einem passend gewällten Bruchteil ron 1, z. B. $=\frac{d}{n}$, und auf $h$ die Strecke $H \frac{F_{1}}{n}={ }_{n}^{1} H F_{1}:$ dann ist $F_{1} T_{1}=n \cdot \frac{F_{1}}{n} \frac{O_{0}}{n}$. Tragen wir nur die Strecke $\frac{F_{1} O_{0}}{n}{ }_{n}$ von $F_{1}$ aus auf $h$ ab, so erhalten wir den reduzierten 'Teilungspunkt $T_{n}$. Machen wir dann auf $g 1_{n}^{A_{1}}$ gleich dem $n$ ten Teil von $1 A$, so geht die Gerade $\frac{T_{1} A_{1}}{n} \operatorname{durch} A_{c}$.

Jetzt elgibt sich der Fluchtpunkt $F_{2}$ der zu $1 F_{1}$ senkrechten Geraden $1 F_{2}$ mittels ${ }_{n}{ }_{n} F_{2} \perp \frac{O_{0}}{n} F_{1}$ und $H F_{2}=n . M{ }_{n}$.

Ist $F_{2}$ unerreichbar, so erhalten wir die Bildgerade $1 F_{2}$ mit Hilfe les reduzierten Spur]nukts: Wir machen $I I \frac{1}{\|}$ gleich dem ten Teil von $I J 1$ und ziehen durch 1 zur Geraden $\frac{1}{n} F_{2}$ die Parallele $i$. Damn finden wir den 'Teilungspunkt $T_{2}$, indem wir um ${ }_{n}$ einen durch ${ }_{n}$ gehenden Kreisbogen schlagen: schneidet dieser die Gerade $h$ in $Q$, so ist $I I T_{2}=n \cdot I I Q$.

233. Un nach dem unzugänglichen Fluchtpunkte $F_{2}$, der soeben als Schnittpunkt der Gieraden $h$ und $i$ festgelegt worden ist, eine Reihe weiterer Geraden zu ziehen, benutzen wir am einfachsten eine sogenannte 
Fluchtpunktschiene (Fig. 216). I)iese besteht aus drei Linealen, die um eine Achse drehbar sind und sich durch eine Schraube in jeder Lage feststellen lassen. Ton jedem Lineal geht eine Kante, die wir seine Hauptkante nenuen, genau durch die Achse. Das unterste Lineal wollen wir als Zeichenlineal, die beiden andern als Schenkel bezeichnen.

Der Apparat wird in folgender Weise gehandhabt: Wir wählen in der Nähe des dem Punkte $F_{2}$ zugewendeten Randes des Reißbretts ungefähr symmetrisch zu $h$ - zwei Punkte $V$ und 11 , die $h$ und $;$, sowie alle andern Geraden, die noch nach $F_{2}$ gezogen werden sollen. zwischen sich fassen. und befestigen in ihnen senkrecht zur Zeichenfläche zwei Stifte. Dann wird der Apparat so auf die Zeichenfläche gelegt, daß die Hauptkanten $v$ und $w$ der Schenkel die Stifte $V$ und $I V$ ber ühren, und dab das Zeichenlineal mit seiner Hauptkante $u$ an der Geraden $h$ liegt. Wird hierauf die Schraube angezogen und der Apparat so bewegt, daß $v$ und $w$ an $V$ und 11 gleiten, so beschreibt der Drebpunkt $S$ der Lineale einen durch $V$ und $T$ gehenden Kreisbogen, und die Kante $u$ geht beständig durch einen Punkt des Kreises, nämlich seinen Schnittpunkt Fig. 216.

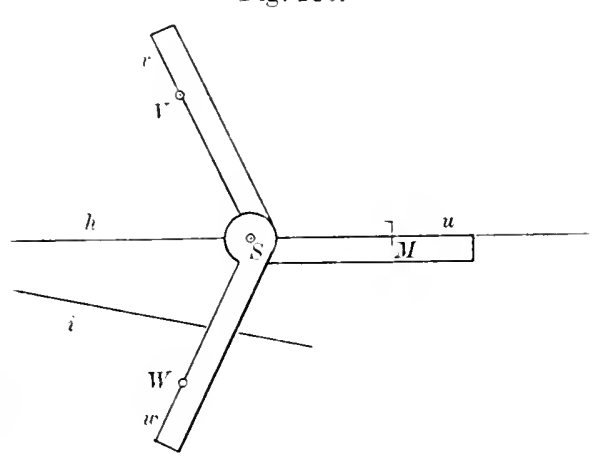
mit $h$. Ist also der Apparat zufällig so eingestellt, daß eine Lage von $u$ mit $i$ zusammenfällt, so treffen sich alle Lagen von $u$ im Schuittpunkte $F_{2}$ von $h$ ind $i$.

Diese Einstellung ist aber durch Probieren leicht zu ermittelu. Dabei ist es zweckmäßig, am Zeichenlineal eine Marke $M$ anzubringen. Nachdem wir den Apparat in der vorhin beschriebenen Lage (vgl. Fig. 216) versuchsweise festgestellt haben, übertragen wir die Marke durch einen Bleistiftstrich 1 auf die Zeichenfläche und lassen daranf die Schenkel so an den Stiften gleiten, daß $u$ sich der Geraden $i$ nähert. Zeigt sich dann, daß die beiden Geraden nicht zur Deckung gebracht werdeu können, daß rielmehr der Fluchtpunkt $F_{2}$ sich links vom festen Schnittpunkt der Kante $u$ mit $h$ befindet, so versehen wir den Strich 1 mit einem nach links weisenden Zeiger. Dann bringen wir den Apparat in seine Anfangslage zurück, lösen die Schraube und verschieben das Zeichenlineal längs der Geraden $h$ ein wenig nach links. Hierauf schrauben wir wieder fest, bezeichnen die neue Lage ron $M$ auf dem Papier durch einen Strich 2 und bewegen den Apparat abermals gegen die Gerade $i$. Liegt jetzt der Fluchtpunkt $F_{2}$ rechts vom neuen Schnittpunkt ron $u$ mit $h$, so bringen wir am Striche 2 einen nach rechts weisenden Zeiger an und suchen die richtige Einstellung von II zwischen 1 und 2 ; andernfalls bekommt 2 einen nach links gerichteten Zeiger, und dann verschieben wir $M$ in dieser Richtung weiter über 2 hinaus usf. 
234. Schräge Ansicht eines im Grund- und Anfrib gegebenen Obelisken (Fig. 217). Hier ist es vorteilhaft, die Bildebene $\Pi$ nicht durch die vorderste Kante des Sockels, sondern durch die Mittellinie $M N$ des Ohelisken zu legen. Wir riehen also, nachdem $\sigma^{\prime}$ passend gewählt ist, die Gerade ! durch.$M$, und zwar so, daß der Grundrib $H^{\prime}$ des Hauptpunkts ungefähr in die Mitte rler Bildbreite fällt. In Fig. 217 a, bei der $\Pi$ in der Zeichenebene liegt, ist $H$ beliebin angenommen und der Abstand $g h$ ungefäh gleich der Augenhöhe eines Jenschen im Mabstab der gegebenen Skizze. Aus dieser entuehmen wir die Gerade $M N$ mit der auf ihr liegenden 'Teilung, sowie den

Fig. 217.

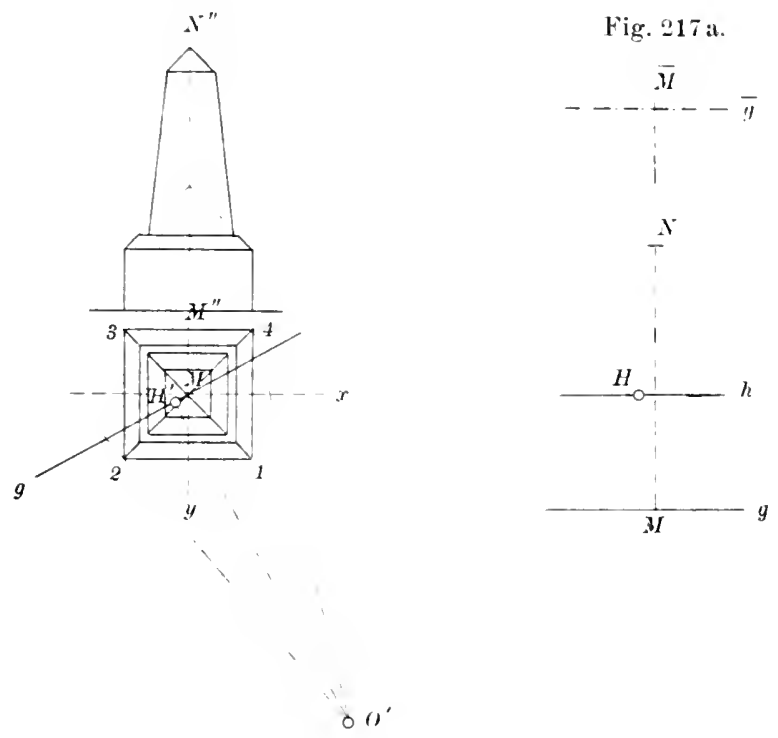

Fluchtpunkt $F_{1}$ der Seite 14 der quadratischen Grundfäche und hestimmen mittels der Emlegung $O_{0}$ vou $O$ die Fluchitpunkte $F_{2}$ und $F_{3}$ der Seite 12 und der Diagronale 13, sowie die Teilungspunkte $T_{1}^{\prime}$ und $T_{2}$ der Geraden 1.1 und 12 . (Vol. Art.230.) Darauf koustrujeren wir den perspektiven Grundrib, am besten auf eine oberhalb $N$ liegende Ebene $\Pi_{1}$ mit der Spur \%. Zu dem Zwecke zeiclinen wir zuerst die Bilder $M F_{1}$ und $M F_{2}$ der durch $M$ gezogenen Geraden $y \| 14$ und $x \mid 12$ und übertragen mittels $T_{1}$ und $T_{2}$ die auf ibnen liegenden $A b-$ schnitte. Darans erhalten wir sofort die Bilder aller Quadrate, aus denen der Grundriß rles Obelisken bestelt; dabei dient rie Diagonale $M F_{3}$ als Kontrolle für die Richtigkeit ler Zeichnung.

Ton jedem der entsprechenden, anf dem Körper liegenden Quadrate kennen wir seinen Mittelpunkt auf der Gerarlen $M N$; wir finden also sein liild aus seinem perspelitiven Grundrib und dem Bild der zu 1:; parallelen Diagonale, oder auch einer zu $x$ orler zu y parallelen Mittellinie. 
235. Ermittelung des Fluchtpunkts einer schrägen, d. h. nicht horizontalen Geraden (Fig. 218 und Skizze 218 a). Der Fluchtpunt $F$ der Geraden a liegt auf der Fluchtlinie $f$ ihrer ersten projizierenden Ebene, d. h. auf der Vertikalen durch den auf

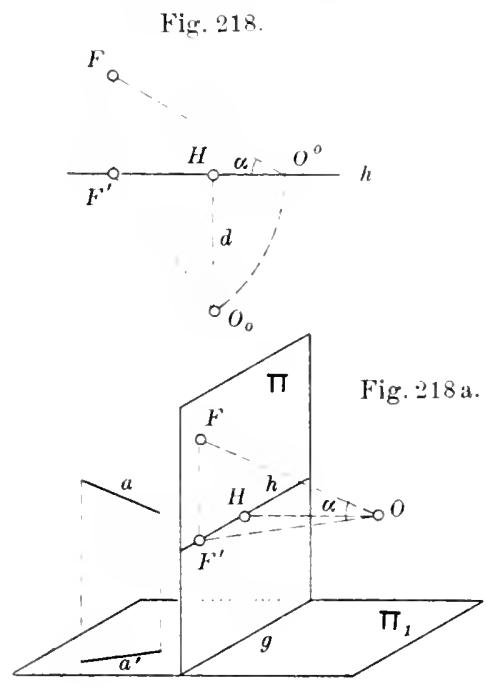

dem Horizont befindlichen Fluchtpunkt $F^{\prime}$ des Grundrisses $a^{\prime}$ von $a$. Kennt man den Neigungswinkel $\alpha$ der Geraden $"$ gegen $\Pi_{1}$, also den Winkel $F O F^{\prime}$, so findet man $F$ durch Umlegung des rechtwinkligen Dreiecks $F O F^{\prime}$ in rie Bildebene $\Pi$. Kommt hierdurch $O$ nach $O^{0}$ auf $h$, so ist $F^{\prime} O^{0}=F^{\prime} O_{0}$, wenn $O_{0}$ wie immer das mit der Horizontebene umgelegte Auge bedeutet; $O^{0}$ ist also der Teilungspunkt der Geraden $a^{\prime}$ hinsichtlich der Ebene $\Pi_{1}$.

Fig. 219.

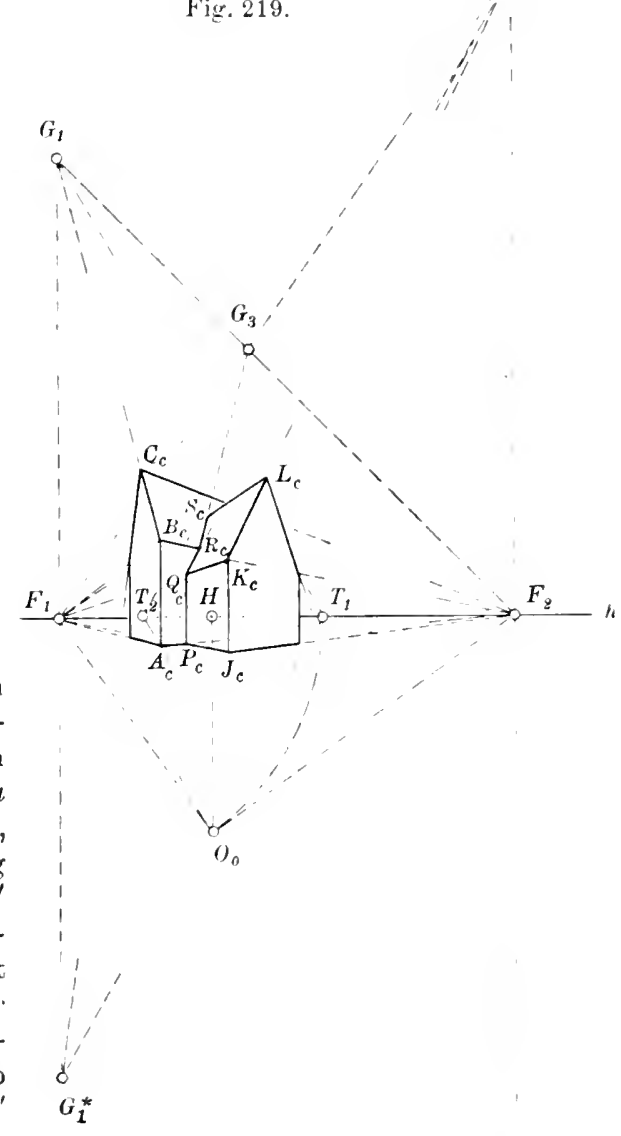

Eine Anwe nd ung zeigt Fig. 2191). In dieser sind $F_{1}$ und $F_{2}$ die Fluchtpunkte der aufeinander senkrechten Grundkanten des dargestellten Gebäudes, $T_{1}$ und $T_{2}$ die zugehörigen Teilungspunkte. Die Horizontalneigung aller Dachflächen soll $60^{\circ}$ betragen. Daraus ergeben sich die Fluchtpunkte $G_{1}, G_{1}^{*}$ und $G_{2}, G_{2}^{*}$ der Giebelkanten, und zwar ist $G_{1} F_{1}=F_{1} G_{1}^{*}$ und $G_{2} F_{2}^{*}=F_{2} G_{2}^{*}$. Die vertikalen Wände $B A P^{\prime}$ und $K J P$ schneiden sich in der vertikalen Geralen $P Q$. Die Schnitt-

1) Nach G. Hauck, Lthrbuch der malerischen Perspektive. 
linie (S $R$ der Dachtläche $L K Q$ mit der Wand $I A I$ ist $\| K L$, geht also im Bilde nach dem Fluchtpunkt $G_{2}$. Lm die Schnittlinie $R S$ der Dachflächen $L K Q$ und $C B R$ zu ermitteln, bestimmen wir ihren Fluchtpunkt $G_{3}$ als Schnittpunkt der Fluchtlinien beider Flächen: Die Geraden $I K$ und $K Q$ haben die Fluchtpunkte $G_{2}$ und $F_{1}$, mithin ist $G_{2} F_{1}$ die Fluchtlinie der Ebene $L K Q$. Ebenso ist $G_{1} F_{2}$ die Fluchtlinie ron $C B R$, mithin $G_{3}=G_{2} F_{1} \times G_{1} F_{2}$.

236. Ermittelung des Hauptpunkts und der Distanz bei einem vorgelegten Bilde. Sind die Fluchtpunkte $F_{1}$ und $F_{2}$ zweier horizontalen, anf einander senkrechten Geraden bekannt, so befindet sich das nach oben umgelegte Auge $O_{0}$ auf dem Halbkreise über $F_{1} F_{2}$ (Fig. 220). Kennt man überdies den Fluchtpunkt $F_{3}$ der Halbierungslinie des ron den Geraden gebildeten Winkels, so halbiert der Strabl $O_{0} F_{3}$ den Winkel $F_{1} O_{0} F_{2}$, geht also durch den Mittelpunkt $M$ des unterhalb $F_{1} F_{2}$ liegenden Halbkreises. Demnach ergibt sich $O_{0}$ als Schnittpunkt des Kreises mit der Geraden $M F_{3}$, und dann ist der

Fig. 220 .

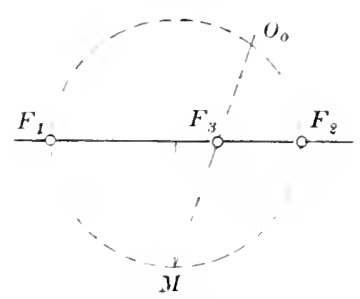

Fig. 221.

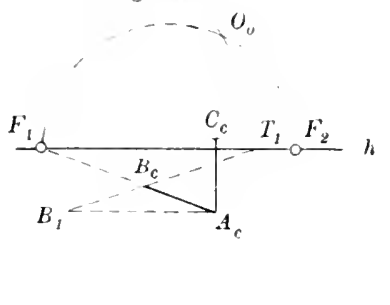

Hauptpunkt $H$ der Fubpunkt des Lotes ron $O_{0}$ auf den Horizont $F_{1} F_{2}$ und die Distanz $=H O_{0}$. - Diese Konstruktion läßt sich z. B. anWenden, wenn das Bild eines horizontalen, schräg liegenden Quadrats bekannt ist.

Fennt man außer $F_{1}$ und $F_{2}$ von einer nach $F_{1}$ gerichteten Bildstrecke $A_{c} B_{c}$ die Länge im Naßstab der durch $A$ gehenden Breitenlinie, weib man z. B., daf $A B$ doppelt so grof ist wie eine durch $A$ gehende Vertikale mit dem Bilde $\boldsymbol{A}_{c} C_{c}$, so mache man auf einer Parallelen zu $F_{1} F_{2}$ die Strecke $A_{c} B_{1}=2 . A_{c} C_{c}$ und schneide $F_{1} F_{2}$ init $B_{1} B_{c}$ in $T_{1}$ (Fig. 221). Dann ist $T_{1}$ der Teilungspunkt von $A B$, und $O_{0}$ liegt auf dem Kreise um $F_{1}$ mit dem Radius $F_{1} T_{1}$.

Hat man außer $F_{1}$ und $F_{2}$ noch die Fluchtpunkte $G_{1}$ und $G_{2}$ zwoier andern horizontalen, anfeinander senkrechten Geraden, so ist $O_{0}$ der Schnittpunkt der Halbkreise über $F_{1} F_{2}$ und $G_{r_{1}} \dot{r}_{2}$. - Bilden die nach $i_{1}$ und $i_{2}$ gehenden Geracten zwar keinen rechten Winkel, woll aber einen Winkel von hekannter Gröle $\varphi$, so ergibt sich als geometrischer Ort für $O_{0}$ der Kieisbogen durch $G_{1}$ und $G_{2}$, der $\varphi$ als Peripheriewinkel faßt.

\section{Schattenkonstruktionen.}

237. Schatten auf die Bodenebene. Der leuchtonde Punkt $L$ sei durch sein bild $I_{c}$ und seinen perspektiven (irundric $L_{c}^{\prime}$ gegeben (Fig. 222). Iann ist der Schatten, den der Punkt $I^{\prime}\left(=I_{c}, I_{c}^{\prime}\right)$ auf 
die Bodenebene $\Pi_{1}$ wirft, der Schnittpunkt $P^{*}$ des ron $L$ nach $I^{\prime}$ gehenden Lichtstrahls mit seinem Grundrib $L^{\prime} P^{\prime}$, also im Bilde $P_{*}^{*}$ $=I_{c} P_{c} \times I_{c}^{\prime} I_{c}^{\prime}$.

Bei Parallelbeleuchtung sind $L_{c}$ und $L_{c}^{\prime}$ die Fluchtpunkte der. Lichtstrahlen und ihrer Grundrisse. Dann liegt $L_{c}^{\prime}$ a uf dem Horizont $h$, ist also stets der Fubpunkt des von $L_{c}$ auf $h$ gefällten Lotes (vgl. Art. 235). $L_{c}$ ist das Bild des Somnemmittelpunkts. Steht dir Sonne vor dem Beschauer, so liegt $L_{c}$ oberhalb $h$ und ist der

Fig. 2.22.

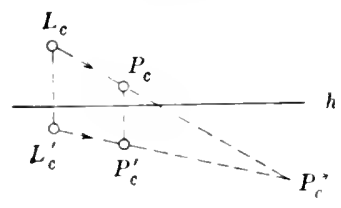

Fig. 223.

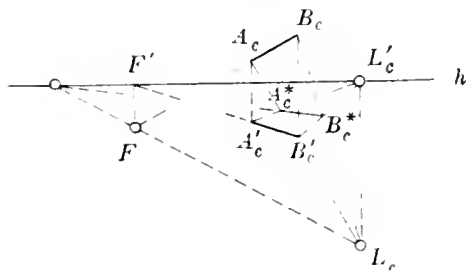

Mittelpunkt des Bildes der Sonne; steht die Sonne hinter dem Beschaver, so liegt $L_{c}$ unterhalb $h$ und ist nur ein virtuelles Bild des Sonnenmittelpunkts. Sind die Lichtstrahlen $\| \Pi$ (Streiflicht), so sind auch ihre Bilder parallel und ihre perspektiven Grundrisse $\| h$.

Der Schatten einer vertikalen Gerarlen auf die Bodenebene $\Pi_{1}$ geht in Bilde nach dem Fluchtpunkte $L_{r}^{\prime}$, der Schatten einer horizontalen Geraden nach dem Fluchtpunkte dieser Geraden (auf $h$ ).

Ist $A_{c} B_{c}$ das Bild einer schrägen (nicht horizontalen) Geraden $A B$, $A_{c}^{\prime} B_{c}^{\prime}$ ihr perspektiver Grundrib, so schneidet die Gerade $A_{c}^{\prime} B_{c}^{\prime}$ den Horizont in ihrem Fluchtpunkte $F^{\prime \prime}$, und der Fluchtpunkt $F$ ron $A B$ ist der Schnittpunkt von $A_{c} B_{c}$ mit der Vertikalen durch $F^{\prime}$ (Fig. 223). Dann ist die Gerade $F I_{c}$ die Fluchtlinie der Lichtstrahlenebene durch $A B$, also ihr Schnittpunkt mit $h$ der Fluchtpunkt des Schattens $A^{*} B^{*}$ ron $A B$ auf $\Pi_{1}$, denn $A^{*} B^{*}$ ist die Schnittlinie jener Lichtstrablenebene mit $\Pi_{1}$.

238. Schatten auf vertikale Ebenen. In Fig. 224 soll der Schatten der Strecke $A B$ auf zwei vertikale Wände $I$ und $I I$ konstruiert werden, die in der Kante $T U$ zusammenstoßen. Dio erste projizierende Ebene des durch $A$ gehenden Lichtstrahls schneidet $I$ in der Tertikalen Fig. 2உ4.

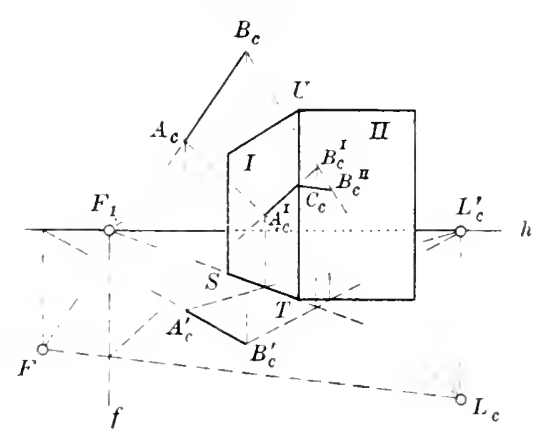
durch den Schnittpunkt der Grundrißspuren beider Ebenen, und diese bestimmt auf dem Lichtstrahl den Schatten $A^{I}$ von $A$ auf $I$. Der ebenso ermittelte Schatten $B^{I}$ des Punktes $B$ liegt auberhalb der begrenzten Fläche $I$; der Schatten von $A B$ auf $I$ reicht also nur bis zum Schnitt- 
punkte $C$ von $A^{I} B^{I}$ mit der liante $T U$. Ist $B^{I I}$ der Schatten von ${ }^{\prime}$ anf die Ebene $I I$, so empfängt diese von $A B$ den Schatten $C^{\prime} B^{I I}$.

Die Fluchtlinio der durch $A B$ gelegten Lichtstrahlenebene verhindet wieder $I_{c}$ mit den Flnchtpunkte $F$ der Geraden $A B$, und die Fluchtinie der Elsene $I$ ist die Vertikale $f$ durch den Fluchtpunkt $F_{1}$ rer in $\Pi_{1}$ liegenden Kante $S T$; der Fluchtpunkt von $A^{I} B^{I}$ ergibt sich daher als Schnittpunkt von $F L_{c}$ mit $f$ : - Die Ebene $I I$ liegt parallel zur Bildebene, hat also eine unendlich ferne Fluchtlinie, mithin ist $C_{c} B_{c}^{I I} \| F L_{i c}$.

Als Anwenlung konstruiere man den Schatten der in Art. 229 largestellten Pfeiler auf die Bodenebene, sowie den Schatten, den einzelne Pfeiler von anderen Pfeilern empfangen.

239. Schatten a f eine beliebige Ebene. Der Schatten des Punktes $P$ auf die Ebene des Dreiecks $A B C$, das durch sein Bild und seinen perspektiven Grundrib bestimmt ist, liegt auf der Schnittlinie der ersten projizierenden Ebene des durch I' gehenden Lichtstrahls mit (ler Ebene $A B C$ und wird ebenso konstruiert, wie früher in senkrechter Projektion (Art. 28).

240. Ermittelung der Eigenschattengrenze eines viereckigen Turms (Fig. 225). Da das Bild der Endfläche $A B C D$ ) des prismatischen Teils des 'Turms von den Geraden $L_{c}^{\prime} A_{c}$ und $L_{c}^{\prime} C_{c}$ gestreift

Fig. 225.

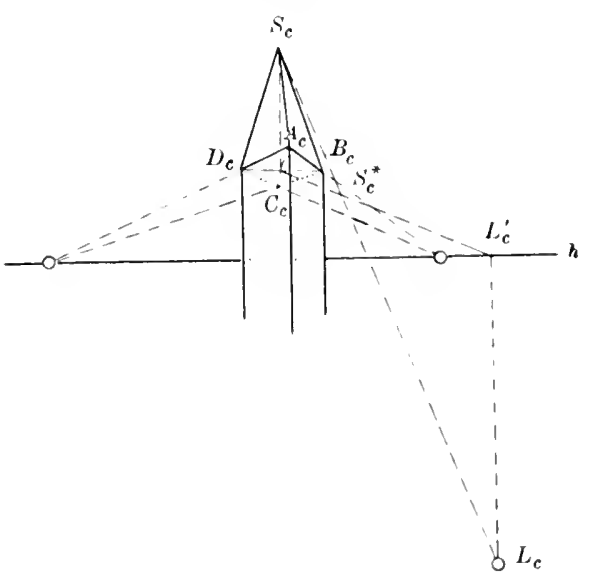

wird, so befinden sich die durch $A B$ und $B C$ gehenden vertikalen Flächen im Schatten. L'm die Eigenschattengrenze des Helms zu ermitteln, konstruieren wir den Schatten $S^{*}$ der Spitze $S$ anf clie Ebene $A B C D$ als Schnittpunkt des durch $S$ gehenden Lichtstrahls mit seiner senkrechten Projektion auf diese Ebene. Dann zeigt sich, dab die Gieraden $S_{c}^{*} B_{c}$ und $S_{c}^{*} C_{c}^{\prime}$ das Viereck streifen, mithin ist von den vier Prramidenflächen nur SBC im Eigenschatten. 


\section{Darstellung krummer Linien und Flächen.}

241. Das Bild einer Kurve wird konstruiert, indem man die Bilder einer genügenden Anzahl von Punkten der Kurve ermittelt und die Bildpunkte aus freier Hand lurch einen stetigen Zng verbindet. Tahei kann man sich auf verhältnismäßig wenige Punkte beschränken, wenn man gleichzeitig ihre Tangenten in die Abbildung ïberträgt.

In Fig. 226 soll die in der horizontalen Ebene " $h$ liegende Kurve $k$, die in Fig. 226a in wahrer Gröbe gegeben ist, perspektiv dargestellt

Fig. 226.

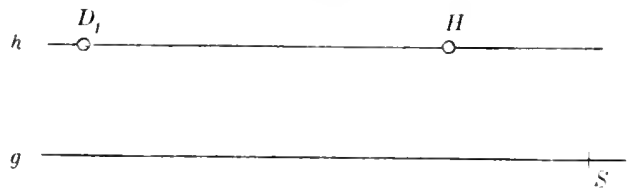

Fig. 225 id.

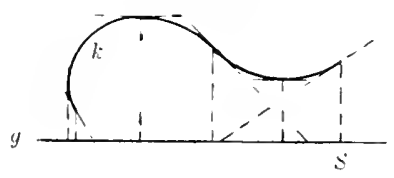

werden, wenn das Projektionszentrum durch den Hauptpunkt $H$ und den Distanzpunkt $U_{1}$ bestimnt ist. Nach (ler in Art. 227 behandelten Grundaufgabe konstruieren wir mit Hilfe von $I I$ und $D_{1}$ vor allem die Bilder der Endpunkte der Kurve, sowie ihres Wendejunkts und der

Fig. 227.

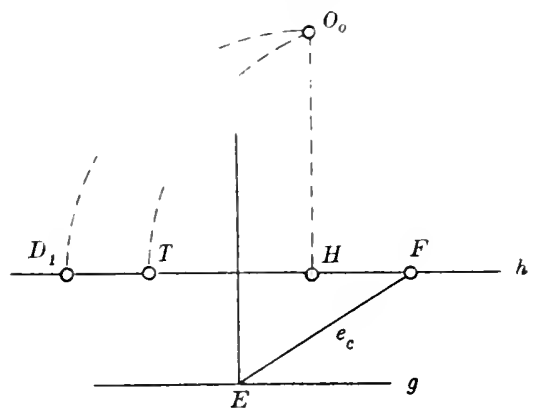

Fig. $227 \mathrm{a}$.

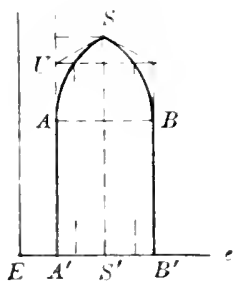

Punkte, deren Tangenten parallel oder senkrecht zu g sind. und gleichzeitig - mittels ihrer Schnittpunkte mit $g$ - auch die Bilder der zugehörigen Tangenten.

Fig. 227 bezieht sich anf die Konstruktion der Perspektive eines Spitzbogens in der vertikalen Ebene $E$, die die Bodenebene $g h$ in der Geraden $e$ schmeidet. Das Projektionsentrum ist wieder durch $I I$ und $D_{1}$ gegeben; die wahre Festalt des Spitzbogens und seine Lage gegen 
die Bildebene zeigt Fig. 227a. Wir ermittehn die Bilder der Punkte A, B. \& mud der zugehörigen 'Tangenten, sowie einiger Zwischenpunkte mit Hilfe der Crmulrisse $A^{\prime}, B^{\prime}, S^{\prime} \ldots$ und ler l'mukte $U$... a uf der Vertikalen $1 A^{\prime}$. Un die auf $e$ liegenden Punkte auf die Bildgerade $e_{c}$ zu übertragen, konstruieren wir in bekannter Woise von $e$ den 'leilungspunkt $T$.

242. Darstellnng (les Kreises (rol. Art. 218). Wir betrachten hier nur noch einmal den praktisch wichtigsten Fall, dab der Krois die Verschwindungsebene weder berührt noch schneidet, so dab er also im Bilde als Ellipse erscheint, und zwar wollen wir zunächst voraussetzen, der Kreis $/$ liege hinter $\Pi$ in einer horizontalen Ebene $\Pi_{1}=g h$; er sei bestimmt durch den Fubpunkt $S$ des Lotes von Mittelpunkt $M I$ a uf $g$, die Länge $m$ von $S M$ und den Radins $r$, außerdem sei auf $h$ rler Hauptpunkt $H$ und der Distanzpunkt $D_{2}$ gegeben (Fig. 22s). Denken wir uns dem Kreise ein Quadrat nugeschrieben, dessen Seiton $\|$ und $\perp g$ sind (Fig. 2.sa), so erhalten wir dessen Bild

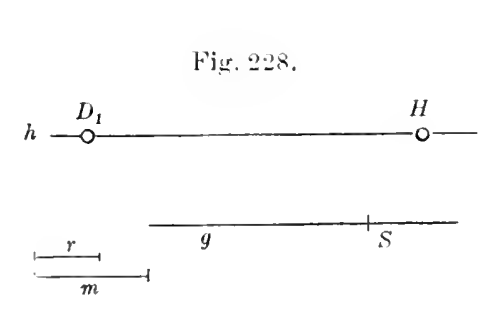

Fig.' 228 a.1

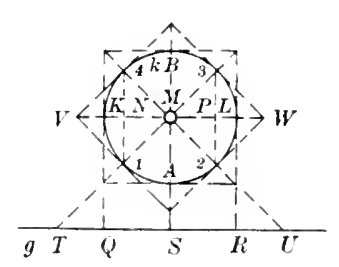

in folgender Weise: Wir machen auf ! $S Q=S R=r, S T=S L^{\circ}=m$; dann gehen die Bilder der auf ! senkrechten Quadratseiten von $Q$ und $l i$ nach $I I$ und die Bilder der Diagonalen von $U$ und $T$ nach den Distanzpunkten $D_{1}$ und $I_{2}$, und die Geraden $I J_{1}$ und $T I_{2}$ treffen sich auf $s / /$ in $M_{i}$. Dadurch sind auch die Bilder der zu q parallelen Seiten und ilhrer Berïhrungspunkte $A$ und $B$, sowie das Bild des zn $g$ prallelen Kreisdurchmessers $K L$, bestimmt; wir kennen also von der Bildellipse $k_{c}$ vier Punkte und ihre Tangenten.

Wir legen ferner um $k$ ein zweites Quadrat, descen Seiten zu den Jiagonalen des ersten parallel sind, und bezeichnen mit $V$ und $W$ die auf $K L$ liegenden Ecken. †ann verhält $\operatorname{sich} M K: M V=1: \sqrt{2}$, mi lat $K I \| \Pi$ ist, so stehen auch die entsprechenden Bildstrecken in demsellsen Verhälnis. Machen wir also auf $K_{c} L_{c}$ die Strecken $\boldsymbol{I}_{c} V_{c}$ und ${ }_{3} \boldsymbol{H}_{c}=\boldsymbol{M}_{c} \boldsymbol{K}_{r} .12$, so gehen die Biller der Seiten des zweiten QuaMats von $r_{c}$ und $W_{c}$ nach den Distangmukten nud bestimmen auf $U W_{1}$ und $T \mathrm{C}_{2}$ rie Berührnngspunkte $1_{c}, 2_{c}, 3,4_{r}$. Oder: Die Geraden 14 nud 29 gehen durch die Mittelpunkte $N$ und $I$ von $M I$ und $M I$.

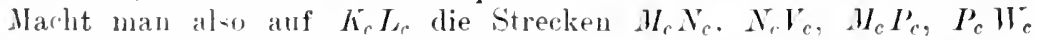
gleich der liathete oines gleichschenklig rechtwinkligen l) reiecks, dessen Hypotenuse $=M_{r} K_{c}$ ist, so bestimmen die Geraden $\Lambda_{c} I I$ und $I_{c}^{\prime} I I$ auf Aen Bildern der liagonalen des ersten Quadrats die Punkte $1_{c}, 2_{c}, 3_{c}$, $t_{c}$ mit den Tangenten $V_{r} 1_{c}$ usw. 
Sind die Distanzpunkte unerreighlar, und ist statt des Punktes $D_{1}$ der reduzierte Distanzpunkt ${ }_{4} J_{1}$ qegeben, so mache

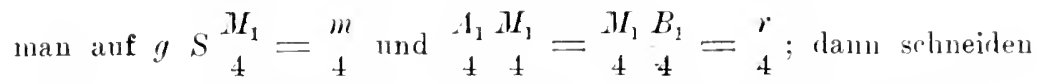

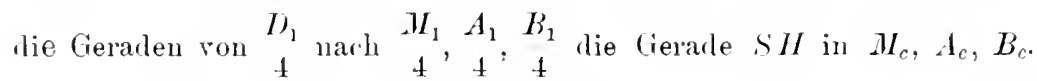
Bedeutet $\begin{aligned} & K \\ & t\end{aligned}$ den Srhnittpunkt ron $\begin{gathered}A_{1} D_{1} \\ 4+\end{gathered}$ mit der Parallelen durch $M_{c}$ zu $g$, so ist $M_{c} K_{c}=M_{c} L_{c}=4 . M_{c}^{K}$. llas Bild res zweiten QuaIrats wird mit Hilfe der Punkte $N_{c}, P_{c}, V_{c}, W_{c}$ ohne Benutzung der Distanzpunkte gefunden.

Anmerkung. Die hier mitgeteilte Konstruktion der Sildellipse aus acht Punkten und ihren Tangenten ist selbstverständlich auch anwendbar, wenn -ich der Kreir, wie in Art.218, in einer beliebigen, durch Spur- und Flnchtlinie bestimmten Ebene befindet, unr sind dann die Punkte $I I$ und $L_{1}$ lureh die früher mit $J$ und $O_{1}$ bezeichneten Punkte zu ersetzen.

243. Ist ron einem horizontal liegenden Kreise von vornherein das Bild $K_{r} L_{c}$ des zu $\Pi$ parallelen Durchmessers gegeben, so findet man die Punkte $A_{c}$ und $B_{c}$, ler Fig. 228 mit Hilfe der Geraden von $K_{c}$ und $L_{c}$ nach $D_{1}$, oder, wenn nur $\frac{D_{1}}{4}$ erreichbar ist, mittels $M_{c}^{K}=M_{t}^{L}=\frac{1}{t} M_{i} K_{c}$ umd durch die Geraden von $\begin{aligned} & D_{1} \text { nach } \\ & t\end{aligned}$ mnd $\frac{L}{4}$. Ist so rlas Bild des ersten umgeschriebenen Quadrats bestimmt, so erhält man das Bild des zweiten genau wie vorhin.

244. Darstellung eines in einer vertikalen Ebene liegenden Kreises (Fig. 229). Die vertikale Ebene $E$ schneirle die Bild-

Figr. 209.

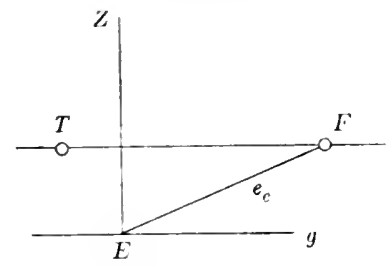

Fig. 229 it.

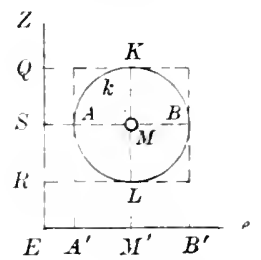

ebene $\Pi$ in $E Z \perp g$, die lorizontale Borlenebene $\Pi_{1}=g h$ in ter Geraden $e\left(e_{c}=E ; F\right)$; ron dieser sei anderdem anf $h$ der Teilungspunkt $T$ bekannt, der wie in Fig. 227 mit Hilfe von $O_{0}$ konstruiert wird. Die Lage des Kreises $k$ in $E$ ist in Fig. 229 a gegeben. Man zeichne zuerst wieder das Bild eines lem Kreise umgeschriebenen Qualrats mit zwei zu E\% parallelen Seiten mittels der Punkte S. Q; I 
und $\boldsymbol{M}^{\prime}, A^{\prime}, B^{\prime}$, mache also auf $g$ die Strecke $E M_{1}^{\prime}=S M$ und $A_{1}^{\prime} M_{1}^{\prime}$ $=M_{1}^{\prime} B_{1}^{\prime}=r$, verbinde $M_{1}^{\prime}, A_{1}^{\prime}, B_{1}^{\prime}$ mit $T$ usw. Dann bestimme man auf dem Bilde des zu $K \%$ parallelen Durchmessers $K I$, wie früher die Punkte $N_{c}^{r}, I_{c}^{\prime}, V_{c}, W_{c}^{*}$ und zeichne das Bild des Quadrats mit der liagonale $\mathrm{l} / \mathrm{l}$.

245. Kennt man von einem in einer beliebigen Ebene liegenden hreise das Bild eines umgeschriebenen Quadrats, von dem zwei Seiten $\| \Pi$ sind, so kam man das Bild des Kreises auch in folgender Weise konstruieren (Fig. 230 und 230a). 'Teilt man die zu $\Pi$ parallelen Seiten und den daranf senkrechten Durchmesser $A B$ in

Fig. 230 .

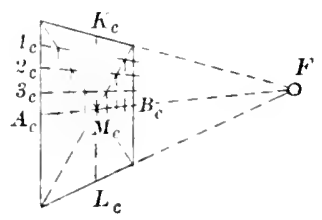

Fig. 230 a.

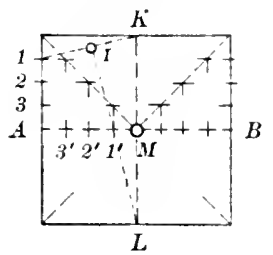

gleich viel gleiche Teile, so schneiden sich z. B. die aufeinander senkjechten Geraden $K l$ und $L 1^{\prime}$ in einem Punkte $I$ des Kreises. Die Teilung $123 \ldots$ auf der zu $\Pi$ parallelen Seite erscheint auch im Bilde als eine Teilung in gleiche Teile; wir übertragen sie durch Geraden nach dem Flnchtpunkte $F$ von $A B$ anf die Diagonalen des Bildtrapezes und von.diesen durch Parallelen zu $K_{c} L_{c}$ auf $A_{c} B_{c}$.

246. Schattenkonstruktion bei einem in schräger Ansicht largestellten Tor (Fig. 231). In rer vertikalen Ebene $E$, die die Bodenebene $\Pi_{1}$ in der Geraden e mit dem Fluchtpunkt $F_{1}$ schneidet, ist über der horizontalen Strecke $A B$ der Halbkreis $l$ gegeben; sein Bild, rie Halbellipse $k_{\text {, }}$, wird nach Art. 244 gefunden. Der Halbkreis ist die Leitkurve eines halben geraden Kreiszylinders, der sich in den durch $A$ und $I$ gehenclen Mantellinien auf zwei vertikale Wände stützt. Diese schneiden tie Bodenebene in zwei auf $e$ senkrechten (ieraden durch die I'unkte $A^{\prime}$ und $I^{\prime}$ mit dem Fluchtpunkt $F_{2}^{\prime}$.

Wir bezeichnen mit $L_{c}$ den Flnchtpunlit der parallelen Lichtstralulen, mit $L_{c}^{\prime}$ deu Fubpunkt des Lotes vou $L_{c}$ anf $h$. Dann int $A_{c}^{\prime} l_{c}^{\prime}$ das Bild des Schattens, den die Bodenebene von der Vertikalen rurch $A^{\prime}$ empfängt. Die Kante $A A^{\prime}$ wirft ferner auf die gegenüberstehende Wand $B B^{\prime} C^{\prime} C$ einen vertikalen Schatten; sein Bild $G_{c} A_{t}^{*}$ seht durch den Schnitpunkt r $_{c}$ von $A_{c}^{\prime} L_{c}^{\prime}$ mit $R_{c}^{\prime} F_{2}^{\prime}$.

Der Shatten, den irgend ein Pnnkt von $k$, z. B. der höchste $K$, auf ten Zylinder wirft, i-t der Schnittpunkt $K^{*}$ des durch $K$ gehenden Lichtstrahls $l$ mit rer /ylinderflache. Wir finclen iln, inden wir durch I parallel zu den Mantellinien des Zylinders eine Ebene $\wedge$ legen und die Mantellinie $i$ konstruieren, in $\operatorname{der} \Lambda$, abgesehen von der dnreh $K$ sehenden Erzensenden, den Zylinder schneidet; dann ist $K^{*}$ der Schnittpunkt vou l mit $i$ (vgl. Art. 130 muter b). Un die Cierade $i$ 
zu ermitteln, suchen wir die Schnittlinie von $\wedge$ mit $E:$ Die Ebene $\wedge$ hat die Fluchtlinie $\mathrm{F}_{2}^{\prime} L_{c}$, und die Fluchtlinie von $\mathrm{E}$ ist die Vertikale durch $F_{1}$; mithin gelit das Bild der Schnittlinie beirler Ebenen vom Schnittpunkte $F_{3}$ ihrer Fluchtlinien nach dem Punkte $K_{c}$. Trifft diese Verbindungslinie die Ellipse $k_{c}$ zum zweiten Male in $J_{c}$, so ist $i_{c}=J_{c} F_{2}$ ind $\boldsymbol{K}_{c}^{*}=l_{c} \times i_{c}$.

Die Tangente aus $F_{3}$ an $k_{r}$ bestimmt die Eigenschattengrenze des Zylinders. - Lie Gerade $F_{3} B_{c}$ schneidet $k_{*}$ im Bilde des Punktes $Q$

Fig. 231 .

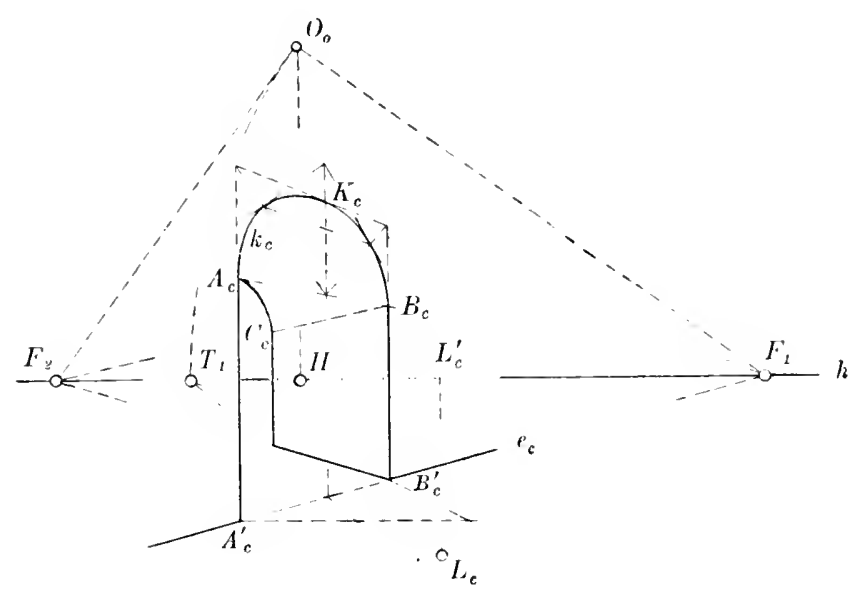

ron $k$, dessen Schatten gerade auf $B C$ fällt. Jeder Punkt von $k$, der zwischen $A$ und $Q$ liegt, wirft seinen Schatten auf die Wand $B B^{\prime} C^{\prime} C$. Dieser Schatten ergibt sich in bekannter Weise aus dem Grundrib des wrich den Punkt gehenden Lichtstrahls.

247. Schattenkonstruktion bei einem aufrecht stehenden geradeu Kreiszylinder mit quadratischer Deckplatte. In Fig. 232 bedeutet $k_{0}$ die Umlegung des in der hinteren Budenebene $\Pi_{1}$ liegenden Grundkreises $k$ des Zylinders. Iie quadratische Platte ist durch ihren umgelegten Grundrif $1_{0}^{\prime} 2_{0}^{\prime} 3_{0}^{\prime} 4_{0}^{\prime}$ und ihren Aufriß auf die Bildebene $\Pi$ gegeben; wie die Figur zeigt, liegt das Rechteck $1265 \| \Pi$. Vir konstruieren zunächst nach Art. 218 oder 242 das Bild $k_{c}$ von $k$ und hierauf in Fig. 232.

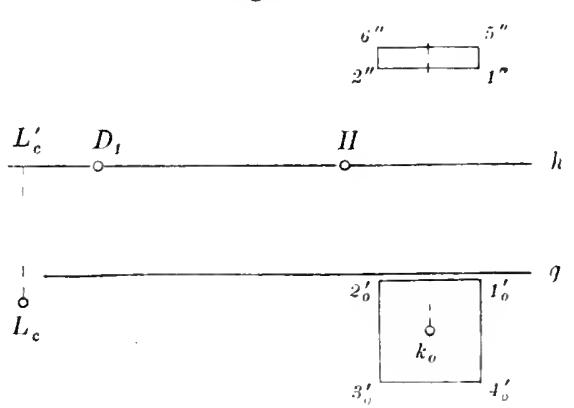
gleicher Weise das Bild $i_{c}$ des wheren Grenzkreises i. Dabei tritt an die Stelle von $g$ die Gerade 1" ${ }^{\prime \prime}$ als Spur der oberen Grenzebene, und entsprechende Punkte 
von $l_{c}$ und $i_{c}$ liegen in Loten z.u $g$. - Die scheinbaren Cmrißlinien $t_{c}$ und $u_{c}$ des Zylinders sind die gemeinsamen vertikalen Tangenten der Ellipsen $k_{c}$ und $i_{c}$; wir erhalten sie am genauesten, ohne $k_{c} z u$ benutzen, auf Grund der perspektiv kollinearen Beziehung zwischen $k_{c}$ und $k_{0}$. Ist $O_{0}$ das umgelegte Projektion:zentrum und der Punkt $O_{0}^{\prime}$ der Geraden $H O_{0}$ sein umgelegter Grundrib, also Abstand $O_{0}^{\prime} g=O_{0} H$ $=H D_{1}$, so geht durch $O_{0}^{\prime}$ die umgelegte Verschwindungslinie von $\Pi_{1}$, und alle Geraden der umgelegten $\Pi_{1}$, die sich in $O_{0}^{\prime}$ als ihrem Terschwindungspunkte schneiden, haben vertikale Bilder. Ziehen wir dahel ans $O_{0}^{\prime}$ an $k_{0}$ die Tangenten $t_{0}$ nnd $u_{0}$, so entsprechen ihnen im Bilde die gesuchten Umriblinien; diese rehen also durch die Schnittpunkte ron $t_{0}$ und $\|_{0}$ mit $\%$

Bezeichnet $I_{c}$ den Fluchtpunkt der Lichtstrahlen, $I_{c}^{\prime}$ seine senkrechte Projektion auf $h$, so wirft jede Mantellinie des Zylinders auf $\Pi_{1}$ einen Schatten, dessen Bild nach $L_{c}^{\prime}$ läuft. Die Grenzlinien dieses Schlagschattens sind also die 'Tangenten $r_{c}$ nnd $u_{c}$ ans $L_{c}^{\prime}$ an $k_{c}$. Wir konstruieren sie genauer mit Hilfe der entsprechenden Tangenten $v_{0}$ und $w_{0}$ an $k_{0}$, die zu $O_{0} L_{c}^{\prime}$ parallel sind: dann geht z. B. $r_{c}$ durch den Schnittpunkt von $v_{0}$ mit $g$. Ist $\Gamma_{0}$ der Berührungspunkt von $v_{0}$ und $k_{0}$, so liefert die Gerade $O_{0} r_{0}$ auf $v_{c}$ den entsprechenden Berïhrungspunkt $V_{c}$ und damit den Anfangspunkt der Eigenschattengrenze des Zylinders.

Um den elliptischen Schlagschatten zu konstruieren, den die Kante 12 der Deckplatte auf clen Zylinder wirft, wählen wir anf 12 eine Reihe ron Punkten $P, Q \ldots$ und ermitteln für die durch sie gehenden Lichtstrahlen die Schnittpunkte $P^{*}, Q^{*}$... mit dem Zylinder: Ziehen wir $P_{c} I_{c}^{\prime} \perp g$ bis $1_{c}^{\prime} 2_{c}^{\prime}$, so ist die Gerade $P_{c}^{\prime} L_{c}^{\prime}$ der perspektive Grundrib des durch $\ddot{P}$ gelegten Lichtstrahls: sie schueidet also $l_{i c}$ in $I_{c}^{* \prime}$, dem perspektiven Grundrib ron $P^{*}$. Genauer erlalten wir $P_{c}^{* \prime}$ mit Hilfe von $k_{0}$ : Wir bestimmen $P_{0}^{\prime}$ als Schnittpunkt ron $1_{0}^{\prime} \varrho_{0}^{\prime}$ mit $O_{0} P_{c}^{\prime}$, ziehen $P_{0}^{\prime} P_{0}^{* \prime} \| O_{0} L_{c}^{\prime}$ bis $l_{0}$ und projizieren den Punkt $P_{c}^{* \prime}$ ans $O_{0}$ anf $P_{c}^{\prime} L_{c}^{\prime}$.

In derselben Weise ergibt sich der Schatten, den der Zylinder von der Kante 14 empfängt.

248. Durchdringung zweier Tonnengewölbe (Fig. 23:3). Tie Wölbllächen sind zwei halbe gerade Kreiszylinder mit horizontalen, -ich rechtwinklig schneidenden Achsen. Der gröbere hat zur Leitkurve den in $\Pi$ liegenden Halbkreis $k$ über dem horizontalen Durchmesser $1 B$, der kleinere den Halbkreis $i$ in der rertikalen Ebene $E$, die $\Pi$ in der Geraden $A A^{\prime}$ rechtwinklig schneidet. Wir geben $i$ durch seine Lmlegung $i_{0}$ in $\Pi$ und bezeichnen mit $E_{0} F_{0}$ die Lmlegung des anf $\Pi$ senkrechten Durchmessers EF. Der von den Wölbflächen überdeckte Raum wird seitlich von vertikalen Ebenen durch die Anfangsmantellinien der Zylinder begrenzt, hinten von einer zu $\Pi$ parallelen Ebene, deren Abstand $F G$ ron $F=A E$ ist, unten von der Borlenebene $\Pi_{1}$ mit der spur $g$. - Das Projektionszentrum $O$ ist durch den Hauptpunkt $H$ und den Distanzpunkt $D_{1}$ gegeben.

Die Gerade $E F$ geht im Bilde ron $A$ nach $I I$, und der Punkt $E_{c}$ liegt auf $E_{0} D_{1}$. Der scheinbare Unriœ des kleineren Zylinders ist die horizontale Tangente $u_{c}$ der nicht gezeichneten Halbellipse $i_{c}$. Nun sind $i_{0}$ und $i_{c}$ perspektiv kollinear mit der spur $A A^{\prime}$ von $E$ als Kollineations- 
achse, der Vertikalen durch $H$ als Fluchtlinie und der Cmlegung $I_{1}$ von $O$ als Kollineationszentrum. 'Tragen wir also auf $h$ die Strecke II $)_{1}$ vou $A A^{\prime}$ ans $a b$, so geht durch den so erhaltenen Punkt $V_{0}$ die umgelegte Verschwindungslinie von $\mathrm{E}$ parallel zur spur $A A^{\prime} ; V_{0}$ ist demnach der V'erschwindungspunkt der Geraden $u_{0}$, die der Geraden $u_{\text {r. }}$ als Lmlegung entspricht, d. h. $u_{0}$ ist die Tangente aus $V_{0}$ an $i_{0}$, und danu geht $u_{c}$ durch den Schnittpunkt von $u_{0}$ mit $A A^{\prime}$.

Cm die Durchdringungskurve zu konstruieren, schneiden wir beide Zylinder durch eine Schar horizontaler Hilfsebenen. Sei s die Spur einer solchen Ehene $\Sigma, T$ ihr Schnittpunkt mit $A A^{\prime}$, so ist $H T$ das Bild der Schnittlinie $t$ ron $\Sigma$ mit E. Die Cierade $s$ trifft $T_{i}$ in den Anfangspunkten $P$ und $Q$ der Mantellinien, die $\Sigma$ aus dem größeren Zylinder schneidet. Ebenso hat $t$ mit $i$ zwei Punkte $h$ und $S$ gemein; ihre Umlegungen $I_{0}$ und $S_{0}$ sind die Schnittpunkte ron $i_{0}$ mit $t_{0}=s$, und die zugehörigen Bildpunkte $R_{e}$ und $S_{e}$ werFig. 233.

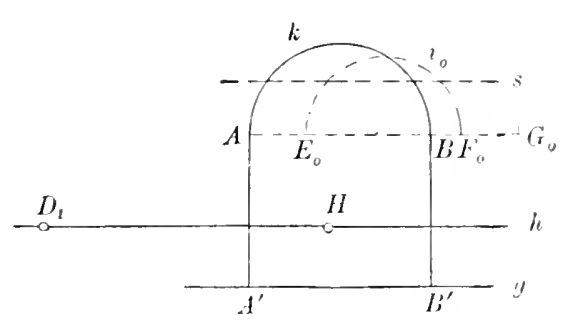
den grefunden, indem wir $R_{0}$ und $S_{0}$ ans $D_{1}$ auf $I T T$ projizieren. Die Horizontalen durch $R_{c}$ und $S_{c}$ sind die Bilder der in $\Sigma$ liegenden Mantellinien des kleineren Zylinders; sie treffen $P \| I$ und $Q H$ in vier Punkten des Bildes der Durchdringungskurve.

Nan bestimme insbesondere die Punkte der Durchdringungskurva auf der höchsten Mlantellinie des kleineren Zylinders, sowie auf seiner Cmriblinie $"$. In $E$ und $F$ hat die Kurve vertikale Tangenten.

249. Frontansicht eines von vier quadratischen Pfeilern getragenen Kreuzwewölbes. Das in Fig. 234 darzustellende Banwerk hat die Gestalt eines freistehenden Triumphbogens mit doppelten l)urcligang; es wird ron vier kongruenten vertikalen Flächen begrenzt, die quadratisch angeordnet sind, und deren vorderste in $\Pi$ liegt. Fig. 23ta zeigt den Schnitt mit der Anfangsebene des Gewölbes. Die Geraden $A E$ und $B F$, sowie $A_{2} B_{2}$ und $E_{2} F_{2}$ sind die Anfangsmantellinien zweier gerader Kreiszylinder: der erste enthält den in $\Pi$ liegenden Halbkreis $k$, der zweite schneiclet die Seitenwände in Halbkreisen mit den Durchmessern $A_{2} E_{2}$ und $B_{2} F_{2}$. Die beiden halben Zylinder durchdringen sich in zwei Halbellipsen - den Gratlinien des Kreuzgewölbes - mit den großen Achsen $A_{3} F_{3}$ und $B_{3} E_{3}$ und der vertikalen kleinen Halbachse $I_{3} N_{3}=\frac{A B}{2}$.

Um die Gratlinien, sowie die in den Seitenwänden liegenden Halbkreise darzustellen, legen wir durch die beiden Halbzylinder eine Schar horizontaler Hilfsebenen. I lie Spur s einer solchen Ebene $\Sigma$ möge $k$ in $P$ und $Q$ und die in $\Pi$ liegenden Eckkanten in $P_{1}$ und $Q_{1}$ treffen. Dann schneidet $\Sigma$ aus dem Bauwerk vier Quadrate $P P_{1} P_{2} P_{\Sigma}, Q Q_{1} Q_{2} Q_{3}$, $R R_{1} R_{2} R_{3}, S S_{1} S_{2} S_{3}$, deren Bilder sofort konstruiert werden können, 
denn die Bilder del auf $\Pi$ senkrechten Seiten gehen nach dem Hauptpunkte $I /$ und die der Diagonalen nach den Distanzpunkten $D_{1}$ und $D_{2}$. Da die Bilder der zu TT parallelen Seiten $\| h$ sind, so ist der eine der beirlen Distanzpunkte bei dieser Konstruktion enthehrlich. Wir erhalten hierdureh von den (iratlinien die P'unkte $I_{3}^{\prime}, S_{3}$ und $Q_{3}, R_{3}$, und von den seitlichen Halbkreisen die Punkte $I_{2}^{\prime}, I_{2}$ und $C_{2}, S_{2}$.

Es empfiehlt sich noch, in den acht gefundenen Punkten die Tangrenten der betreffenden Kurven zu ermitteln. Schneidet die Tangente von $k$ in $P$ die Eekkante $A_{1} A_{1}^{\prime}$ in $T$, so gehen durch denselben Punkt die Tangenten des Halbkreises in $P_{2}$ und der Gratlinie in $P_{3}$. Das

Fig. 234.

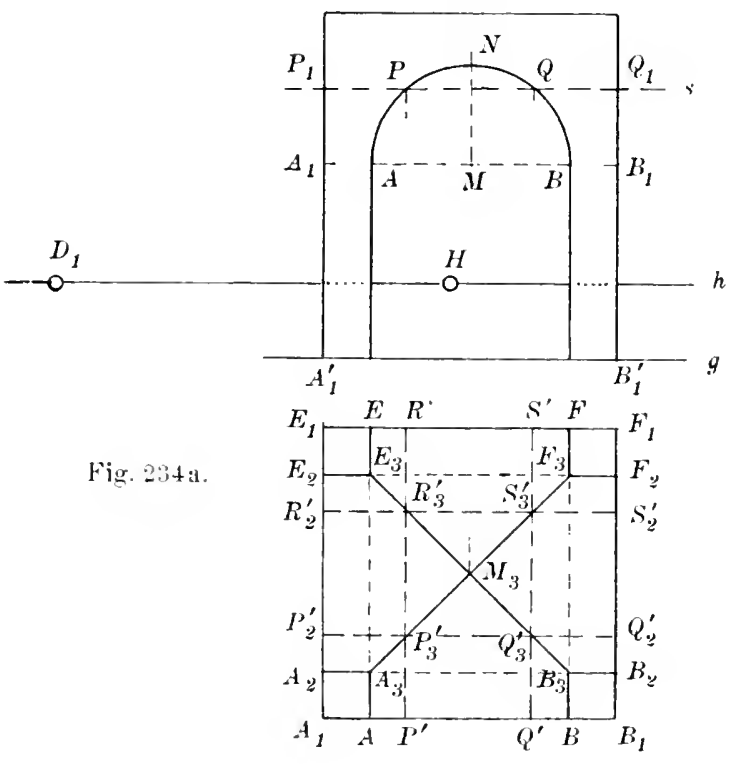

Entsprechende gilt von den Sebnittpunkten rer übrigen Eckkanten mit der durch $T$ gelegten Horizontalebene. - Bezeichnen wir mit $Z$ den Schnittpunkt von $P$ mit der Vertikalen hurch den Mittelpunkt $M$ ron $l_{i}$ und mit $Z_{3}$ den Punkt in gleicher Höhe auf der Vertikalen durch $M_{3}$, so sehneilen sich in $Z_{3}$ die vier Berührungsebenen ler Zylinder in den Erzengenden $P l, Q S, I_{2} Q_{2}, R_{2} S_{2}$; durch $Z_{3}$ gehen also auch die Tangenten der Gratlinien in $I_{3}, Q_{3}, l_{3}, S_{3}$. Am genauesten ergibt sich $Z_{3}$ - wie vorher $Q_{3}$ aus $Q$-, wenn wir $Z Z_{1} \| h$ bis $B_{1} B_{1}^{\prime}$ ziehen und $\zeta_{1}$ mit $I_{1}$ verbinden.

Legen wir eine horizontale Hilfsebene dureh den höchsten Punkt $N$ von $k$ und verfahren in rerselben Weise wie soeben mit dem Punkte $Z$, so erhalten wir auf rer Vertikalen durch $M_{3}$ den Sicheitel $N_{3}$ des Gewolbes, Die Tangenten der Gratlinien in $N_{3}$ gehon im Bilde nach $I_{1}$ und $\mathrm{I}_{2}$.

Wir ermitteln endlich die Punkte der Gratlinien und der Seitenkreise auf ler Cmriblinie $u$ des zu $\Pi$ parallelen Zylinders. 
The Gerale $u$ wird ebenso konstruiert, wie in der vorhergehenden Aufgabe.

In den Punkten $A_{3}, B_{3} \ldots$ werden die gefundenen Kurven ron den vertikalen Pfeilerkanten berührt.

250. Darstellung der Kugel. In Fig. 235 sind $M_{c}$ und $i_{\text {. die }}$ Bilder des Kugrelmittelpunkts $M$ und des zu $\Pi$ parallelen Hauptkreises $i$ : das Projektionszentrum $O$ ist durch $H$ und $D_{1}$ gegeben. Ziehen wir in $i_{c}$ den horizontalen Durchmesser $A_{c} B_{c}$, so bestimmen die Geraden $D_{1} A_{c}$ und $D_{1} B_{c}$ auf $H M I_{c}$ die Bilder $F_{c}$ und $G_{c}$ der Endpunkte des auf $\Pi$ senkrechten Kugeldurchmessers. Nach Art. 104 sind $F_{c}$ und $G_{c}$ die Brenupunkte des Umribkegelschnitts $u_{r}$, wir wollen jedoch diesen Satz, ron dem wir in Art. 221 und 222 bei der Konstruktion des Umrisses ausgingen, gegenwärtig nicht verwenden.

Da die zu $\Pi$ parallelen Kugelkreise in Bild als Kreise erscheinen, so können wir den scheinbaren Umriß der Kugel -- hier die Ellipse

Fig. 235.

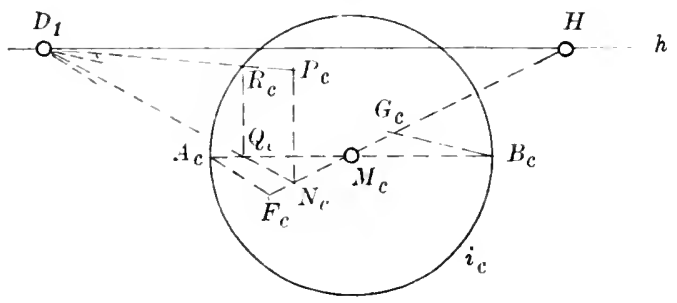

$u_{c}$ - als Einhüllende dieser Bildkreise konstruieren. Lin z. B. den Kreis $k_{c}$ zu zeichnen, der den zwischen $F_{c}$ und $G_{c}$ beliebig gewählten Punkt $N_{c}$ zum Mittelpunkt hat, schneiden wir $A_{c} B_{c}$ mit $D_{1} N_{c}$ in $Q_{c}$ und ziehen $Q_{c} R_{c} \perp A_{c} B_{c}$ bis $i_{c}$, sowie $N_{c} I_{c}^{\prime} \| Q_{c} R_{c}$ bis $D_{1} R_{c}$; dann ist $V_{c} P_{c}$ der Radius von $k_{c}$. Denn $M_{c} N_{c}$ und $M_{c} Q_{c}$ sind die Bilder gleicher Strecken, und dasselbe gilt von $N_{c} P_{c}$ und $Q_{c} R_{c}$; denken wir uns also den Originalkreis $i$ um seinen vertikalen Durchmesser um $90^{\circ}$ gedreht, bis $A$ nach $F$ kommt, so fällt $Q$ auf $N$ und $R$ auf $P$.

Die Ellipse $u_{c}$ berührt den Kreis $i_{c}$ in den Berührungspunkten seiner Tangenten aus $H$.

Um die Eigenschattengrenze der Kugel für Parallelbeleuchtung zu konstruieren, ersetzen wir die gegebene Kugel wie in Art. 222 durch eine Hilfskugel $u m I_{c}$ mit dem Hauptkreise $i_{c}$. Die Eigenschattengrenzen der beiden Kugeln sind parallele, auf der Lichtrichtung senkrechte Hauptkreise, deren Bilder zusammenfallen. Sei $L_{c}$ der Fluchtpunkt der Lichtstrahlen, $E$ die Ebene der Eigenschattengrenze $s$ der Hilfskugel, dann ist die Spur $e$ von $\mathrm{E}$ das Lot von $M_{c}$ auf $I H L_{c}$. Errichten wir ferner in $I I \mathrm{zu} H L_{c}$ das Lot $H O^{0}=H D_{1}$ und ziehen $O^{0} J \perp O^{0} L_{c}$ bis zur Verlängerung von $I I L_{c}$, so geht die Fluchtlinie $e_{c}^{\infty}$ von $\mathrm{E}$ durch den Punkt $J[$ Art. 217, b)]. Legen wir den Kreis $s$ um $e$ in die Zeichenebene um, so deckt er sich mit $i_{c}$; wir können also soin Bild aus dieser Umlegung mittels $e$ und $e_{c}^{\infty}$ konstruieren (Art. 218). 
251. Darstellung einer Umdrehungsfäche mit vertikaler Achse (Fig. 236). Wir betrachten als gegeben die Bilder $a_{c}$ und mo der Achse a der Fläche und der zu $\Pi$ parallelen Meridiankurve m; das Augre $O$ sei wieder durch $I /$ und $D_{1}$ bestimmt. Dann läbt sich der scheinbare ['mrib $u_{c}$ als Einhüllende der Bilder der Parallelkrejser konstruieren: von jedem dieser Kreise kennen wir nämlich das Bild eines zu $\Pi$ parallelen Dnrchmessers, wir erhalten daher das Bild des Kreises

Fi… 236.

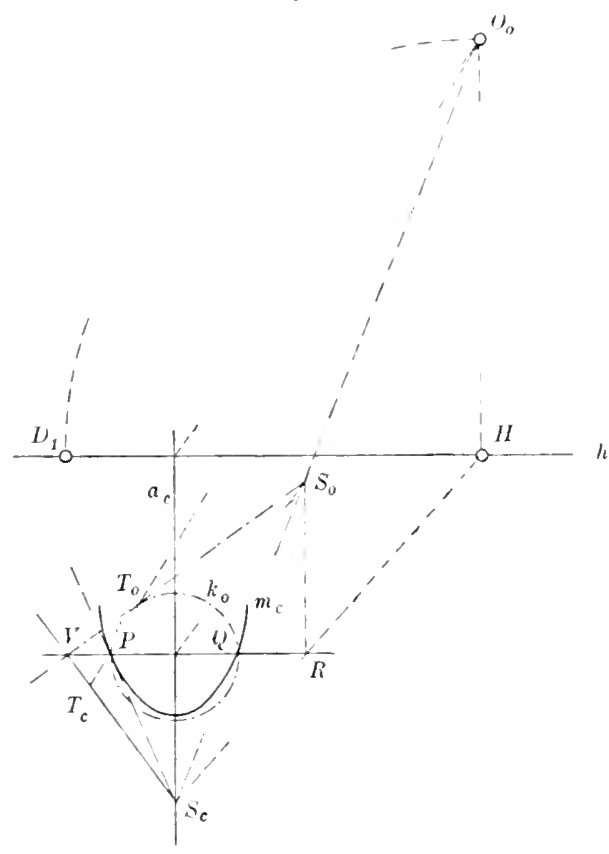

wie in Alt. 243. Kürmer und genaller ist aber das folgende Verfahren, bei dem wir die Parallelkreislerïlsungskegel benntzen. Die Kurve $u_{c}$ ist auch rer scheinbare Cmrib einer andern, zur ersten ähnlichen Emdrehungsflache mit der Achse $a_{c}$ und der. Meridianknre $m_{c}$ : wir diurfen daher bej der Ermittelung von $u_{c}$ die erste Fläche, die lurch $a_{c}$ und $m_{c}$ ïberhaupt noch nicht völlig hestimmt ist, durch die zweite ersetzen (vgl. Art. 222). Wil bezeichnen nun mit li den Parallelkreis dieser zweiten Flache, der die auf $a_{c}$ senkrechte Sehne I'Q ron ma zum Dirchlmesiner hat, mol mit sic die Spitze des zngelıörigen Berïlnungsiegels, also den aut $a_{c}$ liegenten Schnittpunkt der Tangenten von $m_{c}$ in $P$ und (). Lann sind die scheinbaren ['mriblinien des Kegels, d. h. die 'Tangenten aus $S_{c}$ an ras Bild $l_{c}$ von $l_{\text {, }}$ anch Tangenten der Kurve $u_{c}$ in iluren Berïhrungspunkten mit lic (vol. Art. 14t). ['m ans dem Punkte $S_{r}$ an den nicht gezeichneten hegelschnitt $l_{i c}$ Tangenten zn ziehen und ihre Berührungspunkte $T_{c}, C_{c}$ zu bestimmen, beschreiben wir über dem Turchmesier l'(? den Kreis lin

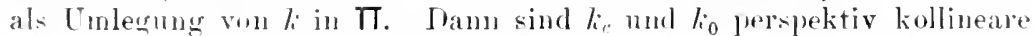
Kurven nit $I^{\prime}\left({ }^{\prime} \text { als Kollineationachse, dem Hol'izont } h=M l\right)_{1}$ als Fluchtlinie und aler [mlegung O) von () als Kollineationsorntrum: dabei ist $/()_{0} \perp H I_{1}$. In dieser kollinearen Beziehung entspricht dem I’unkte $s_{c}$ der Sihnitt mukt $s_{0}$ von $O_{0} S_{c}$ mit der l'arallele zu $a_{c}$ durch Hen shnitpunkt $I i$ von $S^{\prime} I I$ mit $P Q$. dents zu del umgelegten Original-

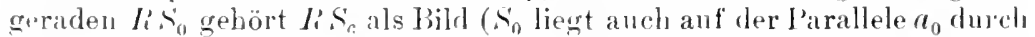
den Mittelpunkt von $P Q$ zur. Terbindungsinie von $O_{0}$ mit dem Schnittpunkt von $a_{c}$ und $h$, denn $a_{0}$ und $a_{c}$ sind sleichfalls entspechente (ierartan). Ziehen wil jetzt aus $S_{0}$ an $k_{0}$ die Taugenten $S_{0} T_{0}$ und

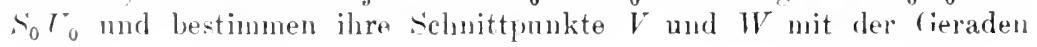


$I^{\prime} Q$, so sind $S_{c} V$ und $S_{c} W$ die gesuchten Tangenten von $l_{c}$, und die entsprechenden Berïhrungspunkte, wie $T_{0}$ und $T_{c}^{\prime}$, liegen auf (ieraden durch $O_{0}$.

\section{Spiogelbilder.}

252. Das Spiegelbild einer Figur in bezug anf die Ebene $\Sigma$ ist bekanntlich dadurch bestimmt, dab seine sämtlichen Punkte zu den entsprechenden Punkten der gegebenen Figur in bezug auf $\Sigma$ symmetrisch liegen. I'm also vom Punkte $P$ sein Spiegelbild $P^{*}$ zu erhalten, fällen wir von $I^{\prime}$ ein Lot anf $\Sigma$, ermitteln seinen Fußpunkt $\mathcal{l}$ und machen anf der Verlängerung von $P Q$ die Strecke $Q P^{*}=I^{\prime}($.

Die perspektive Darstellung dieser Konstruktion gestaltet sich sehr einfach, wenn die Ebene $\Sigma$ horizontal ist (Wasserspiegelung). Dann ist $P^{\prime}(\mathcal{Q}$ vertikal, dasselbe gilt also auch von der Bildgeraden $P_{c} Q_{c}$, und $Q_{c} P_{c}^{*}$ wird $=P_{c} Q_{c}$. Befindet sich in diesem Falle der Punkt $l^{\prime}$ sehr weit hinter der Zeichenebene, so liegt $Q_{c}$ dem Horizont $h$ sehr nahe, mithin fällt $P_{c}^{*}$ mit dem Spiegellild von $P_{c}$ in bezng anf $h$ angenähert zusammen.

253. Spiegelung an einer beliebigen schrägen Ebene. In Fig. 297 ist $h$ der Horizont, $H$ der Hauptpunkt, $O_{0}$ das mit der Horizontebene nach unten umgelegte Auge, $K L M N$ eine rechteckig begrenzte Wand, die die Bodenebene $\Pi_{1}$ in der Geraden $L M$ schneidet, $F_{1}$ der Fluchtpunkt von $L M$ und $K N$. An der Wand soll ein rechteckiger Spiegel $A B C D$ angebracht werden, dessen untere horizontale Kante $A B$ in der Wand liegt, und ressen Ebene $\Sigma$ die Horizontalneigung $\alpha$ bat. Die Bildstrecke $A_{c} B_{c}$ ist nach $F_{1}$ gerichtet. Die schrägen Spiegelkanten $A D$ und $B C$ sind Fallinien von $\Sigma$; ihre senkrechten Projektionen auf die Bodenebene $\Pi_{1}$ sind also $\perp L M$ und gehen daher in Bilde nach dem Fluchtpunkte $F_{2}$, dessen Verbindungslinie mit $O_{0}$ anf $O_{0} F_{1}$ senkrecht steht. Tann liegt der Fluchtpunkt $F_{3}$ von $A D$ und $B C$ anf. der Vertikalen duch $F_{2}$, und zwar bildet die Gerade $O F_{3}^{\prime}$ mit $O F_{2}$ den gegebenen Winkel $\alpha$. Demnach finden wir $F_{3}$, indem wir das rechtwinklige Ireieck $\mathrm{OF}_{2} \mathrm{~F}_{3}$ in die Zeichenebene umlegen: wir machen also auf $h$ rie Strecke $F_{2} O^{0}=F_{2} O_{0}$ und tragen den Winkel $\alpha$ in $O^{0}$ nach unten an $O^{0} F_{2}$ an (vgl. Art. 235). - Die Bildlängen $A_{c} B_{c}$ und $A_{c} D_{c}$ sind beliebig angenommen worden.

Spiegelbild eines Punktes. Tor dem Spiegel ist der Punkt $P$ durch sein Bild $P_{c}$ und seinen perspektiven Grundriß $P_{c}^{\prime}$ gegeben. Cm sein Spiegelbild $P^{*}$ zn konstruieren, ermitteln wir zunächst den Fluchtpunkt $F_{n}$ der Normalen zur Spiegelebene $\Sigma$. Da die Grundrisse dieser Normaleu auf $L M$ senkrecht sind, so haben sio $F_{2}$ zum Fluchtpunkt. mithin liegt $F_{n}$ auf der Vertikalen $F_{2} F_{3}$. Dabei ist $-F_{n} O F_{3}$ ein Rechter; wir erhalten also $F_{n}$, wenn wir als Umlegung dieses Vinkels den Winkel $\mathrm{F}_{n} \mathrm{O}^{0} \mathrm{~F}_{3}$ gleich einem Rechten machen.

Jetzt ist $P_{c} F_{n}$ das Bild des Lotes von $P$ auf $\Sigma$. Wir ermitteln seinen Fußpunkt $Q$, indem wir durch das Lot eine vertikale Hilfsebene legen. Diese schneidet die Bodenebene in einer durch $P^{\prime}$ gehenden Geraden, die auf $L M$ senkrecht steht; ihr Bild ist die Gerade $P_{c}^{\prime} F_{2}$. Lie Hilfsebene schneidet ferner die Wand in der Vertikalen durch den 
Schnittpunkt $I$ von $L, M$ mit der eben gefundenen Geralen und di* spiegelebene $\Sigma$ in einer Fallinie durch den Schnittpunkt si der Vertikalen mit $A B-$ in Bild in der Geraden $S_{c} F_{3}$. Dann trefferi sich $S_{c} F_{3}$ und $I_{c}^{\prime} F_{n}$ im lunkte $Q_{c}$.

Nun ergibt sich endlich das Spiegelbild $P^{*}$ von $I^{\prime}$, wenn wir di* strecke $P$ ' $\mathrm{m}$ sich selbst über Q hinaus verlingern. Zu dem Zwecke ziehen wir durch einen beliebigen Punkt $F_{4}$ von $F_{n} F_{3}$ die Gerade $F_{4}{ }^{\prime}$, bis $Q_{1}$ anf der Verlängerung von $I_{c}^{\prime} P_{c}^{\prime}$ und machen anf dieser Ver-

Fig. :237.

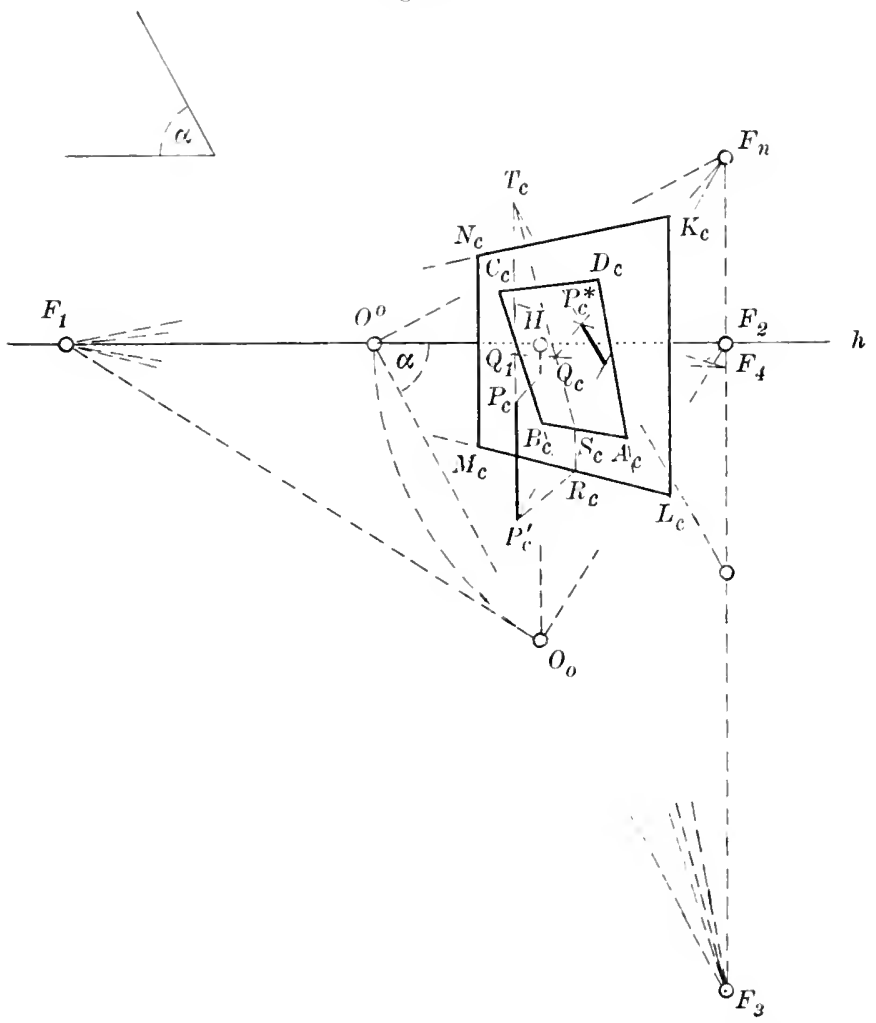

längerung die Strecke $Q_{1} I_{1}^{*}=I^{\prime} Q_{1}$; daun schneidet $F_{4} I_{1}^{*}$ die Gerade $I_{c} F_{n}$ in gesuchten P'unkte $I_{c}^{*}$. Denn von der durch $I^{\prime} Q$ gelegten Hilfsebene ist $F_{n} F_{3}^{\prime}$ die Flnchtinie, $I_{c}^{\prime} P_{c}^{\prime}$ das Bild einer in ihr liegenden Frontlinie, und $F_{4} Q_{1}$ und $F_{4} P_{1}^{*}$ sind die Biller paralleler Graden (vgl. Art. 21t).

Spiegelbild einer Geraden. Der P'unkt $T_{c}=I_{c}^{\prime} I_{c}^{\prime} \times S_{c} Q_{c}$ int das Bild res Schnittpunkts ' $T$ ' der Vertikalen $P I^{\prime \prime}$ mit der Ebene $\Sigma$ : in der Perspektive geht al co das Spiegelbild der trecke $P^{\prime} P^{\prime}$ in seiner Verlängerung durch $T_{c}$. (O)wohl es hierdurch bereits bestimut ist, können wir an Serdem noch seinen Fluchtpmnkt, wie überhaupt den Fluchtpunkt dex Spiegelbilds einfr beliebigen Geraden ermittehn. 
Da parallelen Geralen parallele spiegelbilder entsprechen, so entsprechen sich auch die mendlich fernen Punkte einer (ieraden und ihres Spiegelbilds: dasselbe gilt also in perspektiver Darstellnng von den Fluchtpunkten $\delta$ und $\delta^{*}$ der beiden Geraden. Die Punkte $\delta$ und $i^{*}$ liegen daher in eirer Geraden durch den Normalenfluchtpunkt $F_{n}$ der Spiegelebene $\Sigma$, und zwar wird $\hat{J}^{*}$ ans $\mathfrak{j}$ in derselben Weise gefunden, wie vorhin der Punkt $P_{c}^{*}$ aus dem Punkte $P_{r .}$. Dabei tritt an die Stelle des Punktes $Q_{e}$ del Schnittpunkt 5 . von $\mathbb{F} F_{n}^{\prime}$ mit der Fluchtlinie $f$ von $\Sigma$, weil die der Geraden $\delta F_{n}$ entsprechende Originalgerade im Enendlichen liegt (Fig. 238). Wir finden demnach $F^{*}$, indem wir ฐ․ als eine Bildstrecke betrachten, die $F_{n}$ zum Fluchtpunkte hat, und die wir über : hinaus un sich selbst verlängern sollen. Ziehen wir also durch irgend einen Punkt $E$ der Parallelen durch $F_{n}$ zu $f$ eine Gerade nach $\tilde{F}$, die $f$ in $\mathfrak{F}_{1}$ schneidet, und machen auf $f$ die Strecke $\Sigma . \mathfrak{F}_{1}^{*}=\mathfrak{F}_{1} \Omega$, so trifft $E F_{1}^{*}$ die Gerade $\mathfrak{F} F_{n}$ in gesuchten Fluchtpunkt o*. $^{*}$.

Wenden wir diese Konstruktion anf die vertikale Gerade $P P^{\prime}$ der Fig. 237 an, so ist \& der unendlich ferue Punkt der Geraden, und die Fluchtlinie $f$ von $\Sigma$ ist die Gerache $F_{1} F_{3}$; der Punkt $\Omega$ fallt daher mit $F_{3}$ zusammen. Liegt aber in Fig. 235 der Punkt $\mathfrak{F}$ unendlich fern, so wird $\Omega \widetilde{F}_{1}^{*}=E F_{n}$, und dann ist $\widetilde{F}^{*}$ der Mittelpunkt von $2 \tilde{J}_{n}$. In Fig. 237 ist demmach der Vittelpunkt von $F_{n} F_{3}$ der Fluchtpunkt der Spiegelbilder aller vertikalen Geraden.

Die Konstruktion der Perspektive des Spiegelbilds einer Figur vereinfacht sich beträchtlich, wenn die vertikale Wand, an der der Spiegel hängt, zur Bildebene parallel oder senkrecht steht, odler wenn die Spiegelebene vertikal ist.

\section{Über die ästletische Wirkung der durch Zentralprojektion erhaltewen Bilder.}

254. Der Zentralprojektion wird häufig vorgeworfen, das nach ihren Regeln konstruierte Bild entspreche nicht genau dem Eindruck, den das Auge des Beschauers vom Gegenstande selbst empfängt. In der 'Tat zeigen namentlich die Bilder krummer Flächen, z. B. einer Kngel, zuweilen eine unnatürliche Verzerrung, die um so stärker ist, je weiter das Bild der Fläche vom Hanptpunkte entfernt liegt, und jo kleiner die Distanz ist. Zeichnen wir eine Reihe kongruenter zylindrischer Säulen in Frontansicht, so erscheinen die Sänlen am Rande breiter als die in der Mitte, wie Fig. 239 beweist, die den Schnitt der Horizontebene mit den Säulen und dem Sehstrahlenbindel darstellt.

Diese "Randverzerrungen" sind vom richtigen Gesichtsjunkte O aus selbstverständlich nicht benerkbar, wirken jedoch ̈̈herans störend, wenn wir das Bild mehr yon der Seite ansehen. Sie riihren offenbar daher, daß die projizierenden Strahlen die Bildehene nm so schräger schneiden, je mehr sie rom Hauptstrahl abweichen. Bein natürlichen 
Seheu treten solche Verzerrungen deshalls nicht ein, weil wir dann die einzelnen Säulen überhaupt nicht auf eimmal, sondern schnell nacheinander betrachten, also rewissermaßen jede Sänle für sich auf eine nene, zur jeweiligen blickrichtung senkrechte Bildebene projizieren.

Das perspektive Bild, las die Zentralprojektion liefert, gribt immel den Findruck wieder, ten unser Auge erhalten wïrde, wenn es eine ruhende photographische Kamera wäre. In Wirkliclkeit ist alser nnser Ange eine sehr bewegliche Kamera, die die einzolnen Gegenstände rasch nacheinander fixiert und so eine Fülle von Einzeleindrücken ant-

Fig. 239.

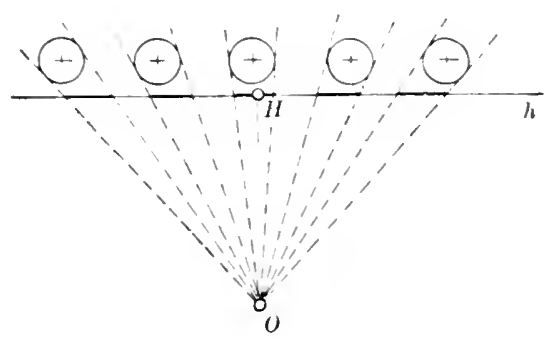

nimmt, die erst nachträglich durch einen geistigen Prozeb zu einem Ganzen, dem subjektiven Anschaun gsbild, verschmolzen werden.

Die gewonnenen Finzeleindritcke in einer Zeichnnng vereinigt wiederzugeben, int mmöglich, da sie einander zum 'Teil widersprechen. So erhalten wir im rorher betrachteten Beispiel ron den am Rande stehenden Siiulen beim direkten Fixieren schmälere und zugleich kleinere Bilder, als von der mittleren säule. Weil jene unter kleinerem Winkel geselsen werden. Ein auf den säulen liegender horizontaler Balken mäbte daher krummlinis gezeichnet werden, was aber ter Forderung widerspräche, dals das Bild jeder Geraden wieder eine Gerade sein soll.

Dem ästhetischen Empfinden des hünstlers bleibt es überlassen, zwischen der strengen perspektiven Formengebung nnd dem aus Einzeleindrücken erhaltenen Anschaunngsbild zu vermitteln; er wird die Randrerzerrungen an:gleichen, indem er im vorigen Beispiel alle Säulen gleich breit darstellt. Am Rande des Pilds befindliche Kugeln dürfen unter lonstimlen einen kreisfömigen Lmrib erhalten, falls sio nicht, wie die halbkugelförmige Kuppel eines Gebändes, in Verbindung mit anderen Bauteilen auftreten. 
Anhang.

\section{Grundzüge der Reliefperspektive.}

255. Um von einem räumlichen Gebilde in gesetzmäßiger Weise eine räumliche Abbildung herzustellen, wählen wir im Raume eineu Punkt $O$ und zwei parallele (vertikale) Ebenen $\Pi$ und $\Pi_{1}^{x}$ und treffen die folgenden Festsetzungen:

1. Die Verbindungslinien entsprechender Punkte in Original- und Bildfigur sollen durch 0 gehen.

2. Jeder Punkt der Ebene $\Pi$ soll sich selbst entsprechen.

3. Das Bild jedes unendlich fernen Punktes soll sich in der Ebeue $\Pi_{1}^{\infty}$ befinden.

4. Das Bild jeder geraden Linie soll wieder eine Gerade sein.

Dann ist zu jeder Originalgeraden $g$ die Bildgerade $g_{1}$ eindeutig bestimmt, denn diese geht durch den Schnittpunkt $G^{\prime}$ von $g$ mit $\Pi$ and

Fig. 240.

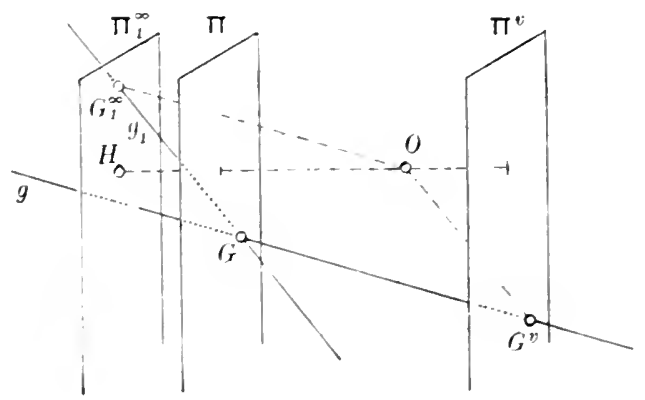

durch das Bild des unendlich fernen Punktes von $y$, d. h. den Schnittpunkt $G_{1}^{\infty}$ von $\Pi_{1}^{\infty}$ init dem Parallelstrahl durch 0 zu g (Fig. 240). Das Bild $F_{1}$ eines beliebigen Raumpunktes $I^{\prime}$ ergibt sich als Schnittpunkt des Strahls $O P$ mit dem Bilde irgend einer durch $P^{\prime}$ gehenden Geraden.

Das auf solche Weise konstruierte Bild einer Raumfigur wird das Relief der Figur genannt. Beide Figuren heißen im allgemeinsten Sinne perspektiv kollinear. Der Punkt $O$ wird als Kollineationszentrum (Gesichtspunkt), die Ebene $\Pi$ als Kollineationsebene, 
die Ebene $\Pi_{1}^{\infty}$ als Fluchtohene bezeichnet. Wir nennen ferner $C_{x}$ den Spurpunkt, $f_{1}^{\infty}$ den Fluchtpunkt der Geraden $g$ und ver-tehen unter Hauptfluchtpunkt den Fubpunkt $I$ des Lotes von O auf $\Pi_{1}^{x}$, also den Fluchtpunkt aller Normalen zu $\Pi$.

Dureh nuser Abbildungserfahren wird der nnendliche Originallaum, der von $O$ aus gesehen sich hinter der Ebene $T$ befindet, in einem Raume von begrenzter Tiefenauslehnung - zwischen $\Pi$ und $\Pi_{1}^{\infty}$ - abgebildet. Die Entfernung der Ebenen $\Pi$ und $\Pi_{1}^{x}$ heißt die Tiefe des Reliefs. Wird diese gleich Null, so verwandelt sich das Relief in eine gewönnliche Zentralprojektion anf die Ebene $\Pi$.

256. Dem nuendlich fernen Punkte der Billgeraden $g_{1}$ entspricht als Originalpunkt der Schnittpunkt ( ${ }^{v}$ vou !) mit der Parallele durch () zu $g_{1}$; wir bezeichnen ihn wieder als den Verschwindnngspunkt von $g$. Wegen $G^{v} G_{r}=O G_{r}^{\infty}$ ist auch Abstand $G^{v} \Pi=$ Abstand $O \Pi_{1}^{\infty}$, l. h. konstant für alle Originalgeraken. Demnach liegen die Verschwindungspunkte aller Geraden in einer zu M parallelen Ebene, der Verschwindnugsebene $\Pi^{v}$, und es ist Abstand $\Pi^{v} \Pi$ $=$ Abstand $O \Pi_{1}^{\infty}$.

257. Das Relief $E_{1}$ einer Ebene $E$ ist wieder eine Ebene, deun allen Geraden in $E$ entsprechen Bildgeraden, die einander sämtlich schneiden. Bestimmen wir von $\mathbf{E}$ die Spurlinie $e=\Pi \times \mathbf{E}$, sowie die Fluchtlinie $e_{1}^{\infty}$, d. L. die Schnittlinie von $\Pi_{1}^{\infty}$ mit der Parallel-

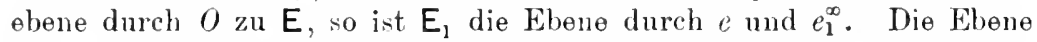
E schneidet $\Pi^{v}$ in der Verschwindungslinie $e^{v}$, deren Verbindungsebene mit $O \| E_{\text {, }}$ ist.

Entsprechende Figuren in $E_{\text {nnd }} E_{1}$ sind perspektiv kollinear mit $O$ als Zentrum, $e$ als Achse und $e_{1}^{\infty}$ als Fluchtlinie. - Ist $E \| \Pi$, so gilt dasselbe von $E_{1}$, und entsprechende Figuren beider Ebenen sind einander ähnlich.

Die Fluchtlinie aller horizontalen Ebenen geht durch $I I$ und heißt der Horizont des Reliefs.

Um das Relief eines Gegenstandes zu konstruieren, bedienen wir uns des Grund-und Anfrißverfahrens; dabei stellen wir die Ebenen $\Pi$ und $\Pi_{1}^{\infty}$ senkrecht zur Projektionsachse $x$.

258. Abbilrlung der Kugel. Da jeder ebene Schnitt der Kugel $K$ in einen Kegelschnitt übergeht, so erhalten wir als Relief $K_{1}$ eine Fläche zweiter Ordnung ohne gerade Linien, und zwar ein Ellipsoid, elliptisches Paraboloid oder zweischaliges Hyperboloid, je nachden die Kugrel mit der Verschwindungsebene $\Pi^{v}$ keinen Punkt gemein hat, orler sie in einem Punkte berührt, orler in einem Kreise schneidet. Insbesondere entsteht eine Lmdrehungsfläche zweiter ()rdnung, wenn der Kugelmittelpunkt anf der Geraden OH lierrt.

Der Tangentenkegrel an $\mathrm{K}_{1}$ ans irgend einem l'unkte $L_{1}$ ist das Relief des Tangentenkegels aus dem entsprechenden Punkte $L$, an $\mathrm{K}$, und da lieser Kegel die Kngel in einem Kreise s berührt, so folgt: Die Eigenschattengrenze $s_{1}$ der Fläche zweiter Ordnung $K_{1}$ ist ein Kegelschnitt. - Ist $L_{1}$ mentlich fern und $\mathrm{K}_{1}$ ein elliptisches Paraboloirl, so liegt $I$ in der Ebene $\Pi^{v}$, die in diesem Falle von $\mathrm{K}$ in 
einem Punkte $P$ berührt wird. Dann beriuhrt auch der Kreis s die Ebene $\Pi^{\prime}$ in $P$, mithin ist die Eigenschattengrenzes, des elliptischen Paraboloids bei Parallelbeleuchtung eine Parabel.

259. Sonderfälle der Reliefperspektive. a) Ist $O$ unendlich fern, so entspricht jedem unendlich fermen Originalpunkte ein unendlich fernes Bild; die Bilder paralleler Geraden sind also parallel, und die Ebenen $\Pi_{1}^{\infty}$ und $\Pi^{v}$ fallen mit der unendlich fernen Ebene des Raumes zusammen. Wir bezeichnen die Beziehung zwischen Original- nurl Bildfigur in diesem Falle als (räumliche) Affinität in perspektiver Lage.

b) Liegt die Ebene $\Pi$ unendlich fern, so entspricht jeder Originalseraden eine parallele Bildgerade und entsprechende Strecken stehen in konstantem Verhältnis. Die Ebenen $\Pi_{1}^{x}$ und $\Pi^{v}$ fallen mit der unendlich fernen $\Pi$ zusammen: $\ddot{A} h n l i c h k e i t$ in perspektiver Lage.

c) Ist sowohl $O$ als auch $\Pi$ unendlich ferm, so sind Original- und Bildfigur kongruent und parallel. 



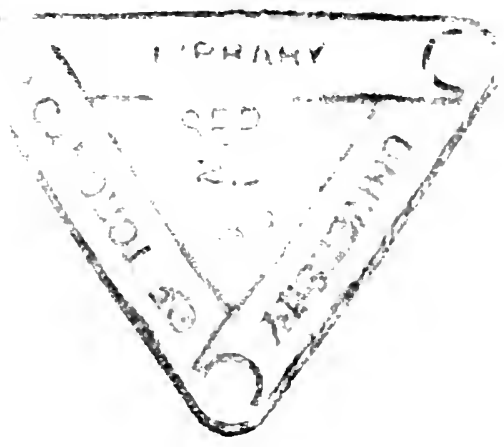


